\title{
The Design, Fabrication, and Commissioning of a High-Speed Aeroacoustic Wind Tunnel for Studies of Surface Pressure Fluctuations Beneath Turbulent Boundary Layers
}

\author{
by \\ Frank Giardino, B.Eng. \\ A thesis submitted to the \\ Faculty of Graduate and Postdoctoral Affairs \\ in partial fulfillment of the requirements for the degree of
}

Master's of Applied Science in Aerospace Engineering

Ottawa-Carleton Institute for Mechanical and Aerospace Engineering

Department of Mechanical and Aerospace Engineering

Carleton University

Ottawa, Ontario

May, 2018 
C) Copyright

Frank Giardino, 2018 
The undersigned hereby recommends to the

Faculty of Graduate and Postdoctoral Affairs acceptance of the thesis

\section{The Design, Fabrication, and Commissioning of a High-Speed Aeroacoustic Wind Tunnel for Studies of Surface Pressure Fluctuations Beneath Turbulent Boundary Layers}

submitted by Frank Giardino, B.Eng.

in partial fulfillment of the requirements for the degree of

Master's of Applied Science in Aerospace Engineering

Professor Joana Rocha, Thesis Supervisor

Professor Ronald E. Miller, Chair, Department of Mechanical and Aerospace Engineering

Ottawa-Carleton Institute for Mechanical and Aerospace Engineering

Department of Mechanical and Aerospace Engineering

Carleton University

May, 2018 


\section{Abstract}

The High-Speed Aeroacoustic Wind Tunnel (HSAWT) at Carleton University is a newly commissioned facility with the purpose of facilitating experimental studies of Turbulent Boundary Layer (TBL) induced surface pressure fluctuations. This research is intended for applications regarding aircraft noise generation from structures exposed to high-speed flow. This open-jet, blowdown facility is unique in Canada and one of a few aeroacoustic wind tunnels in the world capable of achieving speeds up to Mach 0.8 .

The details of the complete design and fabrication methodology for all wind tunnel duct components, control system hardware, and instrumentation systems is discussed; along with the numerical simulations performed in the validation of the designed components. Preliminary experimental flow measurements and characterization of the wind tunnel control system is examined. The results of initial measurements of TBL surface pressure fluctuations developed over a flat test section plate are compared with established data and empirical models in literature. 


\section{Acknowledgements}

First and foremost, I would like to thank my thesis and research supervisor, Professor Joana Rocha, for her support and invaluable guidance provided throughout my entire Master's Degree program. Our countless free and open discussions during research meetings has helped me to develop a better understanding and appreciation regarding the complex topics of fluid mechanics and aeroacoustics. I have no doubt that my experiences the past couple of years as a part of her research team will benefit me in my future career initiatives and endeavors.

I would like to express my sincere gratitude to Alex Proctor, Kevin Sangster, Steve Truttman, and Ahmad Rafiei for their technical support and assistance in the design, fabrication, and installation of some of the major research components in the wind tunnel facility. Without their help in taking the time out of their busy schedules, none of this research would be possible.

I also would like thank my fellow research colleagues and friends, Jared VanBlitterswyk, Steven Sonnenburg, Gerry Desmarais, and Mitch Kibsey. Large accomplishments never take the effort of just one person and I am so grateful for their continuous encouragement, suggestions, and ideas in our discussions involving my research.

I wish to extend my thanks to Carleton University, the Natural Sciences and Engineering Research Council (NSERC), and the Consortium for Research and Innovation in Aerospace in Quebec (CRIAQ) for their generous financial support of my work. 
Finally, I wish to acknowledge the tremendous love and support that I have received from both my family and Tanya McNally. I would not be the man I am today, let alone be in a position to obtain a Master's Degree, without their direction and relentless support. Thanks for pushing me to pursue my dreams and to strive to be the best person I can be. Tanya, you are my best friend and I am so glad that you have been right there by my side the entire way. 


\section{Table of Contents}

Abstract iv

Acknowledgements $\quad$ v

Table of Contents vii

List of Figures $\quad$ xii

List of Tables $\quad$ xviii

Nomenclature xix

\begin{tabular}{|ll}
\hline 1 Introduction & 1
\end{tabular}

1.1 Motivation . . . . . . . . . . . . . . . . . . . . 2

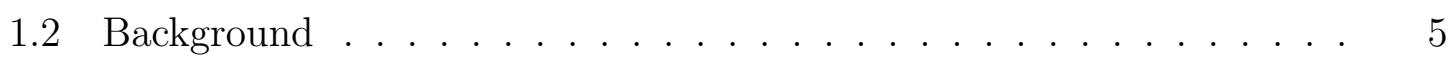

1.2.1 Review of TBL Surface Pressure Fluctuation Studies . . . . 6

1.2.2 Aeroacoustic Wind Tunnel Design and Survey of Existing Facilities . . . . . . . . . . . . . . . . 15

$1.2 .3 \quad$ Existing Carleton High-Speed Wind Tunnel Facility _ . . . . 22

1.3 Research and Thesis Objectives . . . . . . . . . . . . . . 25

1.4 Thesis Structure. . . . . . . . . . . . . . . . . . . . . . 26 
\begin{tabular}{|lll}
\hline 2 & Aeroacoustic Wind Tunnel Design Methodology & 27
\end{tabular}

2.1 Design Requirements . . . . . . . . . . . . . . . . . . . . . 27

2.2 Aeroacoustic Wind Tunnel Facility Layout . . . . . . . . . . . . . . . 30

2.3 Wind Tunnel Configuration Feasibility Study . . . . . . . . . . . . . . 40

2.3 .1 Overview of the Wind Tunnel Analytical Model . . . . . . . . 41

2.3 .2 Overview of the Duct Component Flow Analysis . . . . . . . . 43

2.3.3 Wind Tunnel Feasibility Analysis Results . . . . . . . . . . . . 47

2.4 Wind Tunnel Individual Component Design . . . . . . . . . . . . . . 50

$2.4 .1 \quad$ Flexible High Pressure Air Hose . . . . . . . . . . . . . . . . . 51

2.4 .2 Round-to-Rectangular Transition Duct . . . . . . . . . . . . . 52

2.4 .3 Settling Duct and Flow Conditioning Devices . . . . . . . . 55

2.4 .4 Chamber Inlet Nozzle Section . . . . . . . . . . . . . . . . . 59

2.4 .5 Anechoic Test Chamber . . . . . . . . . . . . . . . . . . . . 67

2.4 .6 Chamber Ventilation Silencers . . . . . . . . . . . . . . . 70

2.4 .7 Test Section Jet Collector . . . . . . . . . . . . . . . . . . . . 74

2.4 .8 Collector and Vertical Exhaust Diffusers . . . . . . . . . . . . 83

$2.4 .9 \quad$ Exhaust Corner Transition and Flow Turning Vanes . . . . . . 89

$\begin{array}{|ll|}3 & \text { Fabrication of Wind Tunnel Components }\end{array}$

3.1 Anechoic Chamber . . . . . . . . . . . . . . . . . . . . . . . . . 94

3.2 Flexible High Pressure Air Hose . . . . . . . . . . . . . . . . . . . . . 96

3.3 Round-to-Rectangular Transition Duct . . . . . . . . . . . . . . . . . 97

3.4 Flow Conditioning Devices . . . . . . . . . . . . . . . . . . . . . 99

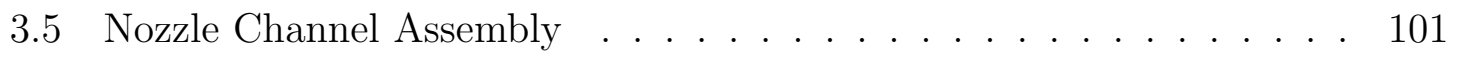

3.6 Chamber Flow Exhaust System . . . . . . . . . . . . . . . . . 103

3.7 Chamber Test Section $\ldots \ldots \ldots$. . . . . . . . . . . . . . . 108 
3.8 Acoustic Treatments . . . . . . . . . . . . . . . . . . . . . . . . . 110

$\begin{array}{|lll|}4 & \text { Experimental Methods and Facility Operation } & 112\end{array}$

4.1 Blowdown Wind Tunnel Operation . . . . . . . . . . . . . . . . . 112

$4.1 .1 \quad$ Measurement System Requirements and Locations. . . . . . . 114

4.2 Existing Facility Control System Hardware . . . . . . . . . . . . 116

4.3 Wind Tunnel Instrumentation . . . . . . . . . . . . . . . . . . . 118

4.3 .1 Control Total Pressure Pitot Probe . . . . . . . . . . . . . 120

4.3 .2 Control Static Pressure Tap . . . . . . . . . . . . . . . . . . 121

4.3 .3 Calibration Pitot-Static Probe . . . . . . . . . . . . . . . . 121

4.3 .4 Reference Absolute Pressure Transducer . . . . . . . . . . . 122

4.3 .5 Differential Pressure Transducers $\ldots \ldots \ldots$

4.3 .6 Total Temperature Thermocouple . . . . . . . . . . . . . . . 123

4.3 .7 Surface Pressure Fluctuation Sensors . . . . . . . . . . . . 123

4.3 .8 Test Section Video Camera $\ldots \ldots \ldots \ldots$

\begin{tabular}{|lll|}
5 & Calibrations and Wind Tunnel Commissioning & 126 \\
\hline
\end{tabular}

5.1 Sensor Calibrations . . . . . . . . . . . . . . . . . . 126

5.2 Control System Parameters ．. . . . . . . . . . . . . . . . . . . . 129

$5.2 .1 \quad$ Sampling Parameters . . . . . . . . . . . . . . . . . . . . . . . 129

5.2 .2 Control System Gains … . . . . . . . . . . . 130

$5.2 .3 \quad$ Control Valve Initial Threshold Voltage . . . . . . . . . . . . 131

5.2 .4 Control Mach Number Undershoot . . . . . . . . . . . . . 131

5.3 Flow Speed Calibrations . . . . . . . . . . . . . . . . . 132

5.4 Flow Characterization Data . . . . . . . . . . . . . . . . . 134

5.4 .1 Controlled Mach Number . . . . . . . . . . . . . . . . . 135

5.4 .2 Mass Flow Rate and Run Time . . . . . . . . . . . . . 138 
$5.4 .3 \quad$ Flow Temperature Drop $\ldots \ldots \ldots$. . . . . . . . . . . . . . 139

$5.5 \quad$ Measurement Uncertainty . . . . . . . . . . . . . . . . . . . . 141

5.5 .1 Reference Pressure Measurement Uncertainty . . . . . . . . . 142

5.5 .2 Total Pressure Measurement Uncertainty . . . . . . . . . . . 143

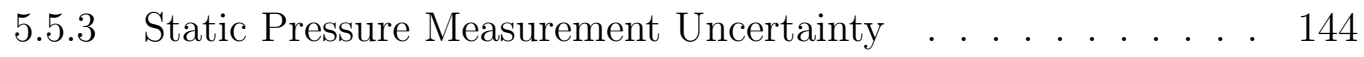

$5.5 .4 \quad$ Mach Number Uncertainty . . . . . . . . . . . . . . . . 145

6 Single-Point Surface Pressure Fluctuation Spectrum Measurements 147

$6.1 \quad$ Spectrum Models in Literature $\ldots \ldots \ldots \ldots$. . . . . . . 147

$6.1 .1 \quad$ Robertson Model (1971) [87] . . . . . . . . . . . . . . . . 148

$6.1 .2 \quad$ Efimtsov Model (1984) [89] . . . . . . . . . . . . . . . . . 148

$6.1 .3 \quad$ Laganelli Model (1993) [90] . . . . . . . . . . . . . . . . . 149

6.1 .4 Goody Model (2004) [22] . . . . . . . . . . . . . . . . . . . 149

6.1 .5 Rackl and Weston Model (2005) [93] . . . . . . . . . . . 150

$6.1 .6 \quad$ Estimation of Boundary Layer and Flow Parameters _. . . . 151

6.2 Signal Processing Methods . . . . . . . . . . . . . . . . . . . . 152

$6.2 .1 \quad$ Sampling and Signal Conditioning . . . . . . . . . . . . 153

6.2 .2 Power Spectral Density Estimation . . . . . . . . . . . . . 153

$6.3 \quad$ Chamber Background Noise Measurements _ . . . . . . . . . . . 155

$6.4 \quad$ Noise Cancellation Techniques . . . . . . . . . . . . . . . . 156

$6.5 \quad$ Spectrum Results and Discussion $\ldots \ldots \ldots \ldots \ldots \ldots$

\begin{tabular}{|lll}
\hline 7 & Conclusions and Future Recommendations & 169
\end{tabular}

7.1 Conclusions . . . . . . . . . . . . . . . . . . . . . . . . 169

7.2 Future Recommendations . . . . . . . . . . . . . . . . 171

7.2 .1 Control System and Instrumentation Improvements . . . . . . 171

7.2 .2 Recommended Flow Characterization Studies . . . . . . . . 173 
References

Appendix A Description of Computational Environment Used for Component Design Validation 185

A.1 Computer Hardware and CFD Solver . . . . . . . . . . . . . . . . . . 185

A.2 Domain Mesh Strategy and Quality Metrics . . . . . . . . . . . . . . 187

A.3 Solution Convergence and Sensitivity Studies . . . . . . . . . . . . . . 189 


\section{List of Figures}

1.1 Representation of aircraft internal noise radiation due to TBL interactions with the fuselage during cruise flight (adapted from [11]). . . . . 3

1.2 Visual representation of a turbulent boundary layer (adapted from [8]). 6

1.3 Typical non-dimensional mean velocity profile of a turbulent boundary layer (adapted from [19]). . . . . . . . . . . . . . . . . . . . . 7

$1.4 \quad$ Flat plate TBL flow problem (image from [9]). . . . . . . . . . . . . 8

1.5 Typical surface pressure fluctuation spectrum behavior beneath a turbulent boundary layer (adapted from [15]). . . . . . . . . . . . . . . 12

1.6 Visual characteristics of anechoic chambers (images from [34].) . . . . 17

1.7 Examples of anechoic wind tunnel facilities around the world - Facility schematics . . . . . . . . . . . . . . . . . . . . . . 19

1.8 Examples of anechoic wind tunnel facilities around the world: Test section views . . . . . . . . . . . . . . . . . 20

1.9 Existing Carleton HSWT facility. . . . . . . . . . . . . . . . . . . . . 22

1.10 Detailed View of Control Valve Upstream Piping. . . . . . . . . . . . 24

1.11 Straight channel assembly. . . . . . . . . . . . . . . . . . . 25

2.1 Complete Carleton HSAWT facility. . . . . . . . . . . . . . . . . 30

2.2 Carleton HSAWT alternate facility views. . . . . . . . . . . . . . . . 31

2.3 Nozzle channel assembly connection interface. . . . . . . . . . . . . . 34 
2.4 Nozzle channel assembly connection interface. . . . . . . . . . . . . . 35

2.5 Carleton HSAWT facility plan view (all dimensions in $\mathrm{mm}$ ). . . . . . 37

$2.6 \quad$ Detailed section views of the HSAWT facility. . . . . . . . . . . . . . 38

2.7 Idealized breakdown of a blowdown wind tunnel component model (adapted from [47]). . . . . . . . . . . . . . . . . . . . . . . . . . . . 41

2.8 Schematic breakdown of major wind tunnel datum stations. . . . . . 44

2.9 Required average wind tunnel mass flow rates for various governed test section Mach numbers. . . . . . . . . . . . . . . . . . . . . . . . . . . 49

2.10 Estimated available wind tunnel run time for various governed test section Mach numbers. . . . . . . . . . . . . . . . . . . . . . . . . . . 50

2.11 RN1 flexible suction/discharge hose (adapted from [51]) . . . . . . . . 51

2.12 Transition channel internal duct shape and definition (all dimensions in $\mathrm{cm}$. . . . . . . . . . . . . . . . . . 53

2.13 Comparison of divergence plane streamwise velocity contour plots Transition channel CFD validation analysis . . . . . . . . . . . . 55

2.14 Schematic of honeycomb flow conditioning cells (all dimensions in $\mathrm{mm}$ ). 57

2.15 Schematic of wire mesh flow conditioning screen. . . . . . . . . . . 57

2.16 Visualization of possible regions of separation in a general contraction profile (adapted from [44]). . . . . . . . . . . . . . . . . . 60

2.17 Schematic of cubic matched nozzle profile. . . . . . . . . . . . . 62

2.18 Nozzle channel internal duct shape and definition (all dimensions in cm). 64

2.19 Contraction plane streamwise velocity contour plot - Nozzle channel CFD validation analysis. . . . . . . . . . . . . . . . . 65

2.20 Streamwise nozzle wall parameter data - Nozzle Channel CFD validation analysis. . . . . . . . . . . . . . . 66 
2.21 Outlet centreline plane spanwise parameter data - Nozzle Channel CFD validation analysis. . . . . . . . . . . . . . 66

2.22 Test chamber plan view schematic (all dimensions in $\mathrm{m}$ ). . . . . . . . 67

2.23 Test chamber elevation view schematic (all dimensions in $\mathrm{m}$ ). . . . . . 68

2.24 General plenum silencer design configurations (adapted from [67]). . . 71

2.25 Chamber ventilation silencer design schematic (all dimensions in $\mathrm{cm}$ ). $\quad 73$

2.26 HSAWT two-pass plenum silencer design transmission loss performance predictions. . . . . . . . . . . . . . . . . . . . . 74

2.27 Diagram of general open-jet test section pressure fluctuation edge-tone feedback mechanism (taken from [72]). . . . . . . . . . . . . . . 76

2.28 NASA Langley open jet wind tunnel test section schematic (taken from [42]). . . . . . . . . . . . . . . . . . . . . . . . . . 77

2.29 Modeled domain for test section/collector CFD analyses. . . . . . . . 78

2.30 Velocity vector field plots - Preliminary collector CFD validation analysis. 79

2.31 Test chamber flow analysis plots - Preliminary collector CFD validation analysis. . . . . . . . . . . . . . . . . . . . 80

2.32 Velocity vector field plots - Final collector CFD validation analysis. . 81

2.33 Test chamber flow analysis plots - Final collector CFD validation analysis. 82

2.34 Jet collector shape with selected dimensions (all dimensions in $\mathrm{cm}$ ). . $\quad 83$

2.35 General two-dimensional flat-walled diffuser geometry. . . . . . . . . . 84

2.36 HSAWT exhaust system diffuser designs: internal duct shape and def-

inition (all dimensions in $\mathrm{cm}$ ). . . . . . . . . . . . . . . . 85

2.37 Fox and Kline [78] two-dimensional flat-walled diffuser data (design

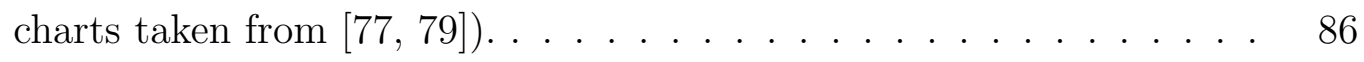

2.38 Divergence plane streamwise velocity contour plots - Diffuser channel CFD validation analysis. . . . . . . . . . . . . . . . . . . . 88 
2.39 Internal schematic of exhaust corner (all dimensions in $\mathrm{cm}$ ). . . . . . 89

2.40 Comparison of general turning vane designs. . . . . . . . . . . . . . . 90

2.41 Turning vane design profile (all dimensions in $\mathrm{cm}$ ). . . . . . . . . . . 91

2.42 Plane velocity magnitude contour plots - Turning vane CFD validation analysis. . . . . . . . . . . . . . . . . . . . . . 92

2.43 Corner Outlet centreline Velocity Profile - Turning vane CFD validation analysis. . . . . . . . . . . . . . . . . . . . . . . 93

3.1 Assembly of anechoic chamber. . . . . . . . . . . . . . . . . . . . 95

3.2 Completed anechoic chamber. . . . . . . . . . . . . . . . 96

$3.3 \quad$ Flexible high pressure air hose assembly. . . . . . . . . . . . . . . . . 97

3.4 Fabricated transition channel. . . . . . . . . . . . . . . . . . . . . . . 98

3.5 Transition channel welded component assembly. . . . . . . . . . . . . 98

3.6 Transition channel connection interfaces. . . . . . . . . . . . . . . . . 99

3.7 Upstream flow conditioning devices. . . . . . . . . . . . . . . . . 100

3.8 Downstream flow conditioning device (Screen \#2). . . . . . . . . . . 100

3.9 Nozzle channel assembly. . . . . . . . . . . . . . . . . . . . . . . . . . 101

3.10 Fabricated nozzle system. . . . . . . . . . . . . . . . . . . . 102

3.11 Nozzle feed-through hole cover. . . . . . . . . . . . . . . . . . . . 103

3.12 Complete chamber flow exhaust system model. . . . . . . . . . . . . . 104

3.13 Exhaust system major component drawings (all dim in inches). . . . . 104

3.14 Chamber internal flow exhaust component. . . . . . . . . . . . 106

3.15 Chamber external flow exhaust assembly. . . . . . . . . . . . . . . 107

3.16 Turning vane rack assembly. . . . . . . . . . . . . . . . . . . . . . . . 108

3.17 Test section TBL test panel system. . . . . . . . . . . . . . . . 109

3.18 Completed chamber test section. . . . . . . . . . . . . . . 109

3.19 Component acoustic treatments. . . . . . . . . . . . . . . . 110 
3.20 Complete anechoic test section. . . . . . . . . . . . . . . . . . . 111

4.1 Schematic of a typical idealized subsonic blowdown wind tunnel (adapted from [47]). . . . . . . . . . . . . . . . . . . . . . . . . 113

4.2 Schematic of wind tunnel measurement system locations. . . . . . . . 115

4.3 Existing wind tunnel control system (adapted from [48]). . . . . . . . 117

4.4 HSAWT instrumentation system diagram. . . . . . . . . . . . . . . . 119

4.5 Control total pressure pitot probe. . . . . . . . . . . . . . . . . . . 120

4.6 Control static pressure tap. . . . . . . . . . . . . . . . . . . . . . . . 121

4.7 Fluctuating pressure sensor schematic. . . . . . . . . . . . . . . . . . 124

4.8 TBL test panel sensor flush mounting. . . . . . . . . . . . . . . . . . 125

5.1 Reference pressure transducer calibration curve. . . . . . . . . . . . . 127

5.2 Differential pressure transducer calibration curves. . . . . . . . . . . . 128

5.3 Control total pressure calibration data. . . . . . . . . . . . . . . 133

5.4 Control static pressure tap error correction. . . . . . . . . . . . . . . 134

5.5 Comparison of measured test section Mach number to control system target for a variety of flow speeds. . . . . . . . . . . . . . . . . . . . . 135

5.6 Measured test section Mach number data - Magnified 30 second plots. 136

5.7 Wind tunnel test section flow capacity parameters. . . . . . . . . . . 139

$5.8 \quad$ Test section total temperature drop (typical 30 second run). . . . . . 140

5.9 Test section Reynold's number variation due to temperature drop (typical 30 second run). . . . . . . . . . . . . . . . . . . . . . . 141

5.10 Control system total pressure measurement uncertainty. . . . . . . . . 144

5.11 Computed control Mach number uncertainty. . . . . . . . . . . . . . 145

6.1 Comparison of overall chamber background noise levels with averaged model predictions of overall TBL signal power. . . . . . . . . . . . . . 156 
6.2 Comparison of measured single-point pressure fluctuation spectra for various test section Mach numbers. . . . . . . . . . . . . . . . . . . . 159

6.3 Comparison of normalized pressure fluctuation spectra for various test section Mach numbers. . . . . . . . . . . . . . . . . . . . . . . . . . 161

$6.4 \quad$ Estimated pressure fluctuation sensor non-dimensional diameter. . . . 163

6.5 Comparison of pressure fluctuation spectrum literature model predictions - Mach 0.74. . . . . . . . . . . . . . . . . . . . . . . . . . . . . . 164

6.6 Comparison of measured pressure fluctuation spectrum with literature models - Low Mach number range. . . . . . . . . . . . . . . . . . . . 165

6.7 Comparison of measured pressure fluctuation spectrum with literature models - Mid Mach number range. . . . . . . . . . . . . . . . . . . . 166

6.8 Comparison of measured pressure fluctuation spectrum with literature models - High Mach number range. . . . . . . . . . . . . . . . . . . . 167

6.9 Overall TBL spectral energy level comparison. . . . . . . . . . . . . . 168

$7.1 \quad$ Virginia Polytechnic Institute and State University aeroacoustic wind tunnel with closed Kevlar test section walls (adapted from [46]). . . . 172

A.1 General comparison of component domain meshing strategies. . . . . 188

A.2 General simulation solution convergence behavior (nozzle channel example). . . . . . . . . . . . . . . . . . . . . . 190

A.3 Typical flow parameter convergence behavior (nozzle channel example). 191 


\section{List of Tables}

1.1 Summary and survey of select aeroacoustic wind tunnel facilities around the world (data from [36 [46]). . . . . . . . . . . . . . . . . 21

1.2 HSWT valve specifications [48]. . . . . . . . . . . . . . . . 23

$2.1 \quad$ Summary of major HSAWT design requirements. $\ldots \ldots \ldots$

$2.2 \quad$ Summary of HSAWT major component design parameters. . . . . . . 39

2.3 Summary of wind tunnel datum station components. . . . . . . . . 45

2.4 Summary of estimated wind tunnel flow conditions - Test section Mach number: $M=0.8 . \ldots \ldots \ldots \ldots \ldots$

$2.5 \quad$ HSAWT flow conditioning screen design parameters. $\ldots \ldots \ldots$. . . 59

$2.6 \quad$ Anechoic chamber noise reduction profile (based on data from [34])] . 70

4.1 HSAWT differential pressure transducer data [85]. . . . . . . . . . . 122

4.2 Microphone sensor calibration data [17]. . . . . . . . . . . . . . . . 123

5.1 Pressure transducer calibration data. . . . . . . . . . . . . . . . . 128

5.2 Control Mach number undershoot behavior. . . . . . . . . . . . . 131

6.1 Measured flow properties within the test section. . . . . . . . . . 152 


\title{
Nomenclature
}

\author{
Abbreviations \\ ANSI American National Standards Institute \\ ASHRAE American Society of Heating, Refrigeration, and Air-Conditioning \\ Engineers \\ CFD Computational Fluid Dynamics \\ CRIAQ Consortium for Research and Innovation in Aerospace in Quebec \\ DAQ Data Acquisition \\ DFT Discrete Fourier Transform \\ DNS Direct Numerical Simulation \\ EPDM Ethylene-Propylene Rubber \\ FFT Fast Fourier Transform \\ HPF High-Pass Filter \\ HSWT High-Speed Wind Tunnel \\ HSAWT High-Speed Aeroacoustic Wind Tunnel \\ LPF Low-Pass Filter \\ MSAWT Medium-Speed Aeroacoustic Wind Tunnel \\ NPT National Pipe Thread \\ NSERC National Sciences and Engineering Research Council \\ PID Proportional-Integral-Derivative
}




$\begin{array}{ll}\text { PSD } & \text { Power Spectral Density } \\ \text { RANS } & \text { Reynolds-Averaged Navier-Stokes } \\ \text { RMS } & \text { Root-Mean-Square } \\ \text { SNR } & \text { Signal-to-Nose Ratio } \\ \text { OASPL } & \text { Overall Sound Pressure Level } \\ \text { SST } & \text { Shear-Stress Transport } \\ \text { TBL } & \text { Turbulent Boundary Layer } \\ \text { ZMPG } & \text { Zero Mean Pressure Gradient }\end{array}$

\section{Symbols}

$\begin{array}{ll}A & \text { Local Channel Duct Area }[\mathrm{m}] \\ A_{\text {cal }} & \text { Linear Calibration Slope } \\ A R & \text { Ratio of Diffuser Outlet Area to Inlet } \\ B_{\text {cal }}\left(B_{\text {offset }}\right) & \text { Linear Calibration Offset } \\ b_{d} & \text { Two-Dimensional Diffuser Duct Constant Depth }[\mathrm{cm}] \\ c & \text { Turning Vane Chord Length }[\mathrm{m}] \\ C_{f} & \text { Skin-Friction Coefficient } \\ C R & \text { Ratio of Contraction Duct Inlet Area to Outlet Area } \\ d & \text { Duct Diameter }[\mathrm{m}] \\ d_{m i c} & \text { Pressure Fluctuation Sensor Diameter }[\mathrm{m}] \\ d_{m i c}^{+} & \text {Pressure Fluctuation Sensor Non-Dimensional Diameter } \\ f & \text { Cyclic Frequency }[H z] \\ f_{s} & \text { Sampling Rate }[H z] \\ F_{c} & \text { Laganelli Spectrum Model Compressible Transformation Function } \\ F_{e} & \text { Morel Nozzle Duct Exit Design Parameter } \\ G_{i} & \text { Morel Nozzle Duct Inlet Design Parameter }\end{array}$




\begin{tabular}{|c|c|}
\hline$h / c$ & Turning Vane Spacing-to-Chord Ratio \\
\hline$h_{w} / h_{a w}$ & Specific Adiabatic Wall Enthalpy Ratio \\
\hline$i$ & Imaginary Unit $[\sqrt{-1}]$ \\
\hline K & Duct Component Pressure Loss Coefficient \\
\hline$K_{D}$ & Control System Derivative Gain \\
\hline$K_{I}$ & Control System Integral Gain \\
\hline$K_{P}$ & Control System Proportional Gain \\
\hline$L_{d}$ & Diffuser Duct Length $[\mathrm{cm}]$ \\
\hline$L_{n}$ & Nozzle Duct Length $[\mathrm{cm}]$ \\
\hline$L_{p}$ & Sound Pressure Level $[d B]$ \\
\hline$M$ & Mach Number \\
\hline$\dot{m}$ & Mass Flow Rate $[\mathrm{kg} / \mathrm{s}]$ \\
\hline$O A$ & Perforated Sheet Metal Open Area Ratio [\%] \\
\hline$p^{\prime}$ & Local Surface Pressure Fluctuations $[\mathrm{Pa}]$ \\
\hline$p_{\text {rms }}$ & Root-Mean-Square of Pressure Fluctuations $[\mathrm{Pa}]$ \\
\hline$p_{\text {ref }}$ & Reference Sound Pressure $[\mathrm{Pa}]$ \\
\hline$P$ & Static Pressure $[P a]$ \\
\hline$P_{o}$ & Total Pressure $[P a]$ \\
\hline$q$ & Dynamic Pressure $[\mathrm{Pa}]$ \\
\hline$r$ & Turbulent Recovery Factor \\
\hline$R$ & Specific Gas Constant for Air $\left[\mathrm{Jkg}^{-1} \mathrm{~K}^{-1}\right]$ \\
\hline$R_{p}$ & Auto-Correlation of Surface Pressure Fluctuations $\left[\mathrm{Pa}^{2}\right]$ \\
\hline$R_{T}$ & Pressure Fluctuation Timescale Ratio \\
\hline$R e_{\tau}$ & Reynolds Number based on Friction Velocity \\
\hline$R e_{\tau o}$ & Characteristic Friction Reynolds Number \\
\hline$R e_{\theta}$ & Reynolds Number based on Momentum Thickness \\
\hline
\end{tabular}




\begin{tabular}{|c|c|}
\hline$R e_{x}$ & Reynolds Number based on Boundary Layer Growth Distance \\
\hline$s$ & Perforated sheet Metal Hole Spacing $[i n]$ \\
\hline$S$ & Flow Conditioning Screen Cell Opening Size $[\mathrm{mm}]$ \\
\hline$S t$ & Strouhal Number \\
\hline$t$ & Time $[s]$ \\
\hline$t_{\text {perf }}$ & Perforated Sheet Metal Thickness $[i n]$ \\
\hline$t / c$ & Turning Vane Thickness-to-Chord Ratio \\
\hline$T$ & Static Temperature $[K]$ \\
\hline$T_{o}$ & Total Temperature $[K]$ \\
\hline$T i$ & Perforated Sheet Metal Acoustic Transparency Index \\
\hline$T I$ & Turbulence Intensity [\%] \\
\hline$T L$ & Acoustic Transmission Loss $[d B]$ \\
\hline$u$ & Local Mean Streamwise Velocity $[\mathrm{m} / \mathrm{s}]$ \\
\hline$u^{+}$ & Non-Dimensional Local Mean Streamwise Velocity \\
\hline$u_{\tau}$ & Friction Velocity $[\mathrm{m} / \mathrm{s}]$ \\
\hline$U_{\infty}$ & Free Stream Mean Velocity $[\mathrm{m} / \mathrm{s}]$ \\
\hline$\hat{U}$ & Window Function Power Normalization Factor \\
\hline$V_{t}$ & Compressed Air Storage Tank Total Volume $\left[\mathrm{m}^{3}\right]$ \\
\hline$w$ & Flow Conditioning Screen Wire Diameter $[\mathrm{m}]$ \\
\hline $\mathrm{w}[n]$ & Discrete Signal Windowing Function \\
\hline$W_{d_{e}}$ & Two-Dimensional Diffuser Duct Exit Width $[\mathrm{cm}]$ \\
\hline$W_{d_{i}}$ & Two-Dimensional Diffuser Duct Inlet Width $[\mathrm{cm}]$ \\
\hline$W_{n_{e}}$ & Nozzle Duct Exit Width $[\mathrm{cm}]$ \\
\hline$W_{n_{i}}$ & Nozzle Duct Inlet Width $[\mathrm{cm}]$ \\
\hline$x$ & Boundary Layer Growth Distance $[m]$ \\
\hline$X_{m}$ & Nozzle Polynomial Match Point Location [fraction of $L_{n}$ ] \\
\hline
\end{tabular}




\section{Greek}

\begin{tabular}{|c|c|}
\hline$\alpha$ & Efimstov Spectrum Model Constant \\
\hline$\beta$ & Efimstov Spectrum Model Constant \\
\hline$\gamma$ & Ratio of Specific Heats for Air \\
\hline$\delta$ & Boundary Layer Thickness $[m]$ \\
\hline$\delta^{*}$ & Boundary Layer Displacement Thickness $[m]$ \\
\hline$\delta F$ & Uncertainty in Parameter, $F$ \\
\hline$\theta$ & Boundary Layer Momentum Thickness $[m]$ \\
\hline$\theta_{\text {diff }}$ & Diffuser Included Wall Divergence Angle [º] \\
\hline$\mu$ & Dynamic Viscosity $[k g / m-s]$ \\
\hline$\nu$ & Kinematic Viscosity $\left[\mathrm{m}^{2} / \mathrm{s}\right]$ \\
\hline$\rho$ & Density $\left[\mathrm{kg} / \mathrm{m}^{3}\right]$ \\
\hline$\sigma$ & Flow Conditioning Screen Open Area Ratio \\
\hline$\tau$ & Time Delay $[s]$ \\
\hline$\tau_{w}$ & Wall Shear Stress $[P a]$ \\
\hline$\Upsilon_{p r}$ & Cross-spectrum between signals at points $\mathrm{p}$ and $\mathrm{r}\left[\mathrm{Pa}^{2} / \mathrm{Hz}\right]$ \\
\hline$\Phi_{p}(f)$ & Single-Sided Surface Pressure Fluctuation Spectrum $\left[\mathrm{Pa}^{2} / \mathrm{Hz}\right]$ \\
\hline$\widehat{\Phi_{p}}(f)$ & $\begin{array}{l}\text { Single-Sided Surface Pressure Fluctuation Spectrum Estimate } \\
{\left[\mathrm{Pa}^{2} / \mathrm{Hz}\right]}\end{array}$ \\
\hline
\end{tabular}

$\Phi_{p}(\omega)$

$\Phi_{p p}(\omega)$

$\Psi[k]$

$\omega$
Wall-Normal Distance within Boundary Layer $[m]$

Normalized Wall-Normal Distance within Boundary Layer 


\section{Chapter 1}

\section{Introduction}

This thesis outlines the work conducted in the design, fabrication, and commissioning of the new High-Speed Aeroacoustic Wind Tunnel (HSAWT) at Carleton University. This new wind tunnel is a facility upgrade to the current high-speed wind tunnel, with collaborative funding and partnership from the Consortium for Research and Innovation in Aerospace in Quebec (CRIAQ) and the National Sciences and Engineering Research Council (NSERC). The purpose of this wind tunnel is to facilitate current and future research initiatives in developing experimental techniques for studies of aerodynamically induced noise phenomena; with a particular focus on Turbulent Boundary Layer (TBL) induced noise generated by aircraft structures in flight.

The first chapter of this thesis details the motivation behind this research endeavor, followed by a review of studies of TBL induced noise in literature. A description of general aeroacoustic wind tunnel design methodology is then provided, as well as a description of the current high-speed wind tunnel facility at Carleton. Finally, the overall research objectives of this work are outlined, along with the overall structure of the presented thesis. 


\subsection{Motivation}

Aeroacoustics, or the study of aerodynamic and turbulence generated noise, has become an important research initiative over the span of the last 60 years due to the ever increasing capabilities of aerodynamic vehicles and power-plant. Sound generation from turbulent flow occurs due to the nature of the fluctuating vorticity and velocity field, resulting in fluctuations of pressure. This phenomena leads to the propagation of acoustic waves whose disturbances reach the ear for interpretation as sound. Sound generated by turbulence is generally unwanted in almost all applications; hence it is commonly characterized as noise [1-3].

A significant engineering problem in the realm of aeroacoustics involves the development of the TBL over the surfaces of aircraft at high flow speeds. During cruise flight, the main source of inward acoustic noise radiation within the aircraft cabin occurs as a result of the random pressure fluctuation field imposed by the TBL [4] 6 . This acoustic noise transmission occurs on two fronts. The first involves the aerodynamic component of noise transmission from the random fluctuating pressure field itself. The second, and possibly more important, involves the structure borne component of noise transmission due to the excitation of the structural fuselage panels from the fluctuating pressure field; resulting in a vibro-acoustic response from the light-weight, flexible panels and consequential production of acoustic radiation into the aircraft cabin [7-10]. Moreover, the radiated sound pressure levels can be increasingly significant during high sub-sonic flight speeds when the convective speed of the coherent turbulent structures within the TBL matches the trace speed of a resonate structural mode of the fuselage panel; a phenomena termed as aerodynamic coincidence which entails large panel vibrations and levels of cabin noise radiation (refer to Figure 1.1). 


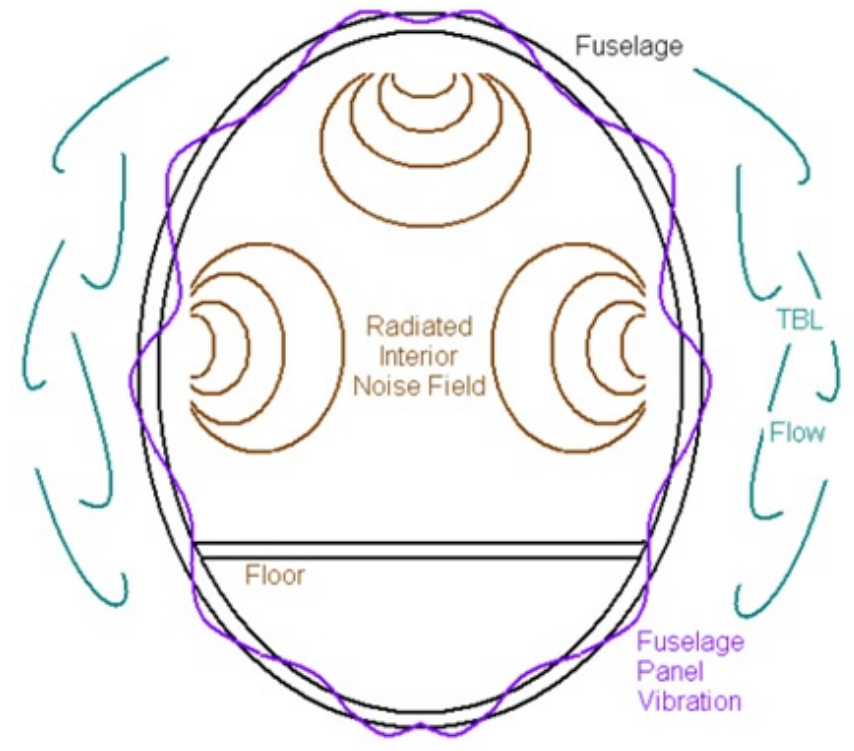

Figure 1.1: Representation of aircraft internal noise radiation due to TBL interactions with the fuselage during cruise flight (adapted from [11]).

The major implications of the interactions between the TBL and the aircraft structure during cruise flight are two-fold: one being the propagation of unwanted noise within the interior of the cabin and the other being the resulting acoustic fatigue stresses in all of the affected structures. With regards to the former, the radiated acoustic sound pressure levels can be intense and have been shown to have a large effect on passenger/crew discomfort, communication interference, and malfunction of vital sensitive electronic equipment [12]. Long-term exposure to large levels of low frequency noise has also been shown to increase the likelihood of developing certain cardiovascular diseases [13]. Some airlines therefore require manufacturers to ensure that certain cabin noise levels are not exceeded [8]. Due to the increasing flight speed capabilities of business and commercial aircraft, the resulting relative increase in cabin noise levels has led to a desired need for a better understanding of TBL interactions with the exterior of aircraft, so that optimal panel design strategies can be implemented to help mitigate large structural excitations and radiated sound. 
Conventional methods of describing the interactions between TBL induced surface pressure fluctuations and the structural response of the flexible fuselage panels have relied on the use of statistical semi-empirical models based on theory and experimental measurements; since describing this phenomenon through numerical simulations of high Reynolds number fluid flow coupled with structural excitation and acoustic noise transmission is both extremely time consuming and computationally expensive. A basic assumption in this analysis is that the pressure fluctuations inside the boundary layer excite the flexible structures, in which their relatively small vibration amplitudes have negligible effects on the boundary layer or flow parameters themselves. This means that the problem can be divided into a separate analysis of the flow induced pressure fluctuations acting on a rigid surface and the subsequent response of the flexible panel when the excitation forcing function of the fluctuations is applied. The modeled response of the panel can then be used in radiated sound power models which predict the interior sound pressure levels induced by the vibrations of the panel interacting with the still air inside the cabin.

There have been many studies in literature involving the development of semiempirical models used to characterize TBL surface pressure fluctuations over surfaces as summarized by the authors [7, 9, 14, 15]. These models relate flow and boundary layer parameters to the average statistical properties of the energy spectrum of the random pressure fluctuations beneath the TBL. Since these models are developed from both theoretical analysis and physical measurements with varying experimental conditions, each model makes fairly different predictions with regards to the behavior of the spectrum in the various frequency regions and the contributions of the certain sizes of coherent turbulent structures within the boundary layer that influence the overall spectral energy in varying frequency regions. Furthermore, there is some inconsistency with regards to the flow and boundary layer parameters in which each model 
predicts to have the most influence on the spectrum. There is therefore a need to not only assess these inconsistencies with experimental methods that cover a wide scope of flow conditions and speeds, but also to clarify the fundamental physical relationship between the turbulent structures within the boundary layer and their contributions to the pressure fluctuation signature. Carleton University has dedicated resources to this area of research through statistical studies of TBL pressure fluctuation phenomena through experimental measurements in low-speed environments using the Medium Speed Aeroacoustic Wind Tunnel Facility (MSAWT) [16, 17]. The next phase of this research initiative, and the ultimate focus of this thesis, involves expanding Carelton's capabilities through the design and fabrication of an experimental platform capable of studying the nature of surface pressure fluctuations at a wide range of subsonic flow speeds; including those that are more representative of typical cruise flight conditions of general business and commercial aircraft. The implementation of the HSAWT at Carleton seeks to not only provide a versatile aeroacoustic test facility that is unique among many similar facilities around the world, but a test bed that can provide the opportunity to conduct quality studies in TBL surface pressure fluctuations. It is envisioned that this facility will help mitigate the need for expensive flight tests to evaluate the designs of aircraft fuselage panel structures.

\subsection{Background}

This section presents a more detailed overview of TBL surface pressure fluctuation studies in literature to provide a necessary context in terms of the influences for the purpose and design of the HSAWT facility. A discussion on the general design and purpose of aeroacoustic wind tunnel facilities is followed, along with a description of the current high-speed wind tunnel facility at Carleton and the plan to upgrade the 
facility for aeroacoustic measurement capabilities.

\subsubsection{Review of TBL Surface Pressure Fluctuation Studies}

\section{Turbulent Boundary Layers}

Wall bounded flows are typically characterized by the development of a boundary layer, in which the region of flow near the wall is separated into two distinct regions. The first contains the boundary layer itself, with its mean velocity profile starting from zero at the wall (no slip condition) to the free stream mean velocity of the flow, $U_{\infty}$. The second region represents the non-turbulent free stream which is separated by the very thin turbulent to non-turbulent interface. The time averaged distance of this interface from the wall is the boundary layer thickness, $\delta$ (See Figure 1.2).

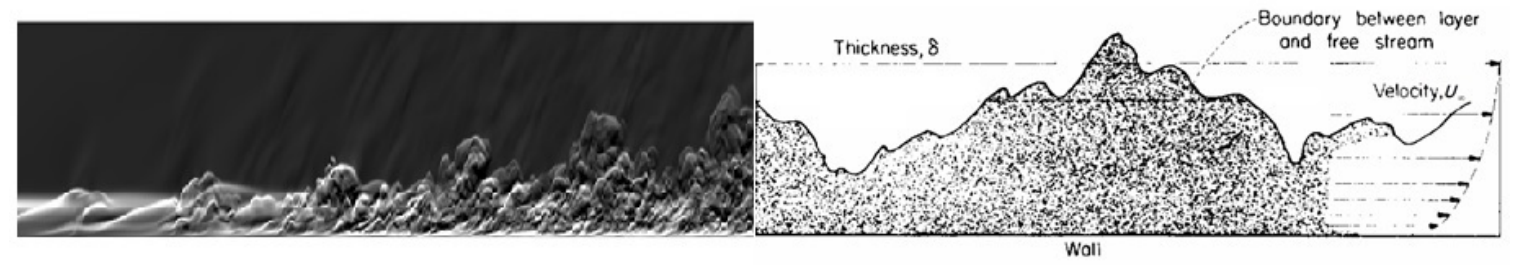

Figure 1.2: Visual representation of a turbulent boundary layer (adapted from [8]).

A typical boundary layer contains distinct characteristic regions as a function of the distance from the wall, $y$. These regions or layers can be visualized from the nondimensional mean velocity profile, $u^{+}=u / u_{\tau}$, of the boundary layer as a function of the non-dimensional wall normal distance, $y^{+}=y u_{\tau} / \nu$. This typical velocity profile is shown in Figure 1.3 for varying friction Reynolds numbers, $R e_{\tau}=u_{\tau} \delta / \nu$. The boundary layer as a whole can be separated into an inner region and an outer region, where the turbulent motions within the inner region of the boundary layer are severely impacted by friction and viscous shear stresses compared to turbulent Reynolds stresses. The case is reversed for the outer region of the boundary layer 
where viscous stresses start to become negligible further away from the wall. The various layers within the boundary layer denote distinct behavior in the velocity profile, which can be attributed to the effects of the complex turbulence generation and dissipation mechanisms from the coherent 'hairpin' vortex structures that are developed within the boundary layer. The production of these turbulent structures occurs due to a instability mechanism from a perturbation near the wall, resulting in self-sustaining packets of turbulent structures which accounts for the velocity profile behavior seen in the logarithmic layer. It is also important to note that high Reynolds number flows extend the logarithmic and wake regions of the profile, creating more pronounced and distinct regions within the boundary layer [18].

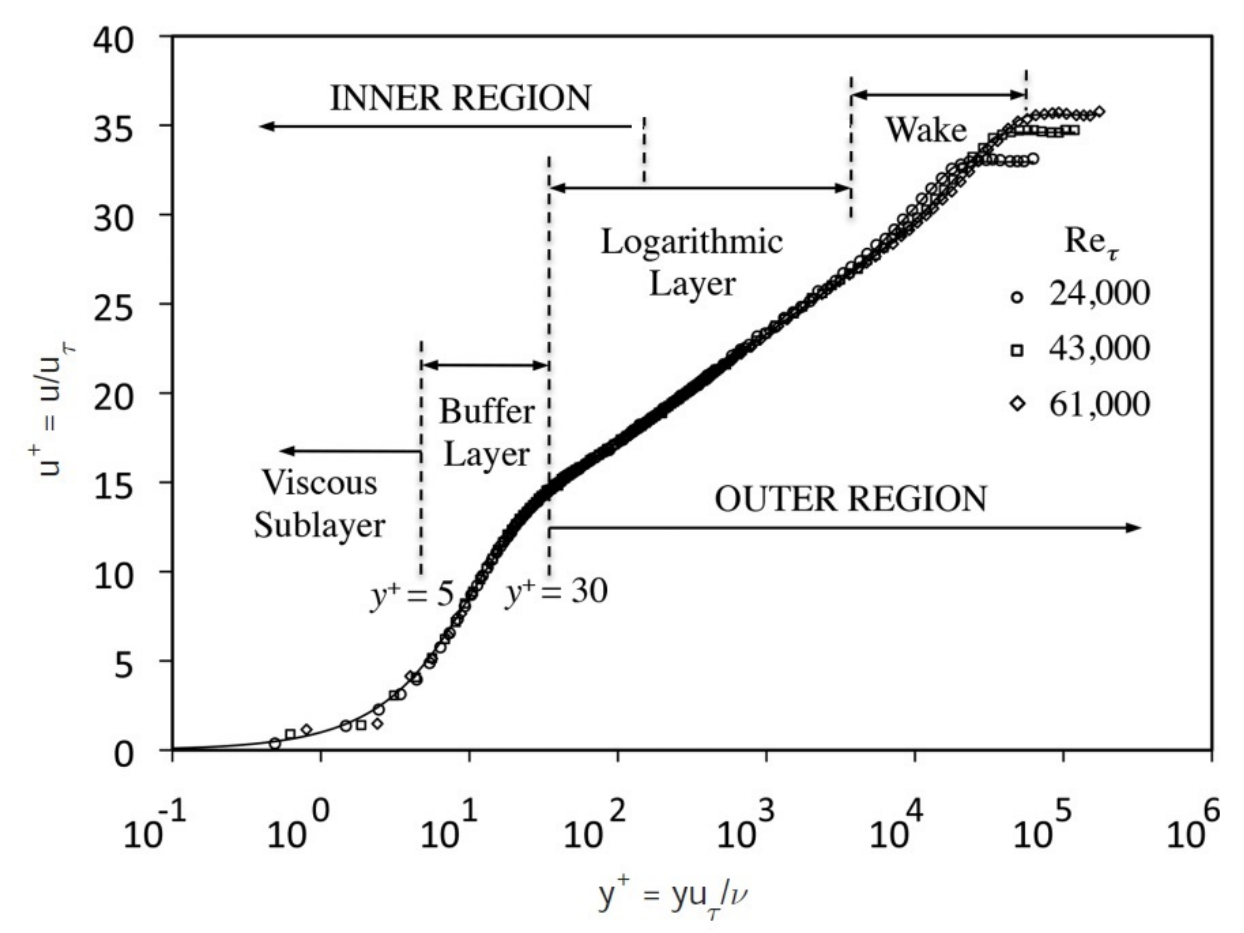

Figure 1.3: Typical non-dimensional mean velocity profile of a turbulent boundary layer (adapted from [19]). 


\section{TBL Surface Pressure Fluctuation Field Characterization}

Figure 1.4 contains a schematic of the flow field of interest which involves the development of a TBL over a flat plate. This represents a somewhat idealized case of the real flow conditions over an aircraft fuselage skin panel, in which the following assumptions apply: the TBL develops over an extended flat rigid surface with a zero-mean pressure gradient, the TBL is statistically homogeneous along the plane parallel to the boundary surface; and the TBL is weakly stationary in time [7, 8$]$.

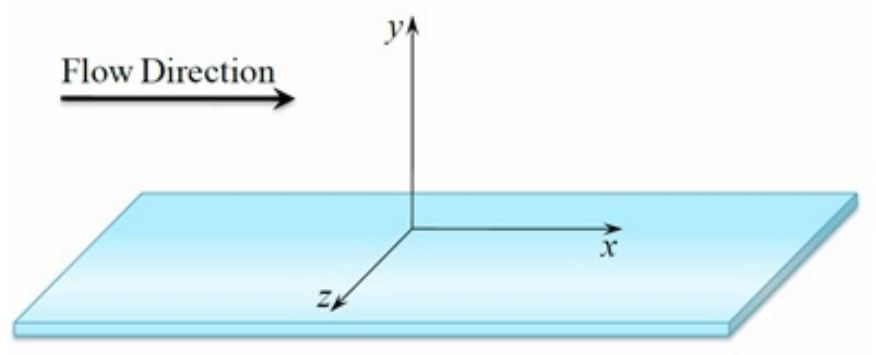

Figure 1.4: Flat plate TBL flow problem (image from [9]).

The coupling between the turbulent velocity field from the boundary layer and the induced surface pressure fluctuation field on the wall is quite complex. The integral solution for the pressure fluctuations implies a dependence on contributions from both mean shear velocity interactions with the turbulent velocity fluctuations and from the self-turbulent interactions of the velocity fluctuation products or Reynolds stresses [8]. A solution to the pressure fluctuation field pertaining to this type of problem can be determined through analytical solutions to the Reynolds-Averaged Navier-Stokes (RANS) equations by imposing simplifications and boundary conditions in light of the aforementioned assumptions, however this does not allow for complete statistical characterization of the nature of the pressure fluctuations [7, 8, 10, 20, Taking the ap-

proach of using full Direct Numerical Simulations (DNS) also has limitations in terms 
of large and impractical computational expenses with regards to analyzing complex flow problems of high Reynolds number [20]. Characterization of surface pressure fluctuations through statistical semi-empirical models developed from experimental data is thus the preferred method. Techniques for these types of measurements usually involve using arrays of flush mounted pressure sensors, with high spatial and temporal resolution, in test panels within wind tunnel or actual aircraft environments.

There are many important statistical tools for characterizing the nature of surface pressure fluctuations beneath a TBL. Historically, single-point spectral analysis of pressure fluctuations at the surface of flat panels has been an important area of research, since developed models of the spectral behavior are used as direct forcing function inputs to aircraft panel structural response and radiated noise prediction models. In the case of pressure fluctuation measurements, especially for those conducted in wind tunnel environments, careful experimental control can yield an ergodic random process. This can lead to a valuable statistical assumption, in which the ensemble average of a time varying signal, $\langle A(t)\rangle$, can be replaced by a time average of a single realization of data [21]. This allows for the calculation of the single-point surface pressure fluctuation spectrum using the auto-correlation of a time varying pressure fluctuation signal, $p^{\prime}(t)$ [7]:

$$
R_{p}(\tau)=\left\langle p^{\prime}(t) p^{\prime}(t+\tau)\right\rangle
$$

The single-point frequency spectrum of the pressure fluctuations can then be determined from a temporal Fourier Transform of the auto-correlation function [7]:

$$
\Phi_{p p}(\omega)=\frac{1}{2 \pi} \int_{-\infty}^{\infty} R_{p}(\tau) e^{-i \omega \tau} d \tau
$$

which describes the spectral density of pressure fluctuation energy beneath the TBL. 
It is important to note that this definition of the spectrum is a classical signal processing definition in which the total energy of the signal is contained within all frequencies ranging from $-\infty \leq \omega \leq \infty$; thus the spectrum is defined as two-sided [21]. Integrating the pressure fluctuation spectrum over the entire frequency range will yield the root-mean-square of the pressure fluctuations, which is a statistical metric used to collapse the entire spectrum into a singular value which represents the total mean pressure fluctuation energy beneath the TBL $[8]$ :

$$
p_{r m s}=\sqrt{\left\langle p^{\prime 2}\right\rangle}=\sqrt{R_{p}(0)}=\sqrt{\int_{-\infty}^{\infty} \Phi_{p p}(\omega) d \omega}
$$

The definition of the pressure fluctuation spectrum in Equation 1.2, $\Phi_{p p}(\omega)$, describes the spectrum as a function of angular frequency, $\omega$, for the extended range of all positive and negative frequencies (two-sided spectrum). The power spectrum is also normalized by a factor of $2 \pi$ radians/second, thus the spectrum can also be interpreted as a Power Spectral Density (PSD) function with units of mean square pressure per unit of angular frequency. It is common in literature to define the PSD in terms of only the positive frequency range, where the entire energy of the spectrum is collapsed within $0 \leq \omega \leq \infty$; thus defining the spectrum as one-sided [21]. Since the PSD is an even mathematical function, the difference in the magnitudes between these two definitions of the spectrum is shown in Equation 1.4 below:

$$
\Phi_{p}(\omega)=2 \Phi_{p p}(\omega)
$$

where slight notation differences are used; $\Phi_{p p}$ denotes the two-sided spectrum and $\Phi_{p}$ represents the one-sided spectrum. The addition of the factor of 2 ensures that the total mean pressure fluctuation energy remains the same when both spectral functions are integrated over their applicable frequency ranges: 


$$
p_{r m s}=\sqrt{\left\langle p^{\prime 2}\right\rangle}=\sqrt{\int_{-\infty}^{\infty} \Phi_{p p}(\omega) d \omega}=\sqrt{\int_{0}^{\infty} \Phi_{p}(\omega) d \omega}
$$

The single-sided spectrum, $\Phi_{p}(\omega)$, is the common representation of the PSD as a function of angular frequency in literature. This definition is useful for analytical investigations, however it is limited in experimental analysis where frequency is commonly defined in terms of cyclic frequency, $f$, in hertz (cycles per second). Equation 1.6 displays the magnitude conversion between $\Phi_{p}(\omega)$ and $\Phi_{p}(f)$ :

$$
\Phi_{p}(f)=2 \pi \Phi_{p}(\omega)
$$

where the factor of $2 \pi$ is used to account for the change in the magnitude of the spectrum due to the normalization factor. The relationship $(\omega=2 \pi f)$ can then be used to convert the angular frequency variable in $\Phi_{p}(\omega)$ to get the spectral representation in terms of cyclic frequency. Again, integration of any definition of the power spectrum over its applicable frequency range should yield the same value for the total mean pressure fluctuation energy:

$$
p_{\text {rms }}=\sqrt{\left\langle p^{\prime 2}\right\rangle}=\sqrt{\int_{0}^{\infty} \Phi_{p}(\omega) d \omega}=\sqrt{\int_{0}^{\infty} \Phi_{p}(f) d f}
$$

\section{Single-Point Surface Pressure Fluctuation Spectral Behavior}

The general behavior of the normalized pressure fluctuation frequency spectrum is shown in Figure 1.5. It is important to note that the complex nature of the TBL results in no single universal set of scaling parameters for both pressure and frequency. The TBL exhibits self-similarity within certain regions and thus the frequency components of the spectrum that are most influenced by these regions, containing certain 
sizes of turbulent eddy structures, can be scaled using parameters that best characterize the flow within those regions [7, 8, 22, 23]. There is general agreement in literature that the frequency spectrum can be divided into the four major frequency regions displayed in Figure 1.5 .

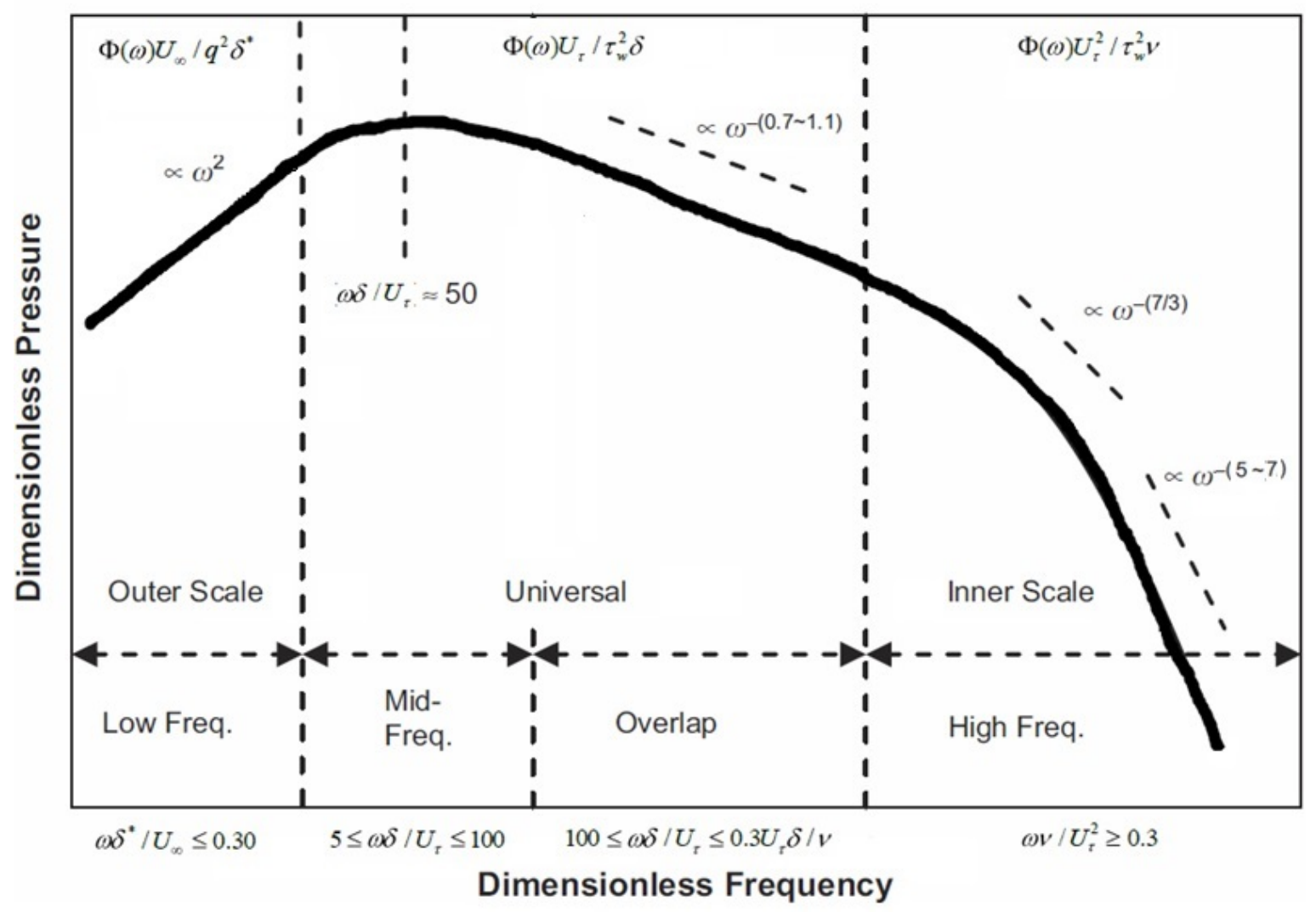

Figure 1.5: Typical surface pressure fluctuation spectrum behavior beneath a turbulent boundary layer (adapted from [15]).

The contribution to the low frequency pressure fluctuation energy comes from large scale eddies found in the outer log-law/wake region of the TBL, thus the frequency and pressure are best normalized using boundary layer parameters associated with this region (freestream velocity, $U_{\infty}$; dynamic pressure, $q$; and TBL displacement thickness, $\delta^{*}$ [7]. Low frequency measurements are difficult to obtain due to the fact the contributions from the TBL are small and usually masked by other unwanted low 
frequency noise content measured, such as structural vibrations or contaminant noise from other wind tunnel components. Despite this fact, it is acknowledged in general that the spectrum increases proportional to the square of the frequency, $\omega^{2}$. FfowcsWilliams [24] and Panton [25] first predicted a $\omega^{2}$ dependence on the spectrum at low frequencies. Low noise wind tunnel test data from Farabee [23] and Goody [22] have validated this behavior using special noise cancellation techniques to properly isolate the low frequency portion of the spectrum. A recent analysis of flight test data, free from low frequency noise contamination, by Palumbo [26] has also verified this spectral behavior within the low frequency region. It is for this reason that wind tunnel experimental measurements of the spectrum must be conducted in a low-noise environment where the low frequency component of the pressure signal represents only contributions from the TBL. This is an important aspect to consider in the design of a wind tunnel to conduct these measurements in facilities with high background noise. Another interesting feature of the spectrum is that it does not tend to zero as frequency goes to zero due to finite Mach number and compressibility effects. This represents the contribution from plane acoustic waves to the spectral energy which is a function of the fluid medium [7].

The mid- and overlap frequency regions of the spectrum are influenced by midscale turbulent structures mainly within the log-law layer of the TBL. Therefore, this region is characterized as a universal range that is roughly scale independent. Typically this region is normalized by parameters which influence both the inner and outer regions of the boundary layer (TBL thickness, $\delta$; friction velocity, $u_{\tau}$; and wall shear stress, $\left.\tau_{w}\right)$ [7]. The mid-frequency region contains an energy peak, which has been found to occur around $\omega \delta / u_{\tau}=50$ [23, [27, 28]. Theoretically, it is generally accepted that the spectrum should start to attenuate according to an approximate $\omega^{-1}$ power law after the spectral peak [7, 25, 27, 29]. Verification of this behavior 
however has proven to be difficult, with experimental spectra displaying decaying ranges from $\omega^{-0.7}$ to $\omega^{-1.1}$ from numerous authors [8, 23, 26, 30].

The high frequency behavior of the spectrum is dictated by contributions from the inner viscous sub-layer of the TBL where the small-scale turbulent structures lie. It is universally accepted that viscous effects dominate in this region and thus the spectrum best collapses when scaled based on kinematic viscosity, $\nu$, and other inner layer variables (wall shear stress, $\tau_{w}$; and friction velocity, $u_{\tau}$ ) [7]. Early measurements conducted by Schewe [31] within this frequency region indicated a dependence of the spectrum on $\omega^{-7 / 3}$, however more recent measurements with smaller transducer sizes have shown an attenuation slope ranging from $\omega^{-5}$ to $\omega^{-7}$ for very high frequencies [8, 17, 22, 23, 26, 28, 30]. The transition from the $\omega^{-7 / 3}$ minor decay region to the larger decay region seems to relate to the transition from the buffer layer to the viscous sub-layer within the TBL [17]. There is not much consensus in literature with regards to the behavior of the spectrum in the high frequency region, as opposed to other regions, due to the fact that sensors with finite transducer sizes act as low-pass filters in terms of their frequency content measurement capabilities. This is because their response is proportional to the average fluctuating pressure reading over its surface. This imposes limits for obtaining high frequency measurements and transducer size becomes extremely important in terms of measuring the correct behavior of the high frequency spectrum as freestream velocity is increased. Another important factor to consider for high frequency measurements is the configuration of the sensor and its geometry, as various configurations in measurement devices can affect the local flow at the small-scale level where the high-frequency component is most influenced, thus introducing measurement errors and discrepancies [7].

Understanding the behavior of the single-point spectrum is crucial in examining how certain regions and sizes of coherent turbulent structures within the boundary 
layer influence the fluctuating pressure signal and thus structural response of the panel. Many authors in literature have made numerous attempts at modeling the spectral behavior of the surface pressure fluctuations to serve as inputs to panel structural response and noise prediction models. The issue regarding the use of these models, which are summarized mathematically later in Chapter 6, is that they are somewhat inconsistent with regards to their predictions of the magnitude and behavior of the spectrum in the aforementioned main frequency regions. This makes sense, due to the fact that these models rely heavily on empirical measurements to extract and model the spectrum. Furthermore, the vast majority of measurements in literature do not show complete consensus in terms of spectral behavior due to varying instrumentation techniques and flow conditions examined. It is therefore important to investigate the validity of using the various developed models for accurate predictions of the TBL induced pressure fluctuation signature over a wide range of imposed flow conditions (low to high transonic speeds) and measurement techniques. The design and implementation of the HSAWT at Carleton is envisioned to facilitate this task and is the ultimate purpose of this work.

\subsubsection{Aeroacoustic Wind Tunnel Design and Survey of Ex- isting Facilities}

Aeroacoustic test facilities come in varying configurations with a vast range of capabilities. The main purpose of an aeroacoustic wind tunnel is similar to conventional wind tunnels with regards to providing a high quality flow environment to simulate the real physics of test subjects exposed to flow during physical operation. The major difference lies in the requirement for a test section/environment that is also suitable for quality measurements of the aerodynamic noise signature generated by 
test subjects when exposed to the flow. For this reason, aeroacoustic wind tunnels are usually equipped with a test section that can help minimize background noise levels and reverberations of acoustic waves which contaminate the signal of the desired noise and pressure fluctuation measurements. Wind tunnels with open-jet test sections enclosed within an anechoic test chamber are commonly implemented to facilitate this requirement. Closed-walled test sections surrounded by an anechoic chamber are also possible with the use of acoustic kevlar walls. These walls allow sound to freely pass through with minimal attenuation and reverberation to then be absorbed by the surrounding chamber walls [32, 33]. Anechoic chambers are enclosures with panelized walls (usually 4 inch thick acoustic panels) which are lined with sound attenuating material which is designed to minimize noise background levels inside the chamber. These chambers are also lined with acoustic wedges on the interior which help suppress sound reverberation to simulate an acoustic free-field environment [34]. Anechoic test chambers can be lined with acoustic wedges around all walls, termed fully-anechoic, or can have a flat floor surface which is just lined with sound absorptive material, termed hemi-anechoic. Figure 1.6 displays a typical fully-anechoic chamber lined with typical perforated sheet metal wedges.

Since aeroacoustic wind tunnels generally contain an open-jet test section enclosed by an anechoic chamber, the simulated acoustic free-field and low background noise environment allows for measurements of aerodynamically induced noise from structures exposed to flow from the tunnel with minimal noise contributions from other sources; such as the mechanical components used to drive the flow through the tunnel. This provides a good environment for the study of common aeroacoustic engineering problems such as TBL induced noise, airfoil/propeller trailing edge noise, jet/shear layer noise, and automobile component generated noise to name a 


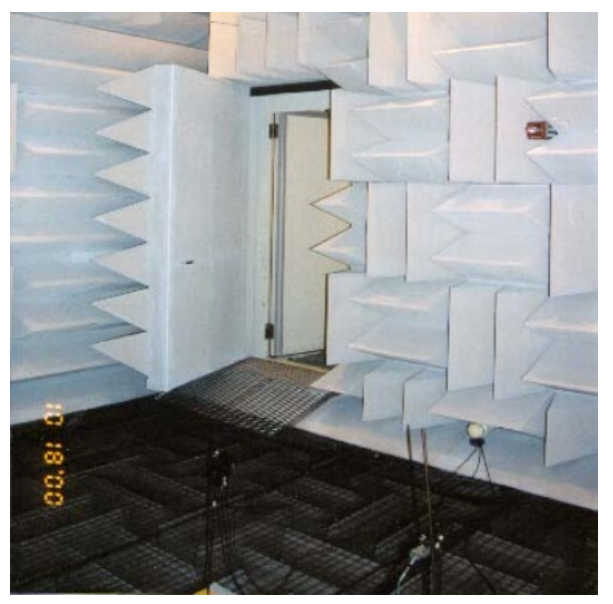

(a) Fully-anechoic chamber

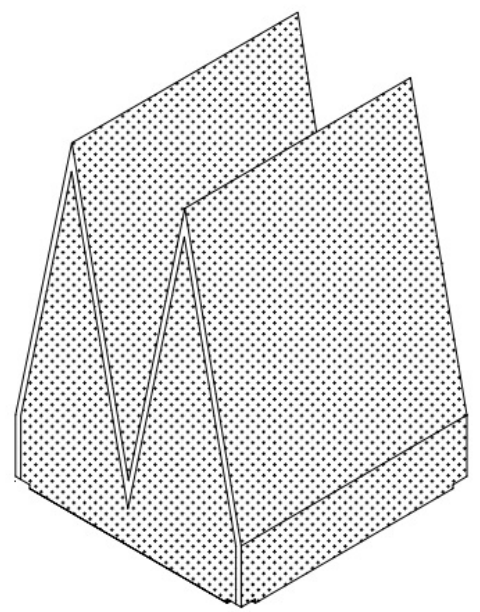

(b) Anechoic chamber wedges

Figure 1.6: Visual characteristics of anechoic chambers (images from [34.)

few. There are several automobile/aircraft manufacturers and research institutions around the world which house aeroacoustic wind tunnel test facilities to perform this type of research. These facilities are usually categorized into open and closed wind tunnel circuit types. Closed-circuit type wind tunnels continuously recirculate air within its closed duct work where the flow is usually driven by a large fan. Opencircuit wind tunnels contain an inlet and an outlet section where the flow is driven by inducing an overall pressure differential between the two openings. Open-circuit wind tunnels typically have an advantage over closed-circuit facilities in terms of reduced construction/operation costs and space; however, closed-circuit tunnels can achieve greater test section velocities and overall flow quality with less power.

It is also important to note the different possible driver configurations of opencircuit wind tunnels. Blowdown wind tunnels supply flow through the open-circuit using a compressor which stores pressurized air in tank reservoirs. The air is then expelled through the tunnel and out through the atmospheric outlet using a control valve which maintains constant test section flow conditions. Blower and suction type 
wind tunnels, on the other hand, make use a fan to drive the flow through the opencircuit upstream and downstream of the test section respectively. Although blowdown wind tunnels require a large amount of space to house the pressurized air reservoirs and are limited in run-times which are dependent on the overall reservoir storage capacity, these wind tunnels have the advantage of attaining large flow speeds with low test section background noise levels due to the absence of fans or motors. Suction and blower type wind tunnels are common for low-speed, low-turbulence wind tunnels, although suction type wind tunnels offer significantly better flow quality and background noise levels compared to blower configurations due to the fan being located downstream of the test section.

Figure 1.7 contains general schematics of existing wind tunnel facilities in literature; outlining some of the aforementioned different types of possible tunnel configurations that can be implemented. Figure 1.8 displays some photos of the test sections of various existing aeroacoustic wind tunnels to show how anechoic chambers are used to enclose the test sections. Finally, Table 1.1 contains a survey of relevant aeroacoustic wind tunnel facilities around the world with outlined test section and flow capabilities. Of particular interest, the ISVR aeroacoustic wind tunnel at the University of Southampton can achieve very large test section velocities (on the order of Mach 0.3) while offering one of the lowest test section background noise levels of any aeroacoustic facility in the world [35]. This is one of the main advantages of implementing blowdown type facilities, although run times can be short and test section dimensions are on the lower end of the size scale. This type of wind tunnel poses an excellent test bed for small-scale aeroacoustic research, such as TBL noise phenomena over flat plates. 


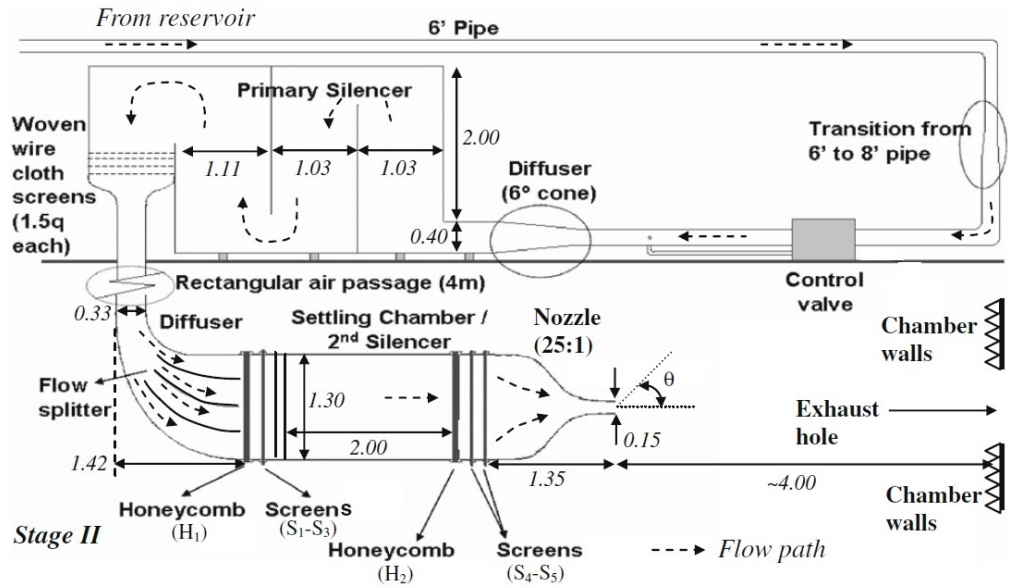

(a) ISVR Southampton open-circuit blowdown drive [36]

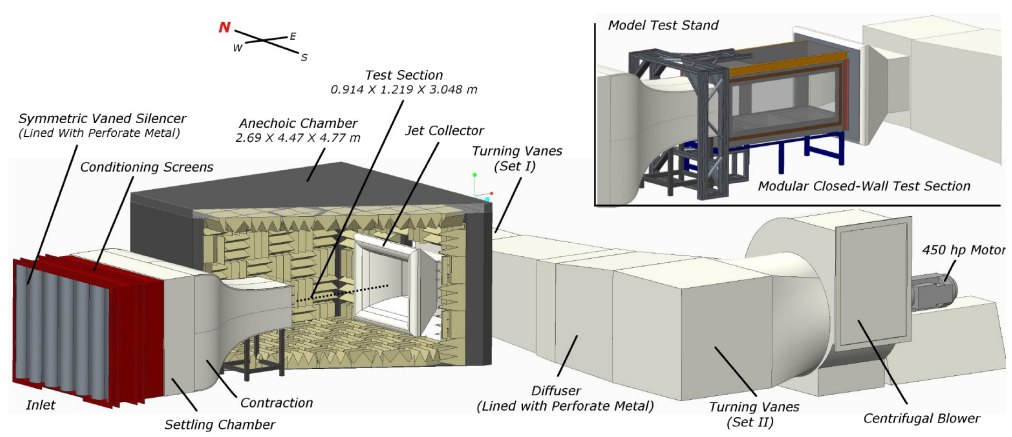

(b) Florida State University open-circuit suction drive [37]

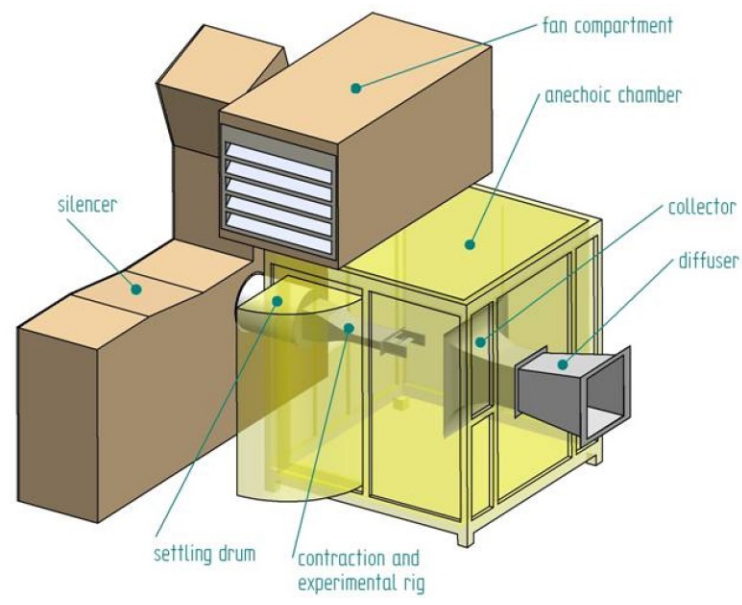

(c) University of Adelaide open-circuit blower drive 38 ]

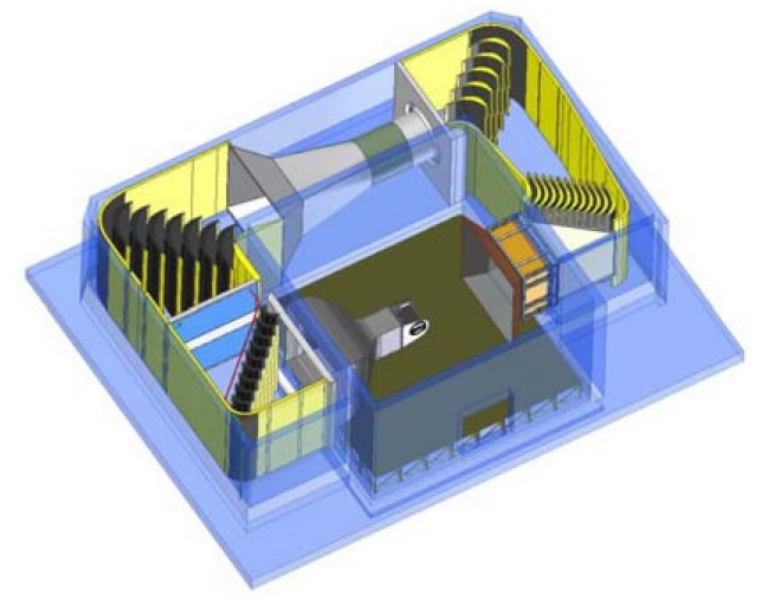

(d) DLR AWB closed-circuit fan drive [39]

Figure 1.7: Examples of anechoic wind tunnel facilities around the world - Facility schematics 


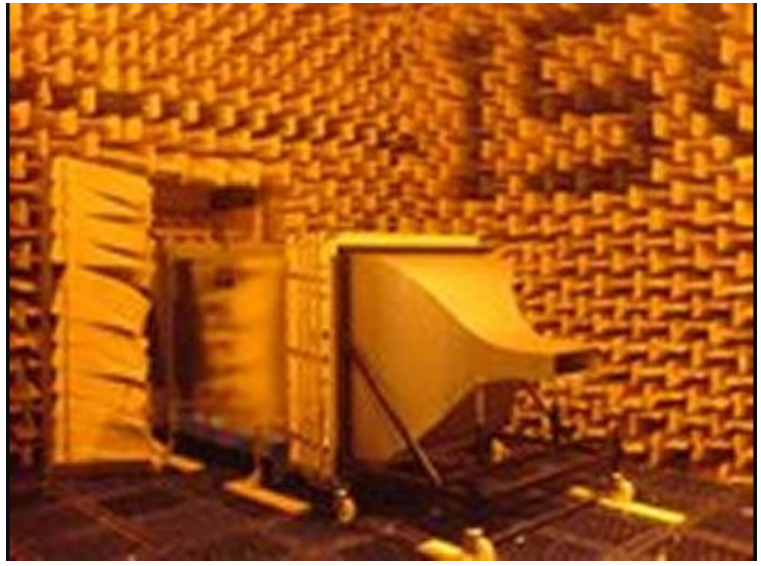

(a) ISVR Southampton 36

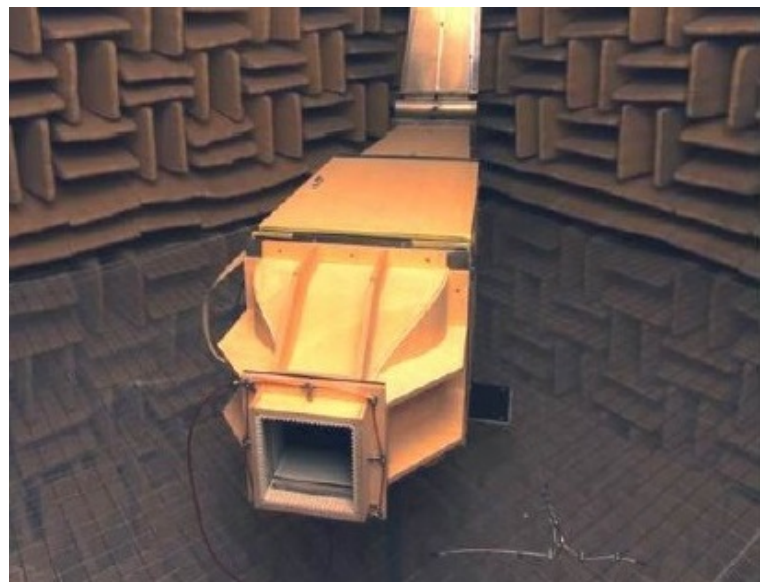

(c) Hepia-Geneve Switzerland [40]

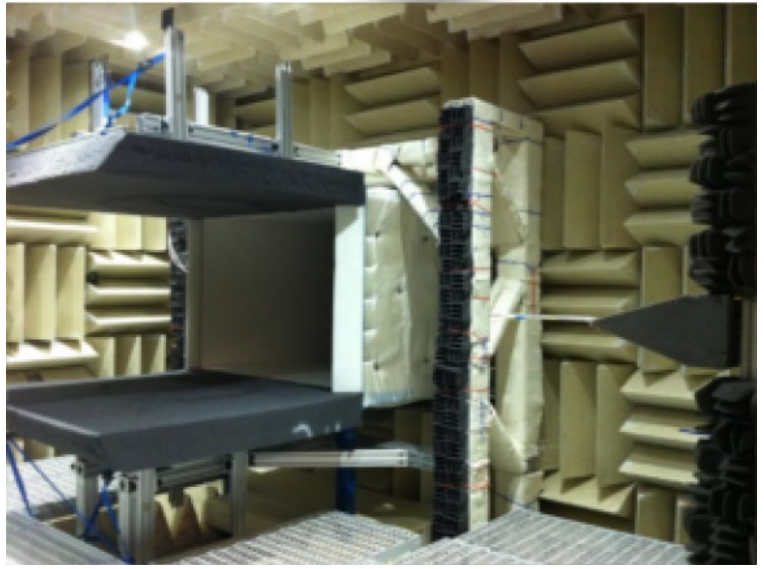

(b) Florida State University [37]

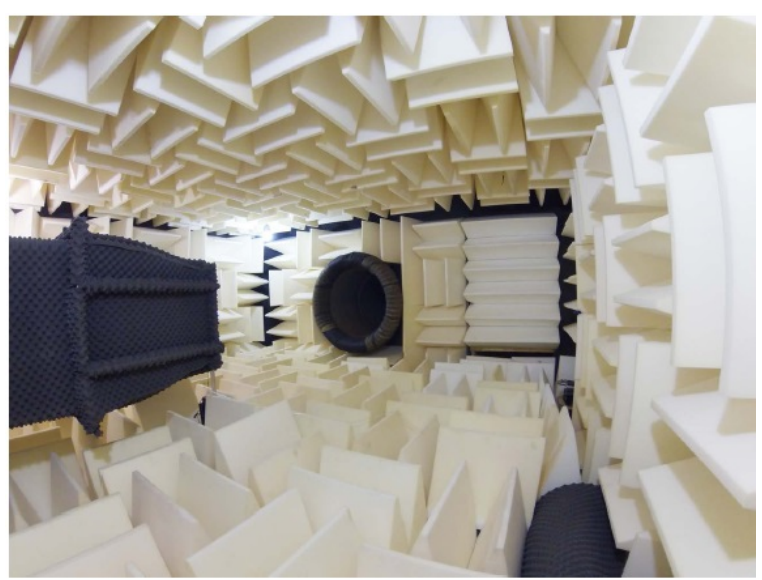

(d) UTIAS Toronto [41]

Figure 1.8: Examples of anechoic wind tunnel facilities around the world: Test section views

Since Carleton University already houses a high-speed blowdown wind tunnel, reconfiguring the facility to enable aeroacoustic testing capabilities was selected over the option of designing an entire wind tunnel facility from the ground up. The next section to follow provides details on the existing Carleton High-Speed blowdown wind tunnel (HSWT) to provide context for later discussions regarding the aeroacoustic facility design methodology and implemented fabrication methods. 
Table 1.1: Summary and survey of select aeroacoustic wind tunnel facilities around the world (data from [36 46]).

\begin{tabular}{|c|c|c|c|}
\hline Facility & Circuit Type & Test Section & Max Speed \\
\hline NASA Ames 42 & Open; Suction & $\begin{array}{c}18 m \times 9 m \\
\text { Closed }\end{array}$ & $100 \mathrm{~m} / \mathrm{s}$ \\
\hline $\begin{array}{l}\text { University of } \\
\text { Notre Dame } 43 \text {. }\end{array}$ & Open; Suction & $\begin{array}{c}0.61 m \times 0.61 m \\
\text { Open }\end{array}$ & $30 \mathrm{~m} / \mathrm{s}$ \\
\hline $\begin{array}{l}\text { University of Adelaide, } \\
\text { Australia } 38\end{array}$ & Open; Blower & $\begin{array}{c}0.225 m \times 0.075 m \\
\text { Open }\end{array}$ & $41 \mathrm{~m} / \mathrm{s}$ \\
\hline $\begin{array}{l}\text { Hepia-Geneve, } \\
\text { Switzerland [40] }\end{array}$ & Open; Blower & $\begin{array}{c}0.2 m \times 0.2 m \\
\text { Open }\end{array}$ & $20 \mathrm{~m} / \mathrm{s}$ \\
\hline University of Florida 44 & Open; Suction & $\begin{array}{c}1.12 m \times 0.74 m ; \\
\text { Open }\end{array}$ & $76 \mathrm{~m} / \mathrm{s}$ \\
\hline $\begin{array}{l}\text { Florida State } \\
\text { University } 37\end{array}$ & Open; Suction & $\begin{array}{c}1.23 m \times 0.91 m \\
\text { Open }\end{array}$ & $75 \mathrm{~m} / \mathrm{s}$ \\
\hline $\begin{array}{l}\text { Brandenburg University, } \\
\text { Germany [45] }\end{array}$ & Open; Blower & $\begin{array}{c}0.15 m \times 0.12 m ; \\
\text { Open }\end{array}$ & $72 \mathrm{~m} / \mathrm{s}$ \\
\hline $\begin{array}{l}\text { UTIAS, } \\
\text { Toronto } 41]\end{array}$ & Open; Suction & $\begin{array}{c}0.5 m \times 0.5 m \\
\text { Open }\end{array}$ & $75 \mathrm{~m} / \mathrm{s}$ \\
\hline Virgina Tech [46] & Closed; Fan & $\begin{array}{c}1.85 \mathrm{~m} \times 1.85 \mathrm{~m} ; \\
\text { Closed }\end{array}$ & $75 \mathrm{~m} / \mathrm{s}$ \\
\hline $\begin{array}{l}\text { ISVR, } \\
\text { Southampton [36] }\end{array}$ & Open; Blowdown & $\begin{array}{c}0.45 \mathrm{~m} \times 0.15 \mathrm{~m} \\
\text { Open }\end{array}$ & $100 \mathrm{~m} / \mathrm{s}$ \\
\hline $\begin{array}{l}\text { DLR AWB, } \\
\text { Germany } 39\end{array}$ & Closed; Fan & $\begin{array}{c}1.2 m \times 0.8 m \\
\text { Open }\end{array}$ & $65 \mathrm{~m} / \mathrm{s}$ \\
\hline $\begin{array}{l}\text { Carleton University } \\
\text { HSAWT, Ottawa* }\end{array}$ & Open; Blowdown & $\begin{array}{c}0.15 m \times 0.061 m \\
\text { Open }\end{array}$ & $275 \mathrm{~m} / \mathrm{s}$ \\
\hline
\end{tabular}

\footnotetext{
* Proposed wind tunnel design for comparison
} 


\subsubsection{Existing Carleton High-Speed Wind Tunnel Facility}

The HSWT at Carleton University (Figure 1.9) was selected as the base facility for the implementation of an aeroacoustic test section.

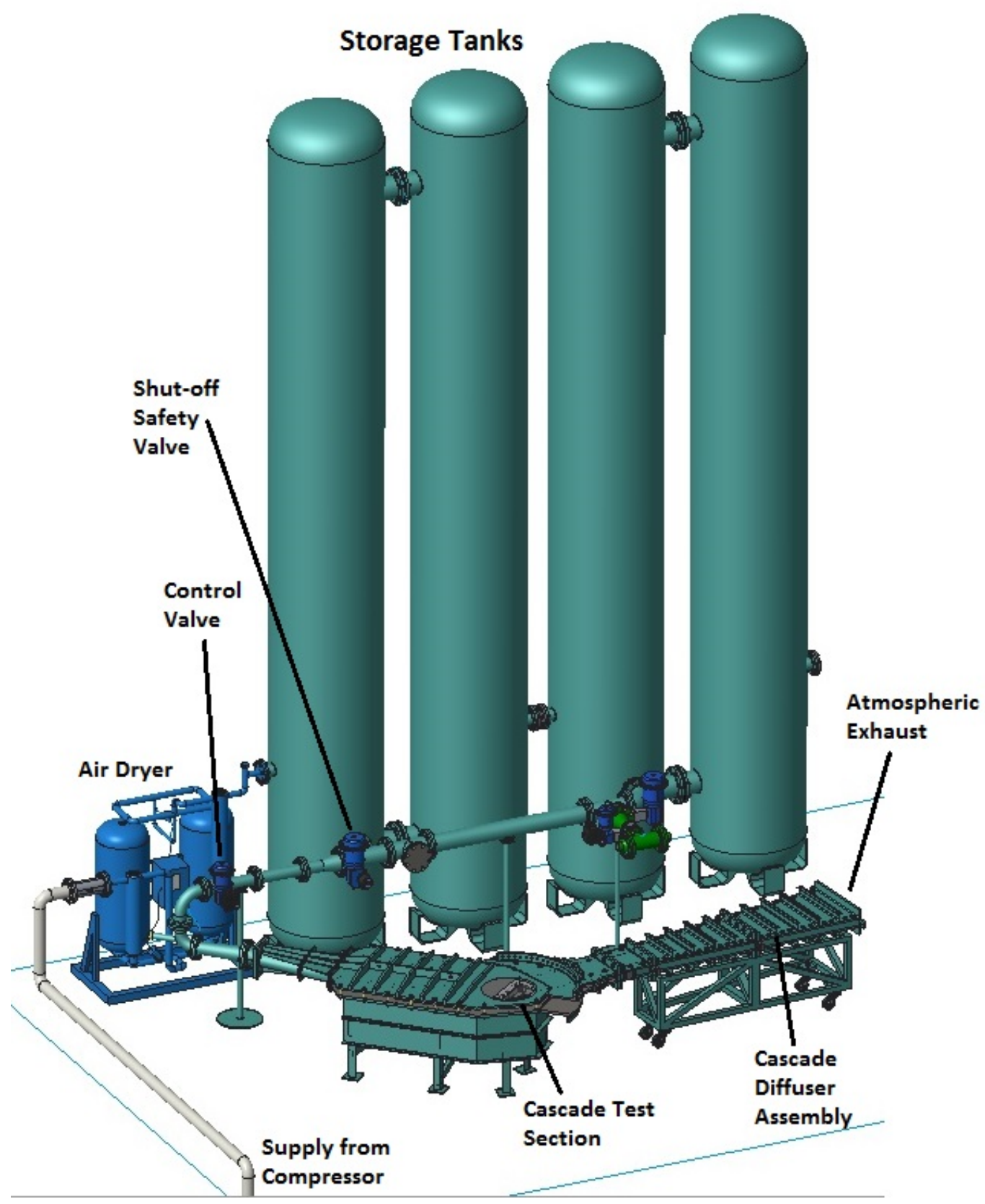

Figure 1.9: Existing Carleton HSWT facility. 
The wind tunnel is an intermittent, blowdown type facility which was originally commissioned by Jeffries [47] for the purpose of conducting transonic turbine blade row cascade research. The tunnel operates using compressed air which is delivered by a large compressor in an isolated room. The air supply is pumped through an airdyer to remove any moisture content before being supplied to four large reservoir tanks, with a total storage volume of $26 \mathrm{~m}^{3}$ and a maximum internal storage pressure of 100 psig [47]. Flow through the wind tunnel is achieved by discharging the tanks in a controlled manner through two separate valves. The first valve is a safety valve in which the operator has the ability to open or close at any time in the control room. The second valve is a control valve which is operated by a feedback controller which uses measurements of total and static pressure inside the test section to control the opening of the valve to govern constant flow conditions throughout the tunnel as the air is expelled from the tanks. The specifications of the valves are provided in Table 1.2.

Table 1.2: HSWT valve specifications [48].

\begin{tabular}{cccc}
\hline Valve & Type & Actuator & Positioner \\
\hline \hline Shut-Off & $6 "$ N.J. 815L Butterfly & N.J. QP3C-M & \\
Control & 4" N.J. 7150 Ball & N.J. QP2C-M & NE724 Electro-Pneumatic \\
\hline
\end{tabular}

The design strategy for reconfiguring the HSWT for the implementation of an aeroacoustic test chamber is to make use of as much of the existing facility components as possible to minimize cost. One restriction in doing so however involves making no major modifications to the current equipment so as to not disturb or interrupt present and future blade row cascade research. Figure 1.10 contains a detailed view of the section of the wind tunnel with the control valve and piping leading to the cascade test section. The removable 90 degree bent pipe just downstream of the control valve 
would allow for relatively quick attachment and detachment of a secondary system for delivering flow (such as a flexible high pressure air hose) to the aeroacoustic test chamber that is positioned accordingly in the facility away from other current equipment. This implementation strategy would allow for flexibility in the placement of the HSAWT equipment in the facility while ensuring that the cascade test section flow path within the wind tunnel can be used with minimal downtime in switching between the two configurations. Making use of both wind tunnel valves, as well as the current flow control system, ensures that implementation costs are significantly lowered.

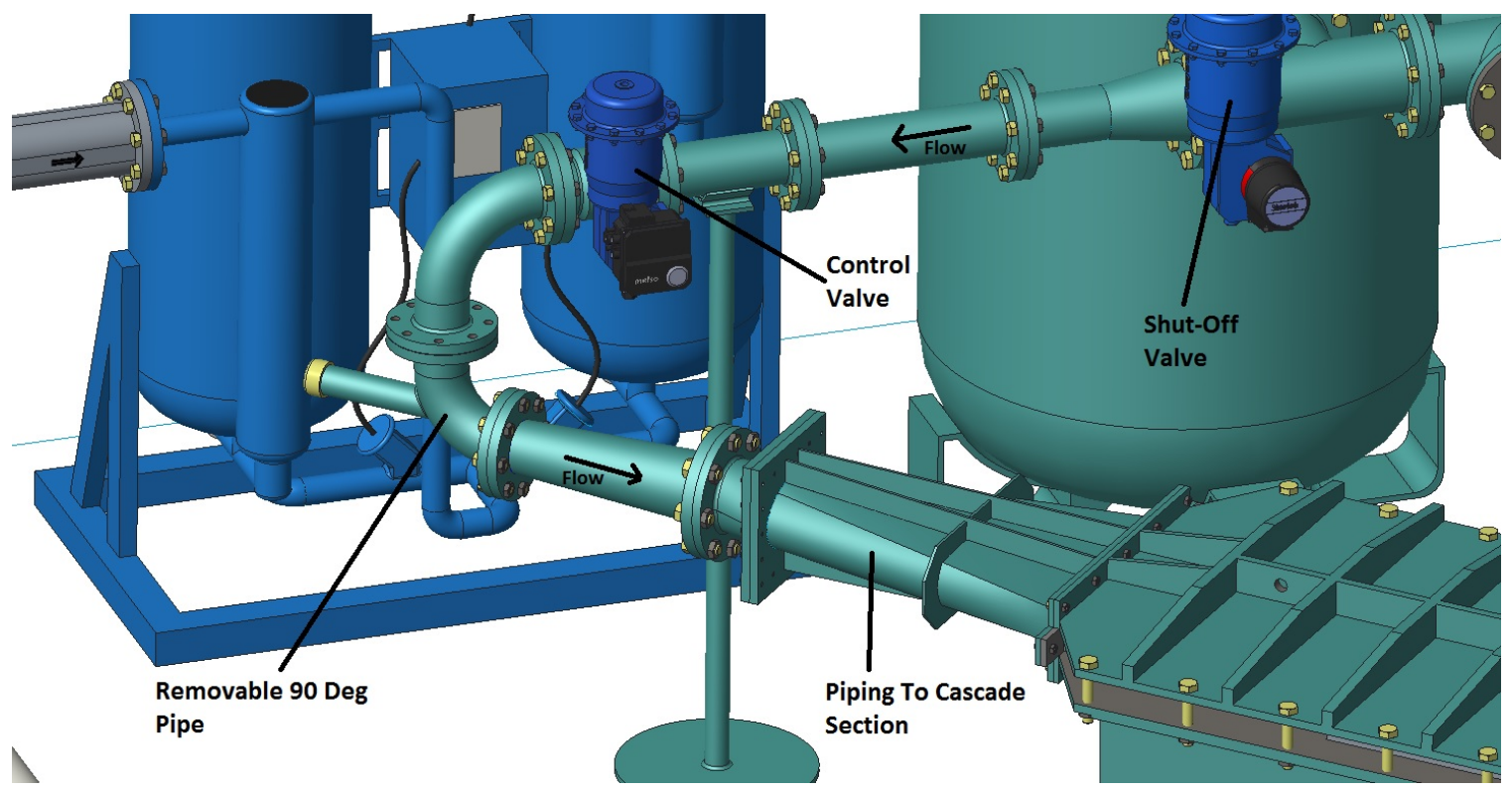

Figure 1.10: Detailed View of Control Valve Upstream Piping.

In addition to using a flexible high pressure air hose to deliver a standalone flow path to the test chamber, a secondary piece of equipment that was previously unused in the facility was selected as the main interface between the hose and the chamber. This straight channel assembly (Figure 1.11) is constructed of thick steel walls and is mounted on a cart with wheels, thus allowing for movement and storage when not 
in use. The dimensions of the internal flow channel are $6.1 \mathrm{~cm} \times 30 \mathrm{~cm}$ and is 1.524 $m$ in length. The use of this channel essentially removes the need to manufacture additional flow piping with significant length in order to reach the proposed position of the test chamber.

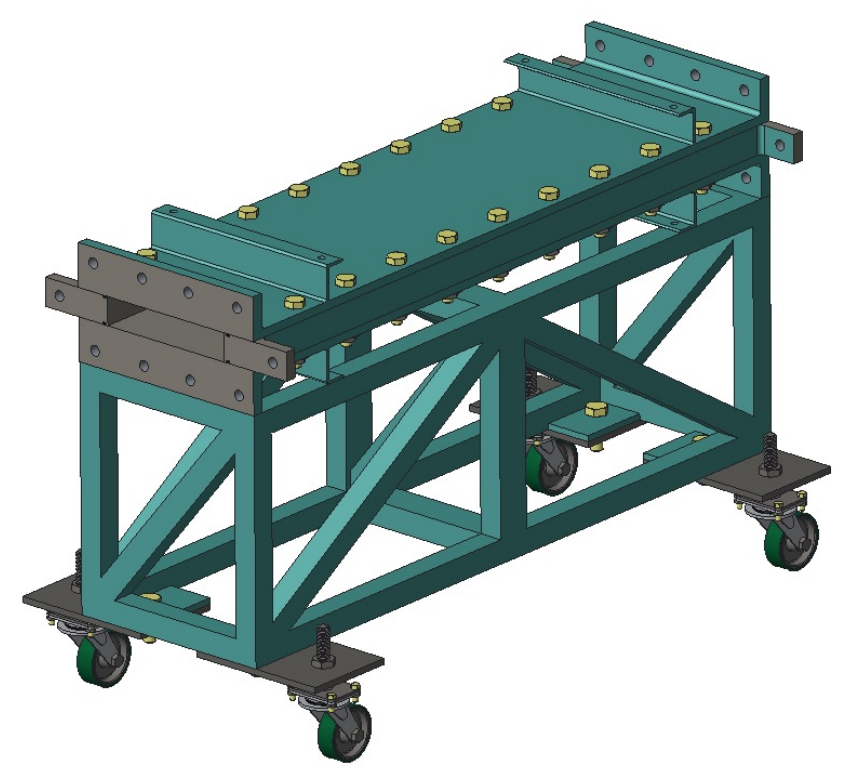

Figure 1.11: Straight channel assembly.

\subsection{Research and Thesis Objectives}

The overall purpose of this thesis is to expand Carleton's capabilities in examining TBL surface pressure fluctuation behavior at various high speed flow conditions. This entails the following key objectives:

1. Develop a unique high-speed aeroacoustic wind tunnel, using an existing blowdown facility, capable of conducting acoustic measurements of subjects exposed to high speed flow with low background noise;

2. Perform the complete design and fabrication work for the wind tunnel; 
3. Commission the wind tunnel for testing, including the development of the instrumentation and wind tunnel flow control systems;

4. Perform preliminary measurements of the single-point surface pressure fluctuation spectrum at various flow speeds (low to high subsonic range) and compare to semi-empirical models developed in literature;

5. Determine recommendations for future facility characterization work and improvements.

\subsection{Thesis Structure}

The forthcoming chapters of the thesis are structured in the following manner:

- Chapter 2 - provides details regarding the complete design methodology implemented for the individual HSAWT components;

- Chapter 3 - describes the methods used in the fabrication and installation of the designed wind tunnel components;

- Chapter 4 - details the experimental methods used for wind tunnel operation and data collection, including all instrumentation and control system hardware;

- Chapter 5 - presents sensor and flow speed calibration data, along with flow characterization results from initial commissioning;

- Chapter 6- outlines preliminary results of single-point surface pressure fluctuation spectrum measurements conducted at various flow speeds;

- Chapter 7 - summarizes with key conclusions and recommendations for future experimental studies and possible improvements to the facility. 


\section{Chapter 2}

\section{Aeroacoustic Wind Tunnel Design Methodology}

This chapter details the entire design methodology behind each new HSAWT component. This includes descriptions of newly fabricated components and how they interface with some of the existing equipment in the facility. First a list of overall design criteria is presented, followed by a summary of the entire facility layout. The preliminary conducted flow analysis and wind tunnel feasibility study is then outlined. Finally, the acoustic and aerodynamic design parameters for each component are described in detail.

\section{$2.1 \quad$ Design Requirements}

The overall purpose of the implementation of the aeroacoustic wind tunnel is to facilitate flat plate TBL pressure fluctuation studies in a high speed environment that mimics flow conditions seen by real aircraft. For this reason, the main requirement for the facility is to obtain Mach number similitude with general business and commercial aircraft. An upper range of Mach 0.75 - 0.8 was selected as the target test section freestream speed to obtain. In terms of Reynolds number similarity, boundary layer 
studies usually involve the momentum thickness as the characteristic length scale. This parameter is difficult to measure on aircraft and usually involves the use of analytical TBL theory equations to estimate this parameter with flat plate development assumptions. Reynolds number similarity is therefore a secondary requirement, where a wind tunnel with easy access to the test section upstream components (flow conditioning equipment, settling chamber, nozzle, etc.) can allow for TBL modification devices to be installed (such as a boundary layer trip system) to attempt to modify the boundary layer properties to match the conditions estimated by aircraft measurements. A fundamental requirement however is that the boundary layer in the test section is fully developed over a Zero Mean-Stream Pressure Gradient (ZMPG). Other test section mean stream flow requirements based on aggregate data from other wind tunnel facilities in literature include achieving flow non-uniformity below $1 \%$ and a Turbulence Intensity (TI) below $1 \%$.

Another important factor to account for in the use of a blowdown configuration wind tunnel is the allowable run time before the supply from the reservoir tanks is depleted. The usable run time for the wind tunnel is dictated by the test section speed, pressure ratio, and required mass flow rate, $\dot{m}$, needed to overcome the total head losses in the wind tunnel. A run time of at least 30 seconds was selected as a target parameter; ensuring that statistical convergence on measured pressure fluctuation data is obtained. This target is based loosely on previous measurements conducted within Carleton's MSAWT facility. The test section must also be able to house the removable TBL test panel that was developed for the MSAWT test set-up.

In terms of the acoustic requirements of the test section, the expected experimental measurements must achieve a Signal-to-Noise Ratio (SNR) larger than $10 d B$ relative to chamber background noise levels across all frequency bands of interest to resolve the proper spectral behavior. A chamber cut-off frequency that is as low as possible 
is also beneficial to obtain measurements that resolve the low frequency region of the spectrum. Both of these requirements involve implementing an anechoic chamber with walls and wedges as large as possible. Obviously spatial and cost limitations pose an upper limit, therefore the target size of the chamber was selected to be as large as the facility would allow. A target cut-off frequency of $250 \mathrm{~Hz}$ was chosen in relation to a reasonable acoustic chamber wedge size. Some alternative restrictions imposed on the design include an ability to move the test chamber around the facility so that it can be stored when not in use, as well as including provisions for adequate exhaust of the flow out of the chamber and into the facility. This is to ensure that the flow exhaust does not damage the chamber internally as well as any surrounding research equipment.

Table 2.1 contains a summary of the major design requirements discussed above.

Table 2.1: Summary of major HSAWT design requirements.

\begin{tabular}{|l|c|l|}
\hline Requirement & Target & Comments \\
\hline \hline $\begin{array}{l}\text { Max Test Section } \\
\text { Mach Number }\end{array}$ & $0.75 \leq M \leq 0.8$ & $\begin{array}{l}\text { General cruise } \\
\text { flight speeds }\end{array}$ \\
\hline $\begin{array}{l}\text { Min Wind Tunnel } \\
\text { Run Time }\end{array}$ & $30 \mathrm{~s}$ & $\begin{array}{l}\text { Sufficient run time for } \\
\text { data collection }\end{array}$ \\
\hline $\begin{array}{l}\text { Test Section } \\
\text { Flow Quality }\end{array}$ & Flow non-uniformity: $<1 \%$ & Similar wind tunnel \\
Test Chamber & Cut-off frequency: $<250 \mathrm{~Hz} z$ & Similar wind tunnel \\
Acoustic Quality & Measurement SNR: $>10 \mathrm{~dB}$ & facility criteria \\
\hline $\begin{array}{l}\text { Other } \\
\text { requirements }\end{array}$ & $\begin{array}{c}\text { Movable anechoic test chamber } \\
\text { Adequate exhaust of flow }\end{array}$ & $\begin{array}{l}\text { Based on departmental } \\
\text { requirements }\end{array}$ \\
\hline
\end{tabular}




\subsection{Aeroacoustic Wind Tunnel Facility Layout}

The complete Carleton HSAWT facility configuration is displayed in Figure 2.1. with alternate views of the facility contained in Figure 2.2

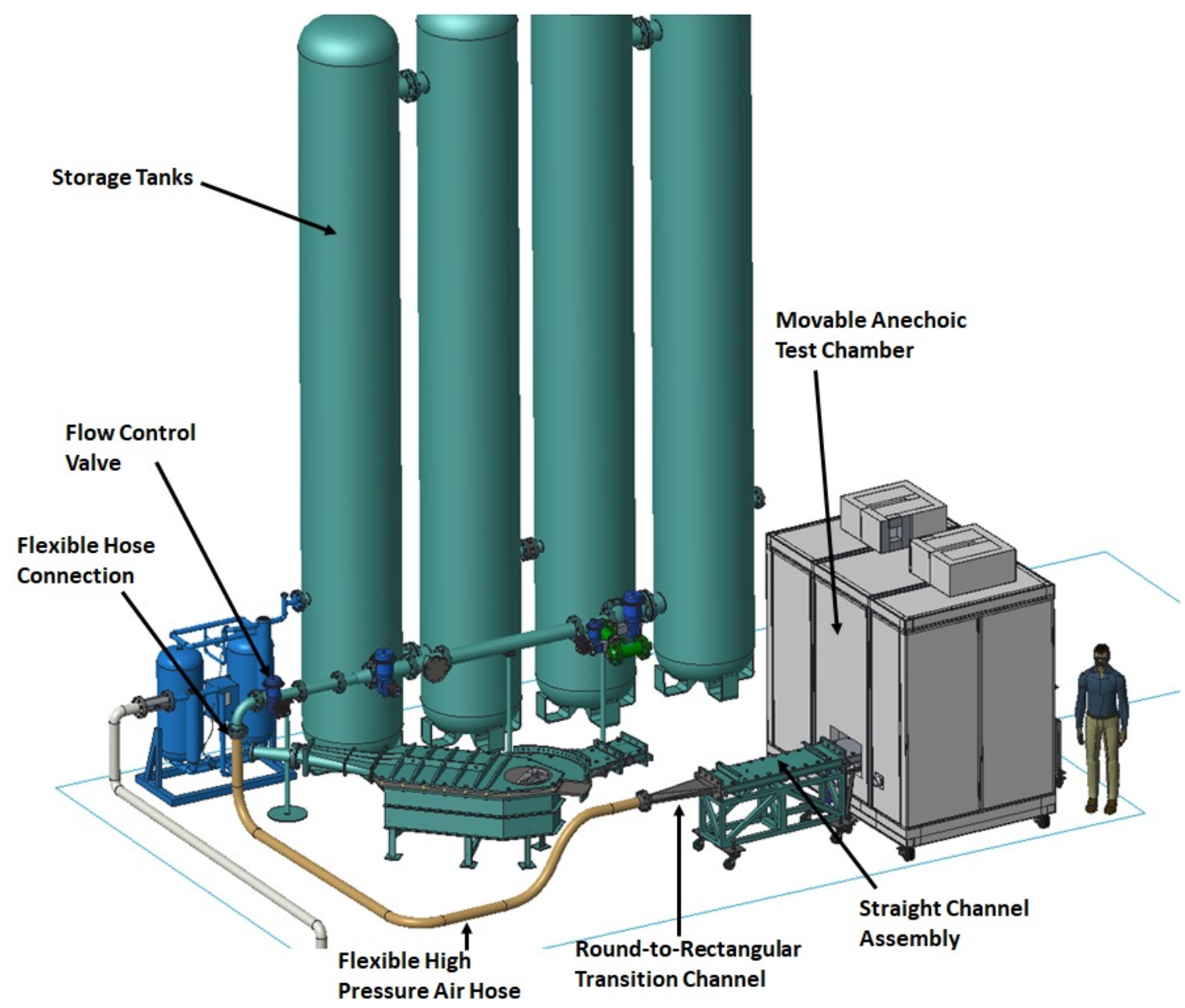

Figure 2.1: Complete Carleton HSAWT facility.

The aeroacoustic configuration of the high-speed facility makes use of a fully anechoic chamber to house the open jet test section. The chamber is placed in a specific area within the facility to provide adequate space to manoeuvre around the room. The anechoic chamber is built with 4" thick acoustic paneled walls and is lined 


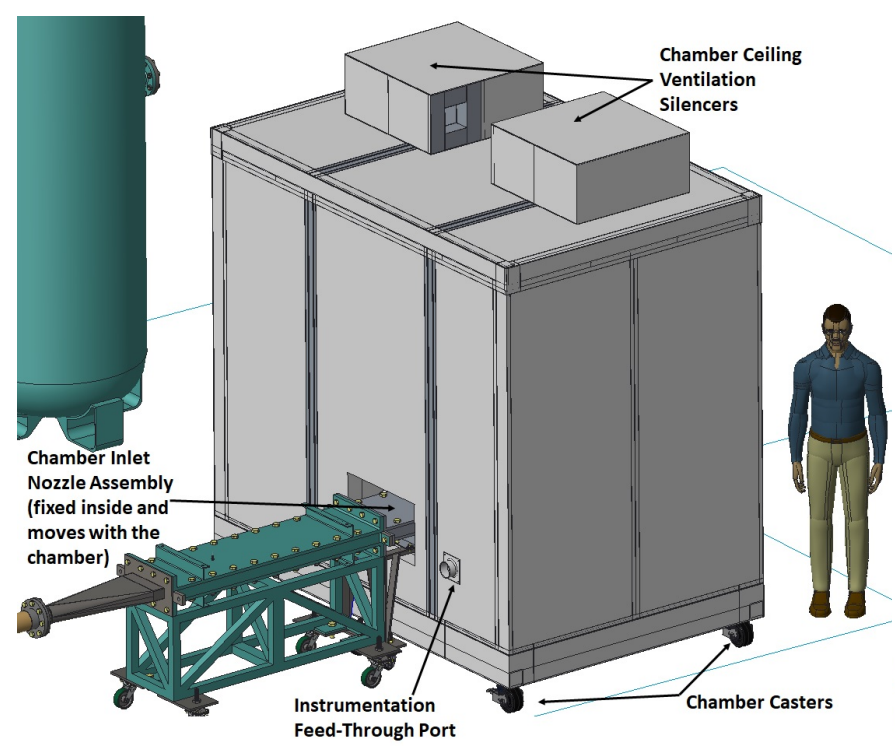

(a) Chamber Front View

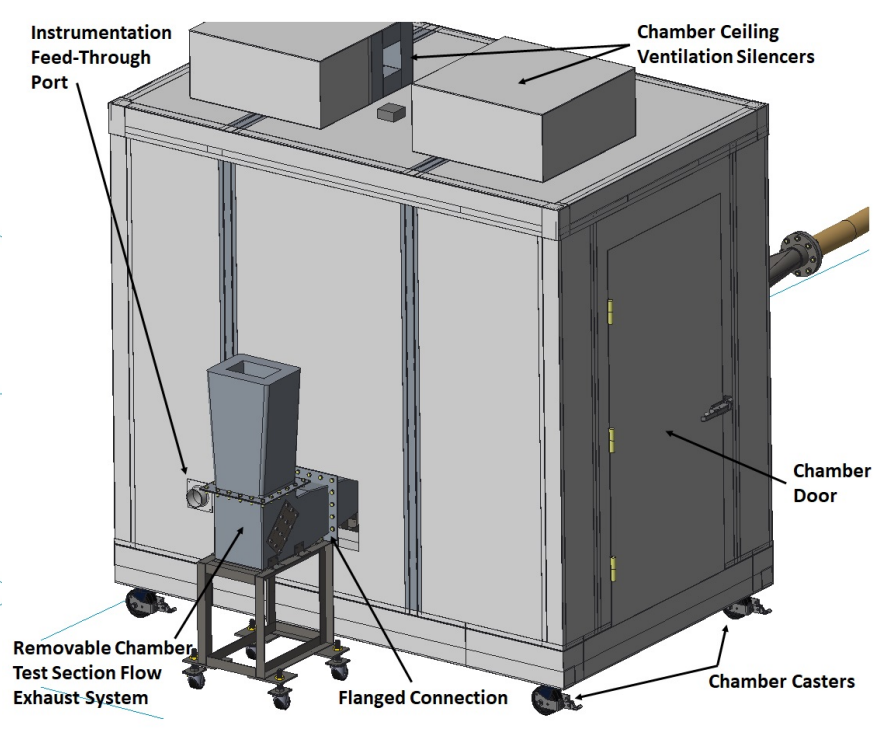

(b) Chamber Rear View

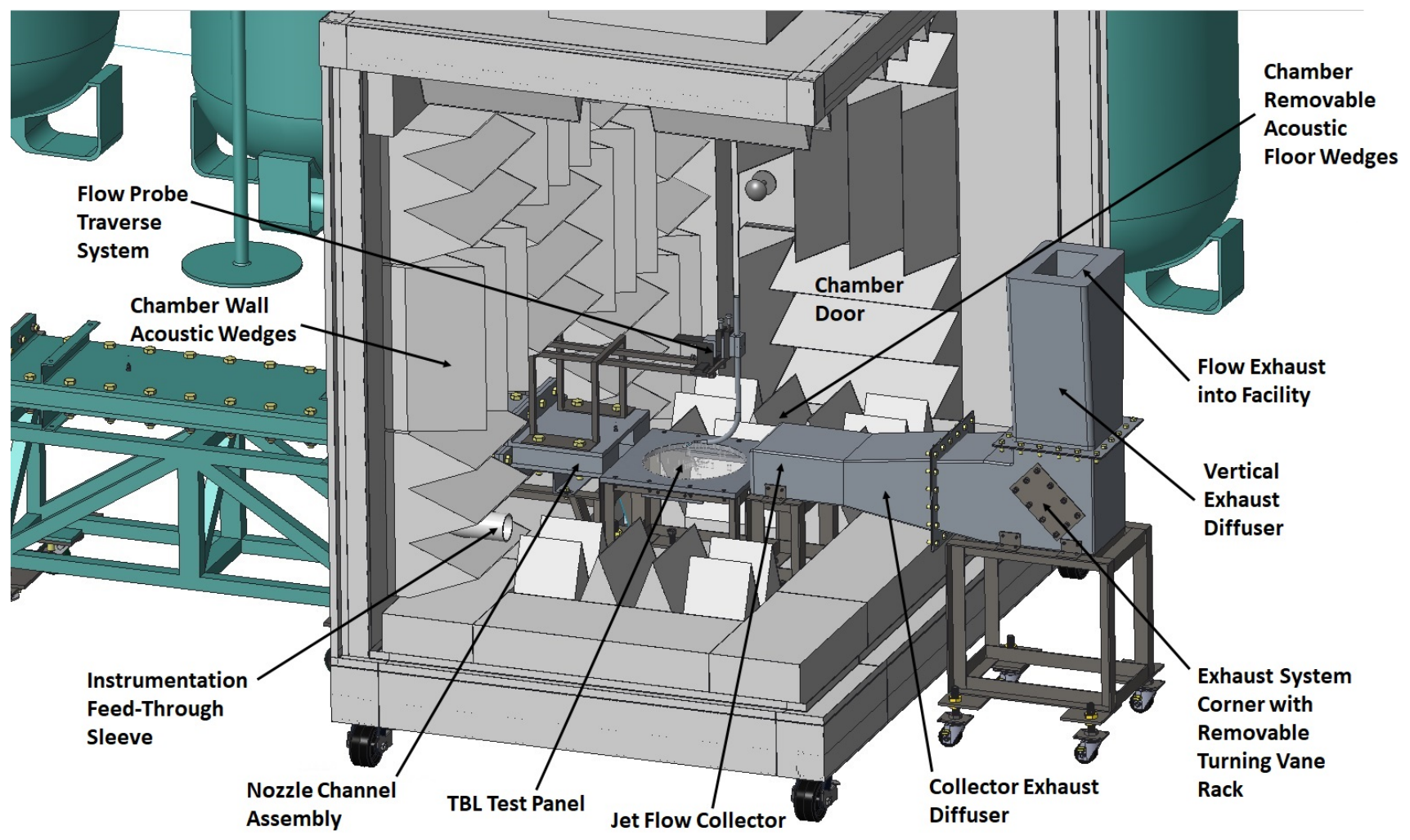

(c) Chamber Interior View

Figure 2.2: Carleton HSAWT alternate facility views. 
with perforated sheet metal acoustic wedges along all interior surfaces; providing the chamber with a sound attenuation cut-off frequency of $250 \mathrm{~Hz}$. The floor wedges are designed to be removable, which allows for researchers to gain access to all areas inside of the chamber. The chamber also contains two rectangular cut-out holes on the upstream and downstream walls so that the wind tunnel duct components can be fed through to the inside of the chamber. Two ventilation holes on the ceiling of the chamber allow for pressure equalization to ensure that the chamber walls are not loaded in an unsafe manner when the tunnel is in operation at high speeds. These holes are fitted with ventilation silencers to ensure that facility background noise travelling into the chamber is attenuated.

When the HSAWT configuration is in place, the original cascade diffuser channel is removed and stored off to the side of the facility. The $90^{\circ}$ bent pipe is removed just downstream of the control valve (refer to Figure 1.10) and a flexible high pressure air hose is connected in its place to deliver a flow path to the chamber. The hose is then connected to a newly designed round-to-rectangular transition channel, which diffuses the flow slightly and provides a smooth transition from the 4" round hose to the rectangular cross-section of the existing straight channel assembly. The transition duct is designed to house a honeycomb flow straighter device near its outlet, with a cell size of $3.175 \mathrm{~mm}$ and a cell depth of $1.59 \mathrm{~cm}$, which is used to ensure that the flow is uniform entering the straight channel assembly. An additional flow conditioning screen with an open area ratio, $\sigma=0.65$, is also used in between the transition duct and straight channel, fixed in between the flange connections, to further condition the flow entering the channel. The purpose of using the straight channel assembly is therefore to act as a settling duct to help dissipate small-scale turbulence in the flow after it has been passed through the inlet flow conditioners. 
Flow from the straight channel duct is passed through an additional flow conditioning screen $(\sigma=0.59)$, before entering a newly fabricated nozzle channel assembly. The nozzle channel assembly first contains a straight section, which combined provides a total settling duct length of $2.05 \mathrm{~m}$. The flow then feeds into the nozzle section which is designed with contoured side walls that accelerate the flow to the required conditions inside the test section. The nozzle section length is $35.56 \mathrm{~cm}$ and accelerates the flow with an outlet to inlet area contraction ratio, $C R$, of 2 . The nozzle outlet dimensions are therefore $15 \mathrm{~cm} \times 6.1 \mathrm{~cm}$. The entire nozzle assembly passes through the chamber inlet clearance hole and into the test section. The remaining open area between the nozzle channel and the clearance hole is sealed off with acoustic insulation. The nozzle channel is also fixed on top of a support structure which is fixed internally to the chamber and externally on casters. This allows the for the nozzle channel to protrude slightly outside of the chamber to connect to the straight channel assembly when the chamber is in use; while also allowing for the entire system to stay fixed to the chamber when it is disconnected and moved to its storage position should the original wind tunnel configuration be implemented. Figure 2.3 contains a detailed view of the nozzle component, its support structure, and how it interfaces with the straight channel duct.

Figure 2.4 contains a detailed view of the open jet test section inside the chamber, which is $0.508 \mathrm{~m}$ in length. For the purpose of conducting TBL surface pressure fluctuation studies, the test section is equipped with a support structure which houses a previously designed circular acrylic test panel which is used in similar low speed research within the MSAWT facility at Carleton University [17]. The panel is designed to rotate, providing high spatial resolution accuracy for the flush mounted pressure fluctuation sensors, and to sit flush with the floor of the nozzle outlet orifice so that 


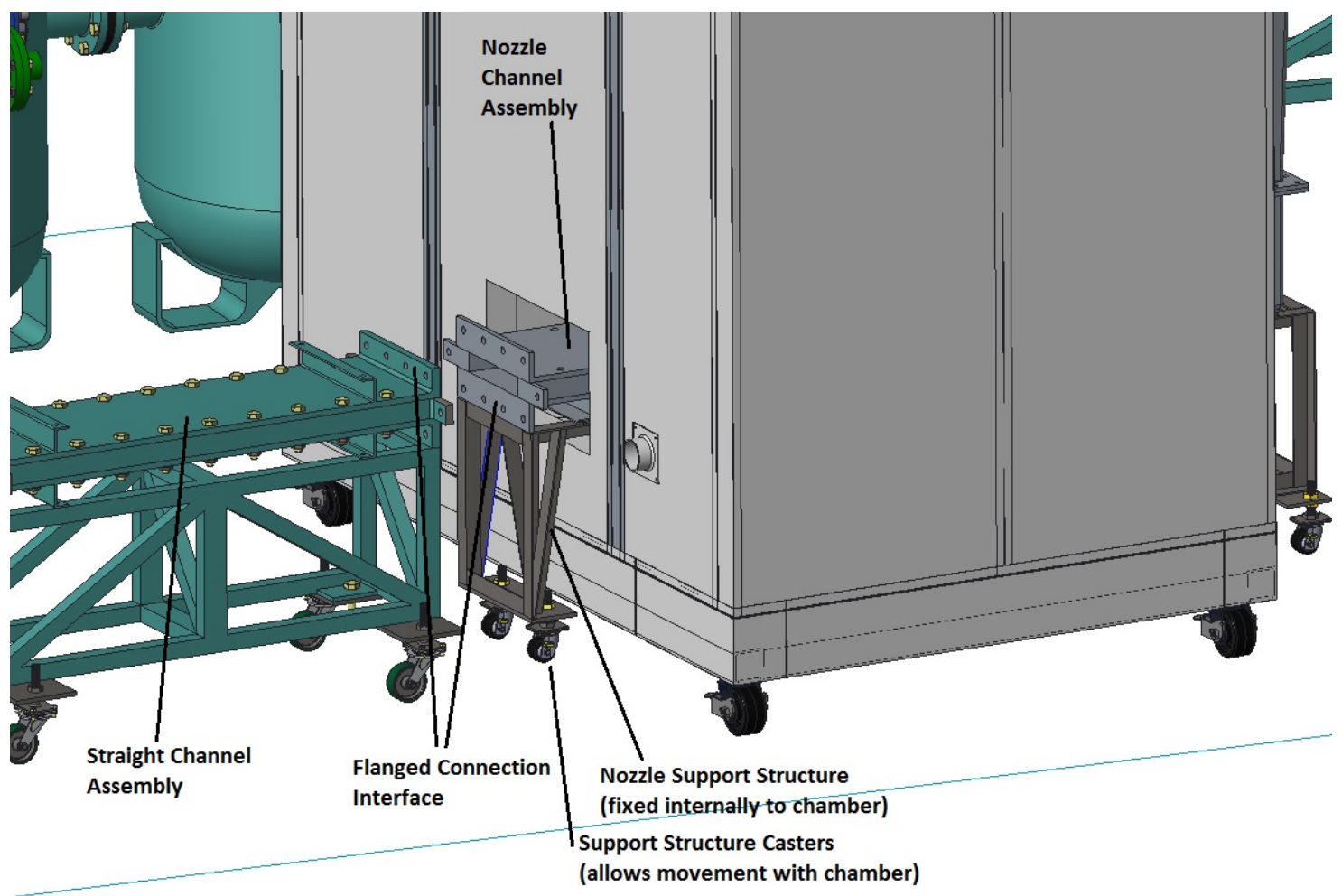

Figure 2.3: Nozzle channel assembly connection interface.

the development of the boundary layer is minimally disturbed. The panel and support structure can also be removed to facilitate other aeroacoustic test section configurations. A support structure, which is fixed to the top of the nozzle channel assembly, houses the removable traverse system and flow probe instrumentation holder that was designed for the MSAWT set-up [17]. This instrumentation probe traverse system can house a variety of flow measurement devices such as a hot-wire or pitot-static probe which can be used to characterize mean flow and boundary layer properties.

Flow from the nozzle orifice is exhausted out from the chamber using a two-piece flow exhaust system. The first piece is fed through the chamber outlet clearance hole and remains fixed inside the chamber to an internal support, with a connection flange that remains on the exterior side of the chamber outlet wall. The second piece of the exhaust system is mounted on a stand-alone support cart with casters, allowing for it 


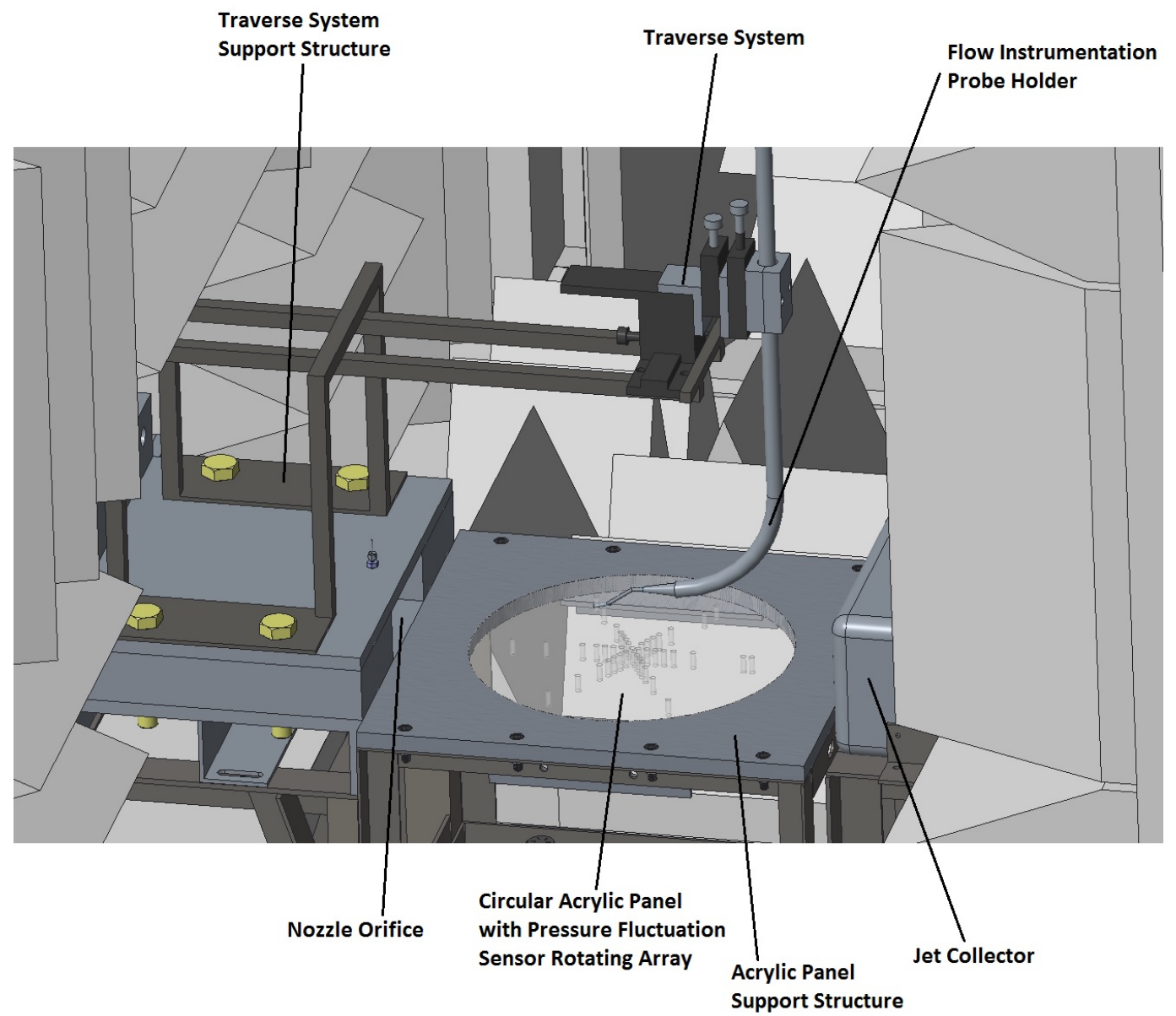

Figure 2.4: Nozzle channel assembly connection interface.

to be connected to the fixed part of the exhaust system by a bolted flange connection when in use, and to be stored away when not in use. The internal flow components of the exhaust system first consists of a jet collector with smooth inlet mouth dimensions of $21.2 \mathrm{~cm}$ by $9.4 \mathrm{~cm}$; which is used to capture both the core and shear layer of the jet to minimize the development of a longitudinal static pressure gradient inside the test section. The collector then contracts the flow to an internal cross-section of 15.24 $\mathrm{cm}$ by $7.62 \mathrm{~cm}$ to minimize flow separation and non-uniformity before being fed into the collector exhaust diffuser. This diffuser is $0.56 \mathrm{~m}$ in length and provides some pressure recovery for the wind tunnel with an outlet to inlet area ratio, $A R$, of 2 and 
a total wall divergence angle, $\theta_{\text {diff }}$, of $7.7^{\circ}$. Flow from the first diffuser is then passed through a vertical $90^{\circ}$ corner, fitted with a removable flow turning vane rack assembly to ensure minimal flow separation along the corner. The turning vane rack assembly consists of 4 circular-arc thin sheet metal turning vanes with trailing edge extensions, each with a total chord, $c$, of $0.0824 \mathrm{~m}$; a constant thickness-to-chord ratio, $t / c$, of 0.0433; and a vane spacing-to-chord ratio, $h / c$, of 0.485 . Finally, the corner is attached to a second vertical diffuser channel to exhaust the flow out to atmosphere inside the facility. The vertical diffuser provides further pressure recovery with a length of 0.61 $m, A R$ of 1.56 , and $\theta_{\text {diff }}$ of $8^{\circ}$.

Vertical exhaust of the flow is required due to the close proximity of the interior facility walls with respect to the outlet wall of the test chamber. All of the interior duct walls of the removable section of the exhaust system are acoustically treated with bulk acoustic insulation enclosed by fiber glass cloth and perforated sheet metal to help attenuate noise propagation. A secondary flange connection between the vertical exhaust diffuser and corner allows for internal access to the corner channel and turning vane assembly for maintenance purposes.

Figure 2.5 contains a labeled plan view of the entire facility to show the placement of the aeroacoustic wind tunnel components. Figure 2.6 contains labeled section views of the chamber to clarify the details of the components throughout the wind tunnel. Finally, Table 2.2 contains a complete summary of the design aspects of the individual HSAWT components. The sub-sections to follow in this chapter outline details of the preliminary flow analysis conducted to assure the feasibility of the implemented duct configuration, as well as the detailed design methodology behind each of these components. 


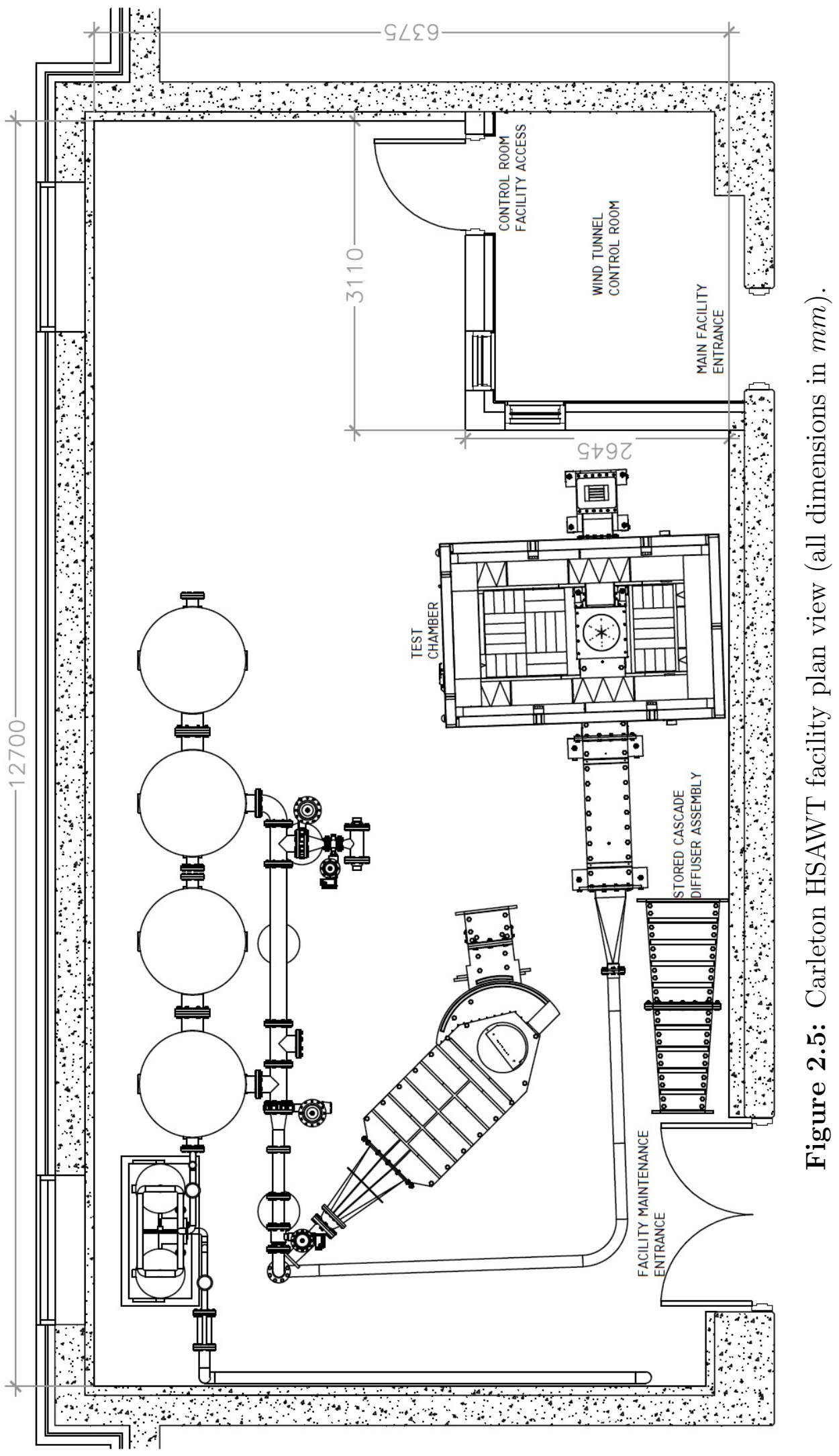




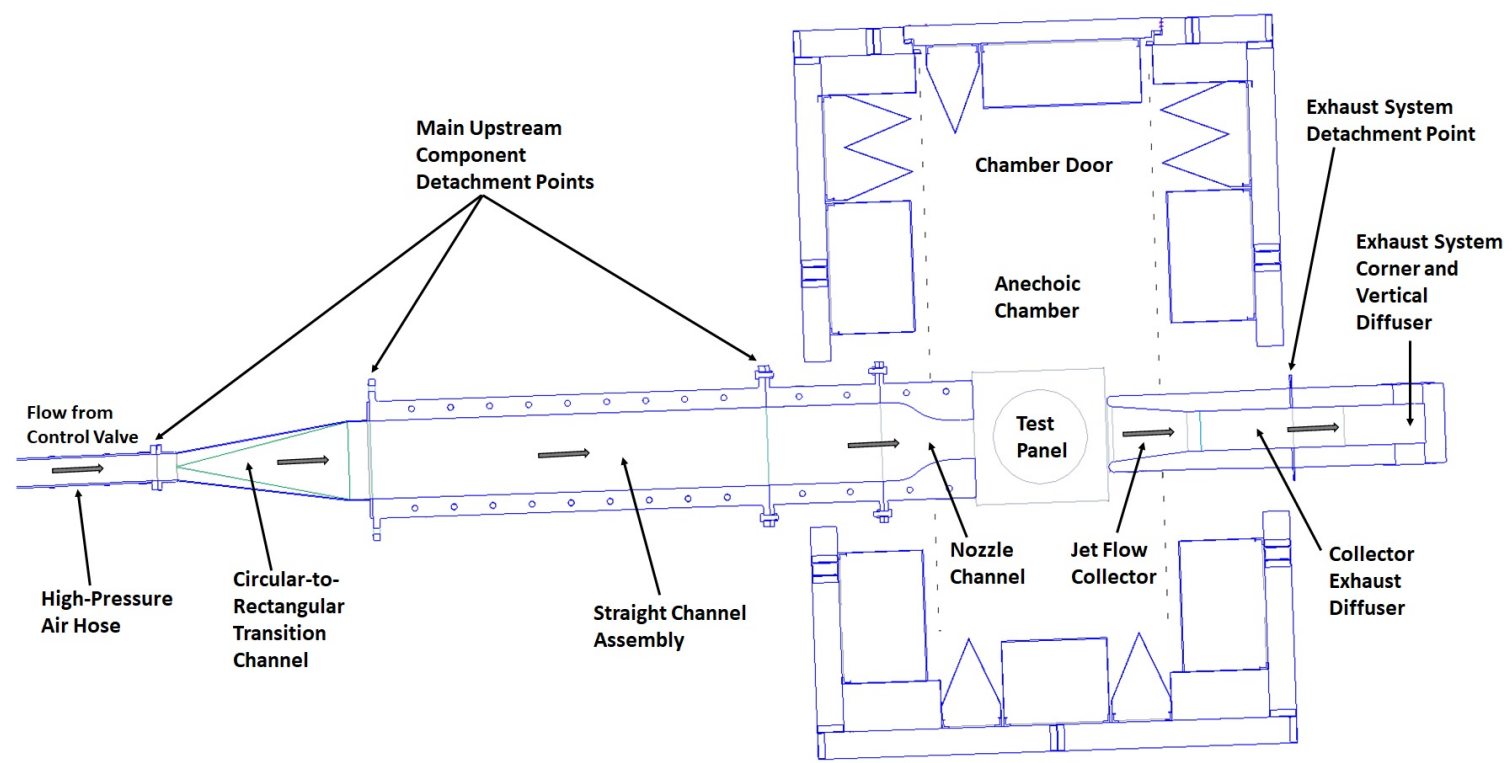

(a) Section Plan View

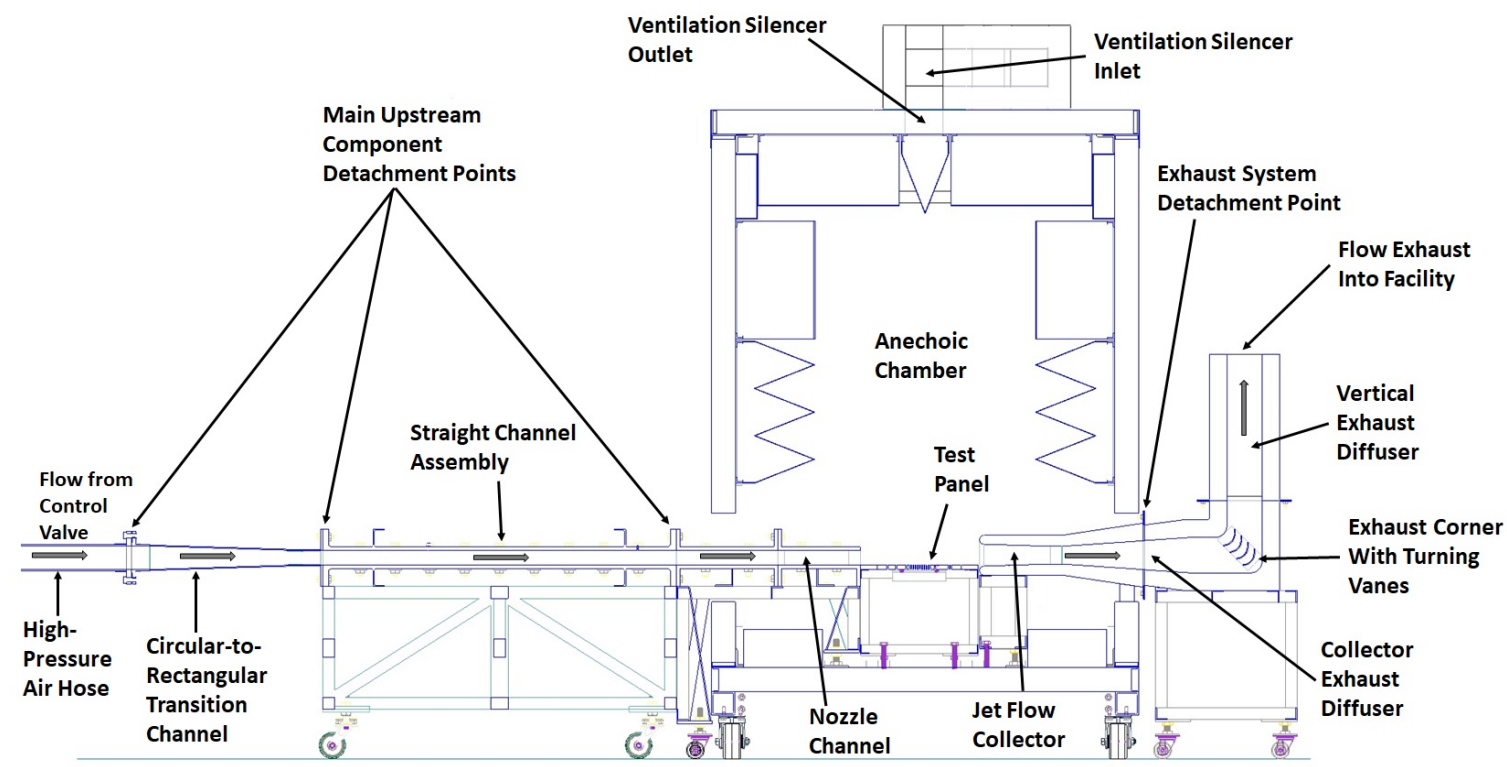

(b) Section Elevation View

Figure 2.6: Detailed section views of the HSAWT facility. 
Table 2.2: Summary of HSAWT major component design parameters.

\begin{tabular}{|c|c|c|}
\hline Component & Dimensions & Comments \\
\hline High Pressure & Inner diameter: $10.16 \mathrm{~cm}$ & 150 psi \\
\hline Flexible Hose & Total length: $7.62 \mathrm{~m}$ & working pressure \\
\hline Round-to- & Inlet: $10.16 \mathrm{~cm}$ dia. & Honeycomb conditioner: \\
\hline Rectangular & Outlet: $30 \times 6.1 \mathrm{~cm}$ & Cell size: $3.175 \mathrm{~mm}$ \\
\hline Transition Duct & Length: $0.66 \mathrm{~m}$ & Cell length: $1.59 \mathrm{~cm}$ \\
\hline Settling Duct & Total length: $2.05 \mathrm{~m}$ & Screen $\# 1: \sigma=0.65$ \\
\hline (Flow Conditioners) & Channel: $30 \times 6.1 \mathrm{~cm}$ & Screen $\# 2: \sigma=0.59$ \\
\hline Nozzle Section & $\begin{array}{c}\text { Section length: } 35.56 \mathrm{~cm} \\
\text { Outlet: } 15 \times 6.1 \mathrm{~cm}\end{array}$ & $\begin{array}{l}C R=2 ; 3 \text { rd-order } \\
\text { polynomial profile }\end{array}$ \\
\hline Test Section & Open-jet length: $0.508 \mathrm{~m}$ & $\begin{array}{l}\text { Open jet with } \\
\text { flush test panel }\end{array}$ \\
\hline $\begin{array}{l}\text { Anechoic Test } \\
\text { Chamber }\end{array}$ & $\begin{array}{l}\text { Outer: } 2.8 \times 1.842 \mathrm{~m} \\
\text { Inner: } 1.907 \times 0.883 \mathrm{~m} \\
\text { Total height: } 2.796 \mathrm{~m} \\
\text { Internal height: } 1.95 \mathrm{~m}\end{array}$ & $\begin{array}{l}\text { Movable with casters, } \\
\text { Perforated metal wedges, } \\
250 \mathrm{~Hz} \text { cut-off frequency, } \\
\text { Wedge height: } 0.343 \mathrm{~m}\end{array}$ \\
\hline Jet Collector & $\begin{aligned} \text { Inlet: } 21.2 \times 9.4 \mathrm{~cm} \\
\text { Outlet: } 15.24 \times 7.62 \mathrm{~cm}\end{aligned}$ & $\begin{array}{l}\text { Acoustically treated lips; } \\
3.8 \mathrm{~cm} \text { lip diameter }\end{array}$ \\
\hline $\begin{array}{l}\text { Collector } \\
\text { Exhaust } \\
\text { Diffuser }\end{array}$ & $\begin{array}{c}\text { Length: } 0.56 \mathrm{~m} \\
\text { Outlet: } 15.24 \times 15.24 \mathrm{~cm} \\
\theta_{\text {diff }}=7.7^{\circ}\end{array}$ & $\begin{array}{l}\mathrm{AR}=2 ; \text { Acoustically } \\
\text { treated walls }\end{array}$ \\
\hline $\begin{array}{l}\text { Exhaust Corner } \\
\text { Turning Vanes }\end{array}$ & $\begin{array}{l}c=0.0824 m \\
t / c=0.0433\end{array}$ & $\begin{array}{l}4 \text { vanes; } \\
h / c=0.485\end{array}$ \\
\hline Vertical & Length: $0.61 \mathrm{~m}$ & $\mathrm{AR}=1.56 ;$ Acoustically \\
\hline Exhaust & Outlet: $15.24 \times 23.77 \mathrm{~cm}$ & lined perforated sheet \\
\hline Diffuser & $\theta_{\text {diff }}=8^{\circ}$ & metal interior walls \\
\hline
\end{tabular}




\subsection{Wind Tunnel Configuration Feasibility Study}

Before the design of any of the HSAWT components were fixed in complete detail, an iterative analysis was performed to assess the feasibility of implementing the desired wind tunnel configuration with the required flow conditions within the test section given the current capabilities of the existing wind tunnel. The limiting factor in obtaining certain test section speeds and mass flow rates in any blowdown wind tunnel usually lies with the usable run time of controlled steady state flow through the control valve. The higher the test section speeds and mass flow rates, the higher the capacity of compressed air required to maintain reasonable run times (mainly dictated by volume size of the reservoir tanks and storage pressure). The existing facility reservoir tanks thus pose a limit in achieving reasonable run times for a certain range of flow conditions since the storage tanks volume and pressure are fixed at 26 $\mathrm{m}^{3}$ and $100 \mathrm{psig}$ respectively. Furthermore, the cross-sectional area of the nozzle orifice will pose restrictions on achieving the maximum flow velocity target between $M=0.75$ and $M=0.8$, because larger areas will require larger mass flow rates to achieve the same test section velocity.

In order to assess if the implemented wind tunnel configuration is feasible, an unsteady analytical wind tunnel flow analysis model was developed which was used to estimate the expected mass flow rates and available run times for a given governed

flow speed within the test section. This model and analysis was developed with inspiration from Jeffries [47], who conducted a similar study when the existing wind tunnel was first commissioned at Carleton University. 


\subsubsection{Overview of the Wind Tunnel Analytical Model}

The development of a model to estimate the available wind tunnel run time and mass flow rate for a given test section flow velocity starts with the ability to simplify a typical blowdown configuration wind tunnel to a total of three isolated major components: the storage tank component; the control valve component; and the overall wind tunnel component downstream of the control valve. Figure 2.7 contains this idealized model, where the overall connection between each major component is a shared constant mass flow rate at any particular point during the run.

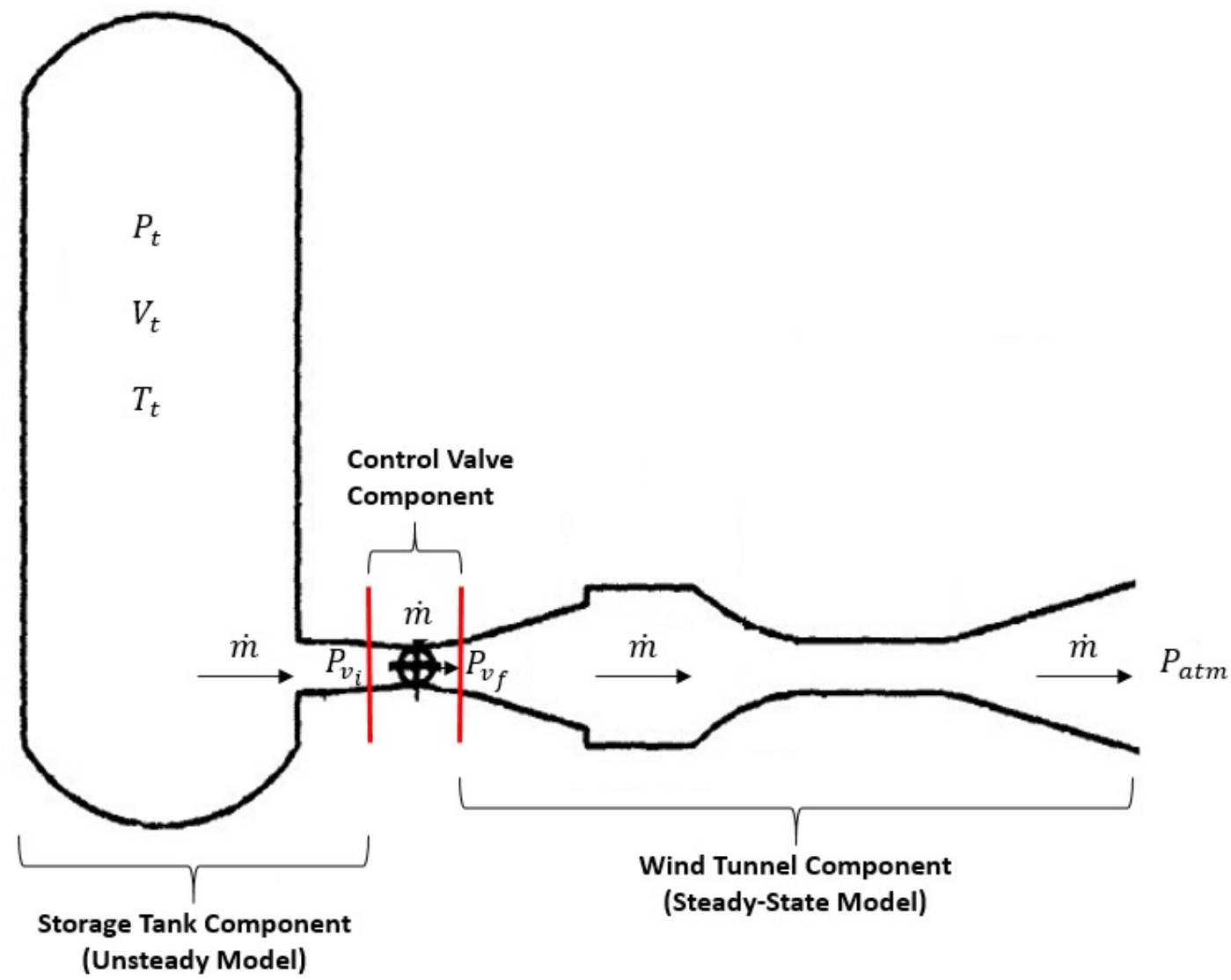

Figure 2.7: Idealized breakdown of a blowdown wind tunnel component model (adapted from [47]). 
With respect to using the idealized modeling scheme in Figure 2.7, the following assumptions apply in the analysis:

1. The additional mass flow supply of air from the compressor to the tanks while the tunnel is in operation is negligible compared to the tunnel mass flow rate, thus allowing for the storage tank component to be isolated from the supply line (confirmed by Jeffries [47]);

2. The pressure losses from the fully open safety valve and piping leading from the storage tanks to the control valve is negligible compared to the pressure losses across the control valve, thus the tank pressure, $P_{t}$, is equal to the total pressure upstream of the control valve (confirmed by Jeffries [47]);

3. The total temperature at any point in the wind tunnel is equal to the temperature of the air in the tanks, $T_{t}$ (adiabatic flow assumption);

4. The pressure and temperature in the tanks is assumed to be uniform throughout the total tank volume, $V_{t}$; where the expansion of the air out of the tanks can be analyzed as a polytropic process.

A detailed flow analysis of the wind tunnel major component is first required to determine the steady-state flow conditions at each point in the duct work, based on the required flow conditions in the test section while accounting for total pressure losses throughout the tunnel sub-components. An important output of this analysis is the estimation of the static pressure just downstream of the control valve, $P_{v_{f}}$, and the required test section mass flow rate for a governed flow speed. The modeling of the storage tank component enables the calculation of the decreasing tank pressure with respect to time as a function of the mass flow rate. This decreasing tank pressure is related to the static pressure just upstream of the control valve, $P_{v_{i}}$. Modeling 
of the control valve allows for the calculation of the required valve relative opening position which is a function of the pressure drop across the valve $\left(P_{v_{i}}-P_{v_{f}}\right)$ and mass flow rate. As the valve upstream static pressure drops due to expansion of air out of the tanks, the valve relative opening position increases to compensate and thus the wind tunnel run time can be estimated by the time it takes for the valve to fully open during a run. The relationships used to model the time varying processes of the polytropic tank expansion and the required control valve position for an estimated mass flow rate were obtained from Jeffries' outlined analysis which was conducted for the previous wind tunnel configuration [47].

\subsubsection{Overview of the Duct Component Flow Analysis}

The analysis performed to estimate the required mass flow rate and steady-state flow conditions at any point downstream of the control valve was conducted by first dividing the entire duct system into its major sub-components, where they are numerically listed as the wind tunnel datum stations. Figure 2.8 contains a schematic of the wind tunnel datum stations, where a major tunnel sub-component lies between each respective pair of stations. Table 2.3 contains a summary of the wind tunnel components between each respective labeled station in Figure 2.8.

The purpose of this is to label major sections where the expected flow conditions can be calculated based on the required flow velocity within the test section at the nozzle exit (Station 7), as well as the pressure losses through each component. Since the aim of this analysis is to estimate the maximum wind tunnel run time that can be achieved at the most limiting flow conditions, the velocity at the nozzle exit (Station 7) should represent the maximum flow velocity of interest $(M=0.8)$. The static pressure at the outlet of the wind tunnel (Station 12) is also a known and fixed 


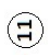

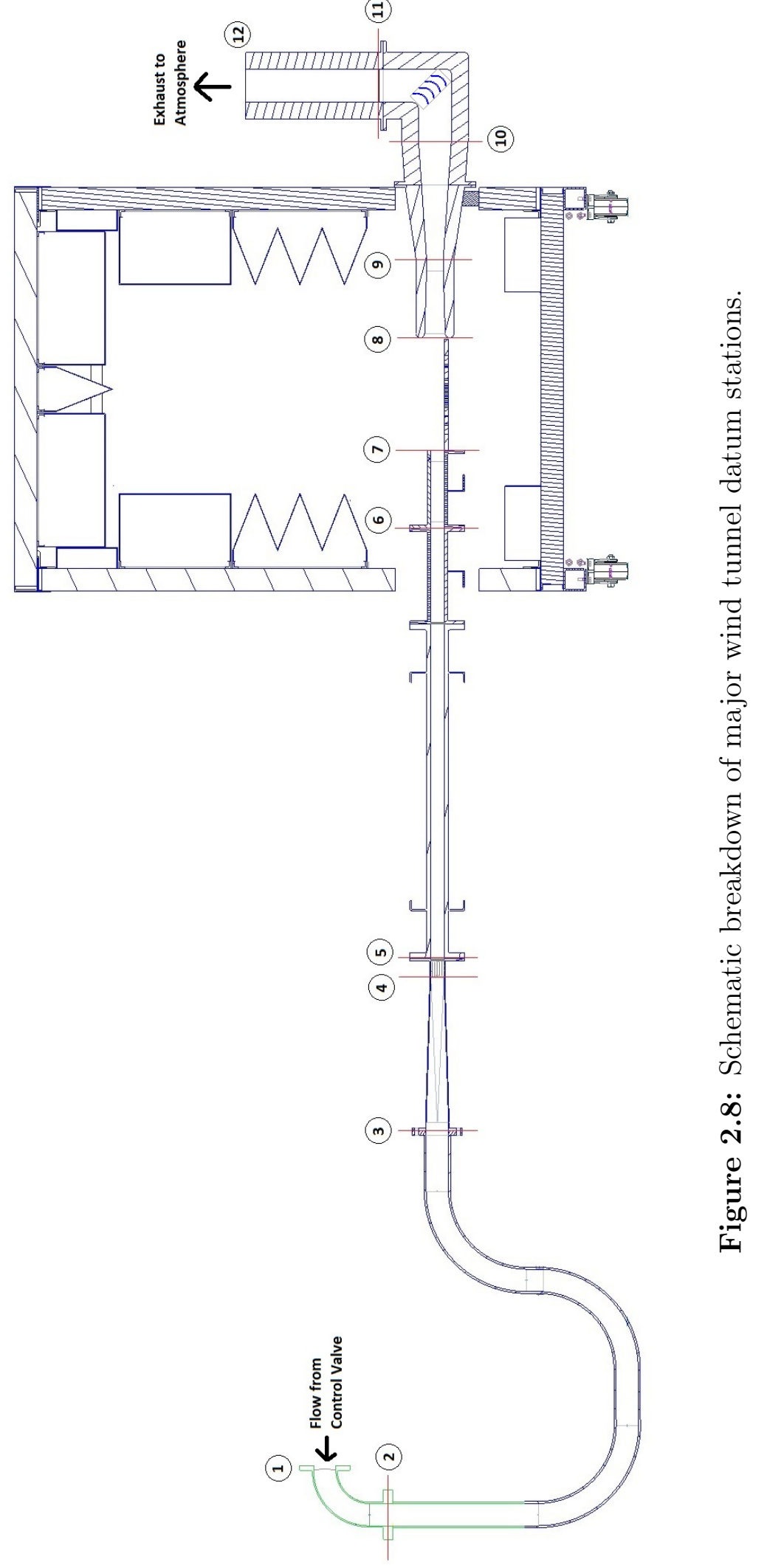


Table 2.3: Summary of wind tunnel datum station components.

\begin{tabular}{|l|c|l|}
\hline $\begin{array}{l}\text { Datum } \\
\text { Station }\end{array}$ & Component & $\begin{array}{l}\text { Known Flow } \\
\text { Conditions }\end{array}$ \\
\hline \hline $1-2$ & 90 Deg Steel Pipe Elbow & $P_{1}=P_{v_{f}}$ \\
\hline $2-3$ & Flexible High Pressure Hose & \\
\hline $3-4$ & Round-to-Rectangular Transition & \\
\hline $4-5$ & Honeycomb and 1st Screen Flow Conditioners & \\
\hline $5-6$ & Settling Duct and 2nd Screen & \\
\hline $6-7$ & Nozzle Contraction Conditioner & \\
\hline $7-8$ & Open Jet Test Section & $M_{7}=0.8$ \\
\hline $8-9$ & Jet Collector & $M_{7}=0.8$ \\
\hline $9-10$ & Collector Exhaust Diffuser & \\
\hline $10-11$ & 90 Deg Corner with Turning Vanes & \\
\hline $11-12$ & Vertical Diffuser & $P_{12}=P_{a t m}$ \\
\hline
\end{tabular}

condition at atmospheric pressure, $P_{a t m}$. The static pressure at the inlet of the 90 deg elbow, $P_{1}$, represents the static pressure just downstream of the control valve, $P_{v_{f}}$. This pressure, along with the estimated mass flow rate, is required to estimate the run time using the aforementioned analytical models of the storage tank pressure drop and required valve opening position.

Modeling of the flow conditions at each datum station first requires an estimate of the total pressure losses induced by each of the wind tunnel components, which can be represented by Equation 2.1.

$$
K=\left(\frac{P_{o_{\text {inlet }}}-P_{o_{\text {outlet }}}}{q_{\text {local }}}\right) \frac{q_{\text {local }}}{q_{7}}
$$

where the local total pressure loss coefficient, $K$, represents the normalized pressure 
loss between the inlet and outlet of the local duct component, $\left(P_{o_{\text {inlet }}}-P_{o_{\text {outlet }}}\right)$, relative to the dynamic pressure, $q$, at a location of known flow conditions (in this case at the test section corresponding to datum Station 7). The local component dynamic pressure in Equation 2.1 is usually representative of conditions at the inlet of the component, however it can sometimes be defined relative to the outlet dynamic pressure; depending on the derivation of the empirical equation used to estimate the loss coefficient.

The component pressure loss coefficients were estimated in this analysis using empirical relationships outlined by Idelchik [49], who details methods of estimating common wind tunnel duct component flow losses as a function of local duct geometry, flow velocity, and fluid properties. Since the local flow conditions at each point in the wind tunnel other than the test section are unknown, the process of estimating the flow conditions at each datum station involve the following iterative steps:

1. Calculate the test section normalized pressure loss coefficients of each tunnel component by first using an estimated guess of the local Mach number at each datum station (compressible isentropic Mach-Area relationships);

2. Calculate ratio of total pressures at each datum station with respect to the total pressure within the test section using the sum of successive in-line component pressure loss coefficients and the known flow velocity in the test section;

3. Compute new estimates of the local Mach number at each datum station by equating the compressible mass flow rate equation at the test section (with the known flow conditions and relative pressure ratios) to the compressible mass flow rate equation expressed at each component datum station;

4. Iterate steps 1 to 3 until the computed Mach number estimates at each datum station converge within a reasonable tolerance $(0.01 \%)$; 
5. Utilize the converged estimates of the local component Mach numbers, along with the known atmospheric pressure condition at the tunnel outlet, to compute the local total pressures at each datum station using the calculated total pressure ratios from step 2 ;

6. Calculate the required mass flow rate at each datum station (should be equal at every point);

7. Compute all other flow parameters of interest at each datum station using ideal gas law and isentropic flow equations.

In summary, the ultimate goal of this analysis is to obtain converged estimates of the Mach number at each point for computation of the remaining flow parameters. The ability to estimate all of the flow parameters in the wind tunnel, particularly the mass flow rate and static pressure just upstream of the control valve, serves as a requirement for estimating the available controlled run time.

\subsubsection{Wind Tunnel Feasibility Analysis Results}

Table 2.4 contains a summary of the computed flow conditions at each datum station, along with the estimated pressure losses through each wind tunnel component for a test section Mach number of 0.8. As seen in the table, the majority of the pressure losses are due to frictional effects within the flexible hose system. Other significant losses include those induced by the settling duct with the internal flow conditioning devices, as well as the growth of the shear layer within the open jet test section.

Figure 2.9 contains estimates of the required mass flow rate to govern a respective test section Mach number. The plot shows that the maximum test section Mach number of 0.8 would necessitate a tunnel mass flow rate of approximately $2.95 \mathrm{~kg} / \mathrm{s}$. 


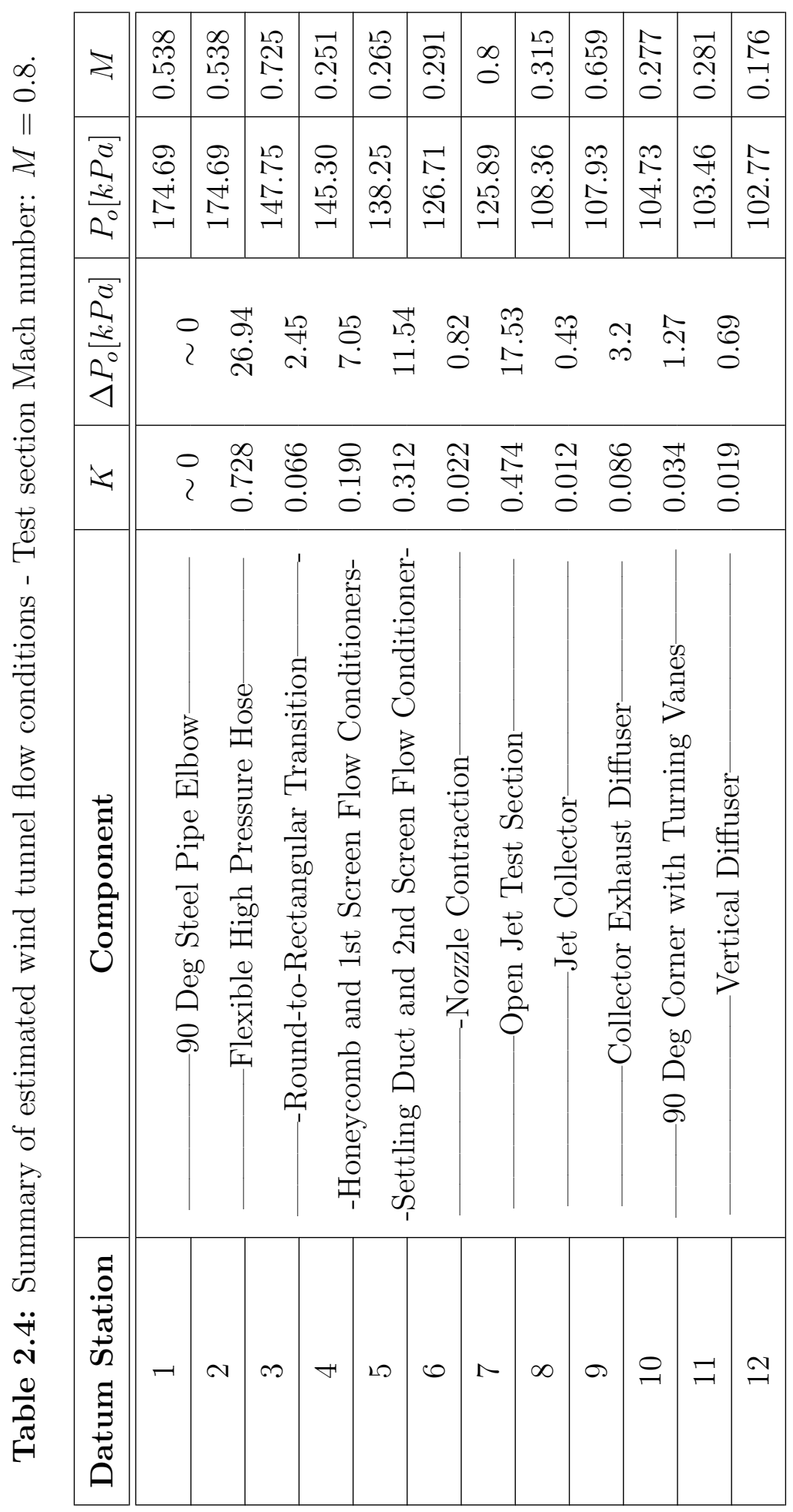


When comparing this value to the maximum governed mass flow rates utilized in wind tunnel testing with the previous configuration [47, 48, 50, this required mass flow rate is slightly approaching the maximum limits imposed by the storage tanks and control valve within the facility. Therefore, the selected nozzle outlet width and area are effectively designed at the maximum allowable with respect to the mass flow rate limits of the facility.

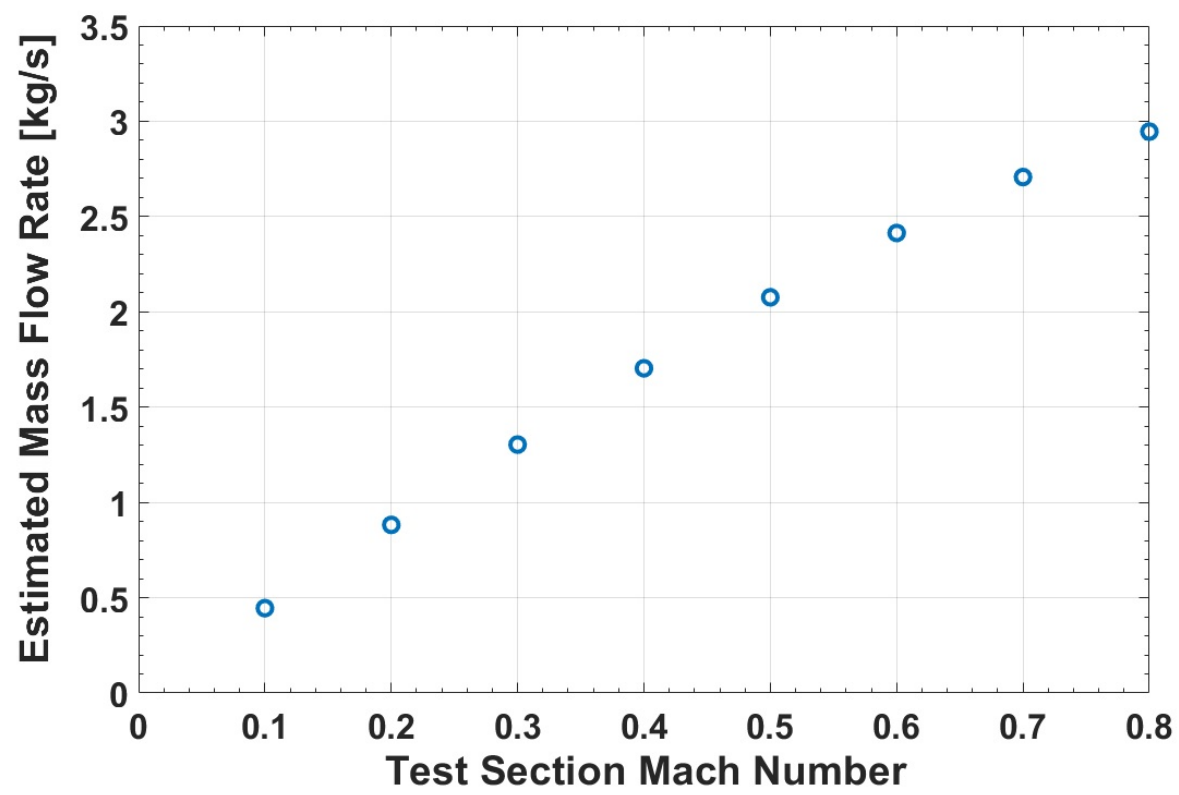

Figure 2.9: Required average wind tunnel mass flow rates for various governed test section Mach numbers.

In terms of the available controlled run time associated with these mass flow rate estimates, Figure 2.10 shows the results of the estimated run time analysis for various governed test section Mach numbers. The relationship seems to follow an exponential trend where there are no issues with run time at the lower Mach number range. However, the run time quickly decreases as higher mass flow rates are required within the test section. At the maximum Mach number of 0.8 and required mass flow rate of roughly $2.95 \mathrm{~kg} / \mathrm{s}$, a run time of approximately 50 seconds is achieved by the 
wind tunnel system. This run time is therefore sufficient when compared with the initial design requirement of at least 30 seconds of controlled flow.

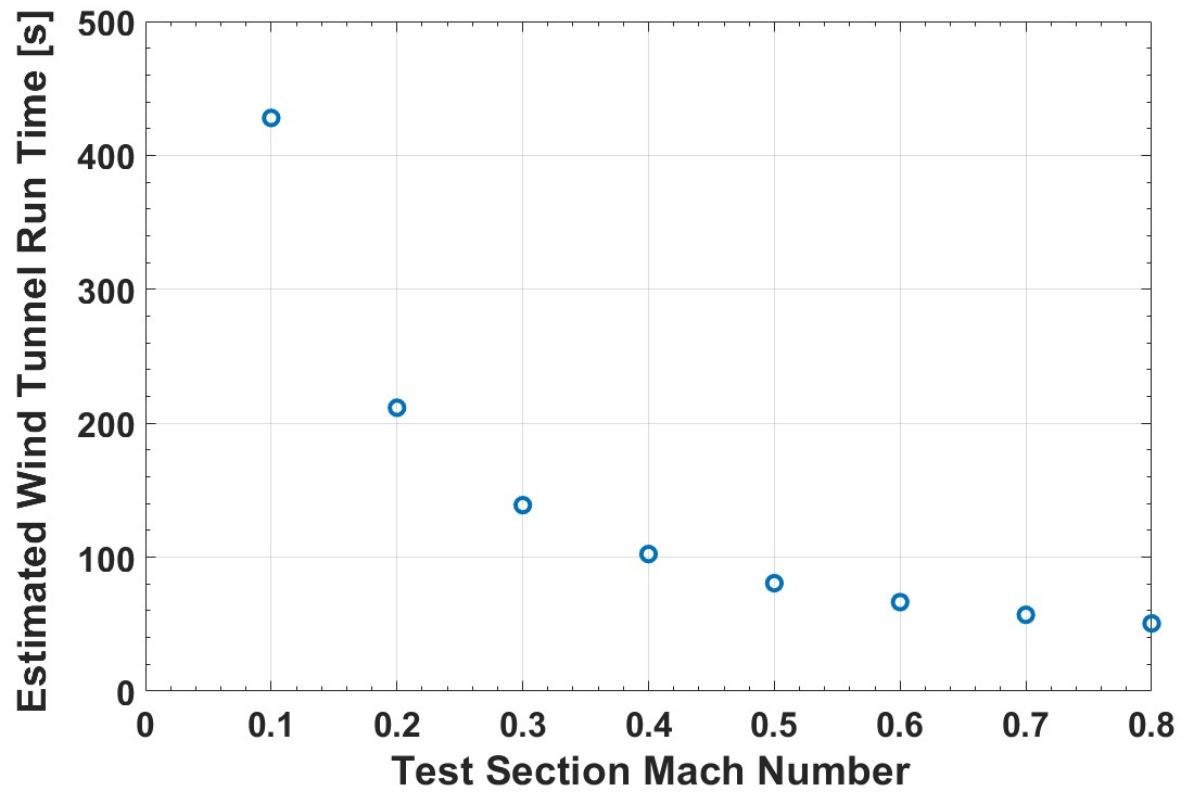

Figure 2.10: Estimated available wind tunnel run time for various governed test section Mach numbers.

\subsection{Wind Tunnel Individual Component Design}

This section provides complete details on the aerodynamic and acoustic design methodology implemented for each of the individual components in the entire wind tunnel circuit. The design of some of the components discussed below are validated with analyses conducted using numerical Computational Fluid Dynamics (CFD) simulations. The key results are provided where applicable, however please refer to Appendix A for more detailed explanations of the general computational environment, solver, and mesh domain strategy used. Details regarding parameter sensitivity and convergence studies are also outlined in Appendix A. 


\subsubsection{Flexible High Pressure Air Hose}

The purpose of the flexible high pressure hose is to deliver a flow path from the upstream wind tunnel piping (control valve) to the chamber without the need for the use of fixed piping and structural supports. This allows for some flexibility in which the position of the chamber does not need to be completely fixed. The selected hose is a RN1 flexible rubber suction/discharge hose from Hosecraft USA [51] (Figure 2.11).

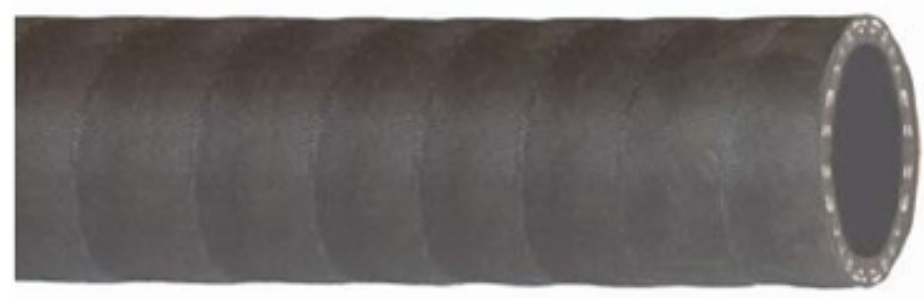

Figure 2.11: RN1 flexible suction/discharge hose (adapted from [51])

The hose is specifically designed for applications involving high pressure suction and discharge of common fluids such as compressed air. The maximum working pressure of the hose is $150 \mathrm{psi}$ which provides a factor of safety well above the expected maximum pressures seen in tunnel (roughly $30 \mathrm{psi}$ ) and even the reservoir tanks. The internal diameter of the hose matches the $10.16 \mathrm{~cm}$ diameter of the upstream wind tunnel piping and the total hose length of $7.62 \mathrm{~m}$ ensures that the hose is long enough for connection and tweaks in component positioning. The minimum bend radius of the hose is $34.29 \mathrm{~cm}$, which is sufficient for required flexibility. The hose interfaces with the piping just downstream of the control valve, as well as the transition channel through the use of flange fittings. 
From an aerodynamics and flow design perspective, the internal material, diameter, and length of the hose is crucial in terms of minimizing overall flow pressure losses. Since the diameter and length are relatively fixed design parameters for the current wind tunnel configuration, proper selection of internal hose material is important to ensure losses are minimized and that the compound can withstand continuous high speed flows without significant wear. The internal material of the selected hose consists of an Ethylene-Propylene synthetic rubber compound (EPDM), which is reinforced with textile piles and helical wire.

\subsubsection{Round-to-Rectangular Transition Duct}

The round-to-rectangular transition duct is designed to provide a smooth shift from the flexible hose to the straight channel duct. Figure 2.12 displays an internal duct schematic of the transition with the outlined chosen dimensions and shape profile.

Since the inlet and outlet areas of the transition duct are made to interface directly with other fixed components in the wind tunnel, their dimensions remained fixed in the design of this channel. The only other variables that remain are the duct length and the shape profile which provides the smooth contoured transition between the two different duct shapes and areas.

The shape of the transition channel is based off of similar designs of transition ducts in literature [44, 49]. The simplest and most effective profile shape represents the one shown in Figure 2.12, where the vertices of the rectangular cross-section are connected to adjacent points on the quadrants of the circular cross-section. This effectively produces four flat walls regions of triangular shape which are connected by the transition contoured corner pieces. 

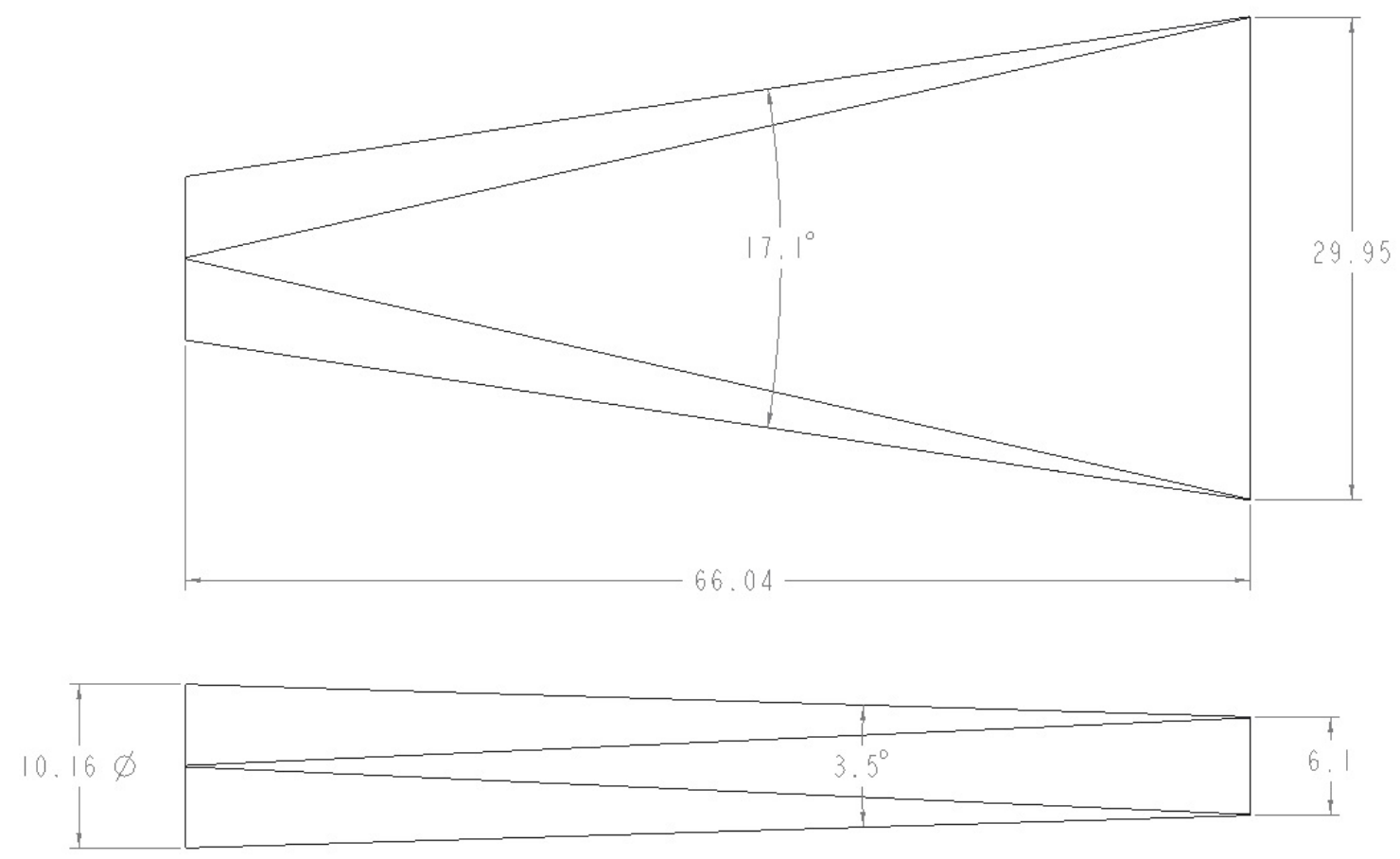

Figure 2.12: Transition channel internal duct shape and definition (all dimensions in $\mathrm{cm}$ )

Since the area ratio between the inlet and outlet of the duct is, $A R=2.26$, it operates similarly as a diffuser. It is therefore important to select a proper length, as the area change along it must be smooth to avoid any possible flow separation in the expansion plane due to the natural development of an adverse pressure gradient. Too quick of a transition will certainly cause separation along the walls, however too long of a transition will increase frictional losses, costs, and spatial limitations. Unlike normal plane diffusers, the transition duct is contracting the flow in one direction (vertical) while expanding in the other; all in a non-uniform manner. For this reason, standard diffuser design metrics or rules cannot be applied with certainty.

There is not a significant amount of transition duct design theory in literature, however, Idelchik [49] recommends a general rule of keeping the wall divergence angle in the transition duct between $20-30^{\circ}$ for a duct with a rectangular width that is 
greater than the diameter of the circular cross-section. Since this divergence angle range is quite large, and really depends on the area ratio and rectangular cross-section aspect ratio of the duct, a flow analysis using CFD was conducted to ensure that the minimum duct length (maximum wall divergence angle) was selected while avoiding significant flow separation.

A steady-state, turbulent, 3-D, Reynold-Averaged Navier-Stokes (RANS) CFD analysis was performed using ANSYS CFX. An unstructured grid consisting of tetrahedral elements using over 8.5 million nodes was used for the mesh domain of the entire 3-D transition channel. A uniform inlet velocity, representing the midpoint of the range of expected inlet speeds for the channel, of $100 \mathrm{~m} / \mathrm{s}$ was specified along with an averaged pressure outlet boundary condition. The solid walls were modeled using a no-slip condition with a refined boundary layer mesh with a first element nondimensional height of $y^{+} \leq 1$ to properly resolve for regions of possible separation. A Shear-Stress Transport (SST) turbulence model was used for the simulation.

An initial divergence angle of roughly $25^{\circ}$ was selected in the primary design, however, CFD results of the streamwise velocity contour in the divergence plane indicated regions of flow separation near the outlet. A study was conducted using multiple configurations with varying transition lengths and divergence angles. A divergence angle of roughly $17^{\circ}$, corresponding to a duct length of $66.04 \mathrm{~cm}$, was found to be the rough optimal size of the transition channel to avoid major flow separation. Figure 2.13 contains the streamwise velocity contour plot comparisons between a few of the selected divergence angles. 


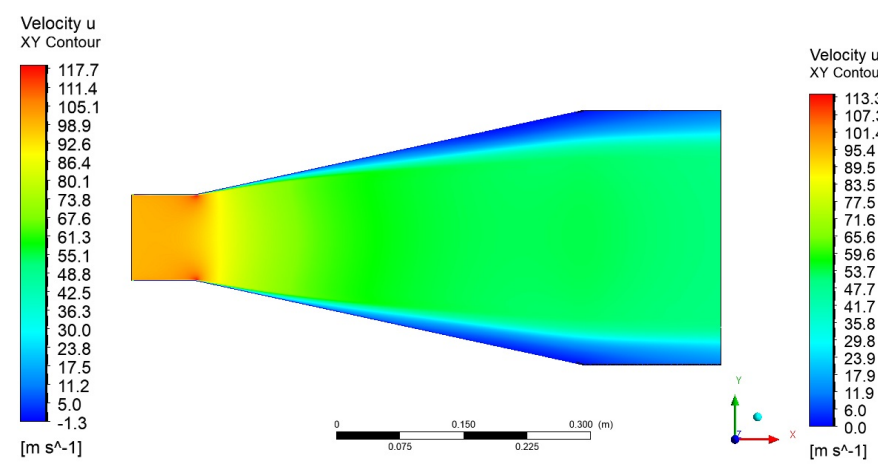

(a) $25.5^{\circ}$ Divergence Angle

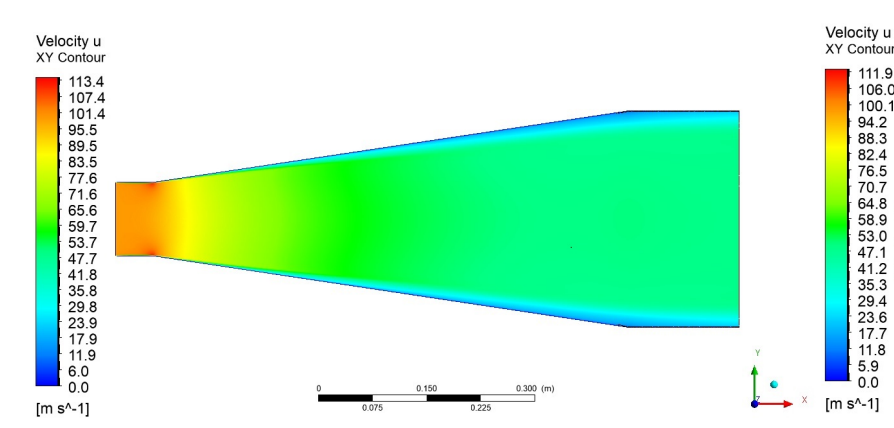

(c) $17^{\circ}$ divergence angle

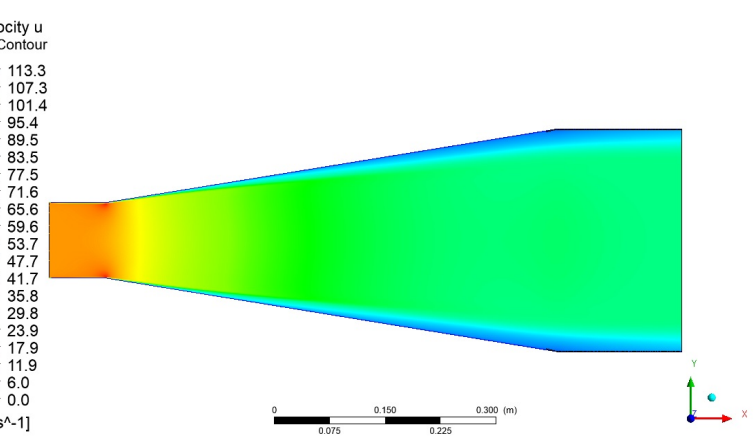

(b) $18.5^{\circ}$ divergence angle

Figure 2.13: Comparison of divergence plane streamwise velocity contour plots Transition channel CFD validation analysis

\subsubsection{Settling Duct and Flow Conditioning Devices}

A settling duct with flow conditioning devices is nominally required in any wind tunnel set-up to properly condition the flow before entering the contraction and test section. Since several components upstream of the nozzle, such as the control valve, flexible hose, and transition channel may impose large levels of flow non-uniformity and turbulence, the use of flow conditioning devices such as honeycomb cells and wire mesh screens are required to ensure the flow quality requirements are met in the test section. These conditioning devices can also improve the acoustic characteristics in the test section as they offer some sound attenuation of the propagated noise caused by upstream components such as the control valve. [43]. 
Honeycomb filters are used as a primary method to straighten the flow, as well as to attenuate some of the high frequency component of noise. Hexagonal honeycomb cells are used for their relatively low pressure drop coefficient to break up large lateral velocity fluctuations into small scale fluctuations which can then dissipate over a sufficient settling distance [52, 53]. The main requirement for the implementation of a honeycomb screen in a wind tunnel is that the average yaw angle of the incoming non-uniform flow be less than around $10-15^{\circ}$ to avoid 'stalling' of the honeycomb cells (large amount of pressure drop and reduced effectiveness) [52, 54]. It is also important to operate the honeycomb cells at sufficiently high Reynolds numbers based on cell size to ensure that the flow within the cells are turbulent (fuller turbulent velocity profile minimizes entrance/exit losses and small scale turbulence generation) [55].

In terms of physical design parameters, the ideal cell length to size ratio for honeycomb cells is between 5 and 10, with the lower end limiting the effectiveness of the honeycomb cells and the upper end inducing too large an amount of frictional losses [52 54, 56]. The cell size is selected based on the smallest lateral wavelength of the expected velocity fluctuations. Mehta and Bradshaw [52] suggest a rough amount of 150 cells per settling duct diameter to achieve proper size. With regards to the duct dimensions of the straight channel, this would correspond to a required cell size of roughly $2 \mathrm{~mm}$. Since commercial aluminum honeycomb material is readily available at certain standardized sizes and thicknesses, a honeycomb cell sheet with a compromised cell size of $3.175 \mathrm{~mm}$ and length-to-size ratio of 5 was selected for the settling duct. Figure 2.14 contains a schematic of the honeycomb cells implemented in the settling duct. 


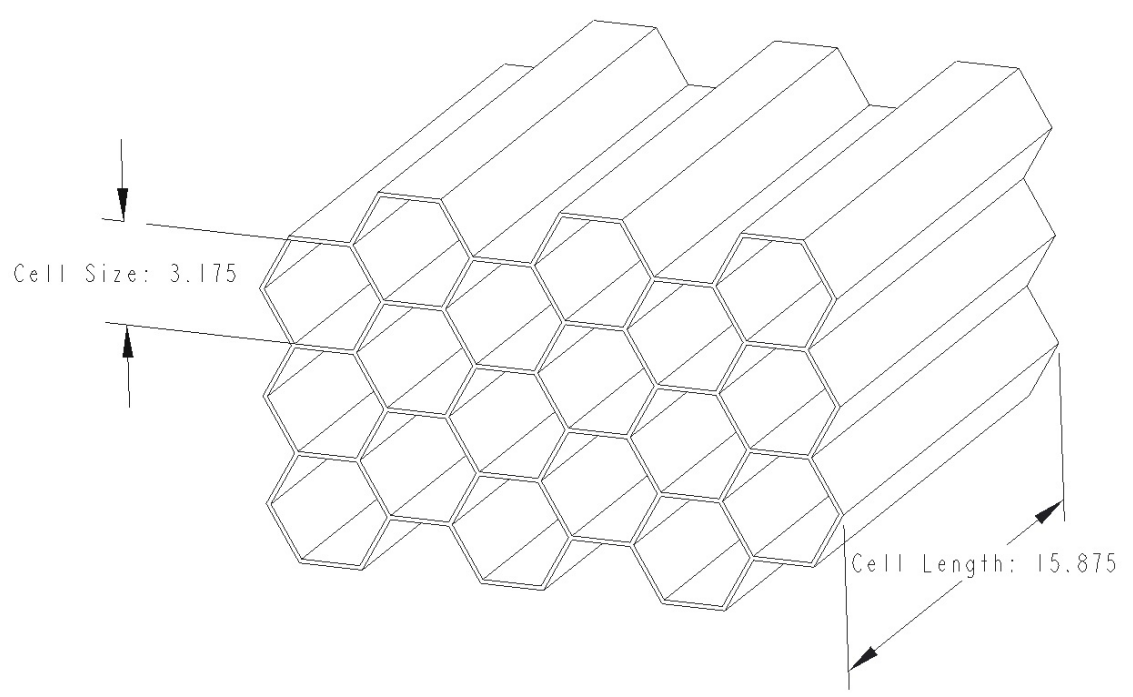

Figure 2.14: Schematic of honeycomb flow conditioning cells (all dimensions in $\mathrm{mm})$.

Along with honeycomb flow conditioning cells, stainless steel screens are commonly used for additional improvement of flow quality entering the test section. The general schematic of a flow conditioning screen is shown in Figure 2.15.

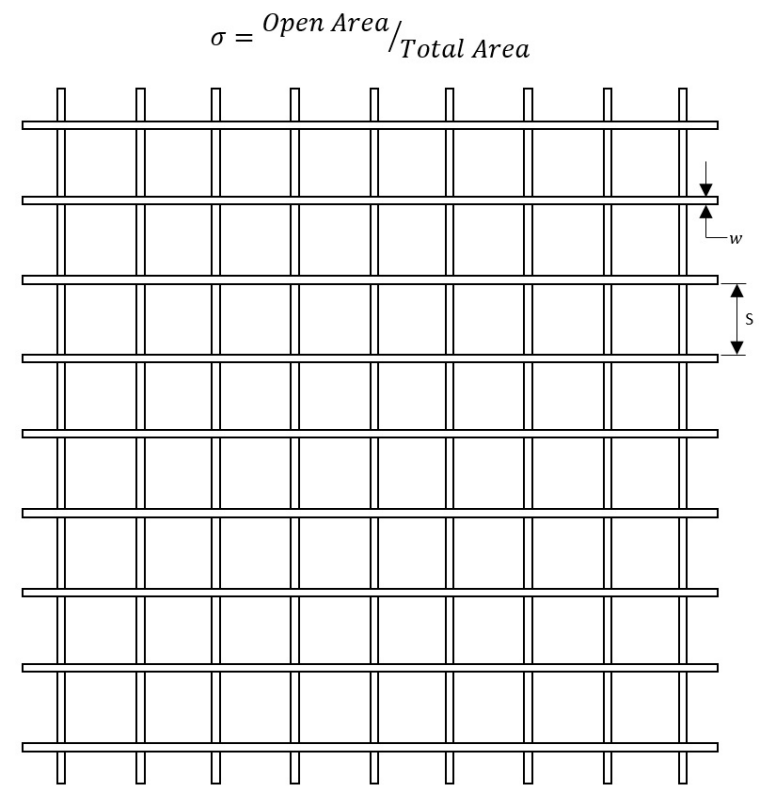

Figure 2.15: Schematic of wire mesh flow conditioning screen. 
Flow conditioning screens are usually characterized by their wire mesh diameter, $w$, their mesh cell size, $S$, and the ratio between the open flow area and total area for a given size of mesh, $\sigma$. The smaller the mesh cell size and open area ratio, the smaller the filter of incoming large scale turbulent eddies and thus reduction in overall turbulence levels. Significant pressure losses however come with the use of finer mesh screens, therefore careful selection of the number and types of screens to implement in a wind tunnel set-up must be made. Many authors suggest the use of screens with an open area ratio higher than 0.57 as a general rule to avoid large pressure losses and decreased effectiveness from the possible generation of instabilities from longitudinal vortices [57, 58]. Wieghardt [59] also suggests a settling chamber configuration with multiple screens of varying porosity (coarsest mesh to finest mesh travelling downstream within the settling chamber) can increase the efficiency in turbulence reduction and flow straightening compared to implementing only a single fine mesh screen. Adequate spacing between the final mesh and the contraction is required so that the small scale fluctuations from the screen are dissipated. Mehta and Bradshaw [52] suggest that this distance should be no less than $20 \%$ of the duct channel width. An additional concern for the use of flow screens in high speed flows is the Mach number of the flow inside the settling chamber. It is crucial to ensure that a sufficiently large screen open area ratio is chosen so that it is not operating at or close to choked conditions where large increases in pressure losses occur [60].

Table 2.5 contains a summary of the selected design parameters for the two screens in the settling duct. Both screens are stainless steel commercial screens which are cut to proper size for the settling duct (straight channel assembly) and held in place by the bolted flange connections at the straight channel inlet and outlet faces. 
Table 2.5: HSAWT flow conditioning screen design parameters.

\begin{tabular}{cccc}
\hline Screen & $\mathbf{w}[\mathbf{m m}]$ & $\mathbf{S}[\mathbf{m m}]$ & $\sigma$ \\
\hline \hline 1 - Upstream Screen & 0.1651 & 0.68072 & 0.65 \\
2 - Downstream Screen & 0.1397 & 0.46482 & 0.59 \\
\hline
\end{tabular}

\subsubsection{Chamber Inlet Nozzle Section}

Wind tunnel nozzles or contractions are designed to accelerate the flow from the settling duct into the test section at the required velocities of interest. The contraction ratio $(\mathrm{CR})$, overall length, and wall contour shape all affect the degree of flow uniformity, turbulence intensity reduction, and boundary layer growth of the flow entering the test section [43, 52, 61]. Ideally, nozzles should be designed to be as short as possible to minimize frictional pressure losses while also containing a large contraction ratio which aids in attenuating both mean and fluctuating velocity variations. Careful design of the shape of the wall contours must also be considered as separation can occur in regions of sharp changes in streamline curvature, thus leading to poor test section flow quality [61 63]. Possible separation in contractions can be visualized by examining the typical normalized wall static pressure profile along the contour of a nozzle shown in Figure 2.16 [44].

The regions outlined by the blue circles in the wall static pressure distribution describe regions with positive slopes of wall static pressure; which indicates regions of adverse pressure gradients and possible flow separation. The orange dotted lines show where these regions correspond to the physical locations in a typical contraction profile. Although separation hardly occurs in the first region closest to the nozzle inlet, the second region can contain a large positive pressure gradient if the inflection point of the contraction profile is too abrupt. Morel [61] suggests that the maximum 

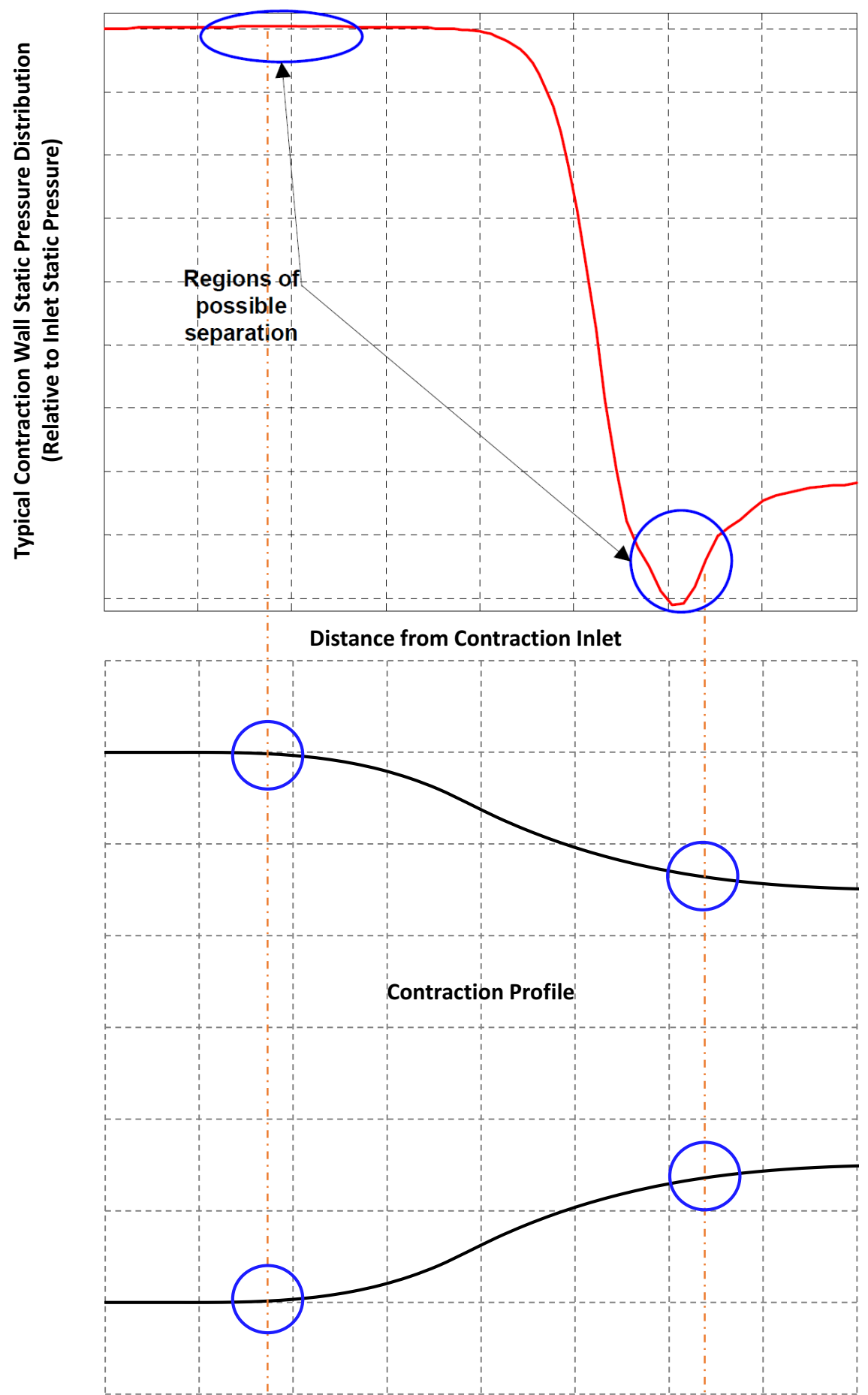

Figure 2.16: Visualization of possible regions of separation in a general contraction profile (adapted from [44]). 
physical contour slope at any point along the nozzle walls should not exceed $35^{\circ}$.

With regards to the design of a suitable contraction, the inlet width of the nozzle, $W_{n_{i}}$, is a fixed parameter which corresponds to the $30 \mathrm{~cm}$ width of the straight channel assembly (settling duct). For simplicity, the height of the channel was selected to remain constant with the straight channel at $6.1 \mathrm{~cm}$. This effectively implies a 2-D contraction design where the height of the channel is fixed and the walls of the nozzle reduce from the $30 \mathrm{~cm}$ entrance width to some smaller value based on the selected contraction ratio. The nozzle outlet width, $W_{n_{e}}$, and contraction ratio are therefore a variate design parameters in this analysis.

The selection of the nozzle exit width and overall contraction ratio was done based on numerous factors. The first criterion involved selecting the largest possible contraction ratio which leads to benefits in terms of turbulence intensity and flow non-uniformity reduction in the test section. Another factor includes the overall required usable test section space, in which increasing the contraction ratio will make less use of the microphone array on the test panel for possible spanwise correlation measurements. A final consideration is the size of the nozzle outlet area, as larger areas will require larger mass flow rates, and thus smaller wind tunnel run times, to obtain the same test section speeds. With all of this in mind, a contraction ratio of 2 was selected which results in an outlet width of $15 \mathrm{~cm}$. This $\mathrm{CR}$ value essentially corresponds to the lower limit of basic 2-D nozzle designs in literature [61, 62]. The nozzle outlet width is also at the smallest dimensions in which the bulk of the flush mounted sensor port locations can be used. The wind tunnel flow analysis used to estimate the mass flow rate and approximate wind tunnel run times for the selected nozzle outlet size also indicated that it was approaching the maximum possible for appropriate flow conditions and controlled run times at the maximum required test section speeds (refer to Chapter 2.3). 
With the inlet and outlet widths of the nozzle fixed, the selection of the proper length and wall contour profile followed a suggested design method outlined by Morel [61] for 2-D contractions. He proposes that the optimum profile for 2-D contractions involves a matched cubic profile, in which the inlet and outlet portion of the nozzle contour consists of two separate 3rd order polynomials which are matched at some inflection point at some distance down from the nozzle inlet. Figure 2.17 displays a schematic describing this contraction profile with the definitions of relevant dimensional parameters. The total length of the nozzle is $L_{n}$ and the match point of the cubic profiles occurs at a fractional distance of the nozzle length from the inlet, $X_{m} L_{n}$.

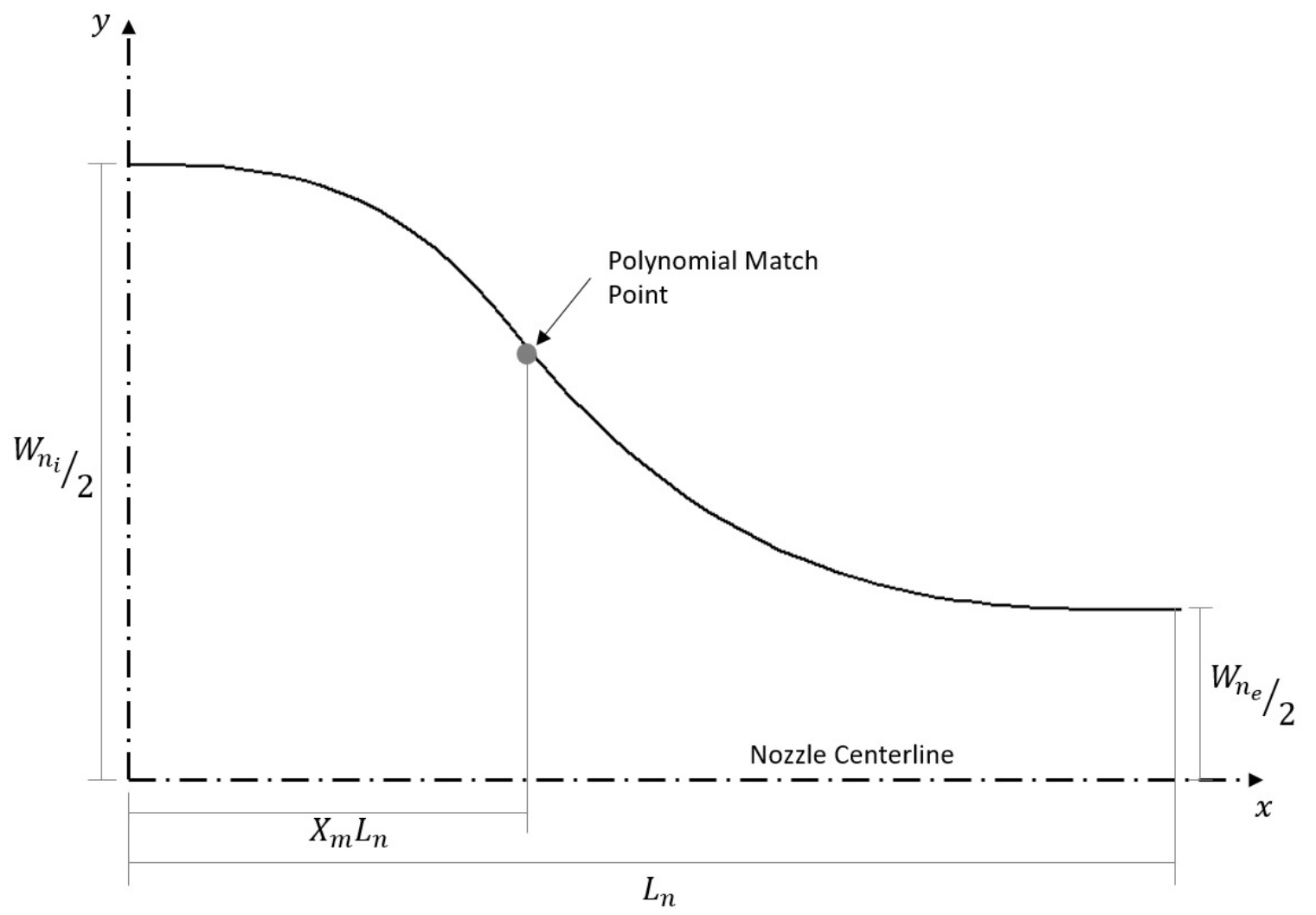

Figure 2.17: Schematic of cubic matched nozzle profile. 
Morel [61] outlines a complete numerical approach to the design of 2-D contractions using developed charts and equations from systematically compiled computational data from various nozzles of varying contraction ratios, lengths, and $L / W_{n_{i}}$ ratios. For a given design contraction ratio of 2-4, the optimum fractional match point distance from the inlet, $X_{m}$, can be determined from the following implicit relationship shown in Equation 2.2.

$$
X_{m}^{1 / 2}\left(1-X_{m}\right)^{-2 / 3}=F_{e}^{1 / 3} G_{i}^{-1 / 2} C R^{1 / 2}(C R-1)^{1 / 6}
$$

where $F_{e}$ and $G_{i}$ are design parameters which are functions of the nozzle contraction ratio and expected levels of normalized wall static pressure which will avoid separation based on Stratford's criterion [64]. Typical values for these parameters for a contraction ratio of 2 are outlined in the nozzle design charts provided by Morel [61] and correspond to 0.4 and 1.65 respectively.

Once Equation 2.2 is solved implicitly for $X_{m}$, the optimum nozzle length $L_{n}$ is determined from Equation 2.3 [61].

$$
\left(\frac{L_{n}}{W_{n_{i}}}\right)^{3}=\left(\frac{C R-1}{C R}\right) \frac{1}{G_{i} X_{m}}
$$

With the length and match point of the nozzle profile defined, Equations 2.4a and $2.4 \mathrm{~b}$ are used to compute the nozzle profile coordinates, $\left(x, W_{n}\right)$, before and after the match point defined by $X_{m}[61$.

$$
\frac{W_{n}-W_{n_{e}}}{W_{n_{i}}-W_{n_{e}}}= \begin{cases}1-\frac{1}{X_{m}^{2}} \frac{x^{3}}{L_{n}^{3}} & \text { for } x / L_{n} \leq X_{m} \\ \frac{1}{\left(1-X_{m}\right)^{2}}\left(1-\frac{x}{L_{n}}\right)^{3} & \text { for } x / L_{n}>X_{m}\end{cases}
$$


Figure 2.18 contains the fixed design for the internal nozzle duct shape and profile with relevant physical dimensions outlined. The computed optimal parameters of nozzle length and match point are $L_{n}=27.46 \mathrm{~cm}$ and $X_{m}=0.3617$ respectively.

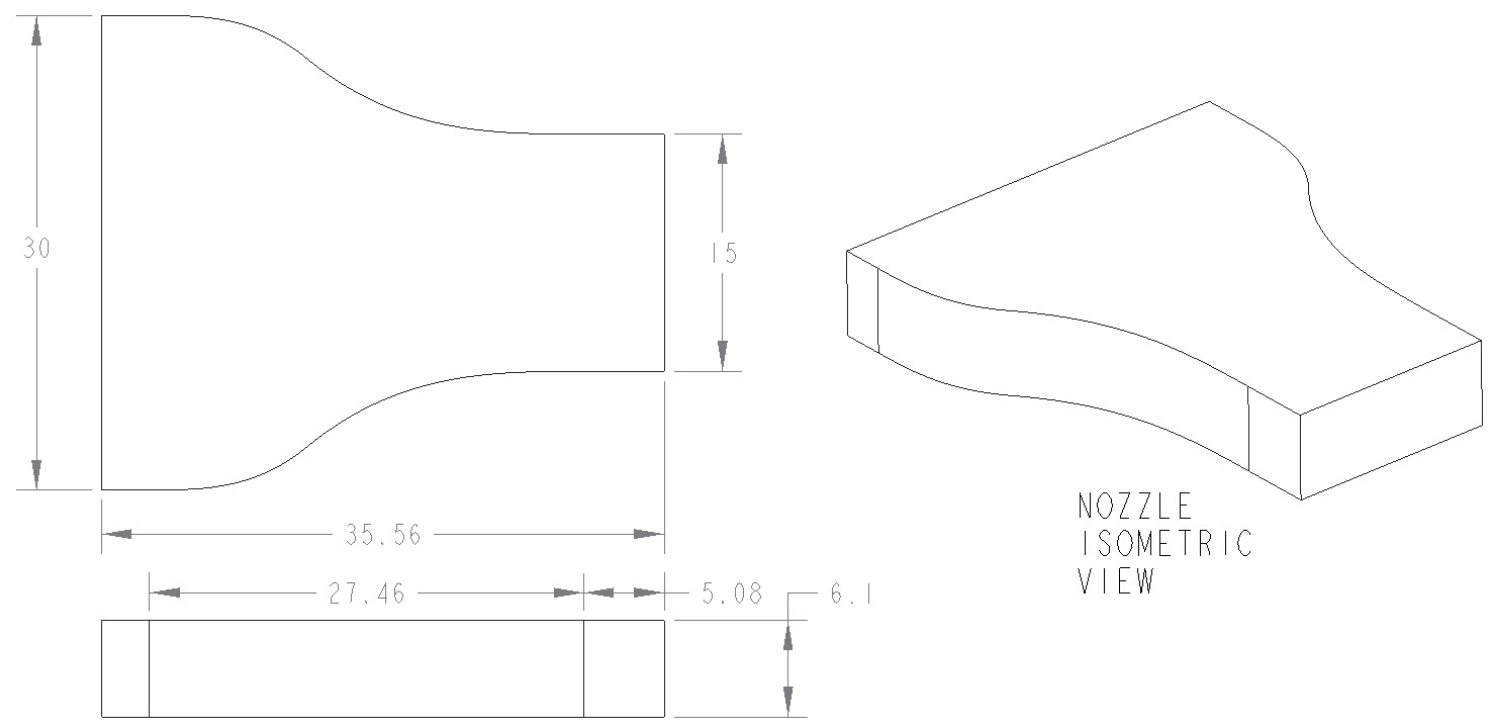

Figure 2.18: Nozzle channel internal duct shape and definition (all dimensions in $\mathrm{cm})$.

A CFD analysis was performed to validate the final design of the contraction profile in terms of flow separation and outlet flow quality parameters. A similar 3-D, steady-state, turbulent CFD analysis was done with analogous boundary conditions as implemented in the transition channel analysis. The mesh consisted of roughly 3.6 million unstructured hexahedral elements. For further information regarding the computational domain and parameters selected, please refer to Appendix A,

Although several uniform inlet velocity conditions were analyzed, Figure 2.19 contains the streamwise velocity contour plot in the contraction plane for an outlet speed roughly in the middle of the expected operating range of the nozzle channel.

The contour plot shows no regions of separation in the aforementioned possible affected areas. The results from the plots of the streamwise wall static pressure and 


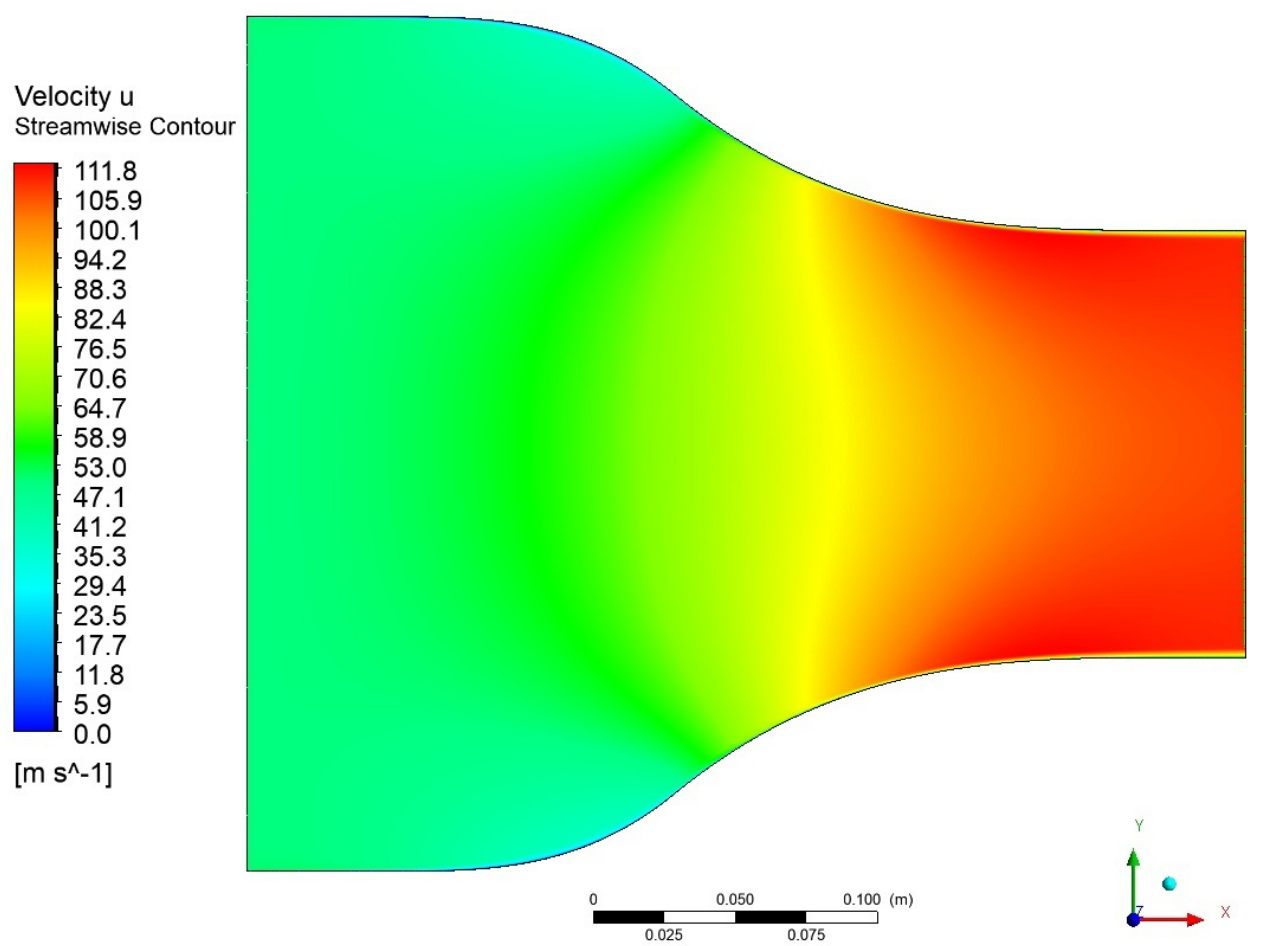

Figure 2.19: Contraction plane streamwise velocity contour plot - Nozzle channel CFD validation analysis.

wall shear stress profiles shown in Figure 2.20 further ensure that there are no regions of separation along the wall due to a relatively small magnitude of positive wall static pressure gradient and positive values of wall shear stress.

Figure 2.21 contains CFD results regarding key performance parameters at the spanwise outlet plane of the nozzle to help assess flow quality entering the test section. The plot of the flow uniformity index (ratio of local streamwise outlet velocity to the average streamwise velocity across the entire outlet) displays a range remaining within $\pm 1 \%$ of the average freestream velocity. The plot of the outlet turbulence intensity indicates that the nozzle is capable of reducing streamwise turbulent fluctuations up to $90 \%$ for an inlet TI of $1 \%$. 


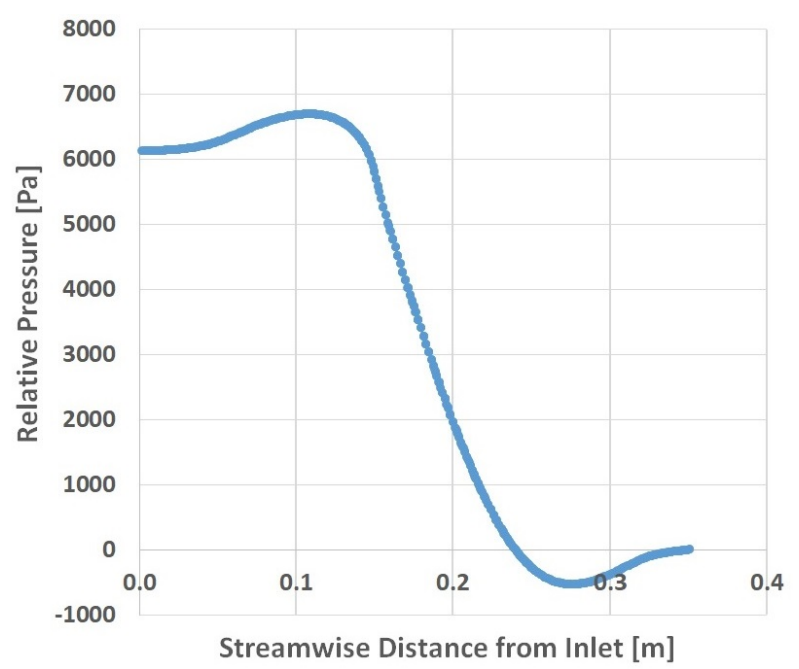

(a) Wall static pressure profile

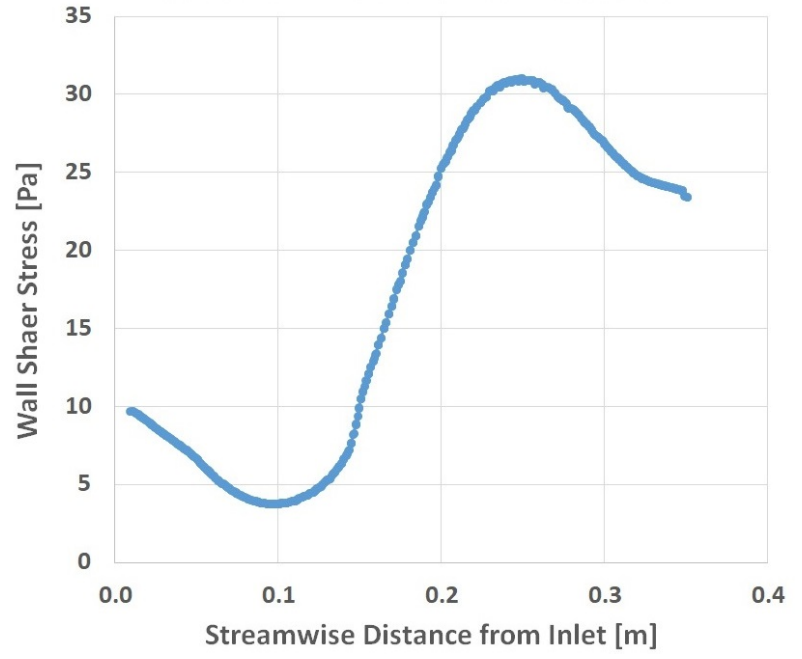

(b) Wall shear stress profile

Figure 2.20: Streamwise nozzle wall parameter data - Nozzle Channel CFD validation analysis.

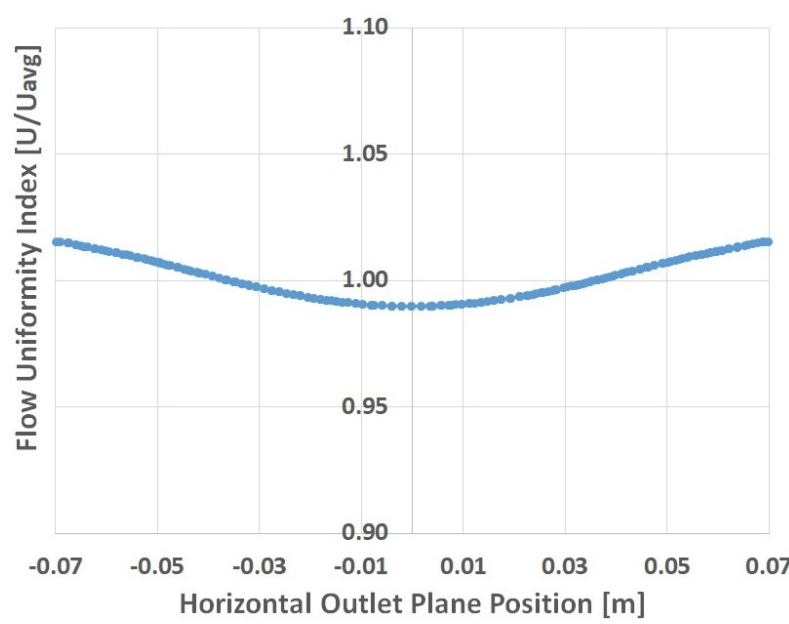

(a) Outlet flow non-uniformity profile

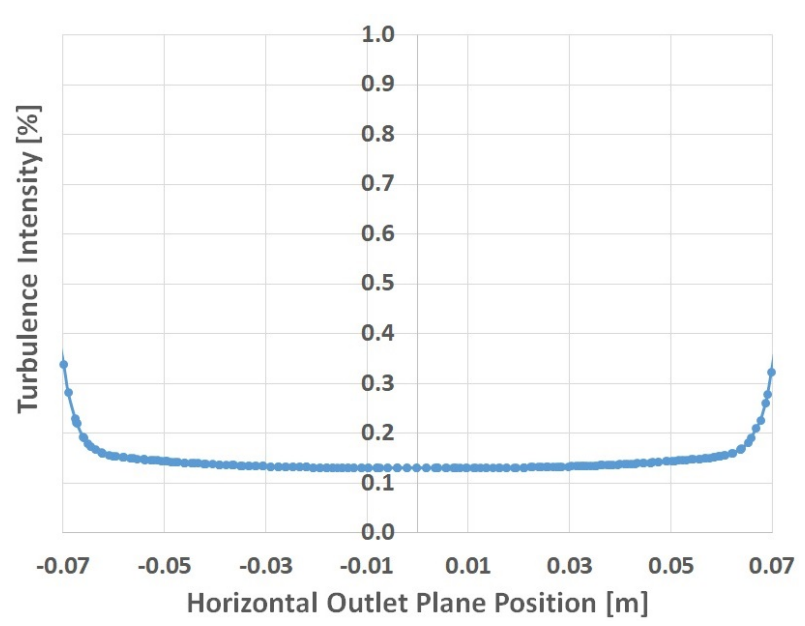

(b) Outlet turbulence intensity profile

Figure 2.21: Outlet centreline plane spanwise parameter data - Nozzle Channel CFD validation analysis. 


\subsubsection{Anechoic Test Chamber}

The test section anechoic enclosure was designed in conjunction with consultation from Eckel Industries Inc. Eckel is a leading manufacturer of noise control solutions and acoustic enclosures. Figures 2.22 and 2.23 contain detailed drawings of the chamber with relevant dimensions outlined.

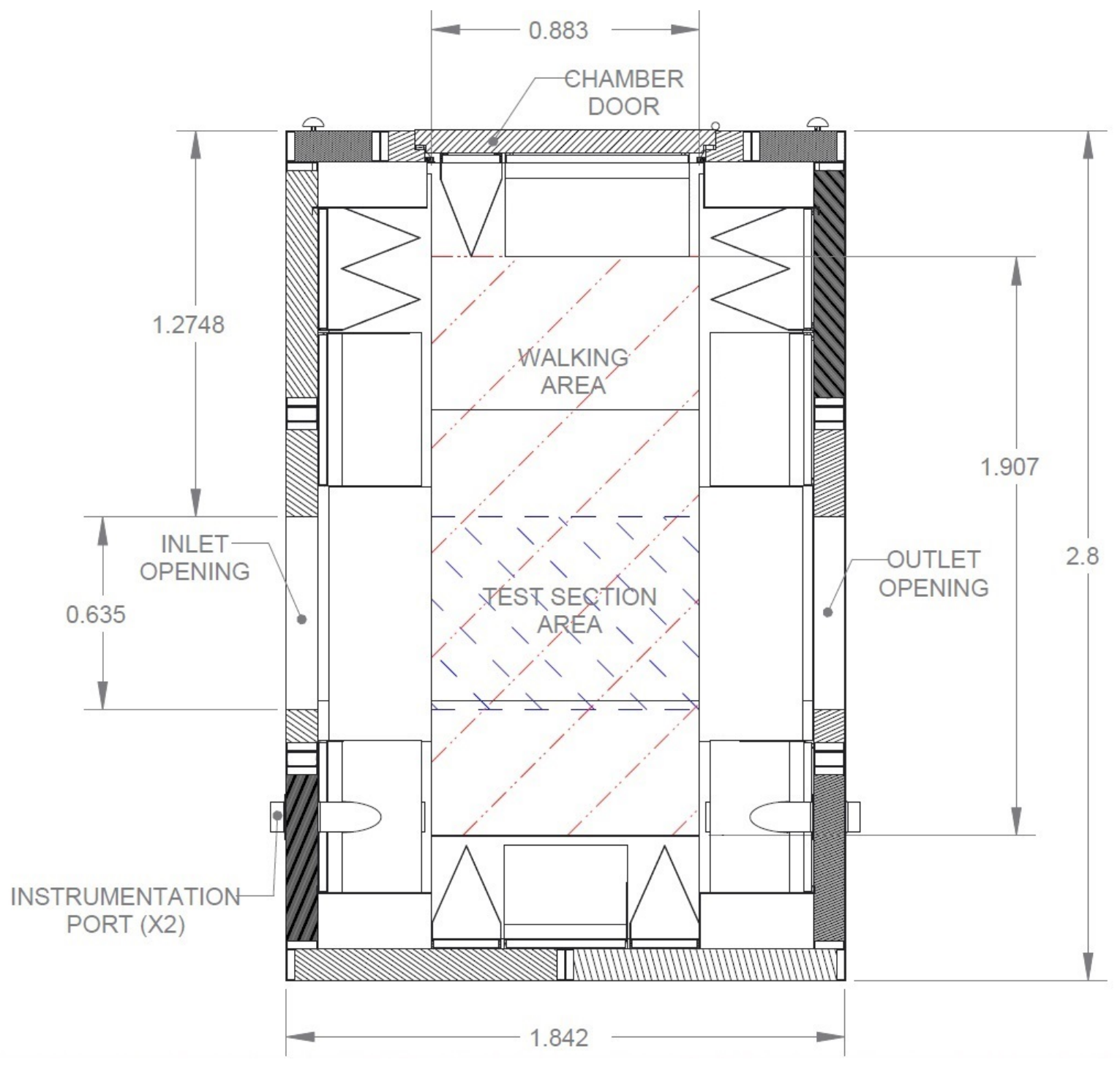

Figure 2.22: Test chamber plan view schematic (all dimensions in $\mathrm{m}$ ). 


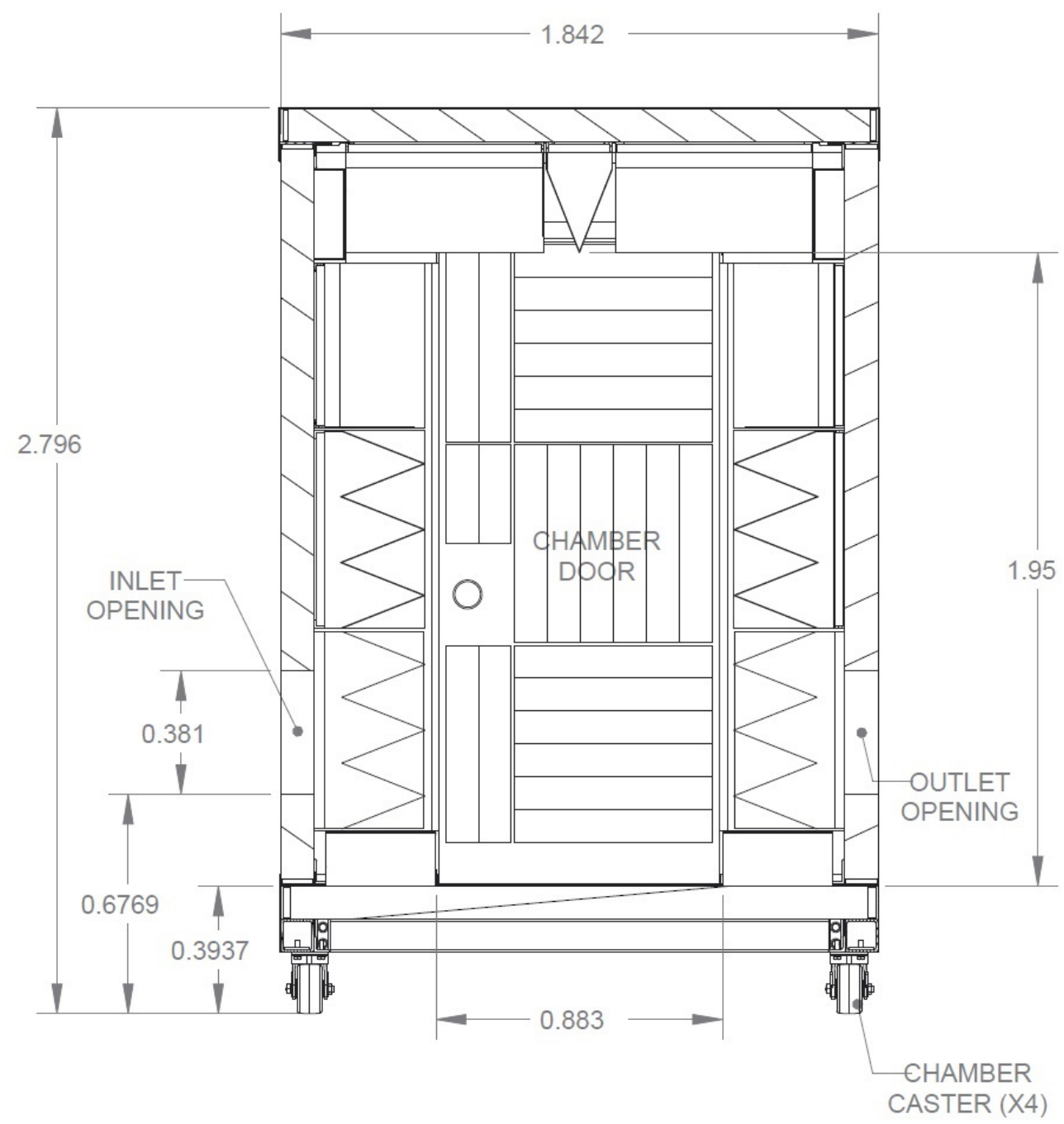

Figure 2.23: Test chamber elevation view schematic (all dimensions in $\mathrm{m}$ ).

The chamber was first designed in mind with the spatial limitations in the facility. This included the requirement for adequate space to store the chamber, space to move it around the facility safely, and room to manoeuvre around the outside of the chamber when in use. These requirements essentially fixed the maximum possible outer dimensions of the chamber to $2.8 \mathrm{~m}$ in width and $1.842 \mathrm{~m}$ in depth. The chamber walls are designed with standardized acoustic panels which are $10.16 \mathrm{~cm}$ thick and contain fiberglass insulation to help attenuate background noise levels inside the chamber. The acoustic wedges comprise of fiberglass bulk insulation wrapped 
around in fiberglass cloth and enclosed in perforated sheet metal which forms the wedge shape. The wedges are of standard size for anechoic chambers with a cut-off frequency of $250 \mathrm{~Hz}$, which corresponds to a total wedge depth from the wall of 34.3 $\mathrm{cm}$. The selection of the outer planform dimensions of the chamber, the acoustic wall panels, and the wedges thus fix the internal planform dimensions of the chamber to $1.907 \mathrm{~m}$ in width and $0.883 \mathrm{~m}$ in depth of usable space. With the floor wedges removed from the chamber, the total height inside from the floor to the ceiling wedge tips is $1.95 \mathrm{~m}$, which provides enough room for a person to walk around inside the chamber comfortably. An acoustic door fitted with gasket seals allows access to one side of the chamber. The wall cut-outs on each side of the inlet and outlet walls of the chamber allows for the nozzle and exhaust assembly components to be fed into the chamber. The holes are of rectangular shape with a width and height of $63.5 \mathrm{~cm}$ and $38.1 \mathrm{~cm}$ respectively. The cut-outs are located at a position that is offset from the centre of the chamber inlet and outlet walls (refer to Figure 2.22). The main purpose of this is to enable future test research capabilities using flow visualization techniques where adequate room is required on one side of the test section for this equipment to be placed. The side of the test section with the door was selected as the area with the larger end for ease of access, as well as to provide enough space for the chamber door to be opened without striking any of the facility walls (refer to Figure 2.5.

The entire chamber is designed to attenuate noise above the wedge specified cutoff frequency where the acoustic energy absorption is $99 \%$ or higher and the ratio of reflected sound pressure to incident sound pressure is below 10\% [34]. The ability of the chamber to reduce background noise levels is dictated by its transmission loss, $T L$, which is given on the decibel scale by:

$$
T L[d B]=L_{p_{i}}[d B]-L_{p_{f}}[d B]=20 \log _{10}\left(\frac{p_{r m s_{i}}}{p_{r m s_{f}}}\right)
$$


where $L_{p_{i}}$ and $L_{p_{f}}$ are the sound pressure levels (dB relative to $20 \mu \mathrm{Pa}$ ) inside and outside of the chamber respectively; and $p_{r m s_{i}}$ and $p_{r m s_{f}}$ are the root-mean-square fluctuating sound pressures inside and outside of the chamber.

The expected noise reduction levels or transmission loss for the chamber are listed in Table 2.6 for certain octave band centre frequencies of interest. These values are sufficient enough to provide adequate background noise levels inside the chamber that are lower than the expected response from the TBL based on semi-empirical model predictions and facility noise environment microphone measurements.

Table 2.6: Anechoic chamber noise reduction profile (based on data from [34])

\begin{tabular}{c|cccccccc}
\hline \hline Frequency $[\mathrm{Hz}]$ & 62.5 & 125 & 250 & 500 & 1000 & 2000 & 4000 & 8000 \\
\hline Transmission Loss $(T L)[d B]$ & 25 & 38 & 58 & 59 & 60 & 62 & 64 & 55 \\
\hline \hline
\end{tabular}

Other chamber design aspects of interest include two instrumentation feed-through ports, lighting fixtures, and wire mounting hooks. The chamber is also fitted with two ventilation holes $(15.24 \mathrm{~cm}$ diameter $)$ on the ceiling of the chamber to allow for some pressure equalization when in operation at high test section flow speeds. Some alternative acoustic test configurations and research also require these ventilation holes to allow entrainment of air inside the chamber. These holes are fitted with the ventilation silencers to help suppress internal noise contamination.

\subsubsection{Chamber Ventilation Silencers}

Acoustic silencers are devices used to provide a transmission loss of acoustic sound pressure levels from one side of a duct to the other [65, 66]. These silencers are connected in-line and contain interior walls that are lined with sound-absorbing media to help dissipate sound travelling through the duct work. Silencers can be constructed in 
many ways, however for low speed ventilation applications, plenum type silencers are most appropriate [66]. Plenum silencers contain an acoustically treated chamber with an inlet and outlet on opposite sides corresponding to the largest possible distance between both openings. The larger the chamber and the thicker the acoustic insulated lining, the larger the transmission loss through the silencer. For efficiency in terms of reducing the overall size of the plenum while achieving similar performance, internal acoustic absorptive baffles can be placed in such a way to impede direct line-of-sight transmission of the acoustic waves through the silencer. Figure 2.24 contains three general types of plenum silencer designs to illustrate this concept [67.

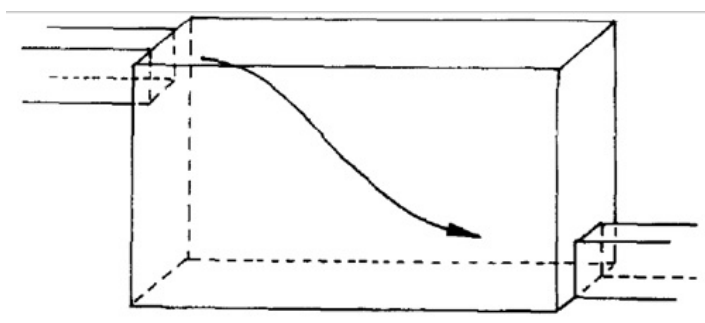

(a) Single-Pass plenum

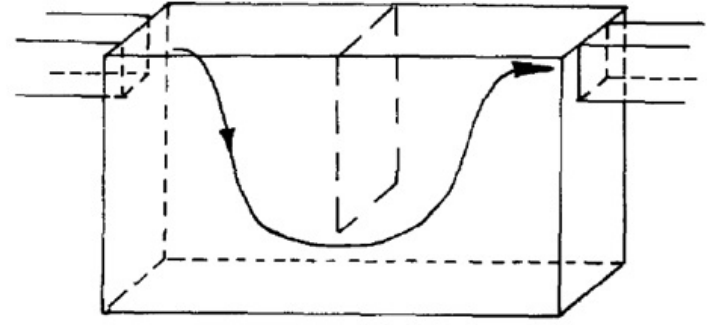

(b) Two-pass plenum (single absorptive baffle)

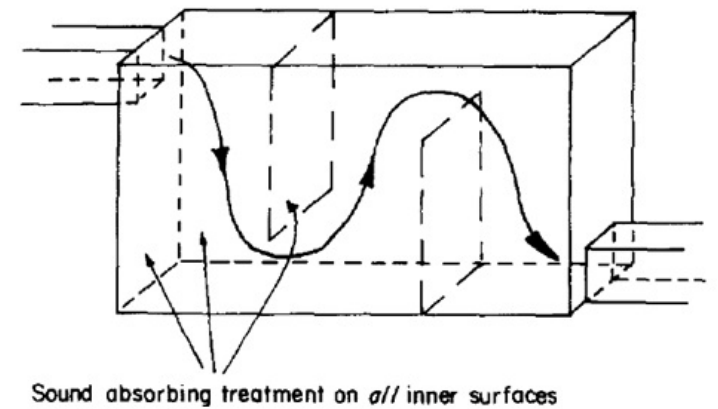

(c) Three-pass plenum (two absorptive baffles)

Figure 2.24: General plenum silencer design configurations (adapted from [67]).

All internal surfaces of the silencer are constructed generally of acoustic bulk fiberglass insulation, covered by fiberglass cloth and encased in acoustically transparent perforated sheet metal. This is similar in construction to the acoustic wedges inside 
the anechoic chamber. Proper selection of the perforated sheet metal is important as too low of an open area ratio, $O A$, and too large of a hole spacing, $s$, can lead to an internal duct lining that is not acoustically absorptive; thus resulting in poor silencer performance [68]. Schultz [68] provides a method to calculate how acoustically transparent a type of perforated sheet metal is based on its transparency index, $T i$, which is determined as follows:

$$
T i=\left(\frac{0.04(O A)}{\pi t_{p e r f} s^{2}}\right)
$$

where $O A$ is the open area ratio expressed as a percentage; $t_{\text {perf }}$ is the thickness of the sheet metal in inches; and the hole spacing, $s$, is expressed in inches. Schultz recommends a value of $T i$ no less than 2000 so that the surface is acoustically transparent for proper sound absorption from the enclosed fiberglass material [68].

The final design of the HSAWT chamber silencers consisted of a two-pass plenum design with a 4" thick acoustic duct lining and internal baffle. The internal planform size of the plenum was selected based on the maximum allowable space to fit on top of the chamber. The internal duct height of the silencer is constant at $15.88 \mathrm{~cm}$. Figure 2.25 contains a drawing schematic with relevant dimensions and component labels.

A method to predict the performance of a two-pass silencer of a certain internal plenum size outlined by Cummings [67] was used to validate the design. Figure 2.26 shows a plot of the predicted transmission loss of each silencer as a function of frequency. The plot shows a sufficient and constant transmission loss of around 40 $d B$ at all frequencies above $250 \mathrm{~Hz}$. 


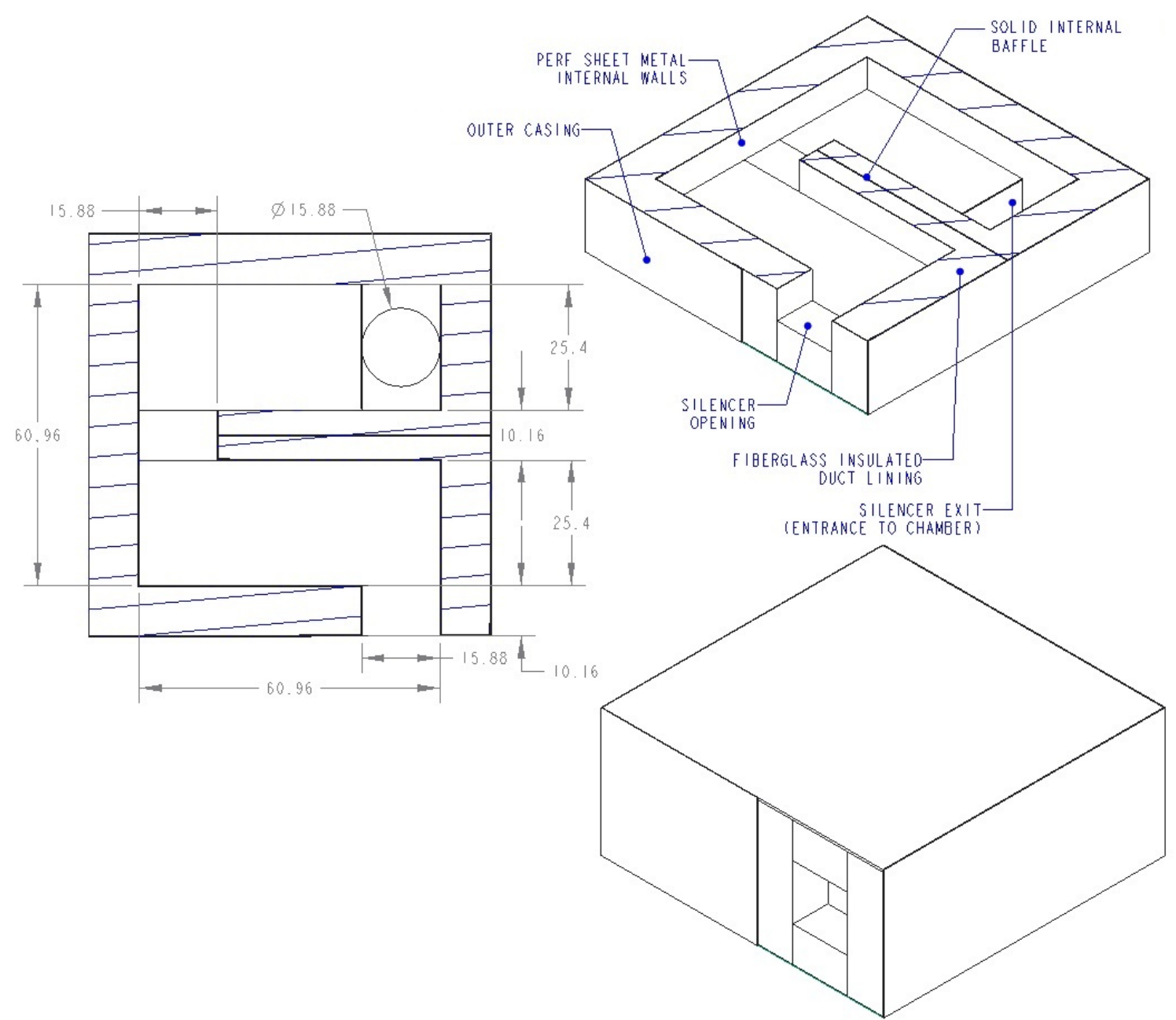

Figure 2.25: Chamber ventilation silencer design schematic (all dimensions in $\mathrm{cm}$ ). 


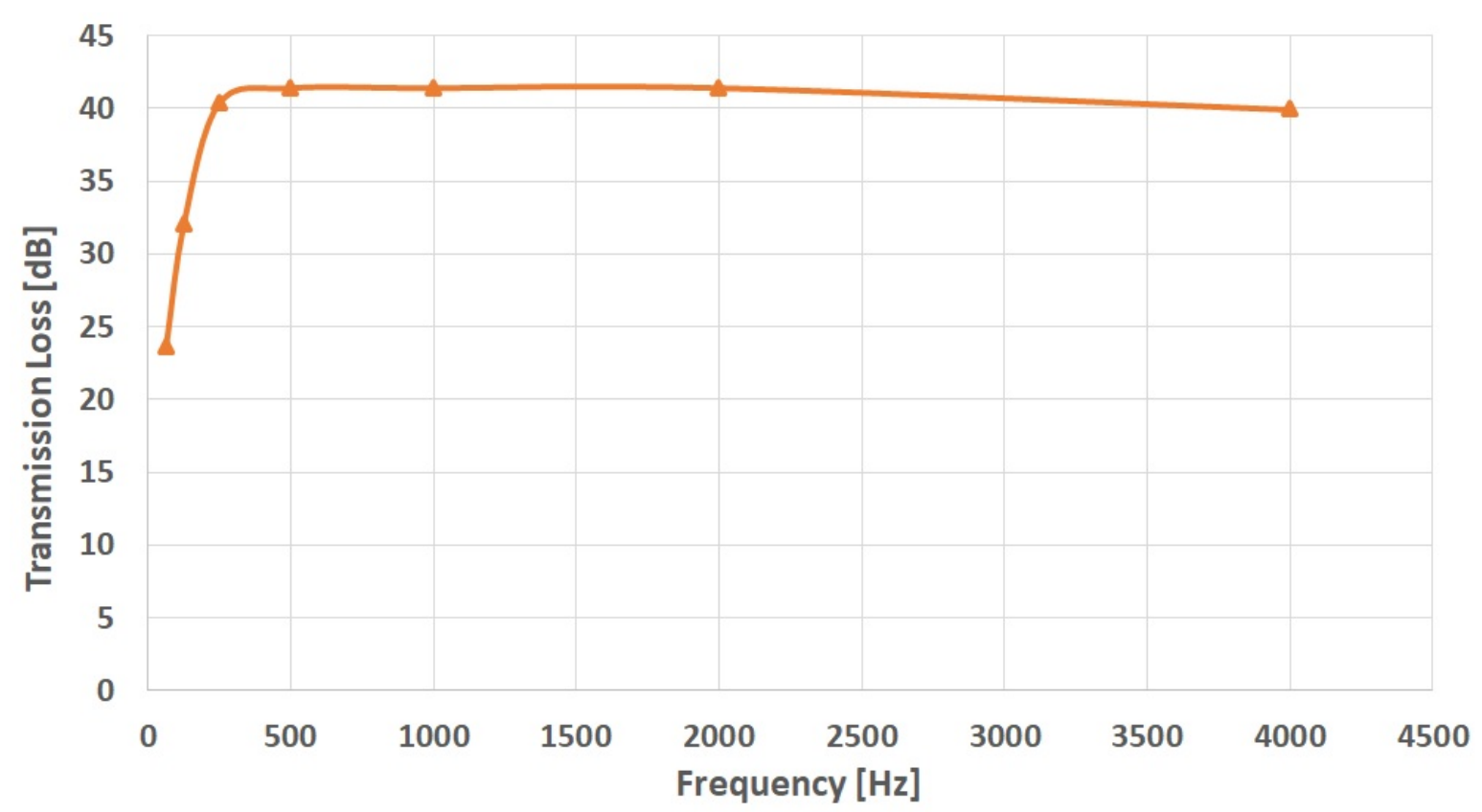

Figure 2.26: HSAWT two-pass plenum silencer design transmission loss performance predictions.

\subsubsection{Test Section Jet Collector}

An open jet collector system is a crucial component employed in many wind tunnels with open jet test sections [41 44, 69] as they are used to collect the flow from the nozzle jet orifice to the exhaust portion of the circuit. Crucial design parameters for the open jet test section in general include both the test section length (distance from nozzle outlet to collector opening), as well as the dimensions of the collector. The length of the test section must be minimized to reduce losses and the possible development of unsteady flow from the interactions of the shear layer with the jet core and surrounding air. It is also important to minimize the open jet length to ensure that the test subject (in this case the pressure fluctuation sensor array) is immersed within the potential core of the jet (region where the centreline mean velocity is roughly uniform and the boundary layer is relatively homogeneous). With the test section length governed as a fixed design constraint to house the previously designed 
test panel, the design of the collector dimensions are predicated on the dimensions of the nozzle orifice and the growth of the shear layer from the jet.

Generally speaking, the size of the collector opening should be selected to capture both the jet core and shear layer as smoothly as possible to avoid any degradation in test section flow quality. Abramovich [70] suggests that the collector should just capture the jet core mass flow equal to that emitted from the nozzle in order to avoid a longitudinal static pressure gradient in the usable test section area. Too large of a collector opening can cause significant entrainment and curvature of air streamlines leading to substantial recirculation flows, losses, and poor flow quality entering the exhaust portion of the wind tunnel [42, 71]. Too small a collector opening however will enable the jet shear layer to impinge and interact with the collector lips, which can produce upstream propagation of large test section pressure fluctuations triggering vortex shedding from the nozzle and jet oscillations [72]. Figure 2.27 outlines this 'feedback loop' which can cause significant edge tones and background noise contamination if there is any coupling between the large-scale coherent shed vortices with the resonant modes of the test chamber and duct work. This issue has both aerodynamic and aeroacoustic implications in terms of test section measurement quality.

Optimal design of the collector shape and inlet dimensions is usually difficult to conduct since there is a variety of testing configurations (flush flat panel, airfoil with large angle of attack, etc.) and nozzle orifice shapes (circular, rectangular, etc.) which all have implications on the collector design. An optimal design for one configuration may not prove to be beneficial for another, therefore compromises have to be made to ensure reasonable flow quality over the majority of the testing/flow conditions of interest. Final designs of collectors usually require direct scale model experiments or CFD to supplement rough design estimates from basic empirical shear layer behavior. 


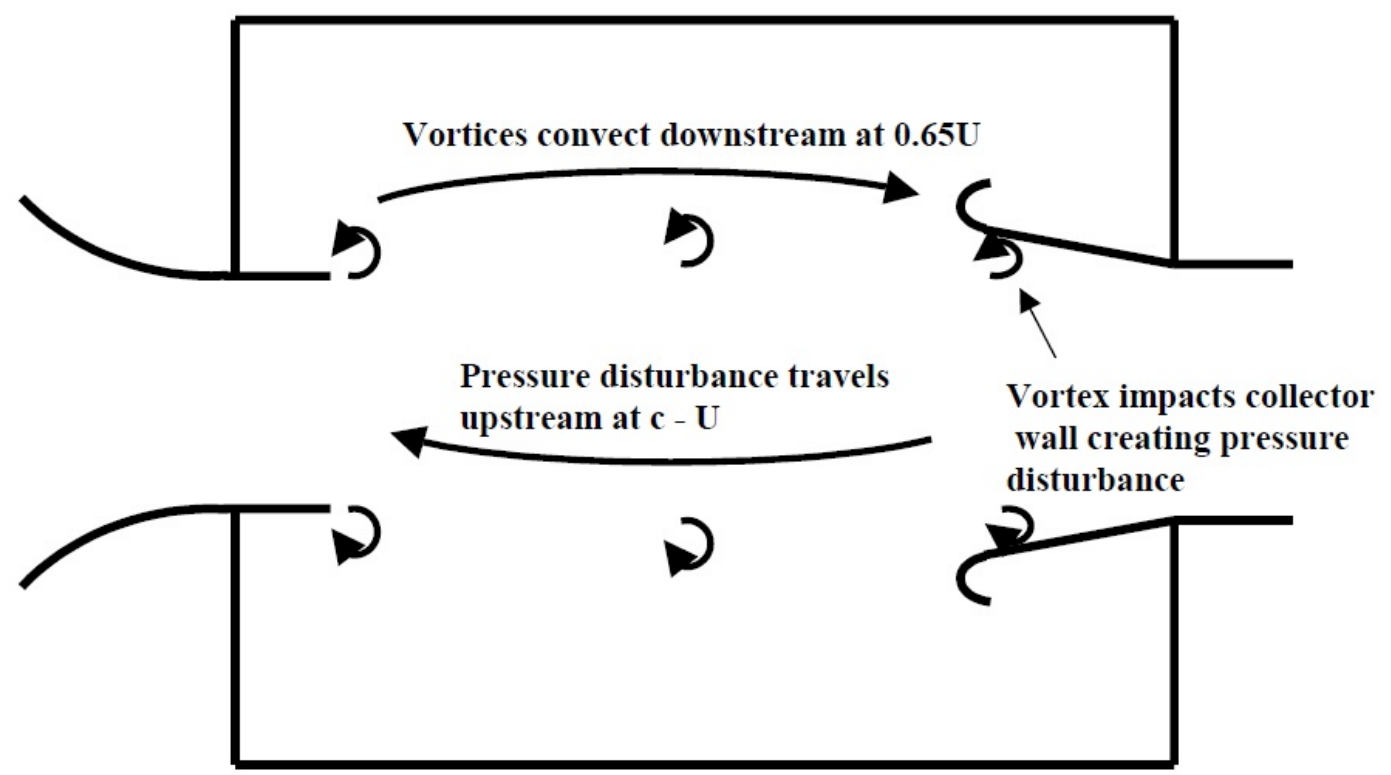

Figure 2.27: Diagram of general open-jet test section pressure fluctuation edge-tone feedback mechanism (taken from [72]).

The initial collector design for the HSAWT was obtained using similar considerations from the design of the NASA Langley open jet wind tunnel facility (presented in Figure 2.28] [42]. The collector mouth dimensions are designed to capture the jet from a rectangular nozzle exhaust over a flat test section floor with predicted shear layer growth angles of around $7^{\circ}$ in the spanwise direction and $1-2^{\circ}$ in the elevation plane. These shear layer growth behaviors were estimated based on classical empirical relationships developed by Abramovich [70] for a circular jet but modified to account for the increased jet spreading rate in the near flow field for a rectangular nozzle [73, 74]. The collector mouth is designed to have rounded inlet edges with relatively long straight walls and a smooth contraction to the collector throat; which is beneficial in terms of reducing test section turbulence levels and static pressure gradients [75. The collector throat should be on the order of the size of the nozzle to ensure proper flow quality to the exhaust diffuser [42], however restrictions in terms of the 


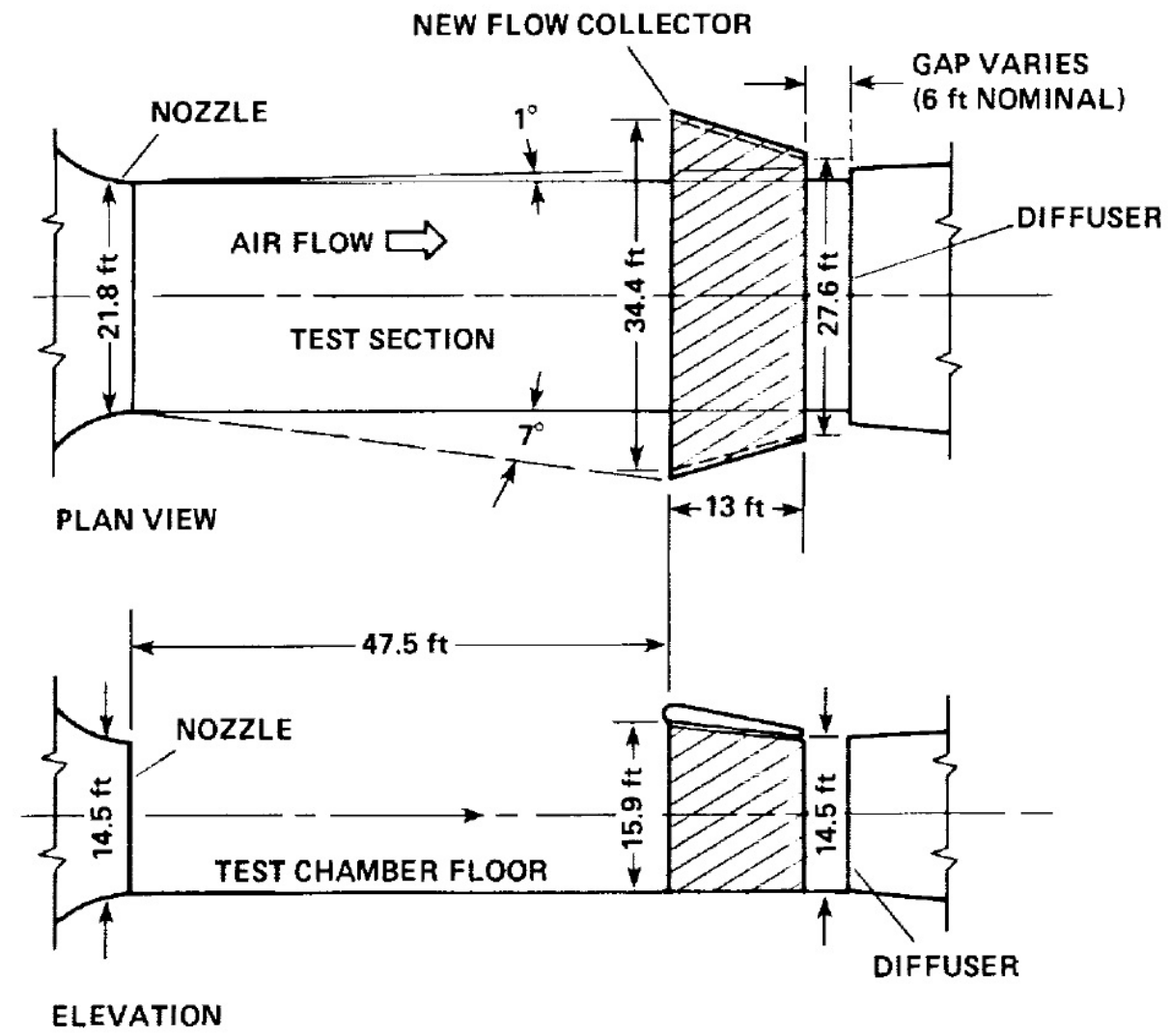

Figure 2.28: NASA Langley open jet wind tunnel test section schematic (taken from [42]).

downstream diffuser component design and available space for the exhaust system dictated the final dimensions for the collector contraction length and throat area.

A preliminary 3-D, steady-state, CFD analysis was performed to ensure that the initial design of the collector properly captured the shear layer and core of the jet. The entire chamber was modeled along with the nozzle outlet/geometry, test panel, and collector geometry up to the throat. The chamber walls were modeled without the wedges for simplicity; although the total chamber volume was reduced to account for the wedge volume displacement. All support structures and fittings were disregarded in the analysis. The ventilation holes and silencers were also neglected since modeling their pressure loss contributions for the mass flow entrainment was too complicated 
for this design analysis. Figure 2.29 contains the fluid domain model used for this study, which was meshed using roughly 4.5 million tetrahedral elements. The mesh area around the test section was refined using a body of influence and all surfaces exposed to flow contain a refined boundary layer mesh. All other boundary conditions are similar to the other component CFD analyses (refer to Appendix A for more information).

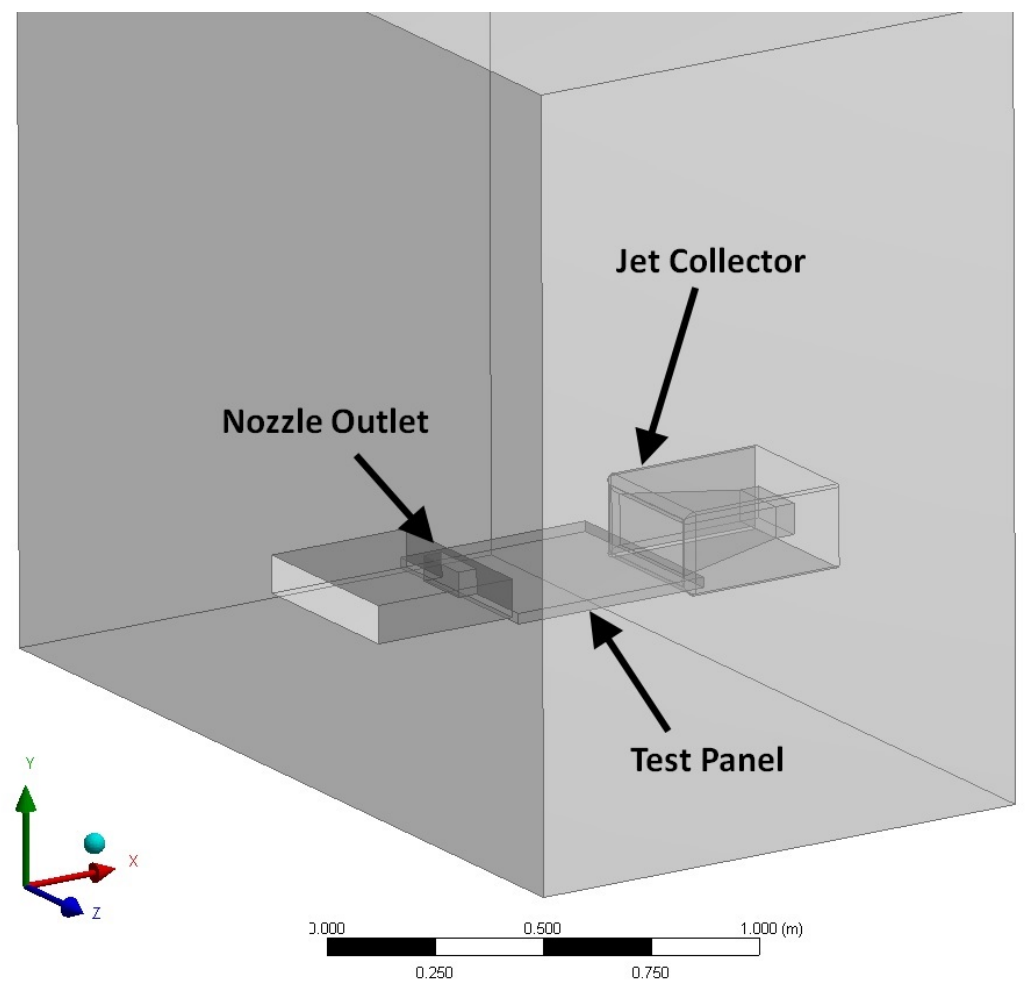

Figure 2.29: Modeled domain for test section/collector CFD analyses.

Figure 2.30 contains the CFD results of the preliminary collector configuration in terms of the spanwise and streamwise plane velocity vector fields. The plot of the spanwise field indicates that the selected dimensions for the mouth opening are too large for the size of the core jet and shear layer at that location due to the large areas of flow reversal near the lips. Furthermore, the streamwise plot displays a major stagnant area near the panel and the bottom collector lip. This indicates that the 
collector cannot be positioned along the centreline of the nozzle orifice and must be elevated slightly to account for the effects of the flat panel in the test section.

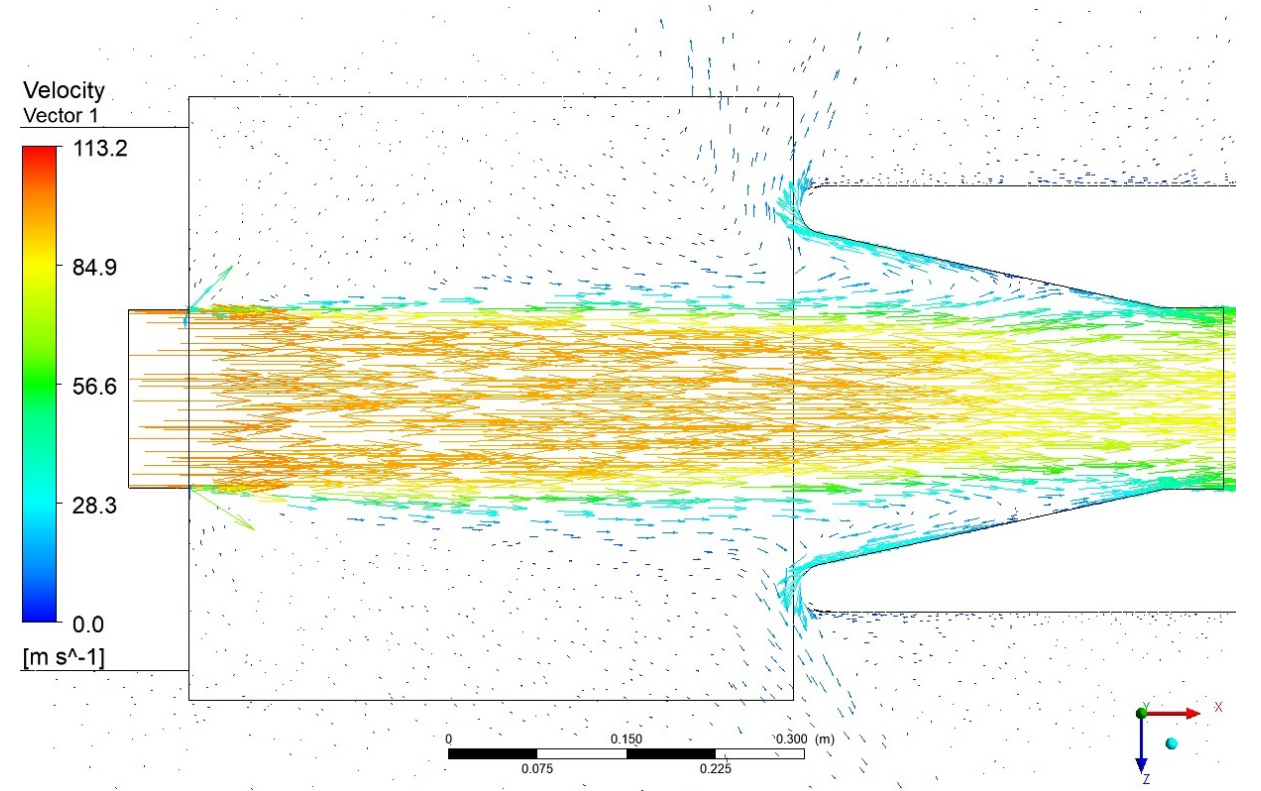

(a) Spanwise plane

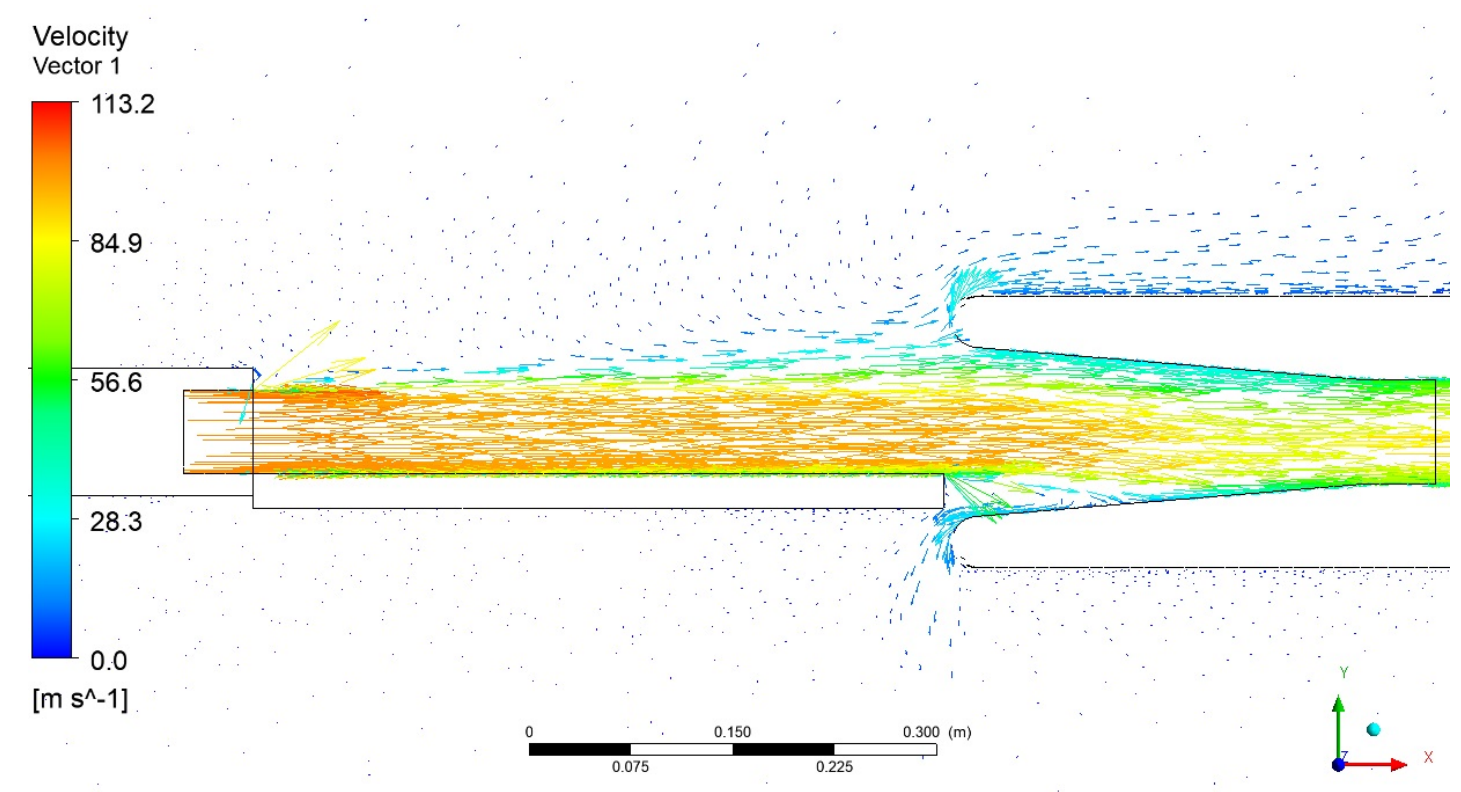

(b) Streamwise Plane

Figure 2.30: Velocity vector field plots - Preliminary collector CFD validation analysis. 
Figure 2.31 contains some flow streamline data, which includes the streamwise static pressure profile across the test panel and the spanwise profile of the outlet velocity at the throat of the collector. The static pressure profile indicates an acceptable ZMPG condition over the acrylic panel where the bulk of the pressure fluctuation sensor array is contained (0.197-0.323 $\mathrm{m}$ from the nozzle orifice). The outlet velocity profile however is severely non-uniform.
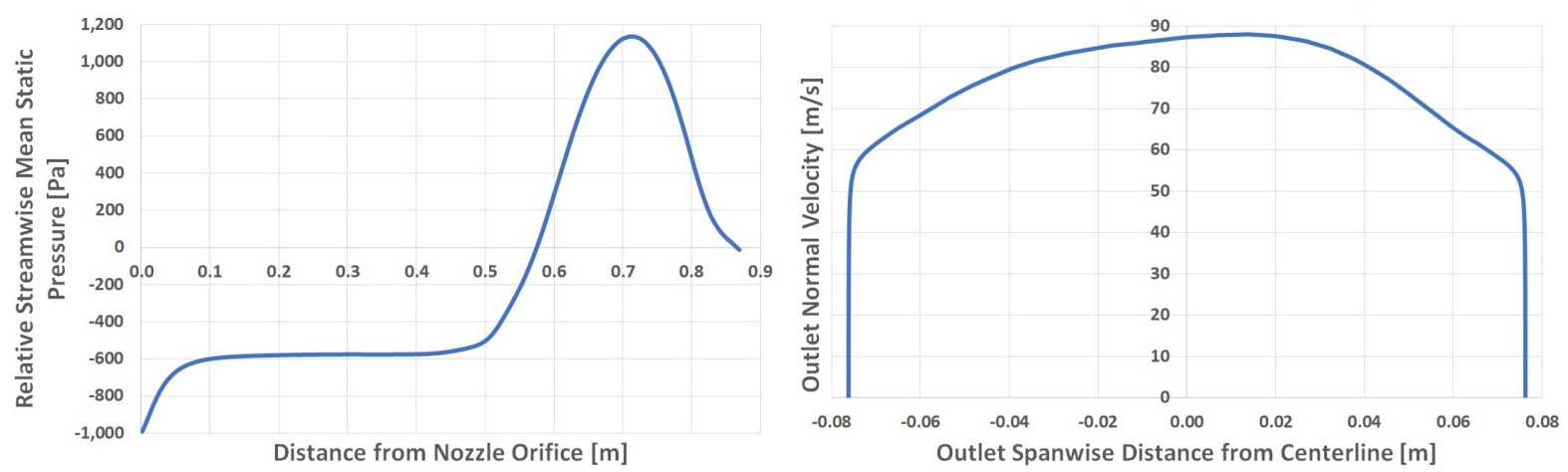

(a) Realtive test section centreline static pressure (b) Collector outlet spanwise plane velocity profile profile

Figure 2.31: Test chamber flow analysis plots - Preliminary collector CFD validation analysis.

Various CFD analyses were performed to try to determine the optimum dimensions from the preliminary design. The size of the collector mouth was reduced until the major regions of flow reversal were attenuated. Figure 2.32 contains the spanwise and streamwise plane velocity vector field results from the CFD analysis of the final selected collector design. The results from the final design show substantial improvement in terms of the flow quality in both plane direction from reducing the size of the mouth and shifting the collector centreline slightly above the plane of the nozzle orifice. The spanwise velocity field still shows slight stagnant flow areas just inside of the lips, however, there are no signs of flow reversal. 


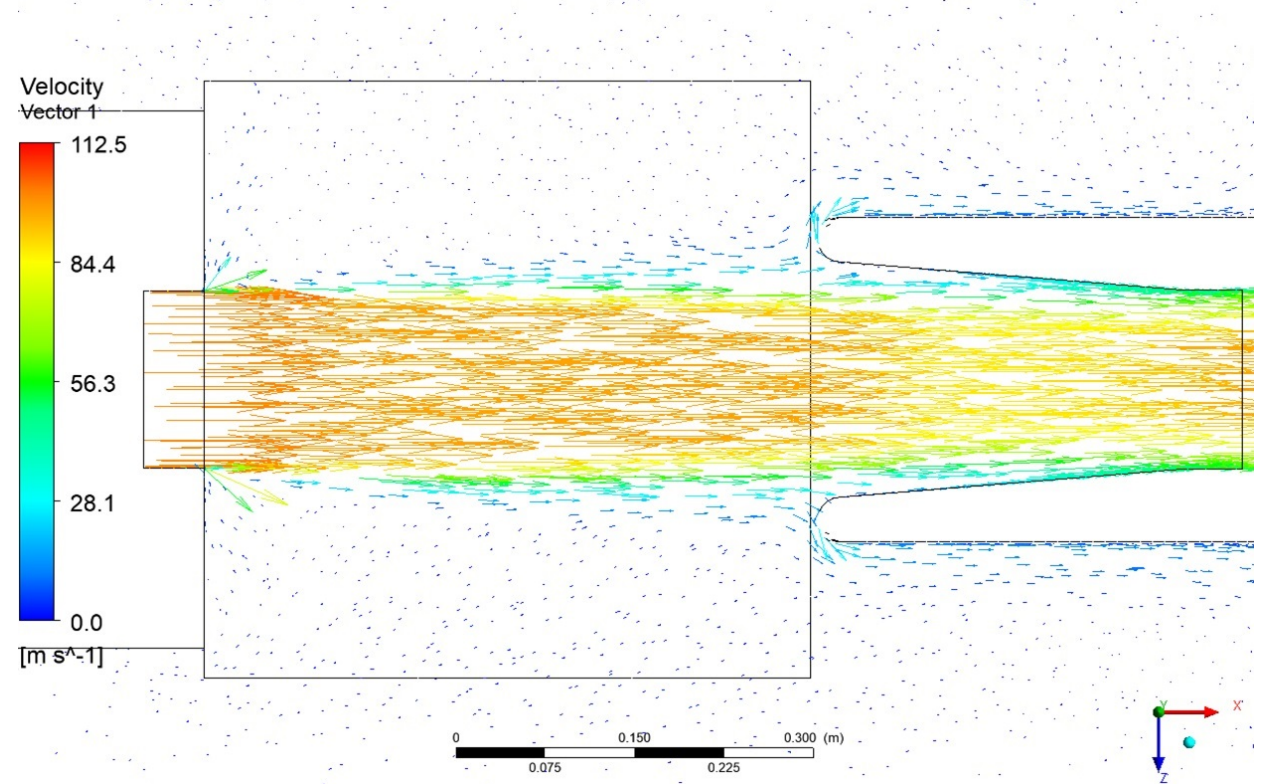

(a) Spanwise plane

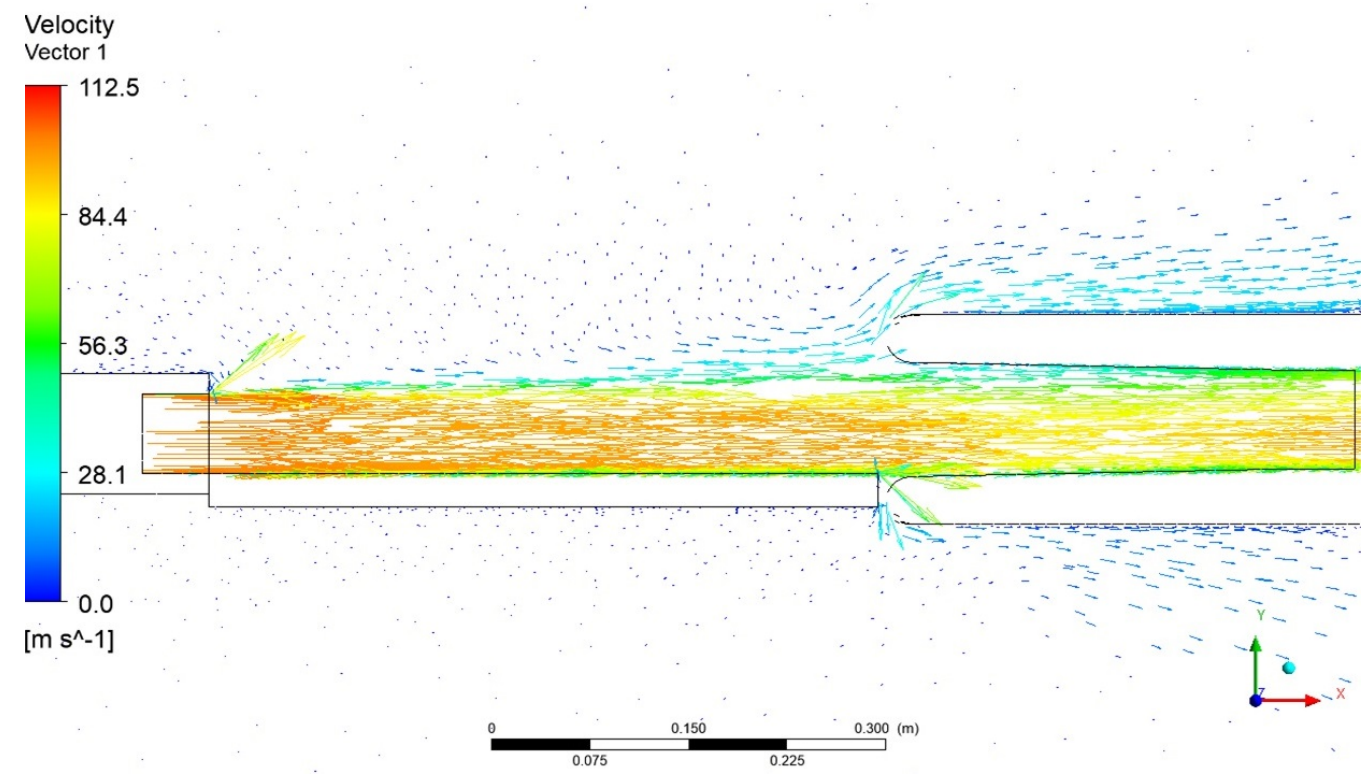

(b) Streamwise Plane

Figure 2.32: Velocity vector field plots - Final collector CFD validation analysis.

Figure 2.33 contains similar streamline flow data regarding the new final design configuration for comparison. The results show improvements in terms of the pressure losses across the test section, as well as a more consistent velocity profile at the outlet 
of the collector. It is important to note however that these CFD results cannot completely replace the need for experimental verification of the flow quality and zeromean streamwise pressure gradient conditions within the test section. Chapter 7 discusses this matter in further detail.
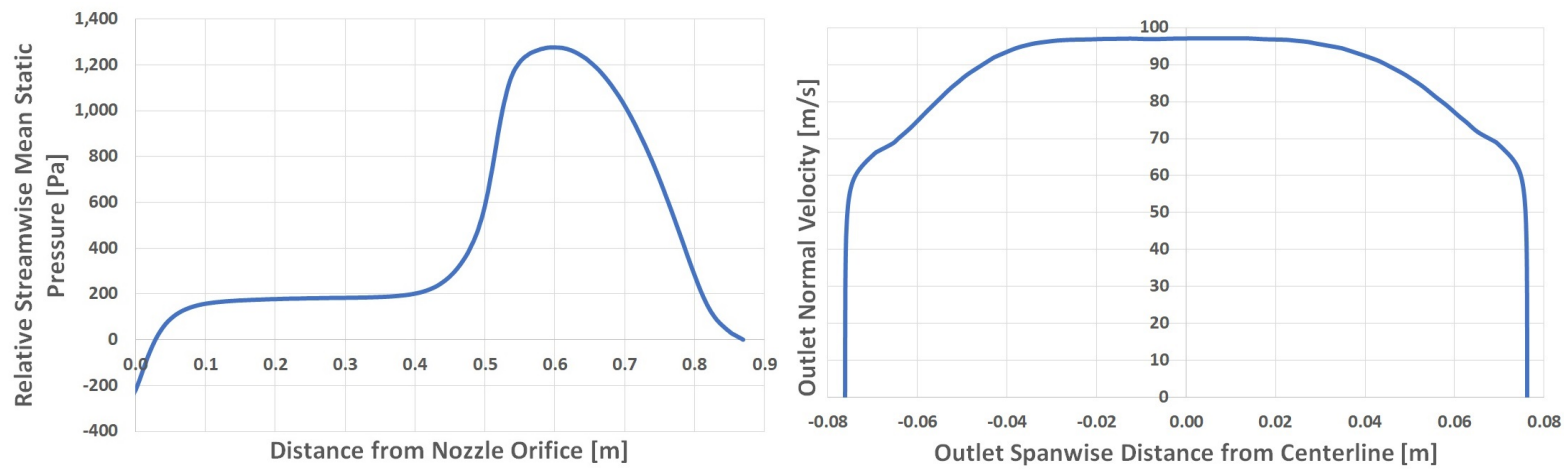

(a) Realtive test section centreline static pressure (b) Collector outlet spanwise plane velocity profile profile

Figure 2.33: Test chamber flow analysis plots - Final collector CFD validation analysis.

A schematic of the final fixed design for the HSAWT jet collector is presented in Figure 2.34 along with relevant dimensions. The centreline of the collector is positioned roughly $1.27 \mathrm{~cm}$ above the centreline of the nozzle orifice. 


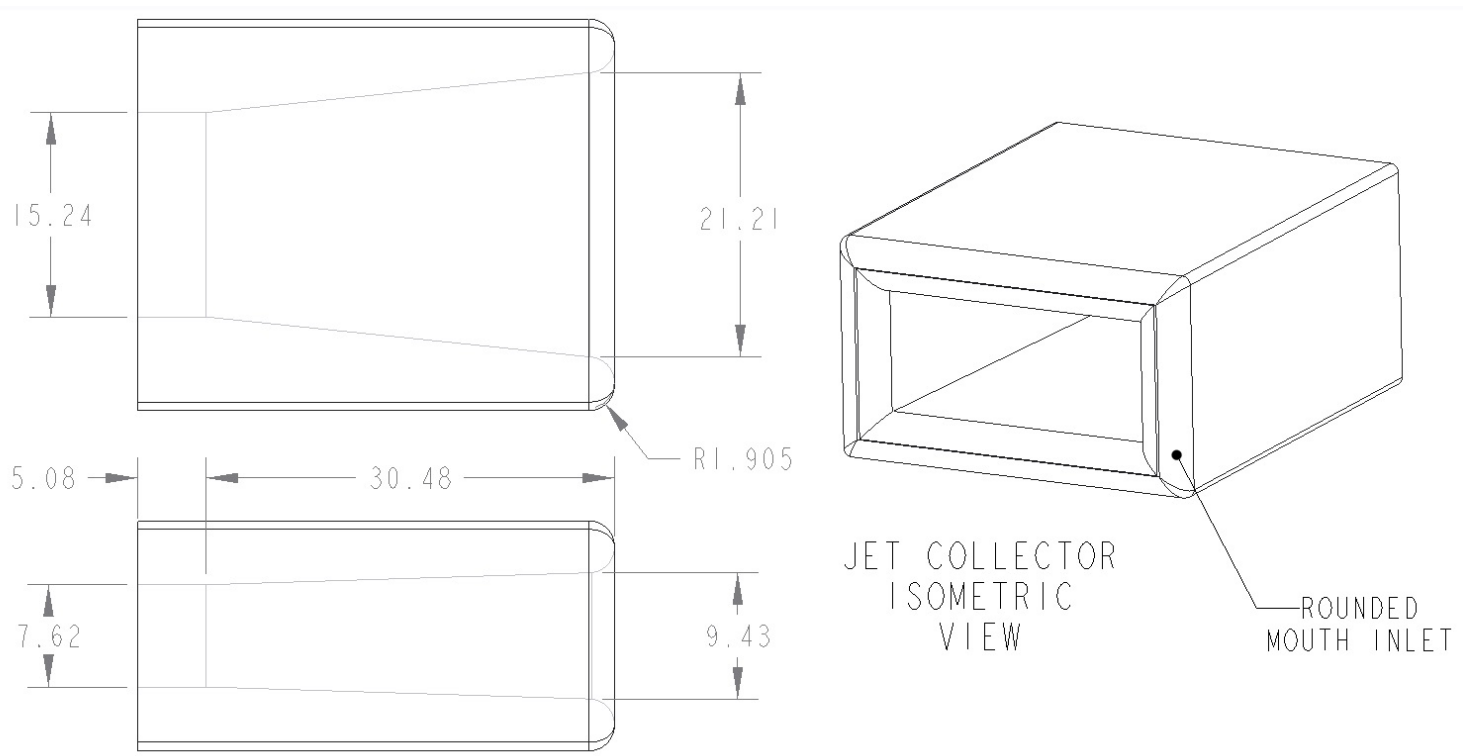

Figure 2.34: Jet collector shape with selected dimensions (all dimensions in $\mathrm{cm}$ ).

\subsubsection{Collector and Vertical Exhaust Diffusers}

The purpose of a diffuser in an exhaust system is intended to reduce the flow velocity downstream of the test section by a gradual duct area increase to recover some of the static pressure head of the flow [76, 77]. An efficient diffuser is designed to convert the maximum possible percentage of kinetic energy into pressure through smooth expansion with minimal losses due to friction and flow separation.

The use of two-dimensional flat-walled diffusers (expansion in one plane) was selected as the base configuration of the design of both the collector and vertical exhaust diffusers due to their simplicity in manufacturing and the imposed dimensional constraints of the test chamber and facility layout. 2D diffusers also have the benefit of allowing for larger expansion angles for a given area ratio compared to a conical diffuser [44]. The generalized geometrical definition of a $2 \mathrm{D}$ diffuser is presented in Figure 2.35. 


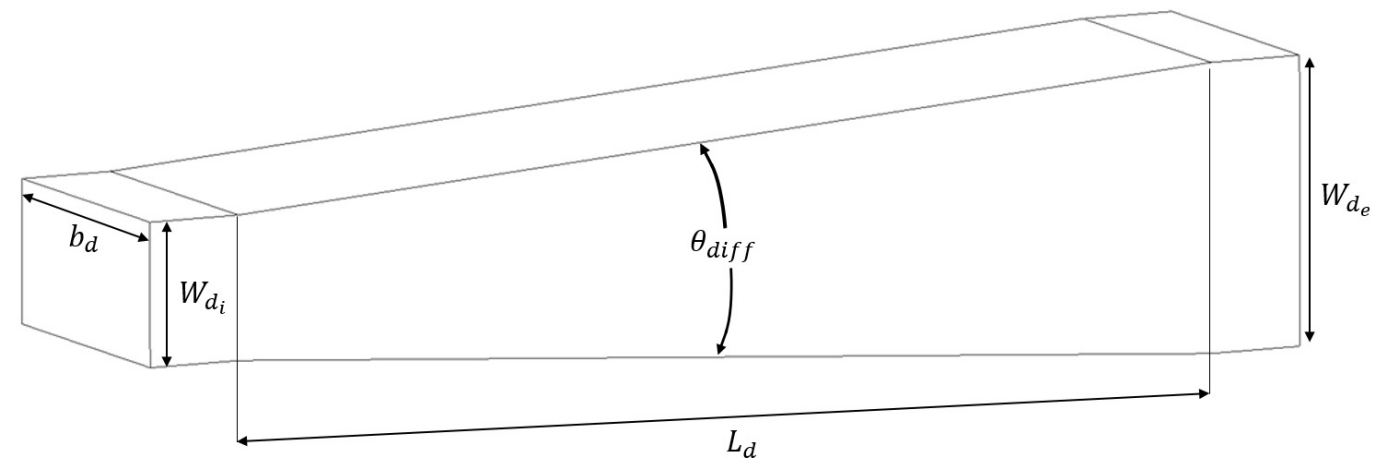

Figure 2.35: General two-dimensional flat-walled diffuser geometry.

The performance of a diffuser is heavily influenced by its wall-to-wall divergence angle, $\theta_{\text {diff }}$, and its non-dimensional length, $L_{d} / W_{d_{i}}$ [76]. These two parameters ultimately dictate whether the expansion is smooth enough to avoid flow separation and increased pressure losses (diffuser stall). Operating an unstalled diffuser is the goal in terms of selecting the proper dimensions given the design constraints. The selection of the design parameters and dimensions for the collector and vertical exhaust diffusers is provided in the drawing schematic shown in Figure 2.36.

The chosen dimensions were first based on given spatial constraints of the chamber/facility and the dimensions of the upstream components. The dimensions of the collector exhaust diffuser inlet were therefore fixed at 15.24 by $7.62 \mathrm{~cm}$. The $90^{\circ}$ corner section in the exhaust system connecting the two diffuser sections must also contain a square cross-section, which fixes the dimensions of the collector exhaust diffuser outlet to 15.24 by $15.24 \mathrm{~cm}$. This also relates to the dimensions of the vertical exhaust diffuser inlet. The remaining dimensions were selected based on spatial constraints as well as to optimize the performance of the diffuser while avoiding the possibility of flow separation. Data published by Fox and Kline [78] (which are summarized into diffuser design charts by Runstadler [79] and Blevins [77]), were used 

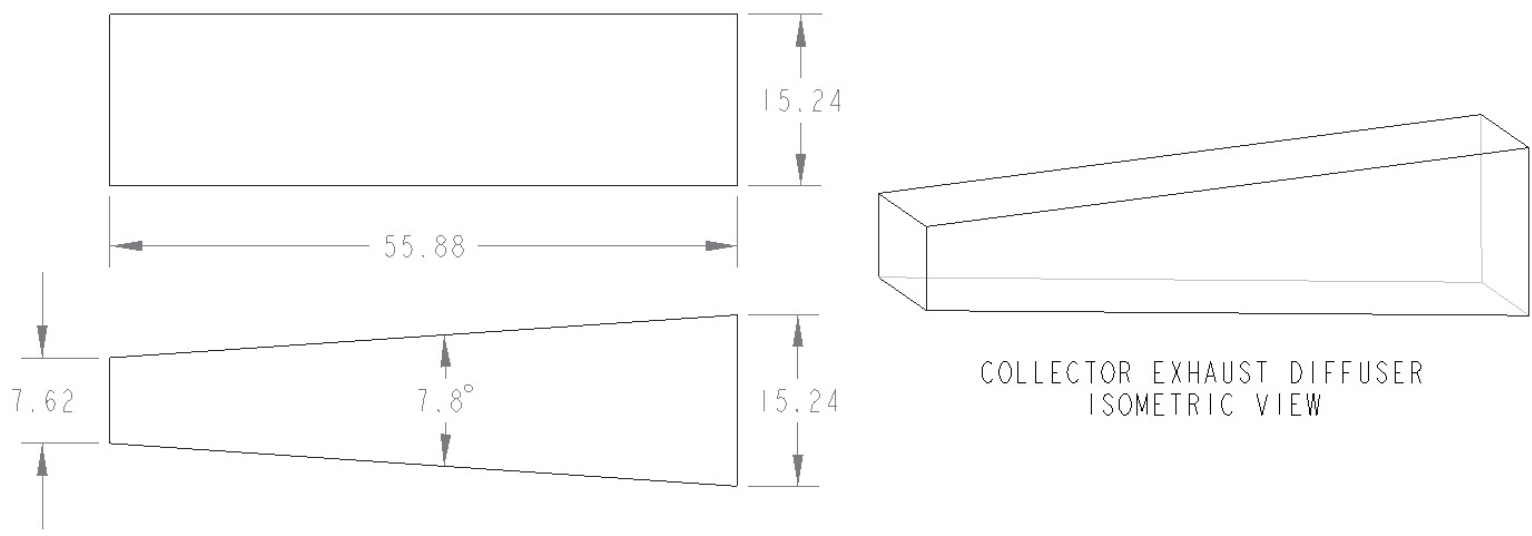

(a) Collector exhaust diffuser

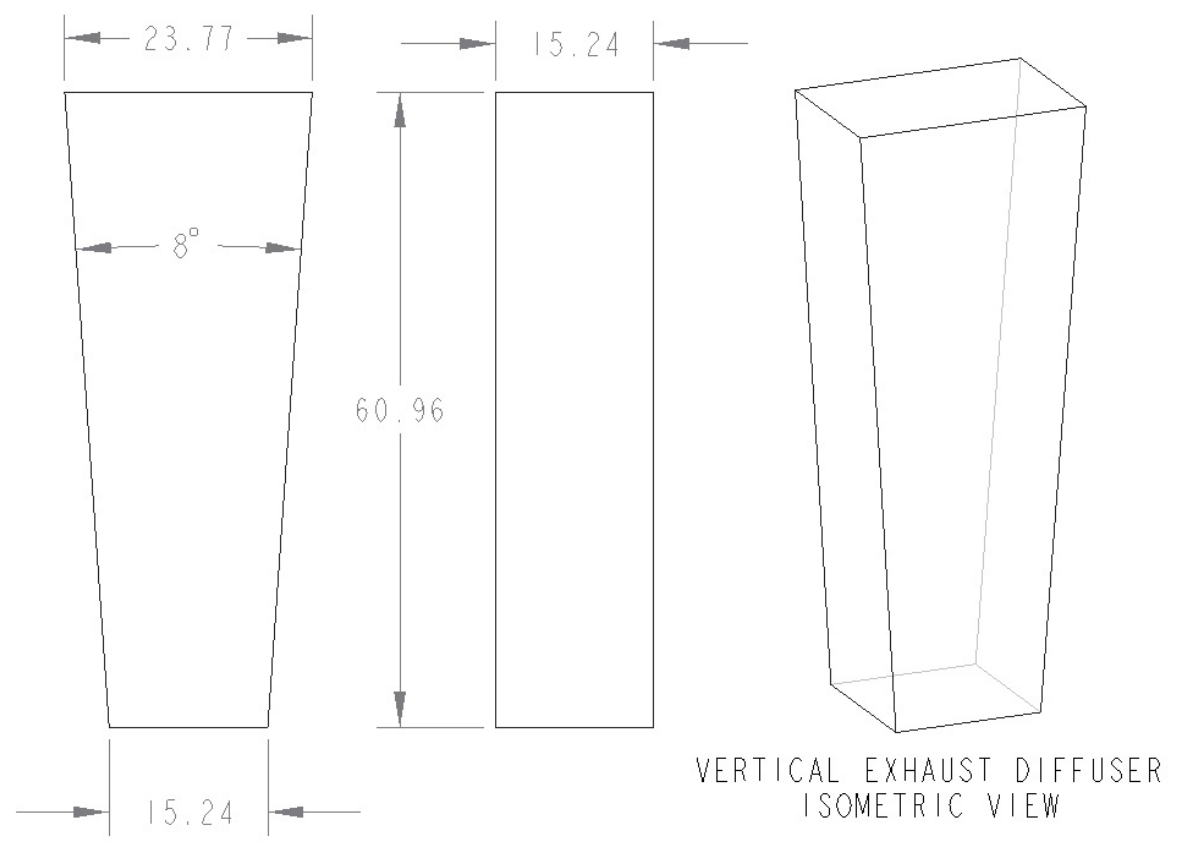

(b) Vertical exhaust diffuser

Figure 2.36: HSAWT exhaust system diffuser designs: internal duct shape and definition (all dimensions in $\mathrm{cm}$ ).

to ensure that the chosen dimensions of diffusers did not lead to operation in any unwanted stall condition. Figure 2.37 contains this summarized data in the form of design charts. 


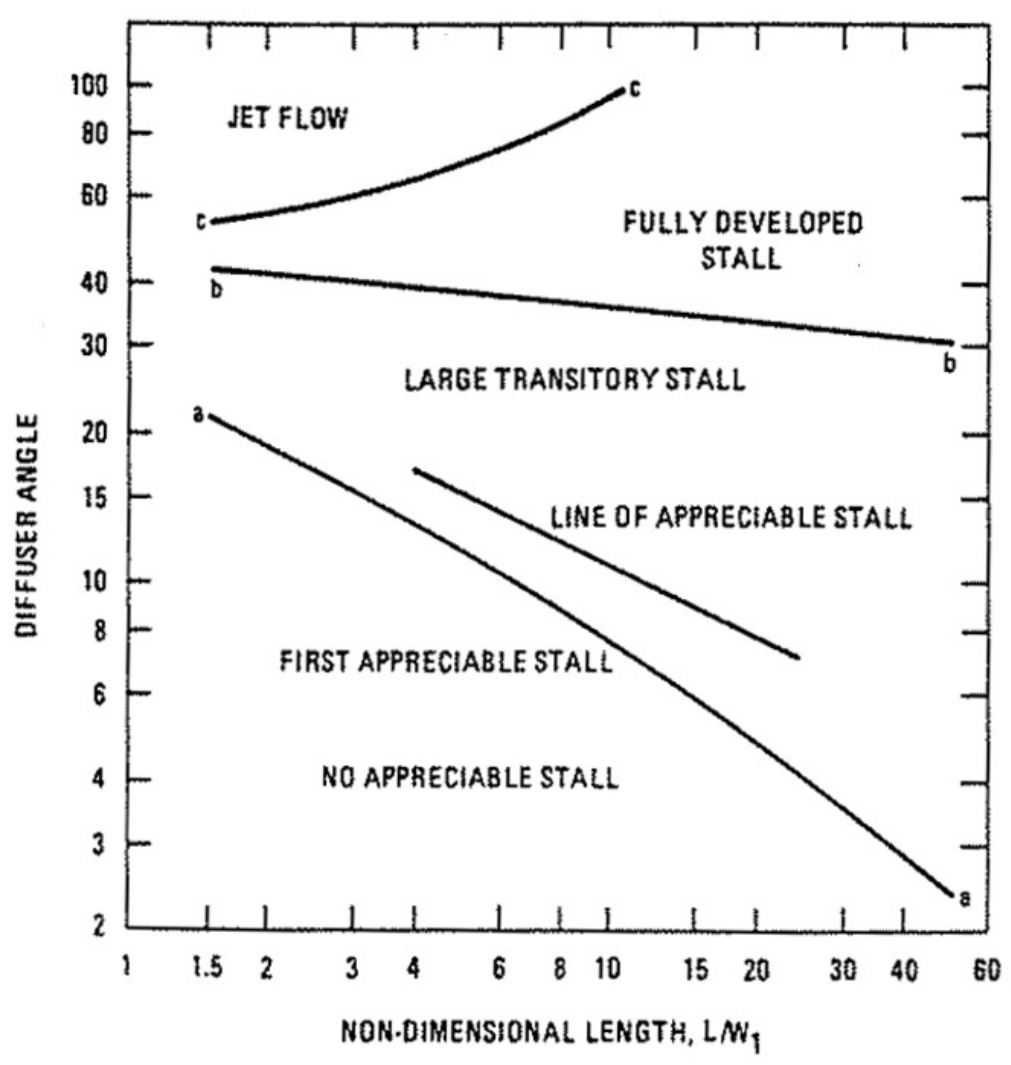

(a) General diffuser stability map

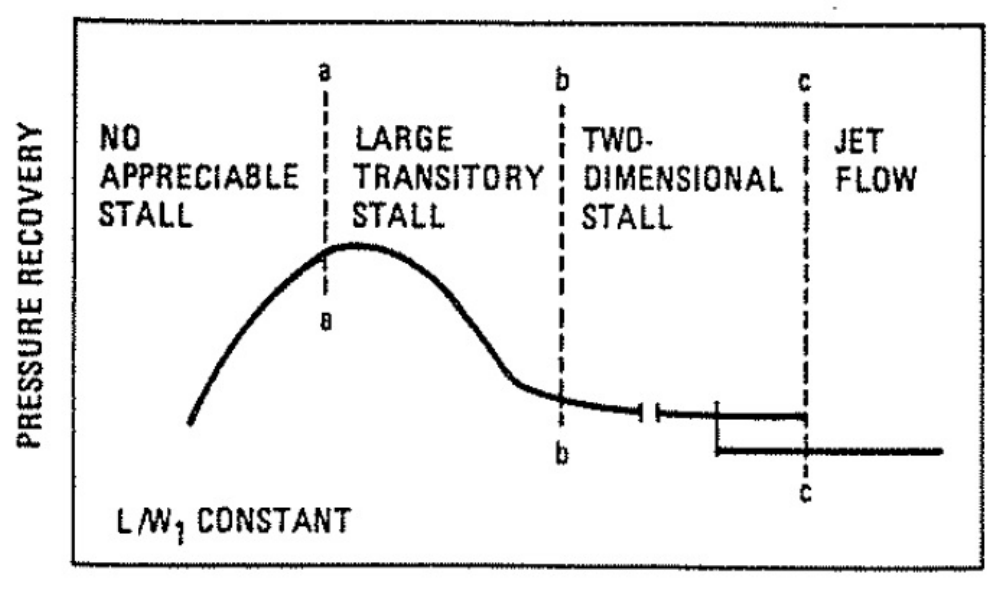

DIFFUSER ANGLE

(b) General diffuser performance map

Figure 2.37: Fox and Kline [78] two-dimensional flat-walled diffuser data (design charts taken from [77, 79]). 
The first chart displayed in Figure 2.37 contains a stability map, which predicts the expected diffuser flow pattern or region of operation for a given diffuser design based on the divergence angle, $\theta_{\text {diff }}$, and the non-dimensional length, $L_{d} / W_{d_{i}}$. The idea is to select the dimensions of the diffuser so as to stay in the region outlined by no appreciable stall (below the line of first appreciable stall). Theoretically, the region of best diffuser performance occurs just over the line of first appreciable stall, as shown by the diffuser performance map in Figure 2.37; however, a conservative approach was taken to ensure that both diffuser designs would not operate with any flow separation.

A CFD analysis was also performed to further validate the designs in terms of avoiding any flow separation. The results of the divergence plane streamwise velocity contour plots from the CFD analysis are shown in Figure 2.38 for both diffuser designs. Similar solver initial and boundary conditions were used for this analysis as was done for the transition channel computations. Both computational meshes were constructed of approximately 6 million unstructured hexahedral elements. Both plots indicate that the flow velocity is reduced without any significant regions of separation along the walls. 

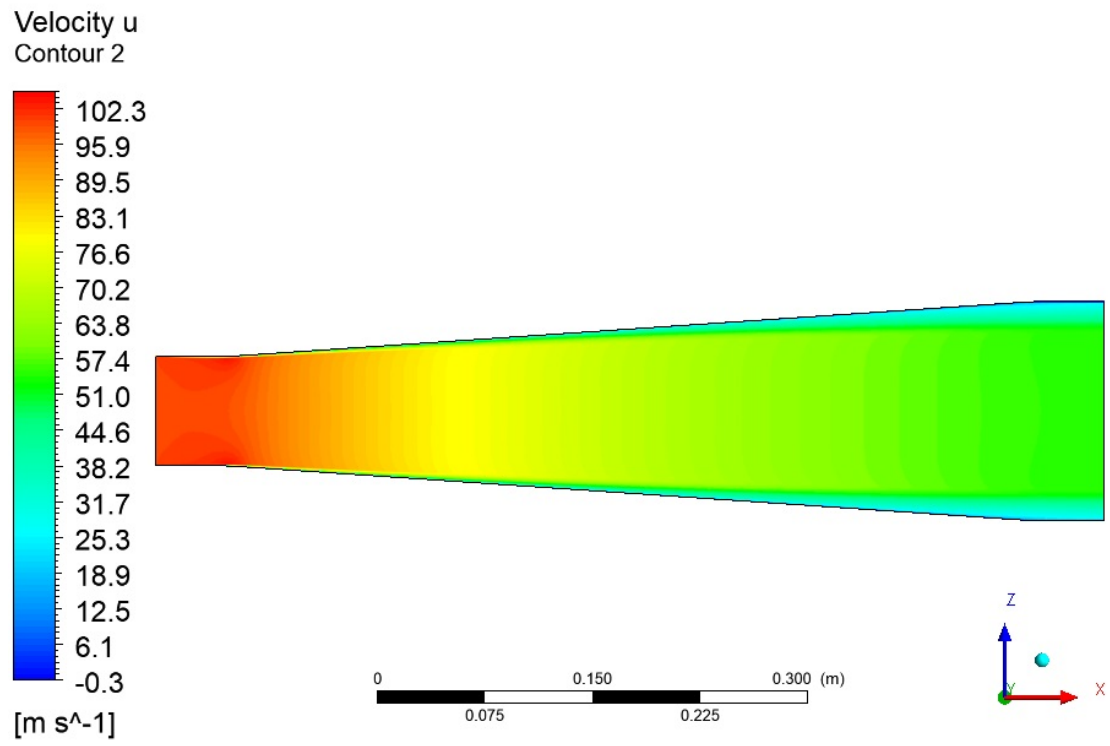

(a) Collector Exhaust Diffuser

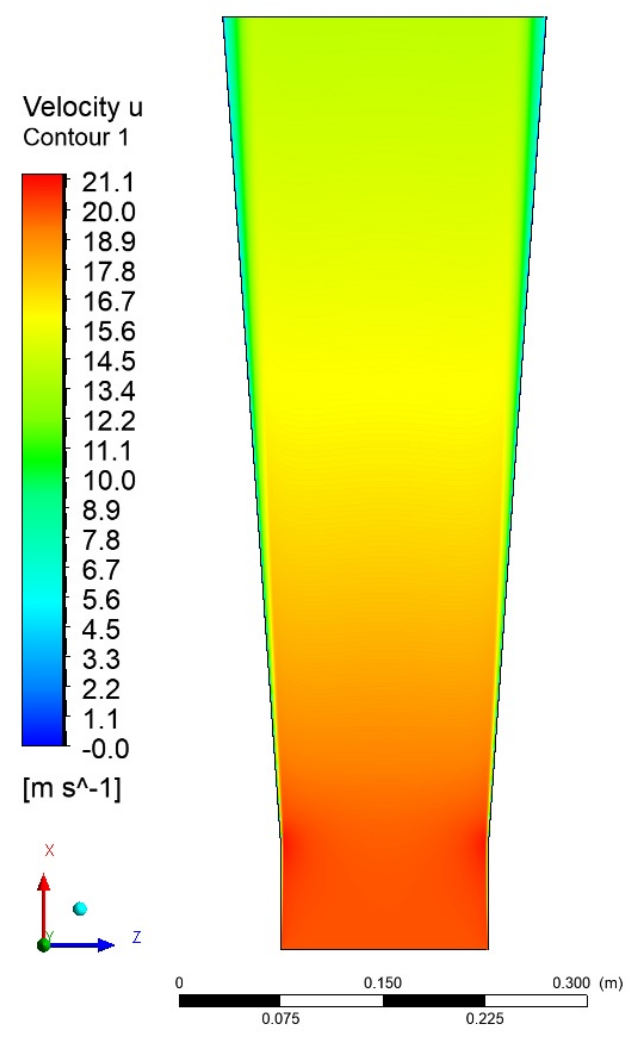

(b) Vertical Exhaust Diffuser

Figure 2.38: Divergence plane streamwise velocity contour plots - Diffuser channel CFD validation analysis. 


\subsubsection{Exhaust Corner Transition and Flow Turning Vanes}

The exhaust system corner is designed to provide a smooth $90^{\circ}$ vertical flow transition between the collector exhaust diffuser and the vertical exhaust diffuser. The corner is designed with a constant square duct area matching the dimensions of the collector exhaust diffuser outlet $(15.24 \times 15.24 \mathrm{~cm})$. The abrupt corner elbow design was selected to transition the flow vertically in as short of a distance as possible. The corner is therefore fitted internally with turning vanes, as well as corner fillets with a radius equal to that of the turning vanes, to ensure that minimal flow separation and exit flow non-uniformity is achieved. An internal schematic of the exhaust corner is shown in Figure 2.39.
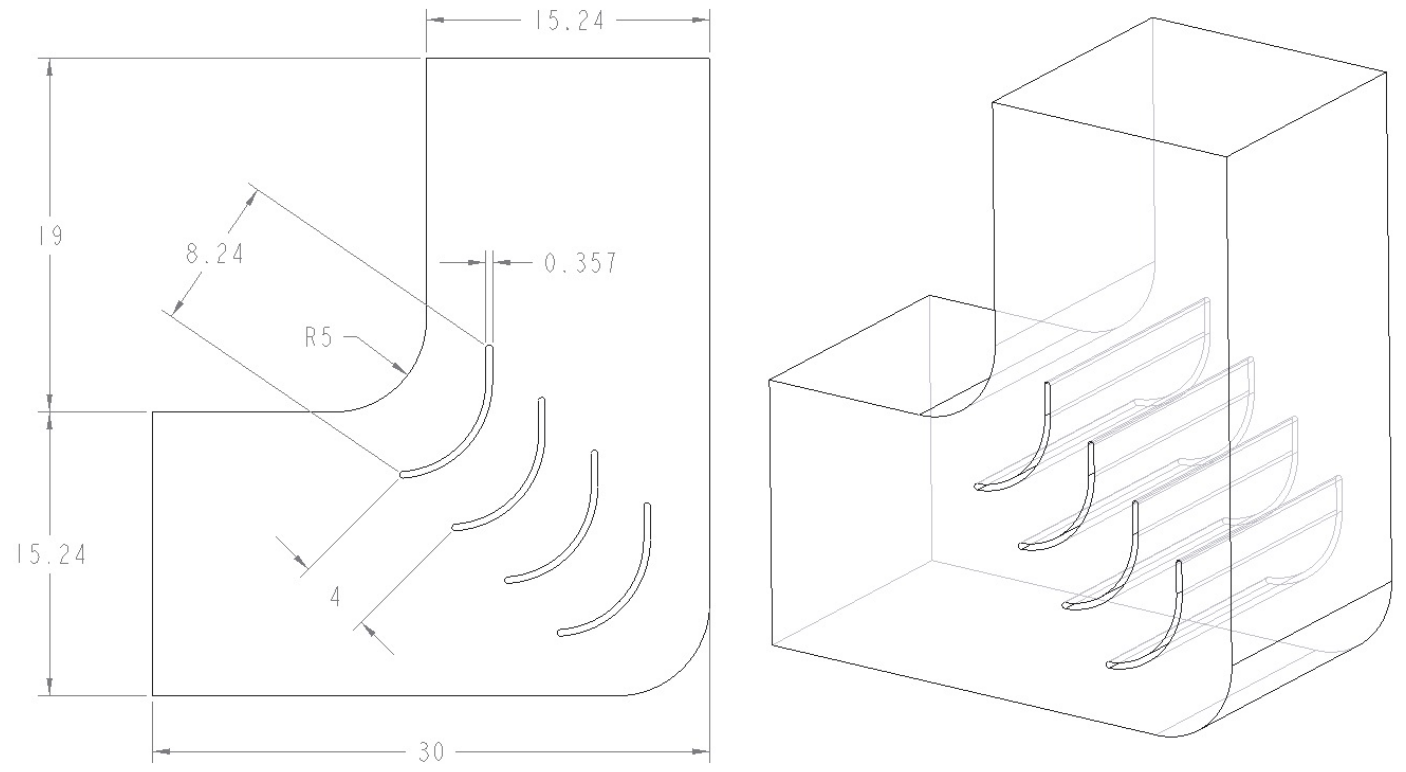

Figure 2.39: Internal schematic of exhaust corner (all dimensions in $\mathrm{cm}$ ).

The two main approaches to the design of turning vanes either involve the use of thin sheet metal vanes, which are formed to $90^{\circ}$ circular arcs with straight trailing edge extensions, or the use of thick airfoil profiled vanes (refer to Figure 2.40 for a 


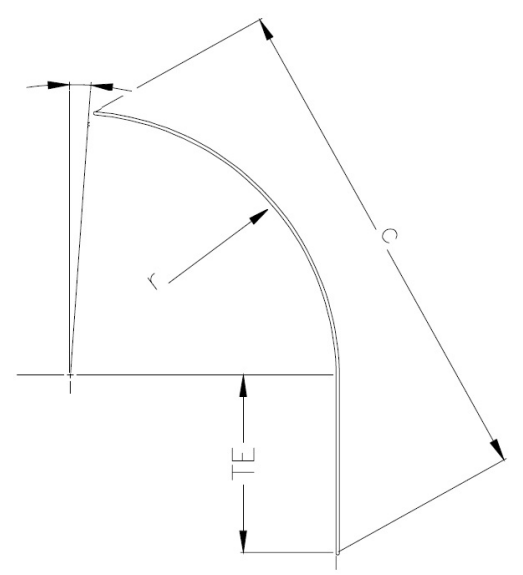

(a) Thin circular arc profile vane (adapted from [80])

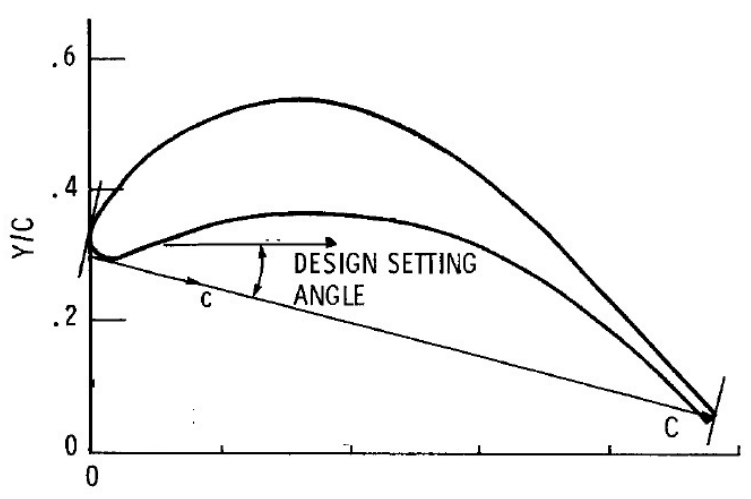

(b) Thick airfoil profile vane (adapted from [81])

Figure 2.40: Comparison of general turning vane designs.

visual comparison).

Although optimally designed thick airfoil vanes have been shown to reduce skin friction and total losses across a corner in comparison with thin vanes [81, the thin vane design approach was selected due to ease of manufacturing and installation. The radius of the vanes and corner fillet was determined based on scaled comparisons of the ratio of the vane chord to hydraulic diameter of the corner inlet. All other dimensional parameters are based on a scaled design outlined by Rozell [82, which is a standardized design used by the American Society of Heating, Refrigeration, and Air-Conditioning Engineers (ASHRAE). The positive angle of incidence of $2^{\circ}$ is implemented to help prevent separation along the suction side of the vane [80]. Figure 2.41 shows the designed turning vane profile.

With the selected spacing-to-chord ratio based from the outlined design by Rozell [82], 4 or 5 vanes can be placed in the vane rack with the available space in the corner channel. A 2-D CFD analysis was conducted to compare the two configurations based on outlet flow quality. A 2-D mesh consisting of 2.6 million elements, extruded 


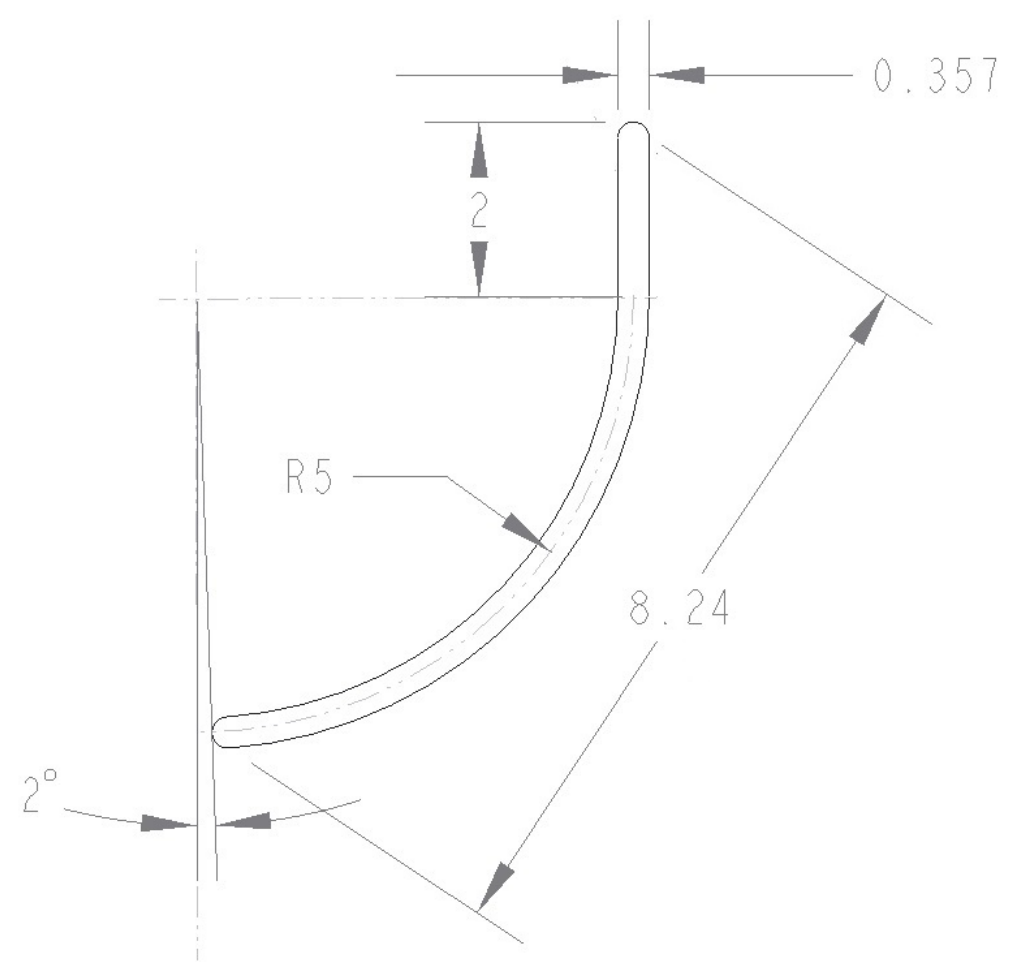

Figure 2.41: Turning vane design profile (all dimensions in $\mathrm{cm}$ ).

3 elements thick for implementation in CFX, was used for both simulations. Both of the plane ends were modeled using a symmetry boundary condition and similar constant velocity inlet and pressure outlet boundary conditions were used as in the other component simulations. Results were taken at the plane containing the central nodes of the extruded mesh. Further information is contained in Appendix A.

Figure 2.42 contains the velocity magnitude contour comparison between both vane configurations. The 4-vane configuration seems to show a distinct stagnant flow region in the far corner of the bend, which is slightly relieved when 5 vanes are implemented. When examining the outlet velocity profile comparison contained in Figure 2.43, the 5-vane configuration does show a slight improvement in terms of outlet flow uniformity, however this is quite minor and does so at the expense of an added vane and pressure losses. The 4-vane configuration was ultimately selected for the turning 


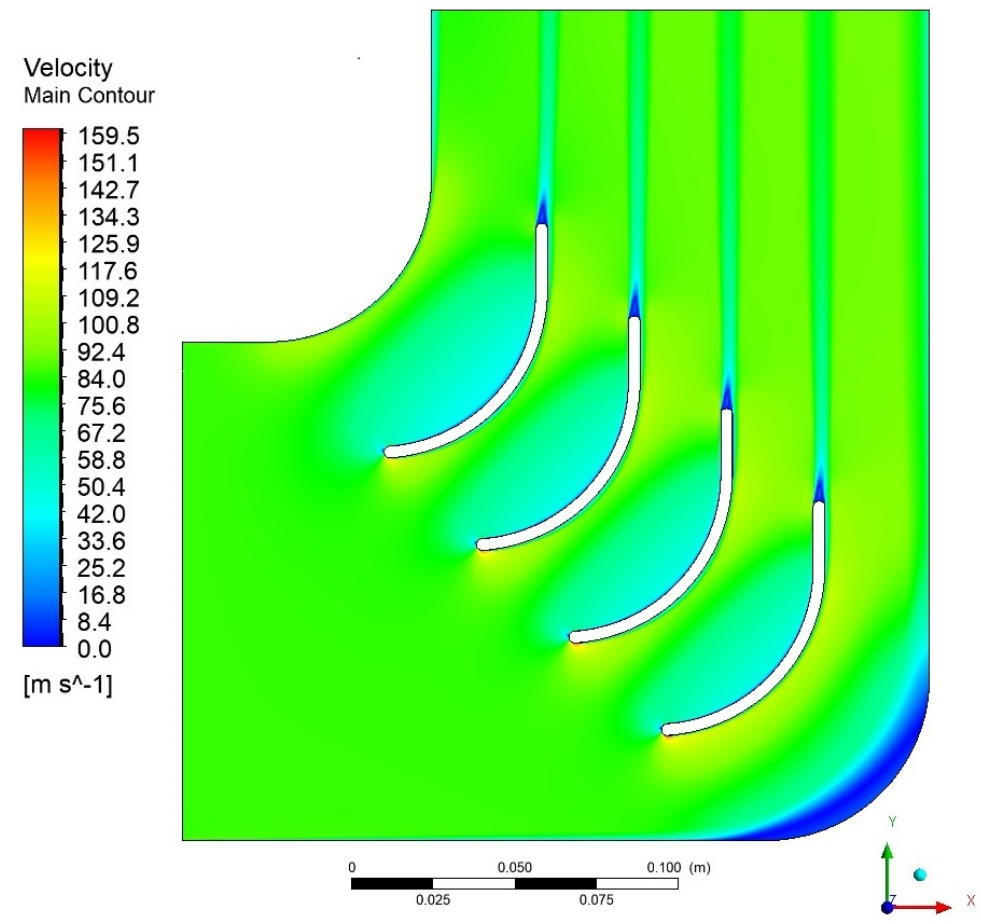

(a) 4 vane configuration

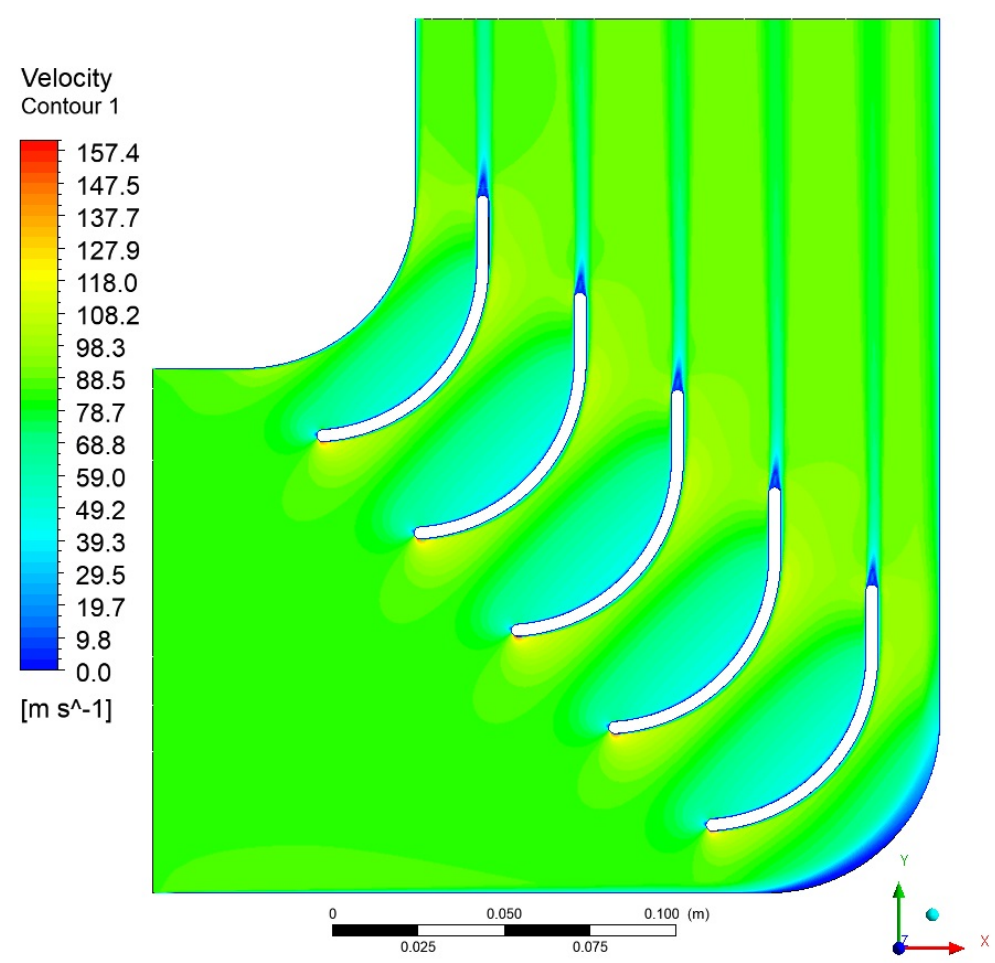

(b) 5 vane configuration

Figure 2.42: Plane velocity magnitude contour plots - Turning vane CFD validation analysis. 
vane rack due to minor improvements seen from the addition of an extra vane in which the benefit may be offset by the increased costs in manufacturing/installation and pressure losses. The turning vane rack is also designed to be removable which can allow for future optimized improvements.

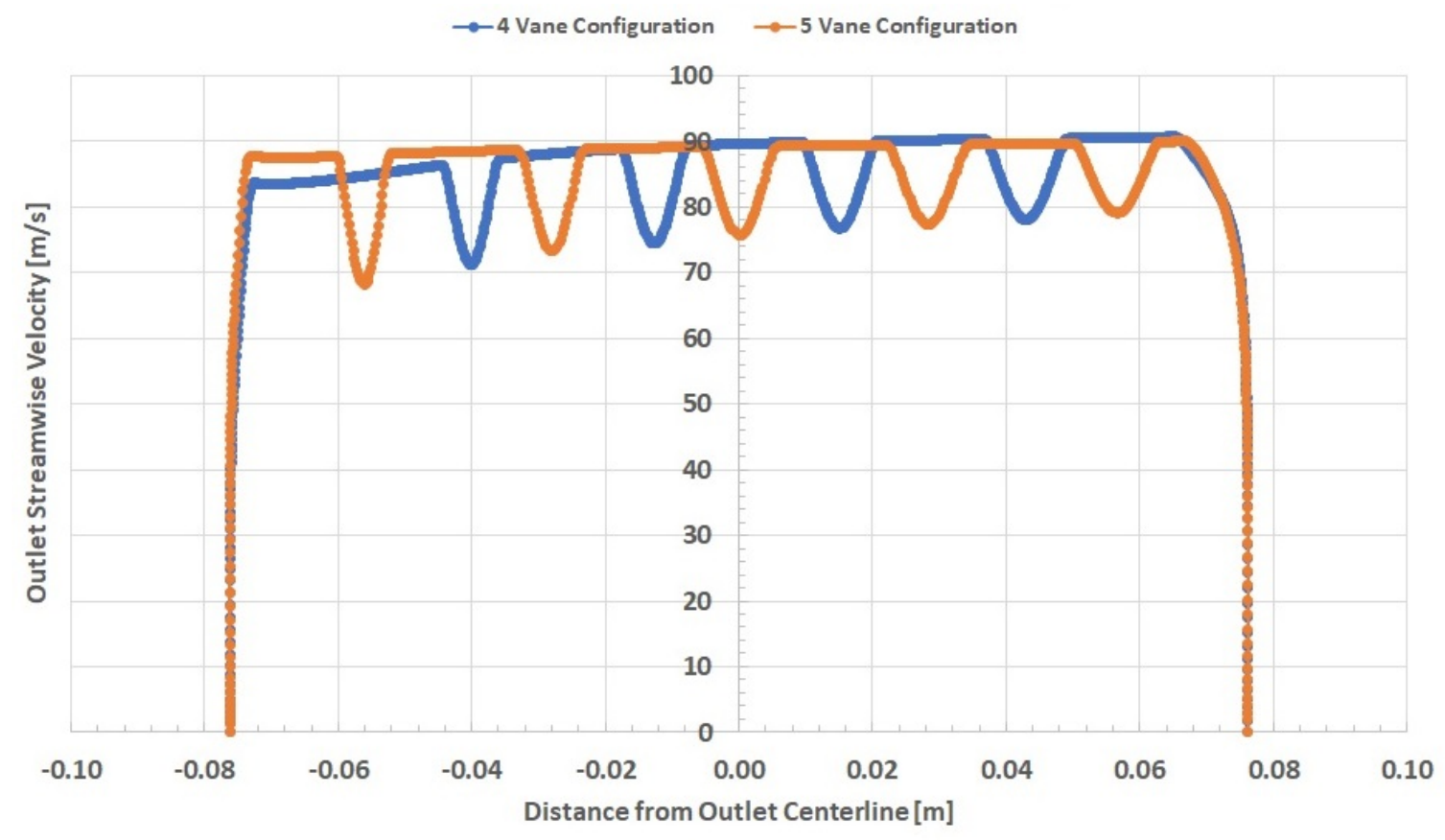

Figure 2.43: Corner Outlet centreline Velocity Profile - Turning vane CFD validation analysis. 


\section{Chapter 3}

\section{Fabrication of Wind Tunnel Components}

This chapter provides descriptions on the methods used to fabricate the component designs outlined in Chapter 2. Figures detailing how the components interface with each other are also provided. The acoustic treatments performed on some of the chamber interior components is described as well.

\subsection{Anechoic Chamber}

The anechoic chamber was fabricated and installed by Eckel Industries and TRA Acoustics. Figure 3.1 contains photos showing the assembly of the chamber. The chamber floor assembly was first constructed and fit with four 2000 lbs casters. The wall panels were installed on the chamber base and connected together with structural beams. Additional beams run along the top of the chamber for the installation of the ceiling panels. The acoustic wedges are installed within the chamber on fixed metal tracks. Figure 3.2 shows the completed chamber with the removable floor wedges in

place. The centre set of floor wedges were eventually removed for the placement of the test section components. 


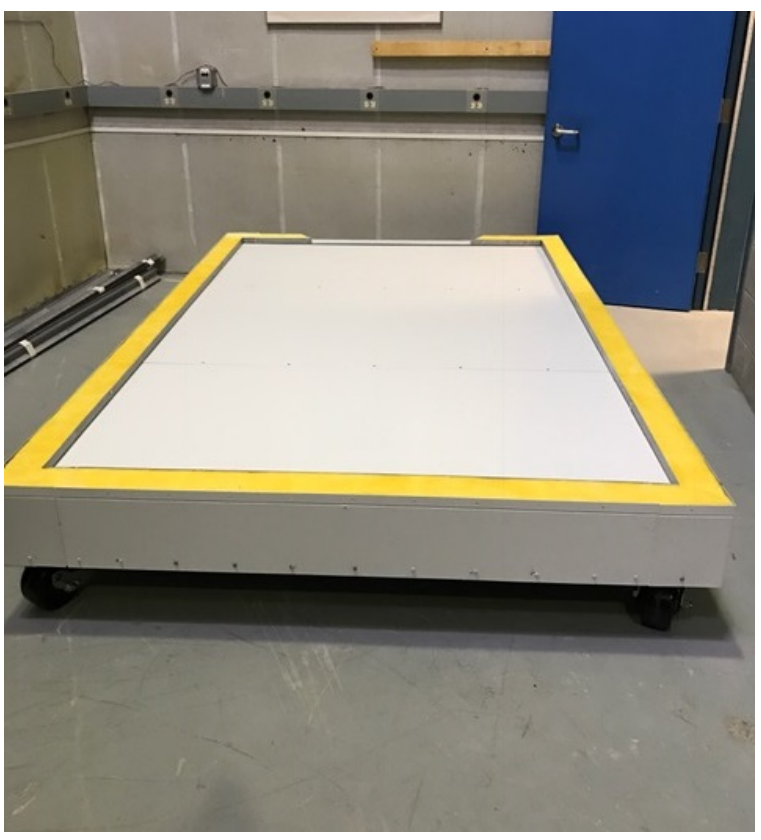

(a) Chamber floor

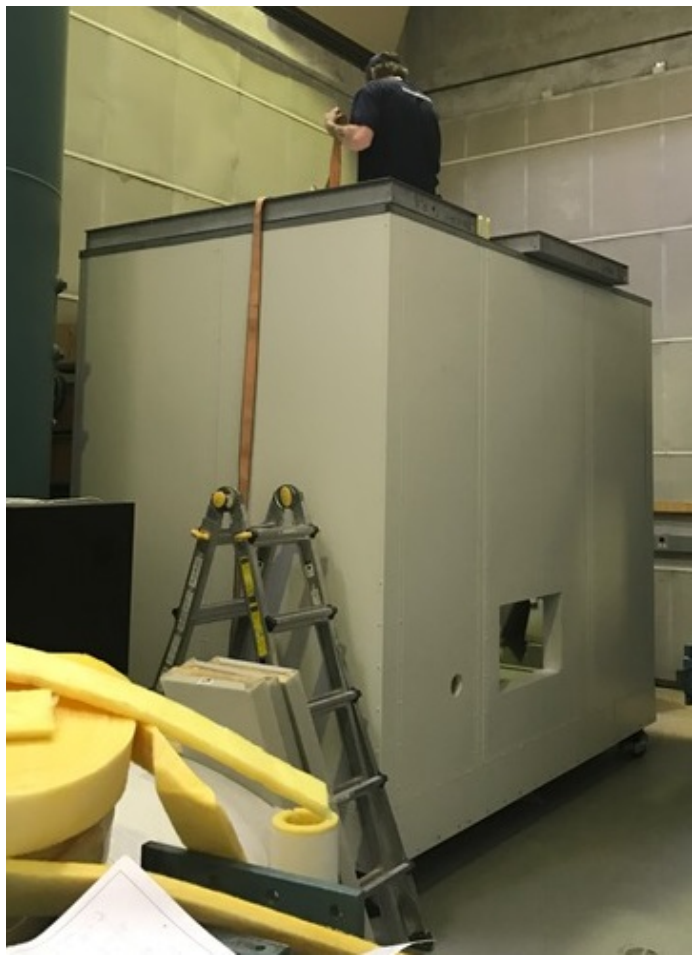

(c) Chamber ceiling

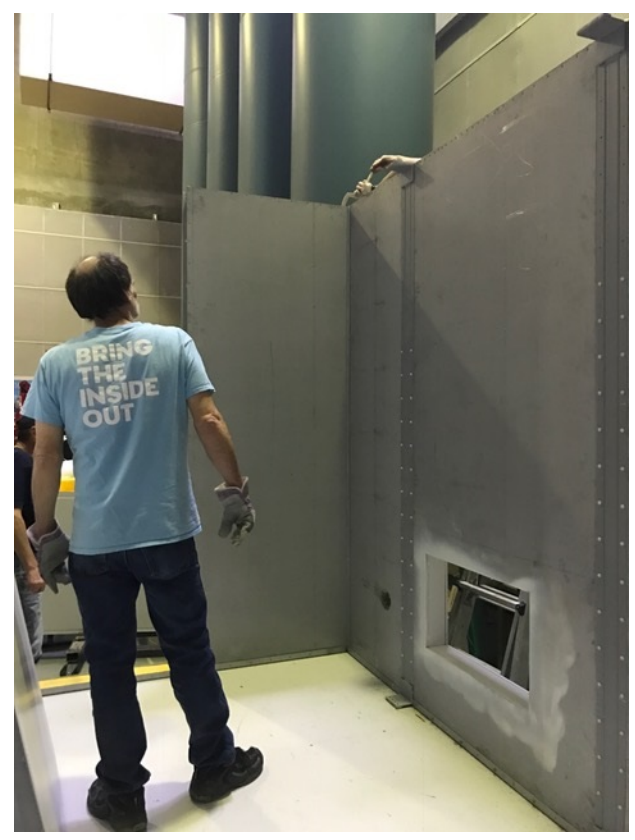

(b) Chamber walls

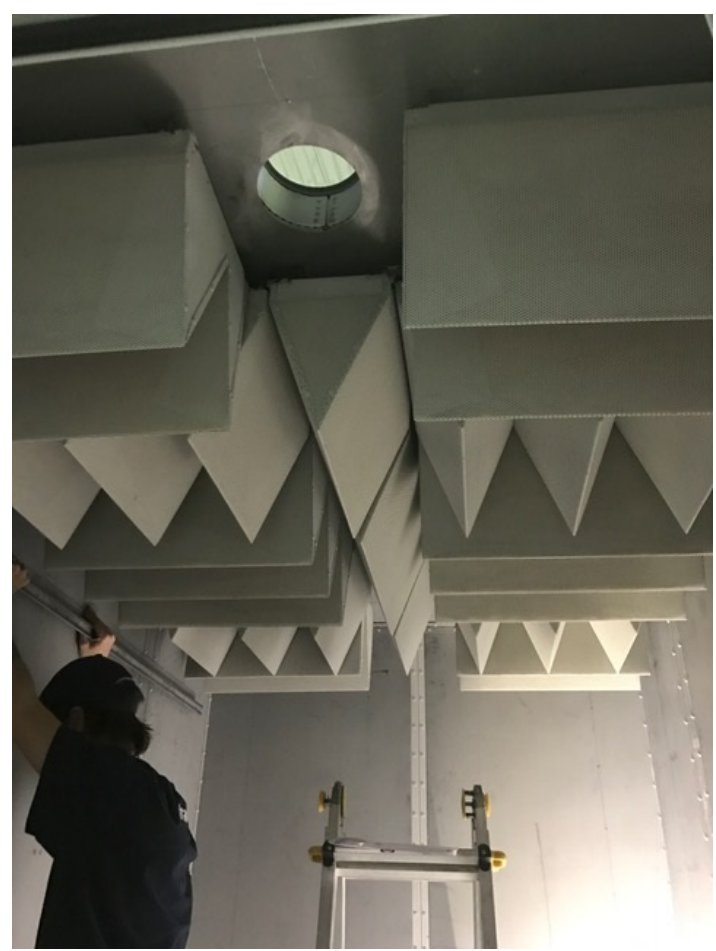

(d) Chamber acoustic wedges

Figure 3.1: Assembly of anechoic chamber. 


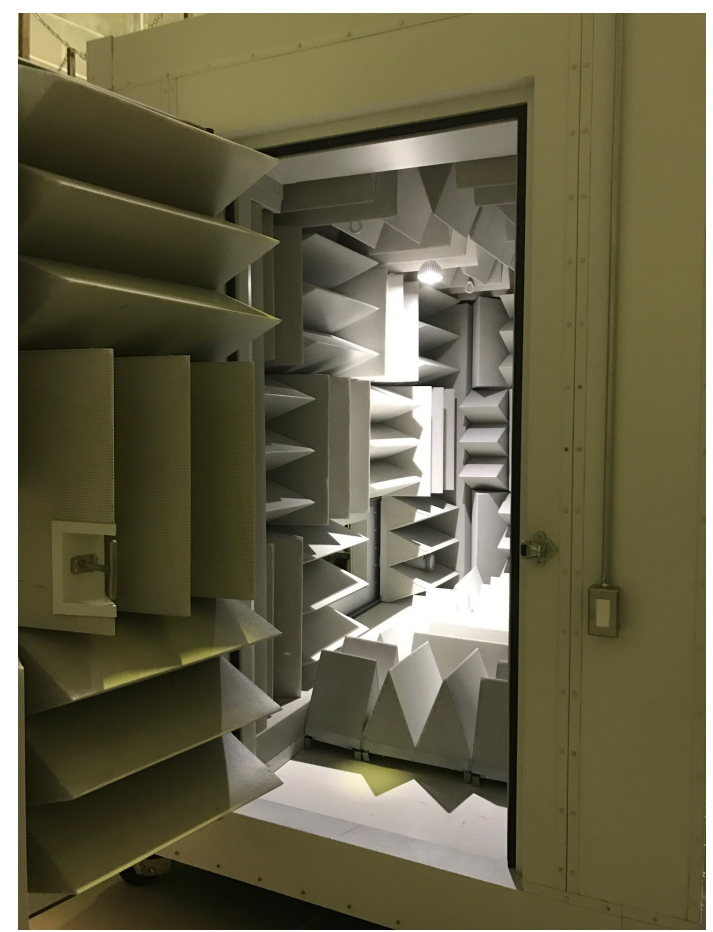

(a) Chamber with door

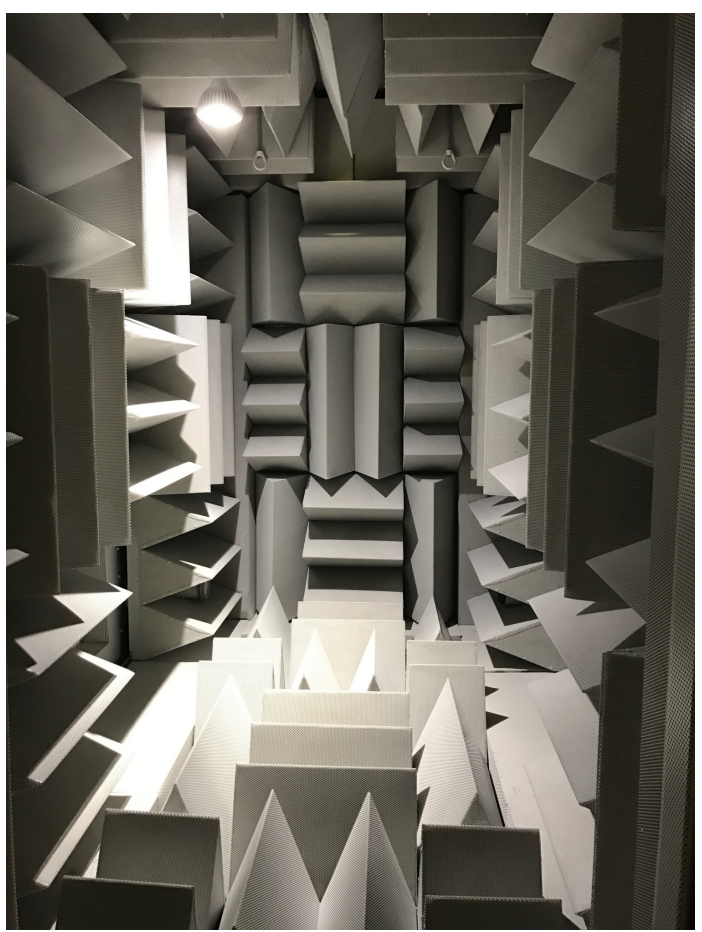

(b) Chamber with removable floor wedges

Figure 3.2: Completed anechoic chamber.

\subsection{Flexible High Pressure Air Hose}

The complete flexible high pressure air hose assembly described in Chapter 2 is shown below in Figure 3.3 , along with a photo of its interface connection with the upstream elbow pipe connected to the wind tunnel control valve. The hose assembly was purchased with carbon steel male National Pipe Thread (NPT) fittings. Low carbon steel ANSI B16.5 Class 150 standard $10.16 \mathrm{~cm}$ flanges with female threads were then placed onto these fittings for proper connection to the upstream and downstream wind tunnel components. 


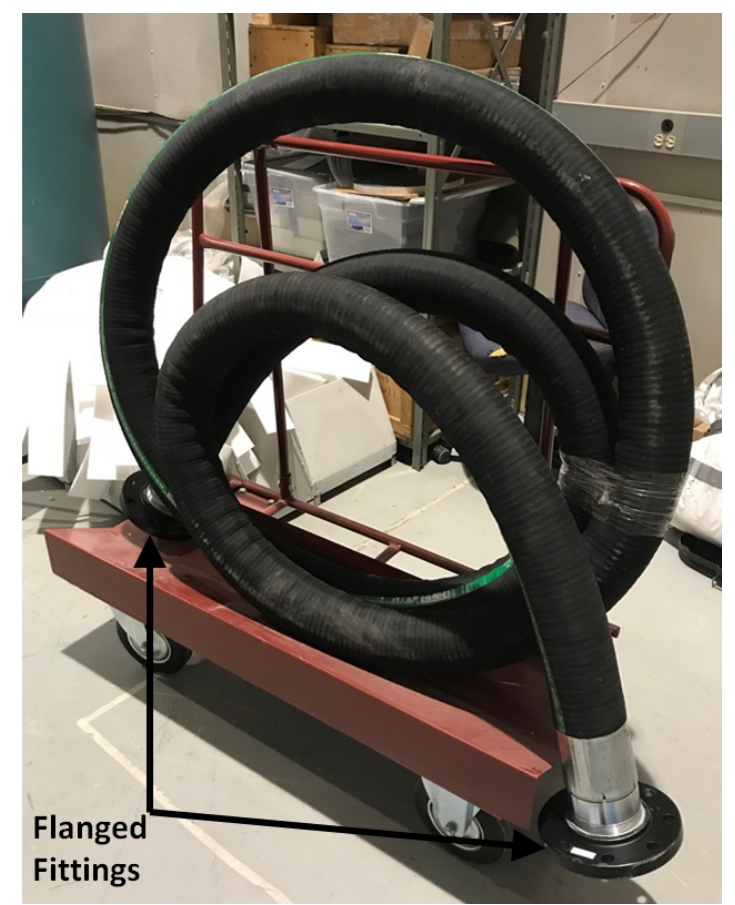

(a) Hose assembly

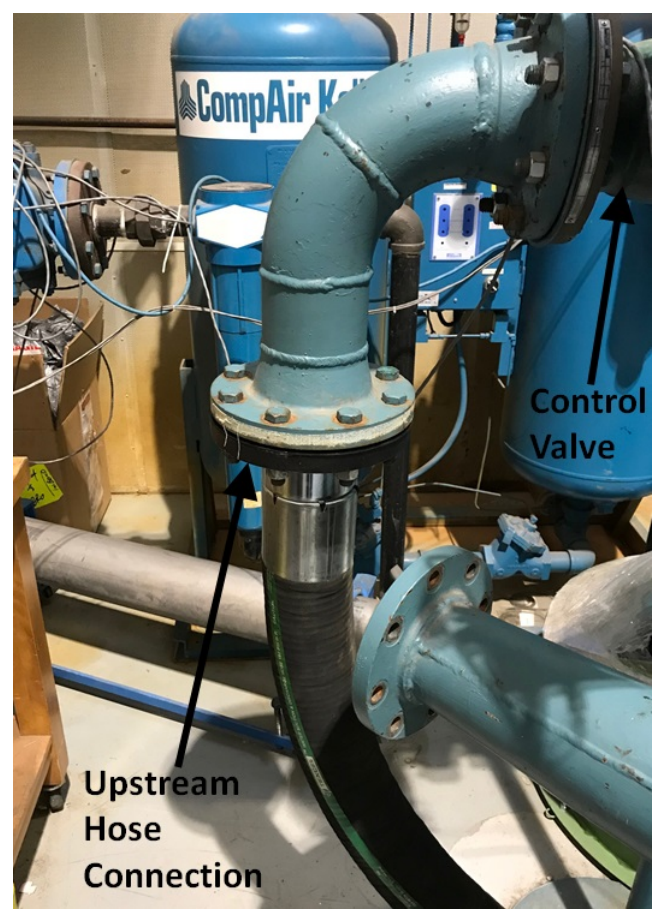

(b) Upstream connection

Figure 3.3: Flexible high pressure air hose assembly.

\subsection{Round-to-Rectangular Transition Duct}

The complete fabricated transition channel is contained in Figure 3.4 . The transition component was fabricated from several formed sheet metal and machined flange components which are shown in Figure 3.5 . The addition of the $5.08 \mathrm{~cm}$ long rectangular box like channel near the rectangular flange was implemented to house the upstream flow conditioning devices. The entire component assembly is welded together to form the final duct component. The formed sheet metal wall components are made of 11 gauge $(3.04 \mathrm{~mm})$ thick steel and the entire outer length of the assembly is $0.82 \mathrm{~m}$. The two flange components were machined to interface with the circular hose system and rectangular straight channel assembly as depicted in Figure 3.6. 


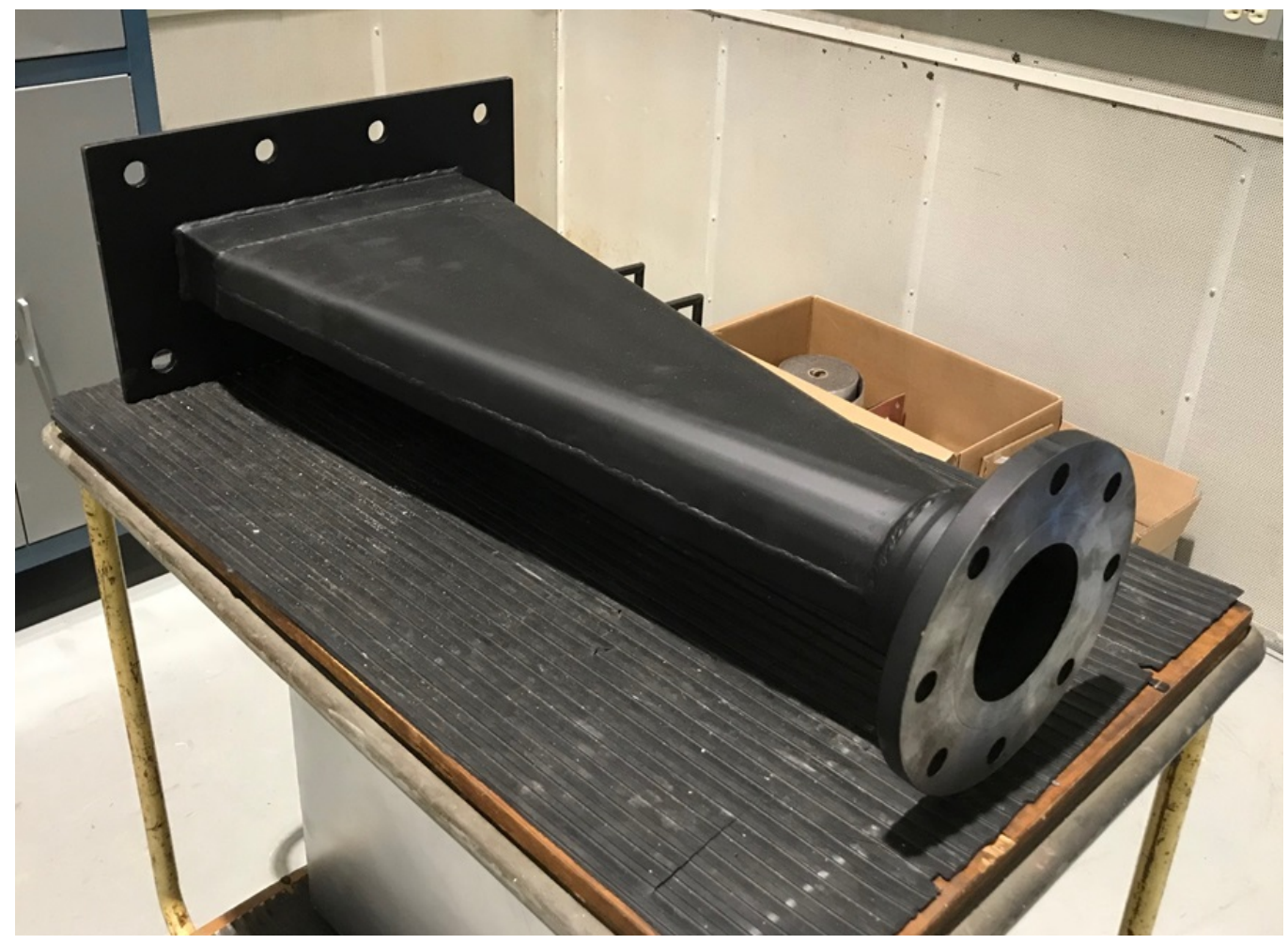

Figure 3.4: Fabricated transition channel.

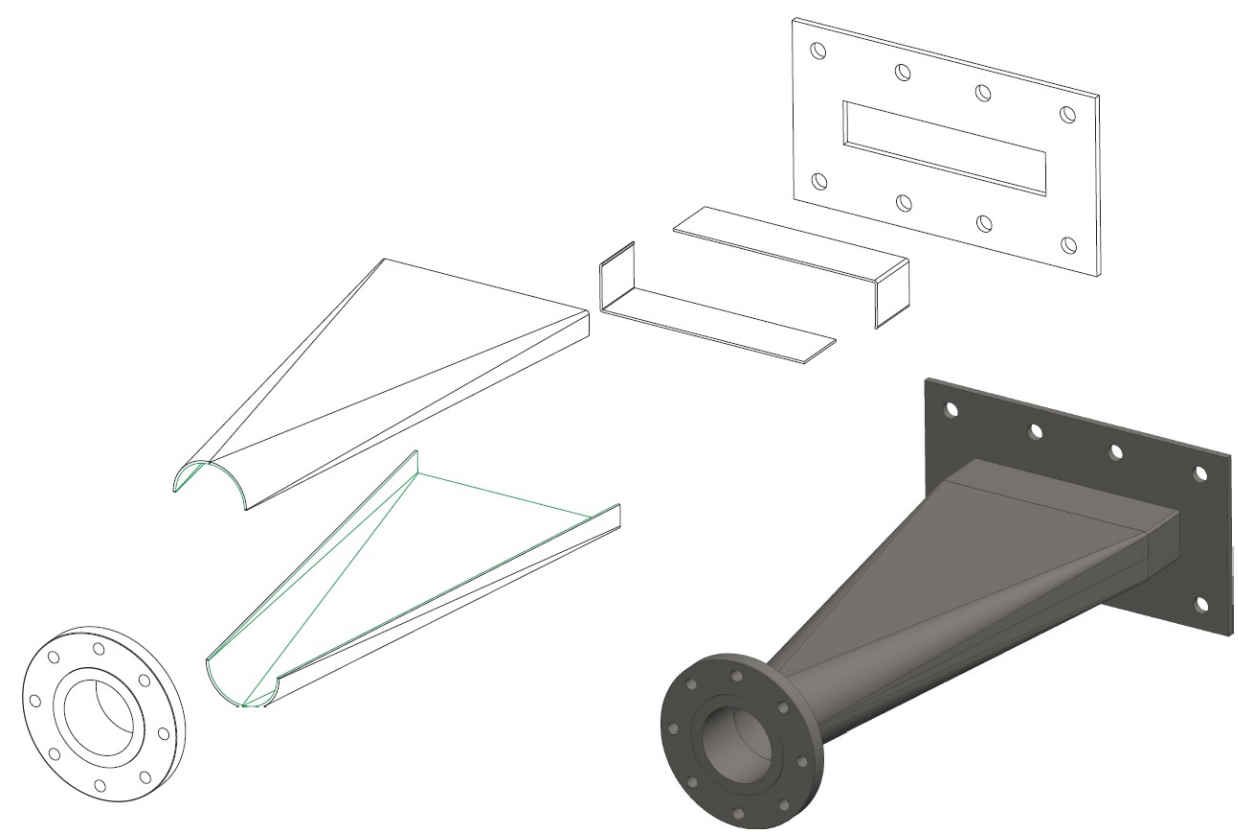

Figure 3.5: Transition channel welded component assembly. 


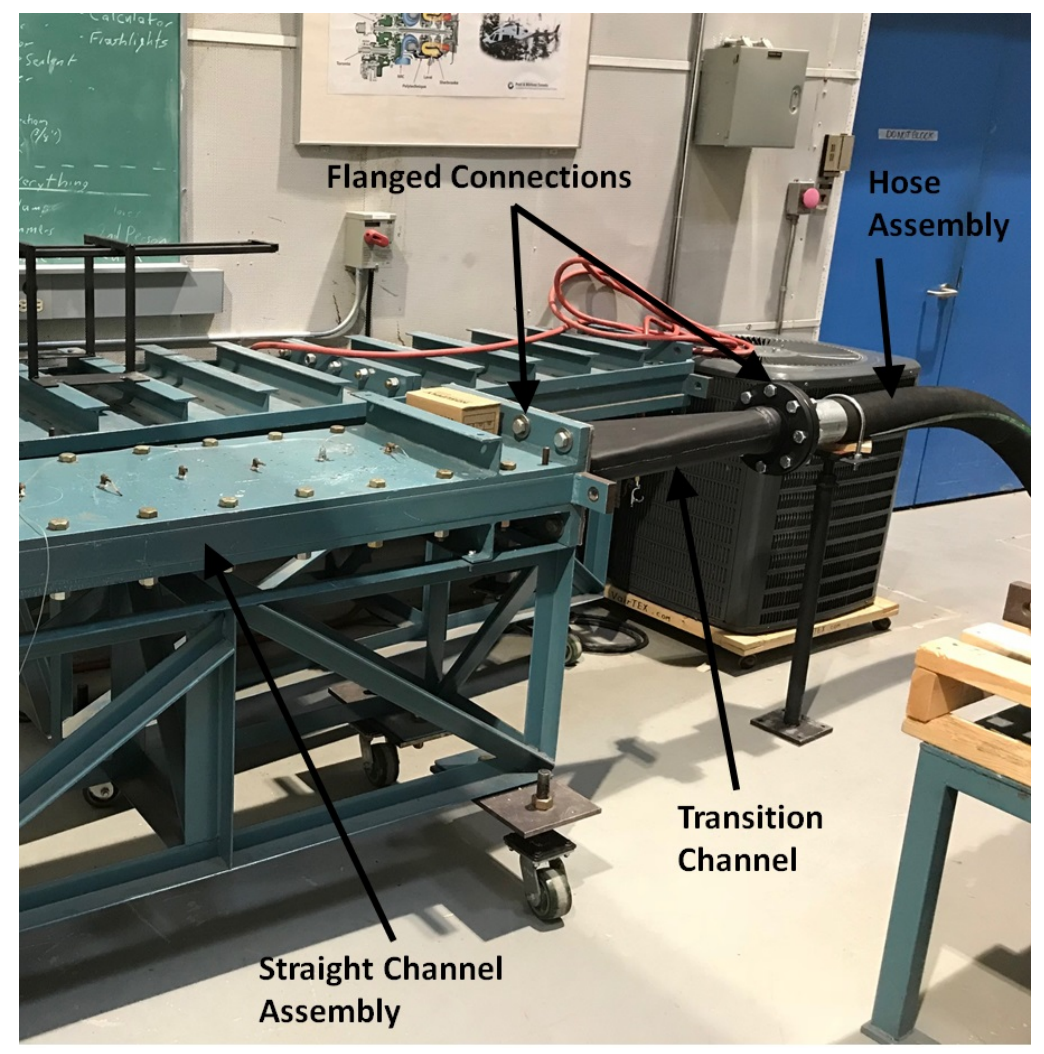

Figure 3.6: Transition channel connection interfaces.

\subsection{Flow Conditioning Devices}

The flow conditioning devices were fabricated from raw stock material. The honeycomb flow conditioner was cut to size from raw aluminum honeycomb cell material commonly used in structural applications. The flow conditioning screens were cut from raw stainless steel mesh screens. Figure 3.7 shows the placement of upstream flow conditioning devices (honeycomb and screen \#1) at the outlet of the transition duct. Figure 3.8 shows the placement of the second flow conditioning screen at the inlet of the nozzle assembly. Both components fit to the respective upstream and downstream openings in the straight channel assembly. 


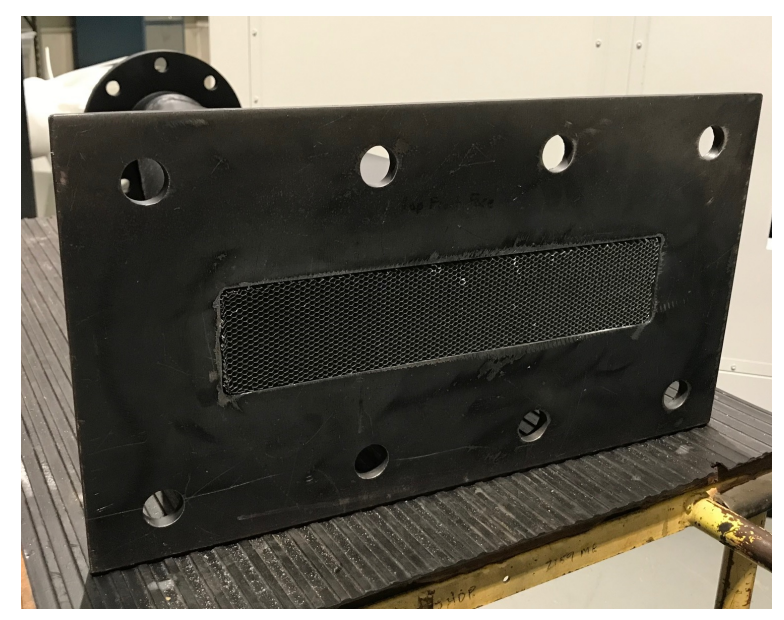

(a) Honeycomb cells

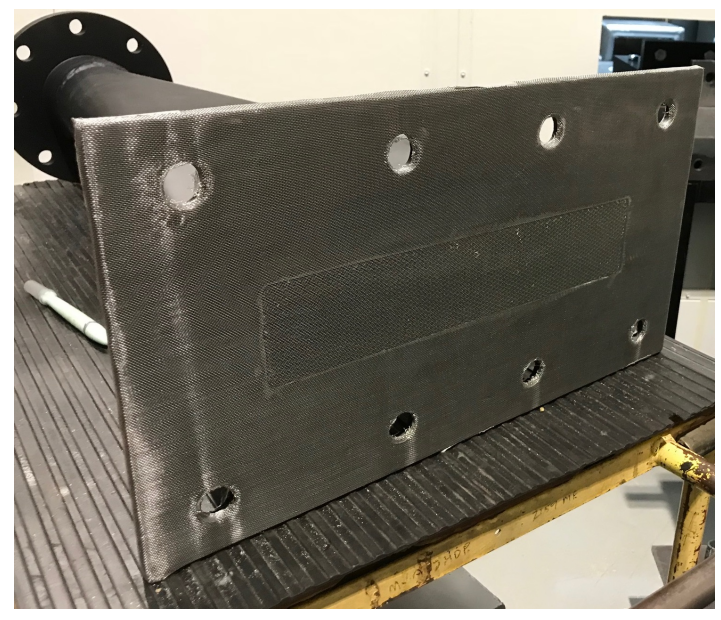

(b) Honeycomb with screen \#1

Figure 3.7: Upstream flow conditioning devices.

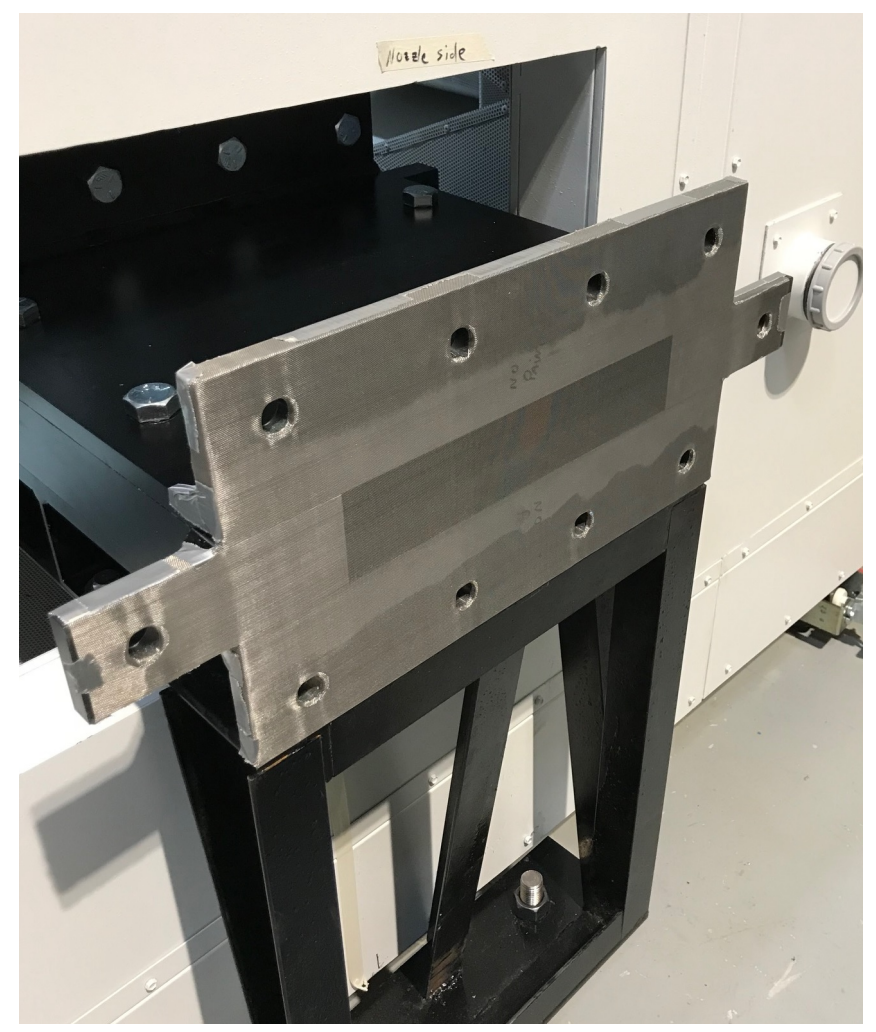

Figure 3.8: Downstream flow conditioning device (Screen \#2). 


\subsection{Nozzle Channel Assembly}

The nozzle channel assembly containing the interior duct profile outlined in Chapter 2 is displayed in Figure 3.9 along with a detailed view of its sub-components.

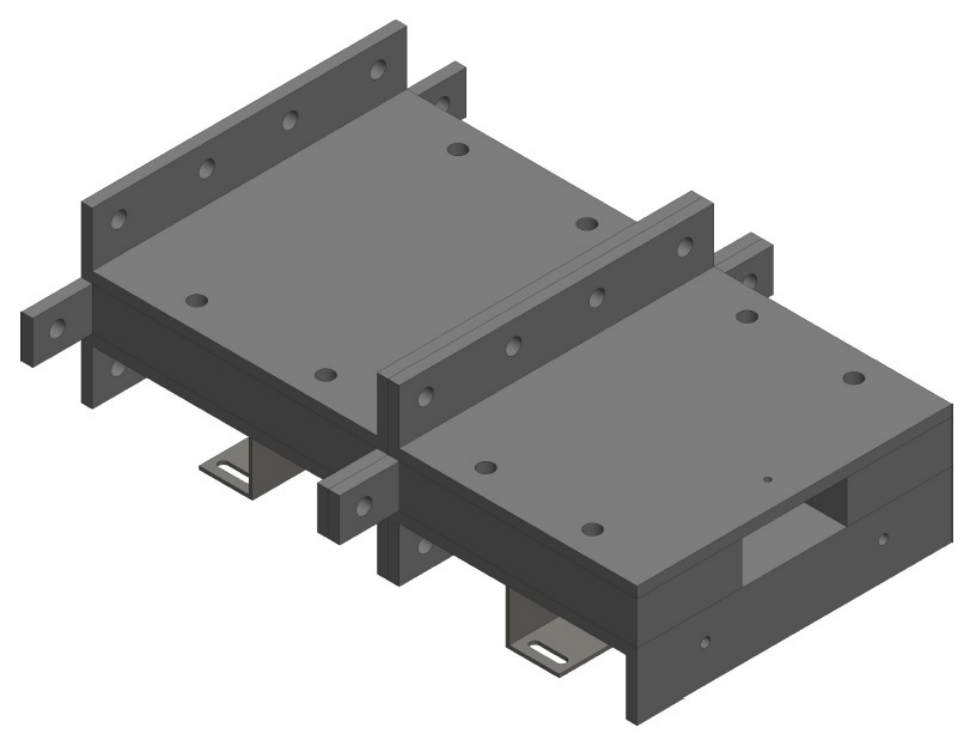

(a) Full assembly model

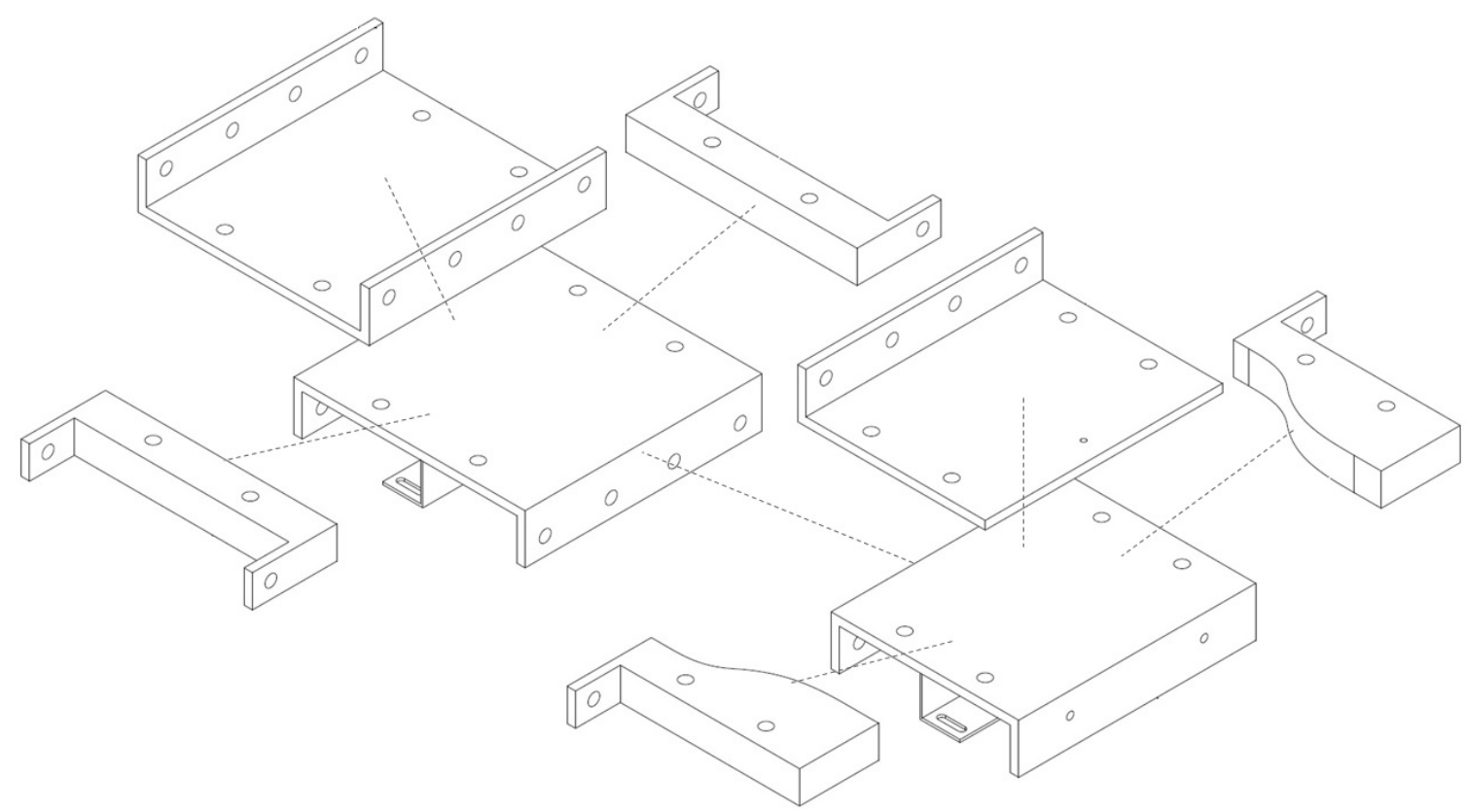

(b) Component explode view

Figure 3.9: Nozzle channel assembly. 
The design of the nozzle channel consists of machined steel side wall components (with the required wall/contraction profile) which are placed in between machined ceiling and floor components and fixed together with bolts. This design is similar to the construction of the straight channel assembly. Figure 3.10 contains photos of the fabricated assembly. The support structure was fabricated from welded steel plates and angle bars.

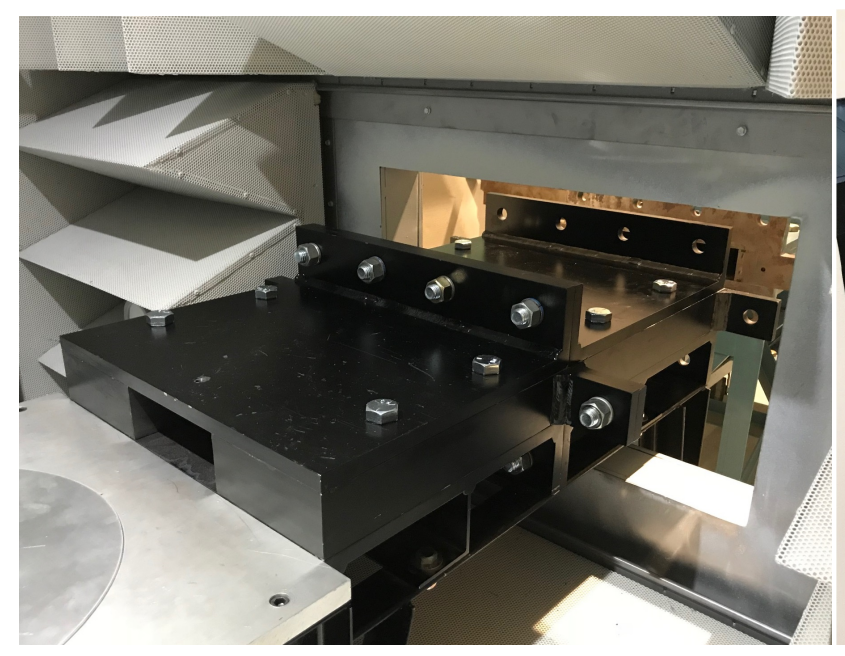

(a) Chamber interior view

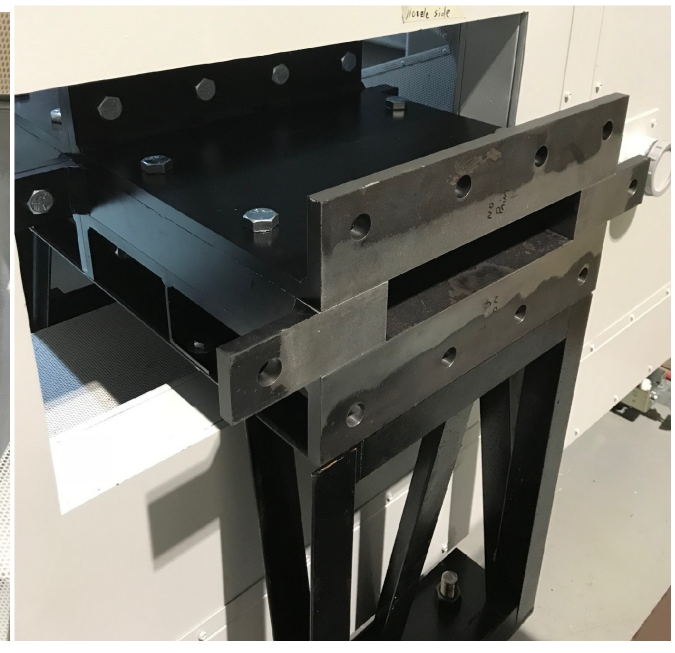

(b) Chamber exterior view

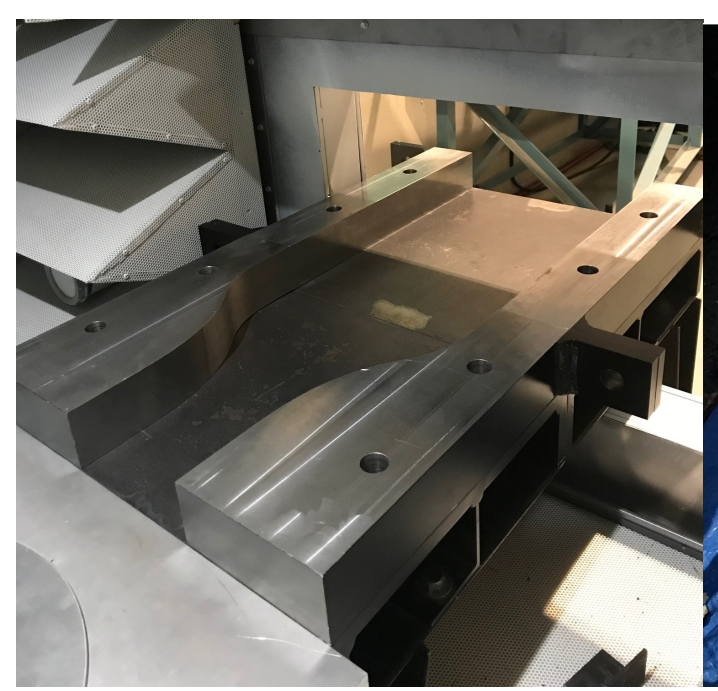

(c) Channel interior view

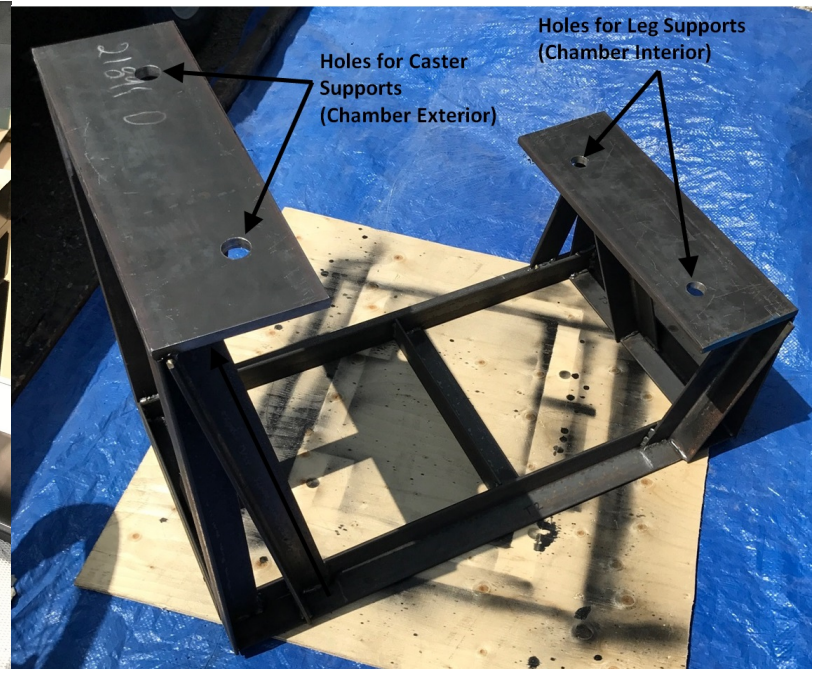

(d) Nozzle support structure (upside down)

Figure 3.10: Fabricated nozzle system. 
The entire nozzle assembly is attached on the support structure which is fed through and fixed to the floor inside the chamber. The structure was fitted with two 1000 lbs casters on the exterior side of the chamber so the entire system can move. The open space around the nozzle and structure in the feed-through hole was covered with $10.16 \mathrm{~cm}$ of acoustic fiberglass insulation and covered with pieces of plywood. All other gaps were sealed using acoustic grade adhesive chalk. Figure 3.11 shows this cover where the interior surface was treated with acoustic foam wedge sheets.

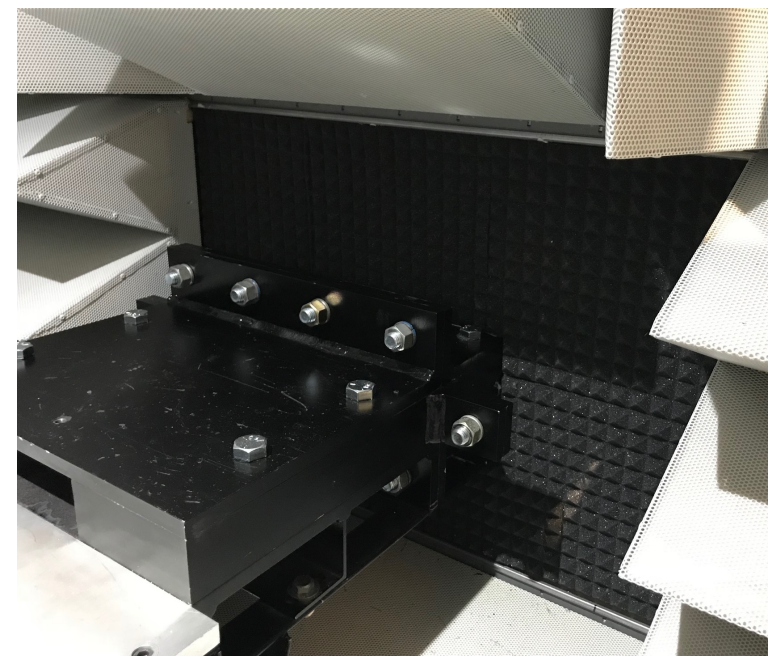

(a) Interior side

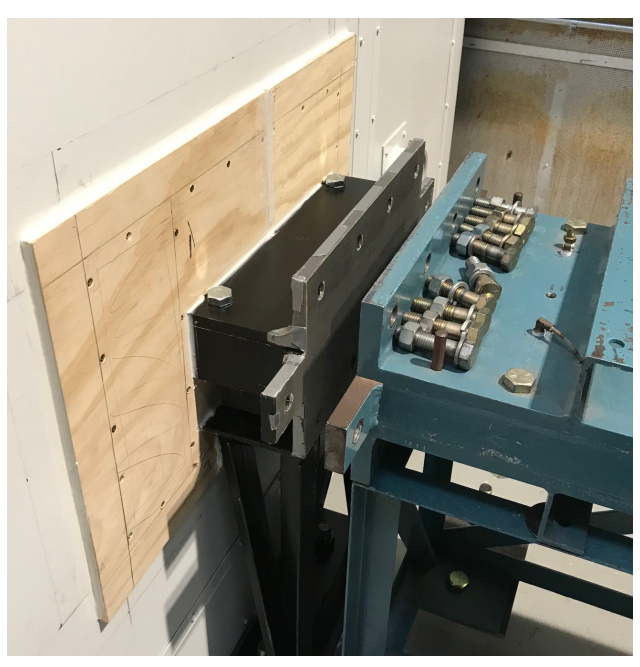

(b) Exterior side

Figure 3.11: Nozzle feed-through hole cover.

\subsection{Chamber Flow Exhaust System}

The chamber flow exhaust system components (excluding all support structures) were fabricated by VibroAcoustics and HTS. The complete exhaust system 3-D model is displayed in Figure 3.12. Figure 3.13 contains a more detailed look at the major components of the exhaust system with applicable labels and dimensions for clarity. 


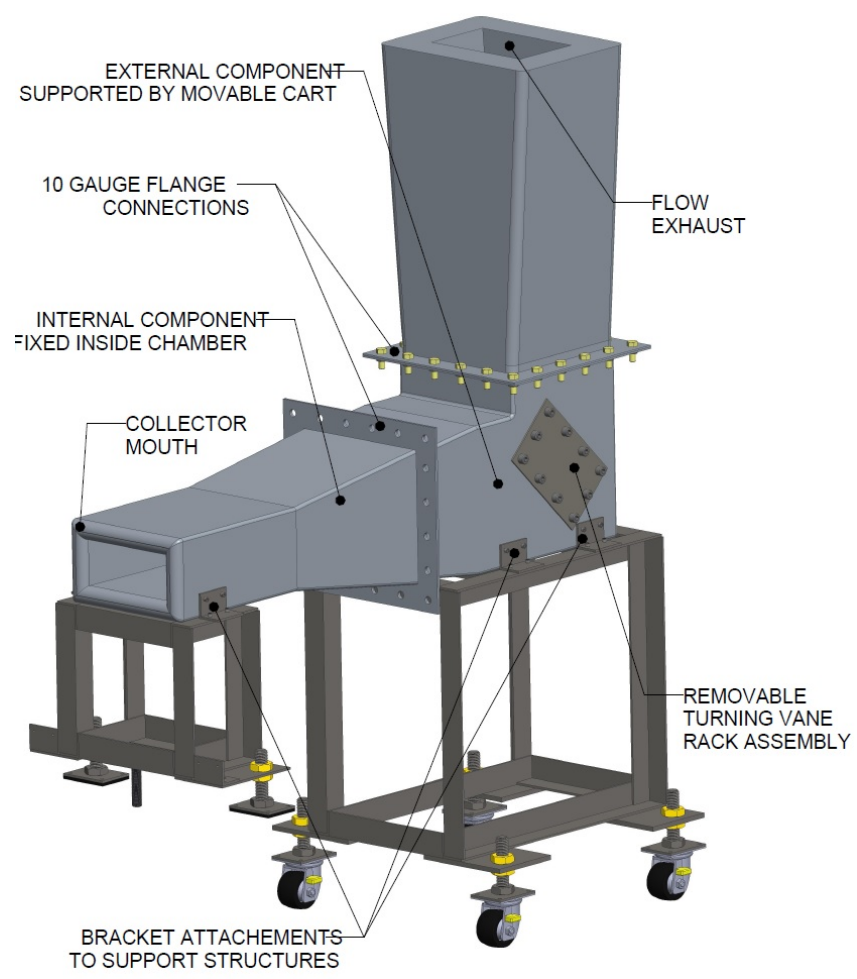

Figure 3.12: Complete chamber flow exhaust system model.

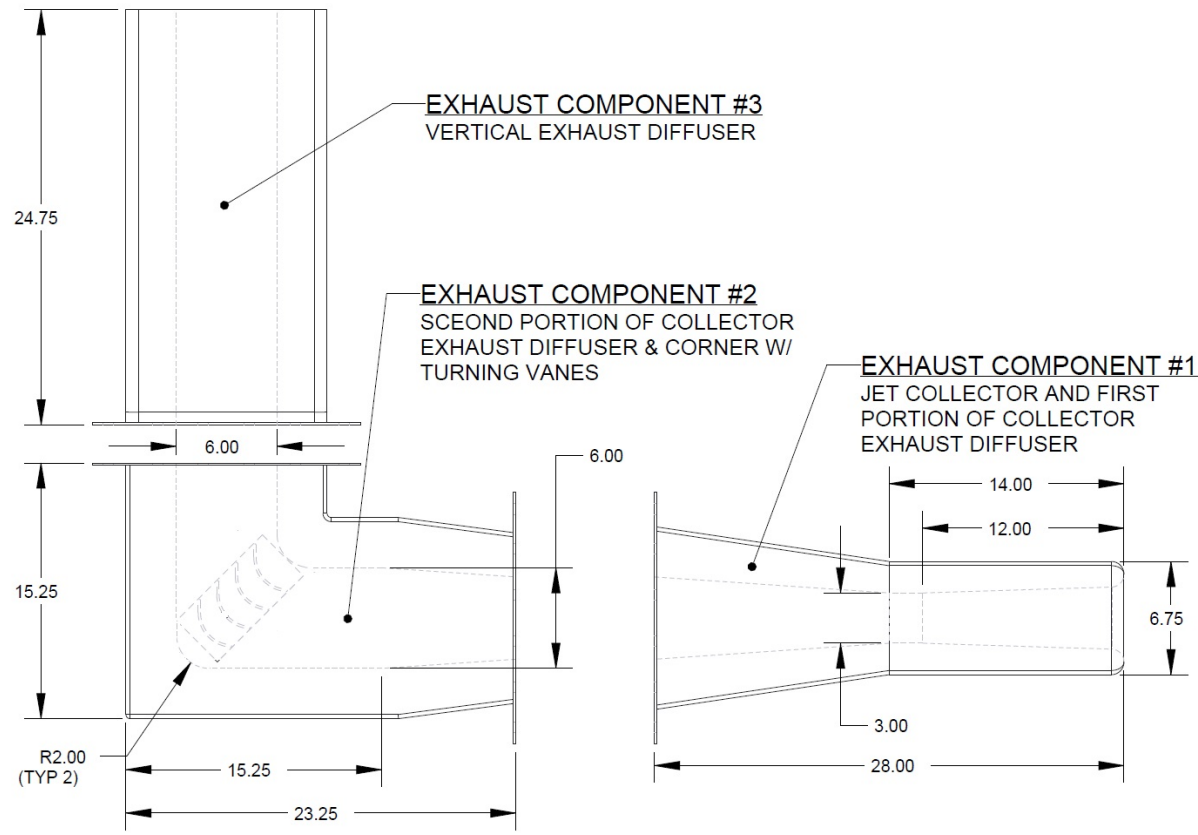

Figure 3.13: Exhaust system major component drawings (all dim in inches). 
The maximum duct wall thickness of the system is $7.62 \mathrm{~cm}$. The general construction of the duct walls consist of the following material starting from the inside: galvanized perforated sheet metal (12 gauge, $23 \% O A)$; stainless steel mesh (18x18 cells/in); fiberglass cloth; $6.35 \mathrm{~cm}$ thick steel wool; fiberglass bulk insulation; 4.76 $\mathrm{cm}$ steel outer casing. This type of duct design is similar to a silencer which helps to suppress background noise propagation upstream and into the chamber. The first major exhaust component however was designed with solid internal walls to reduce system pressure losses due to the higher flow speeds in that portion of the duct work.

The first major component of the exhaust system contains the jet collector and a portion of the collector exhaust diffuser. This component is designed to remain fixed inside the chamber to an internal support. The component is feed-through the second hole on the exhaust side of the chamber with the flange of the component protruding just slightly outside of the chamber. The spacing around the component in the feed-through hole is sealed in a similar manner to the nozzle system. Figure 3.14 contains several photos showing the final fabricated component. The support was fabricated at Carleton in a similar manner as the nozzle support structure.

The second major exhaust system component contains the remaining portion of the collector exhaust diffuser as well as the $90^{\circ}$ corner with turning vanes. The third component contains the vertical exhaust diffuser. Both components are designed to remain fixed on a movable support cart with casters. The entire system can then be attached to the internal exhaust component via the flange connection when the tunnel is in use. Figure 3.15 shows both fabricated components, along with its connection with the chamber. A detailed look at the fabricated turning vane rack assembly can be seen in Figure 3.16 . 


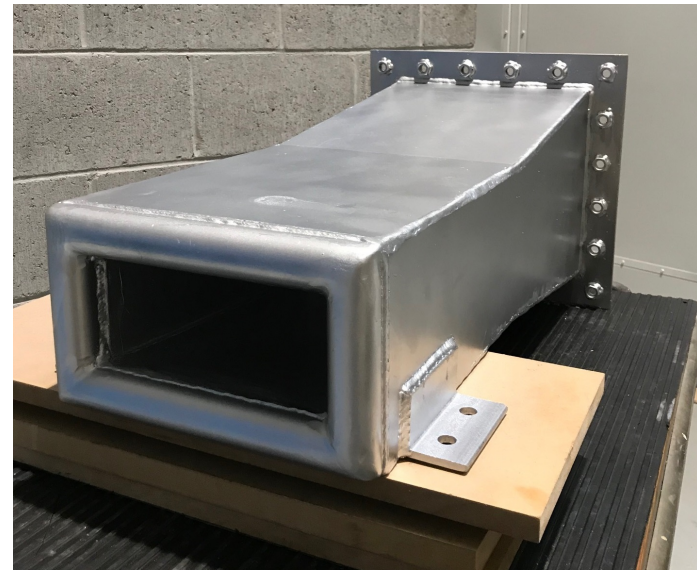

(a) Front view

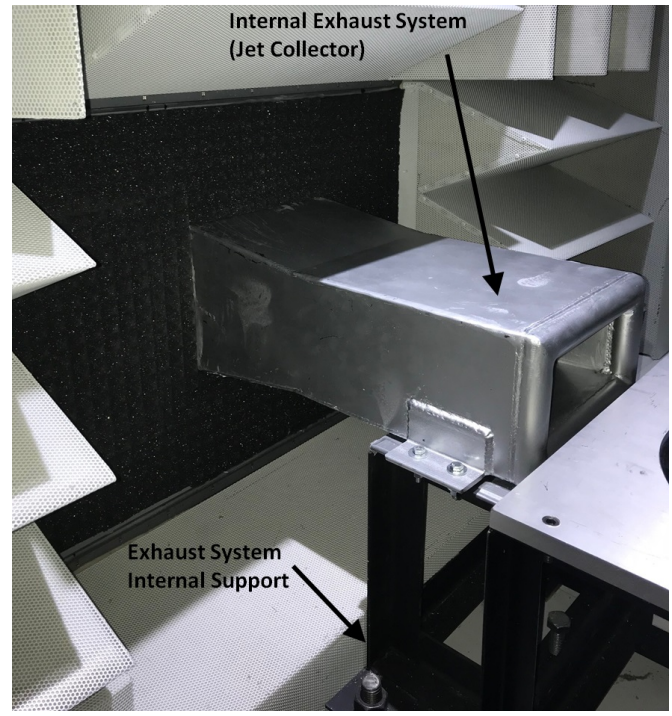

(c) Internal installation

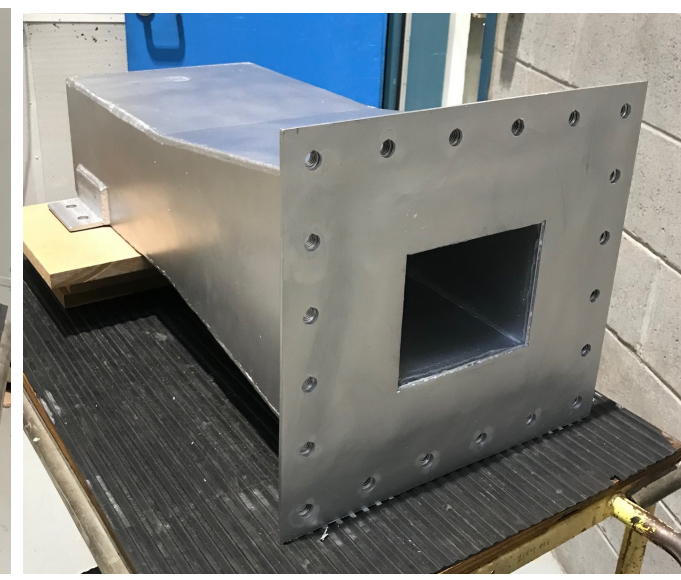

(b) Rear view

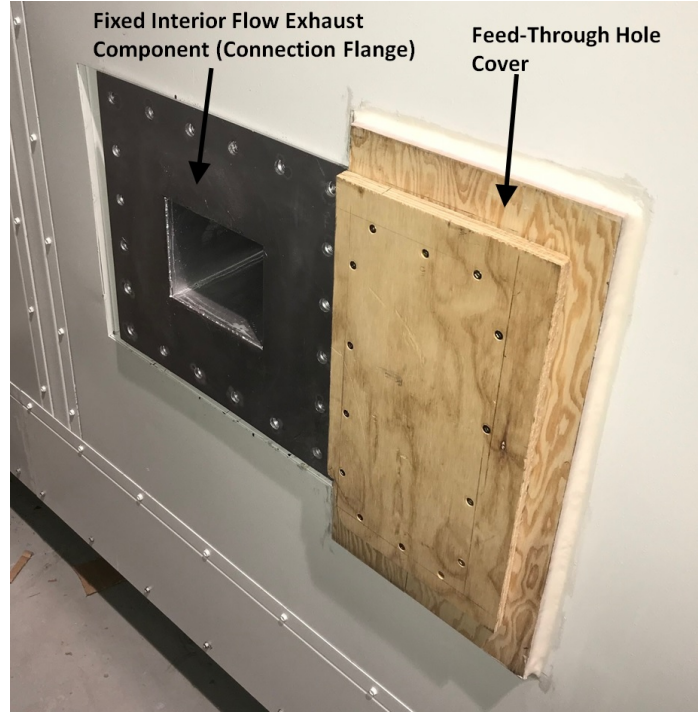

(d) External installation

Figure 3.14: Chamber internal flow exhaust component. 


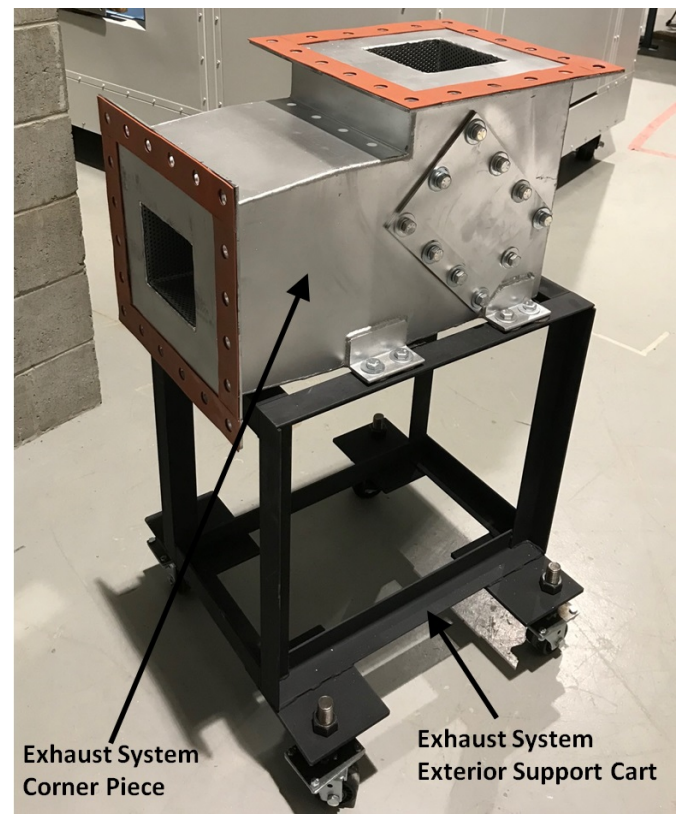

(a) Exhaust corner

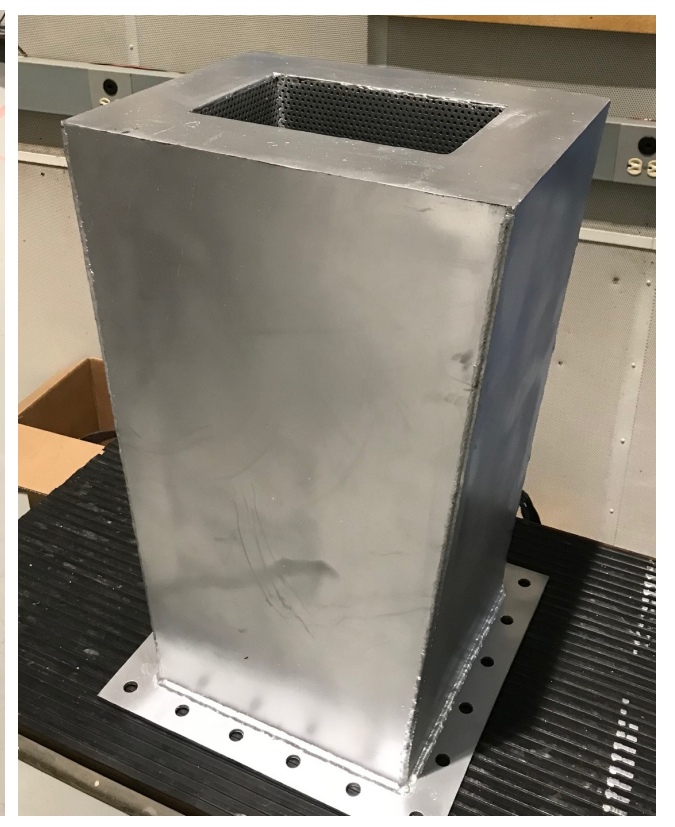

(b) Vertical exhaust diffuser

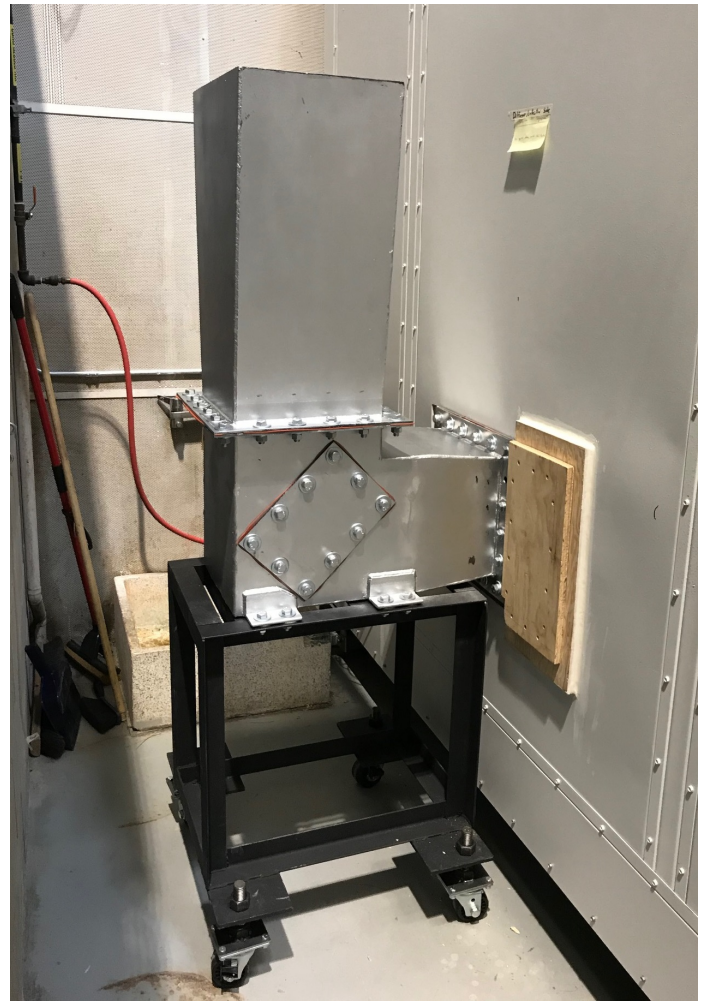

(c) Assembly connection interface

Figure 3.15: Chamber external flow exhaust assembly. 


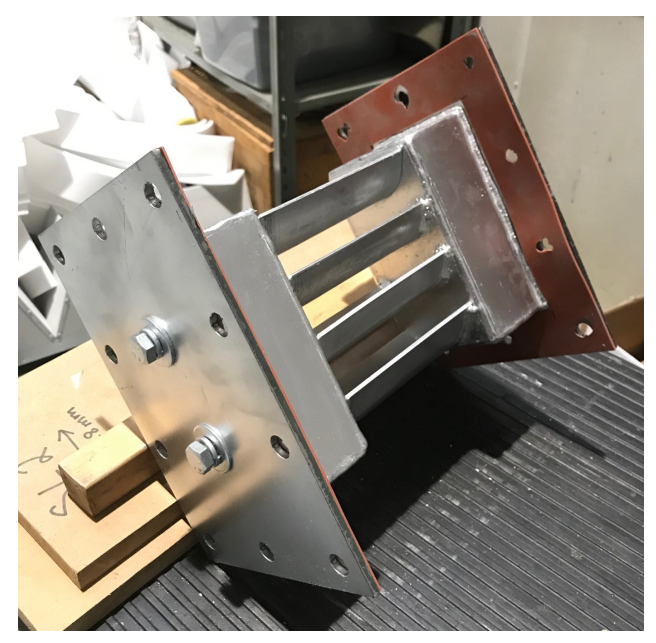

(a) Fabricated assembly

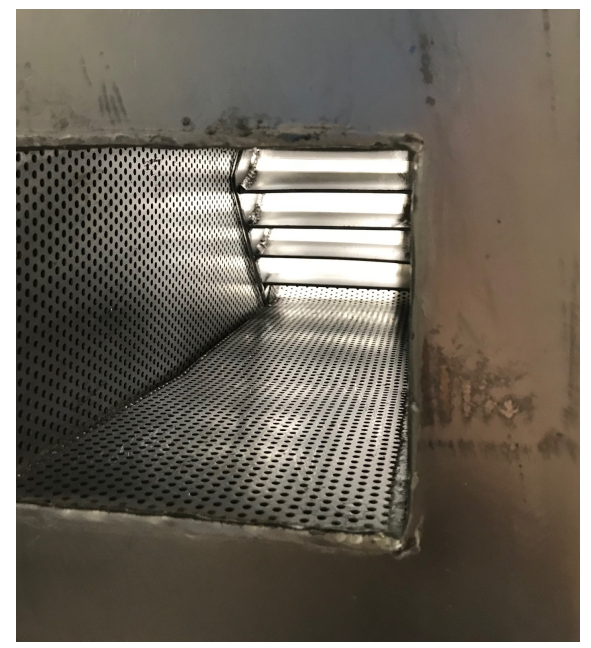

(c) Installation view \# 2

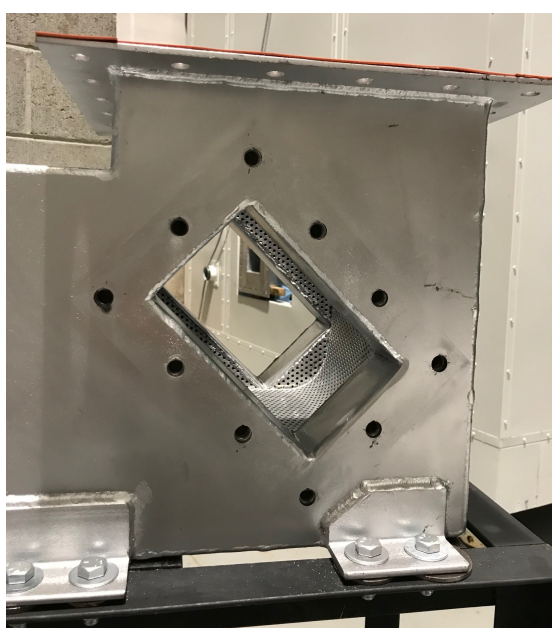

(b) Installation slot

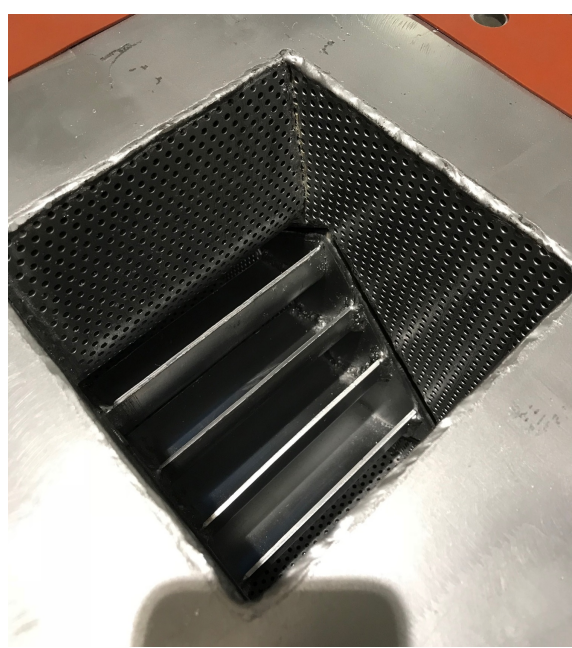

(d) Installation view \# 2

Figure 3.16: Turning vane rack assembly.

\subsection{Chamber Test Section}

The fabricated flush mounted TBL test panel system is shown in Figure 3.17. The panel is fixed on top of a welded support structure that is adjustable in height. The panel support plate was machined from a $19.05 \mathrm{~mm}$ thick aluminum plate with 50.8 x $50.8 \mathrm{~cm}$ planform dimensions. A centre hole was water-jet cut to an interference fit to house the flush mounted acrylic test panel. Please refer to [17] for complete 
design details regarding the acrylic panel. The entire panel set-up is designed to fit in the test section, flush mounted with the floor of the nozzle channel. The panel support structure is also fixed to the nozzle and collector support structures. Figure 3.18 contains photos of the completed test section.

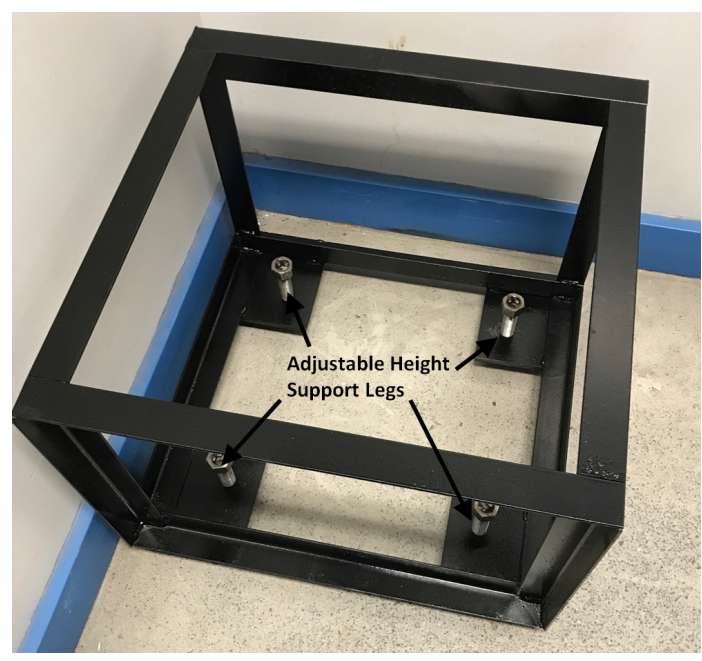

(a) Support structure

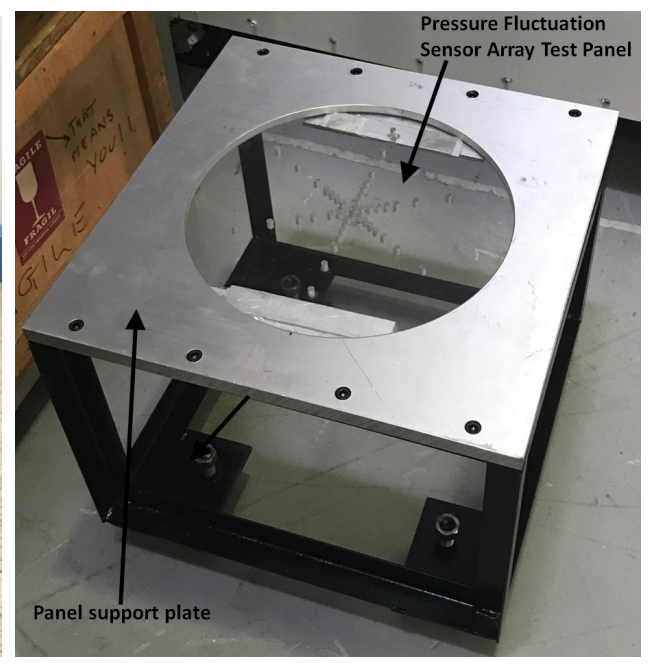

(b) Complete assembly

Figure 3.17: Test section TBL test panel system.

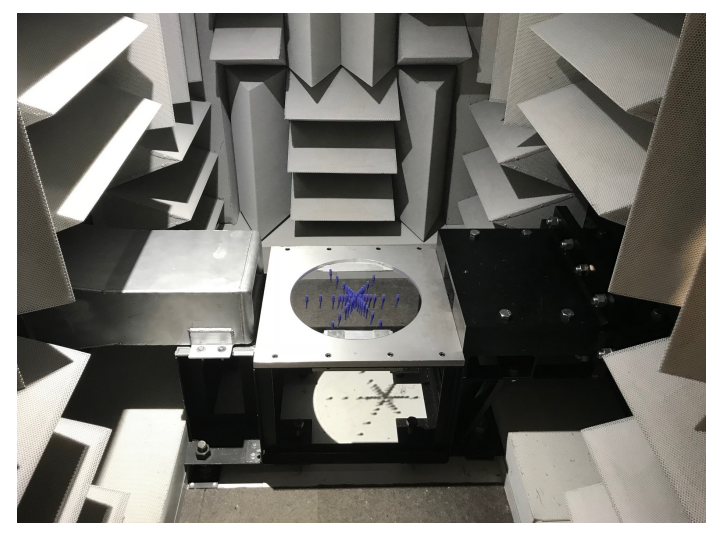

(a) View \#1

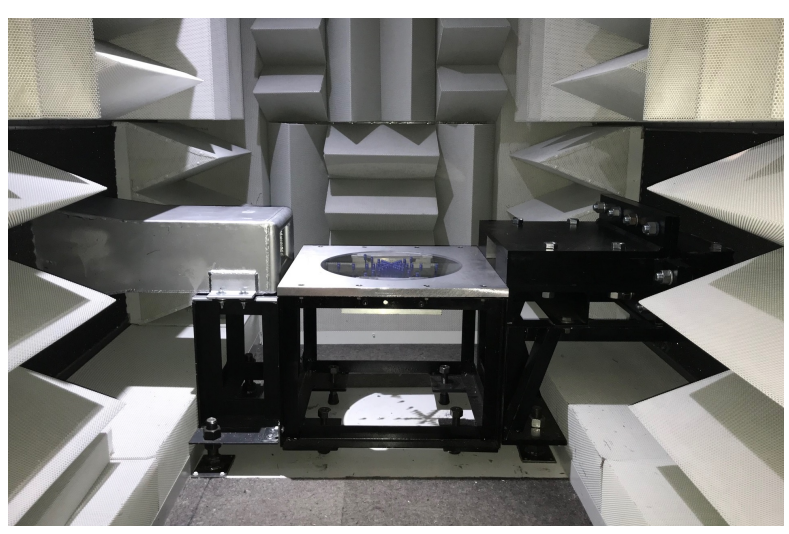

(b) View \#2

Figure 3.18: Completed chamber test section. 


\subsection{Acoustic Treatments}

Various acoustic treatments were preformed to the wind tunnel components housed within the chamber. These include surface treatments to exposed solid surfaces to improve with sound reverberation and absorption. Silencers were also fitted over the ventilation holes on the ceiling of the chamber. The silencers were fabricated in-house using a 12 gauge steel outer casing and 16 gauge internal perforated sheet metal walls with a $41 \% O A$ and a Ti of roughly 2300 (above the minimum of 2000 as described in Chapter 2). Figure 3.19 contains photos of these treatments and Figure 3.20 shows the final testing configuration of the chamber with the acoustic floor wedges.

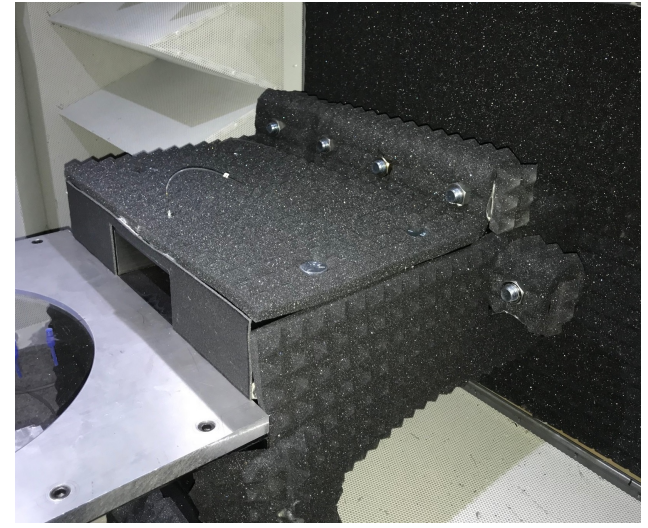

(a) Nozzle

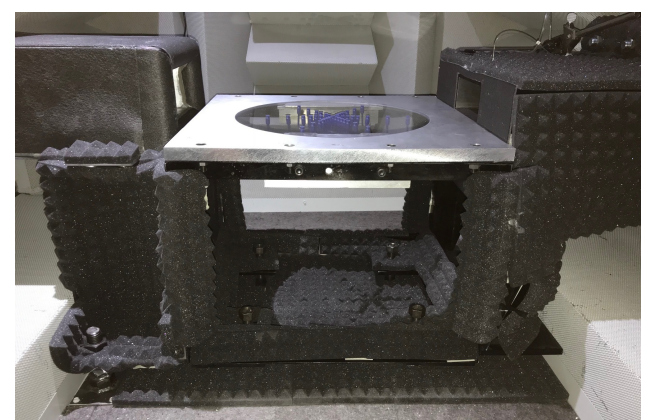

(c) Test section support structures

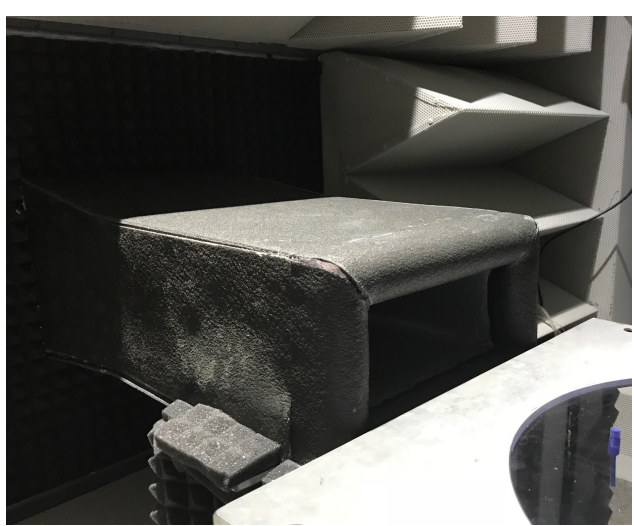

(b) Collector

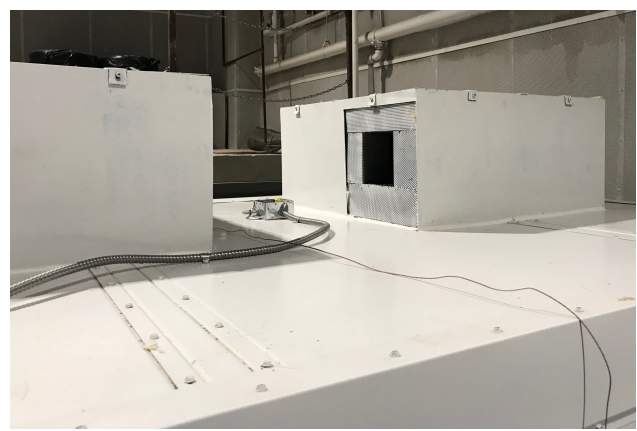

(d) Ventilation silencers

Figure 3.19: Component acoustic treatments. 


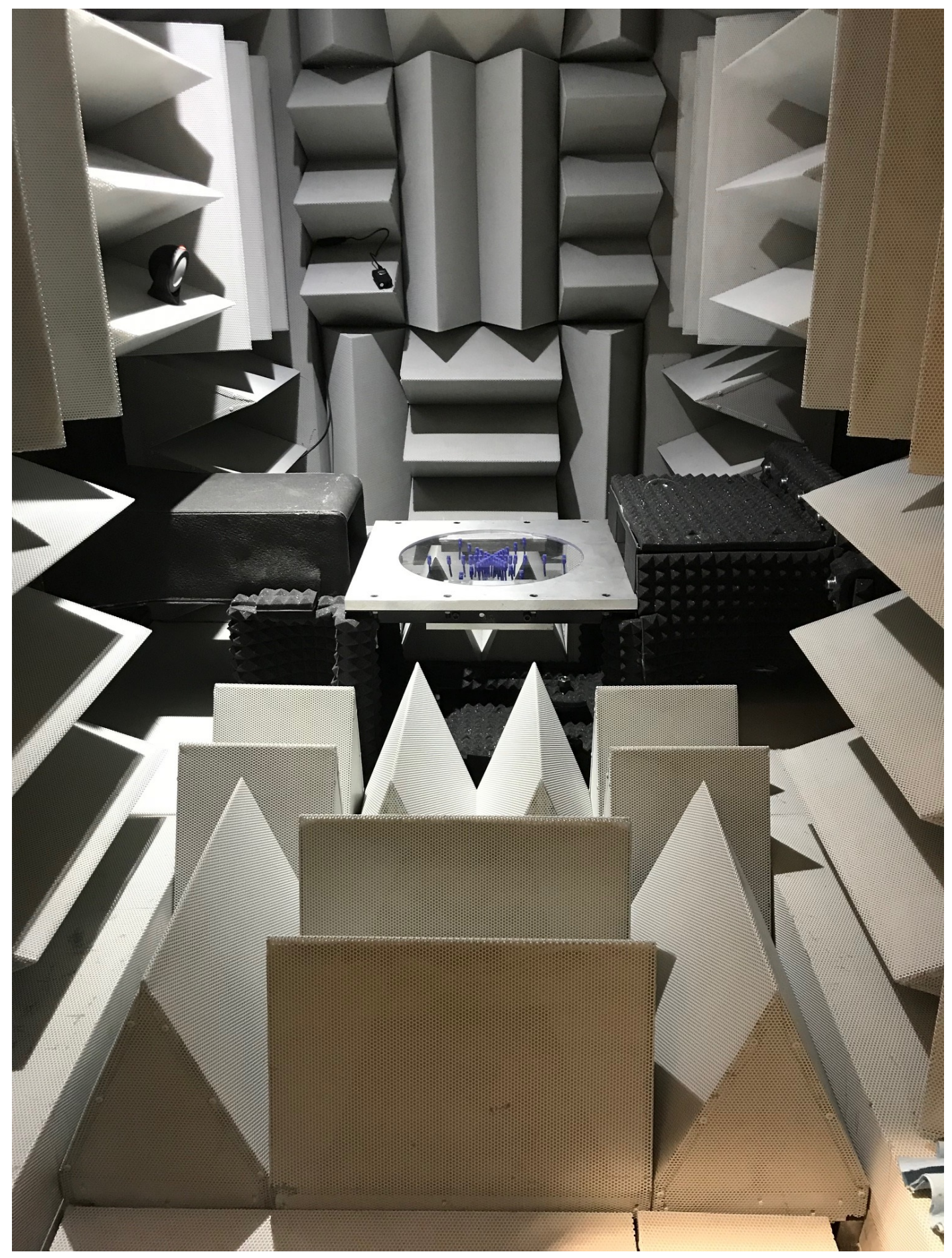

Figure 3.20: Complete anechoic test section. 


\section{Chapter 4}

\section{Experimental Methods and Facility Operation}

This chapter details the experimental methods and procedures for operation of the HSAWT facility. First, the methodology of operating a blowdown wind tunnel is described along with the required measurement systems. The existing control system and data acquisition hardware is then discussed. Finally, the newly implemented instrumentation and measurement system hardware is presented; as well as how it interfaces with the existing facility equipment.

\subsection{Blowdown Wind Tunnel Operation}

A typical blowdown wind tunnel with a subsonic test section has the following highlevel component schematic displayed in Figure 4.1. When the tunnel safety shut-off valve is fully open to engage in operation, flow downstream of the control valve throughout the wind tunnel is kept steady in terms of test section Mach number by incrementally opening the control valve to allow more area mass flow as the storage tanks are decreasing in pressure. Once the valve is fully opened in a run, it can no 


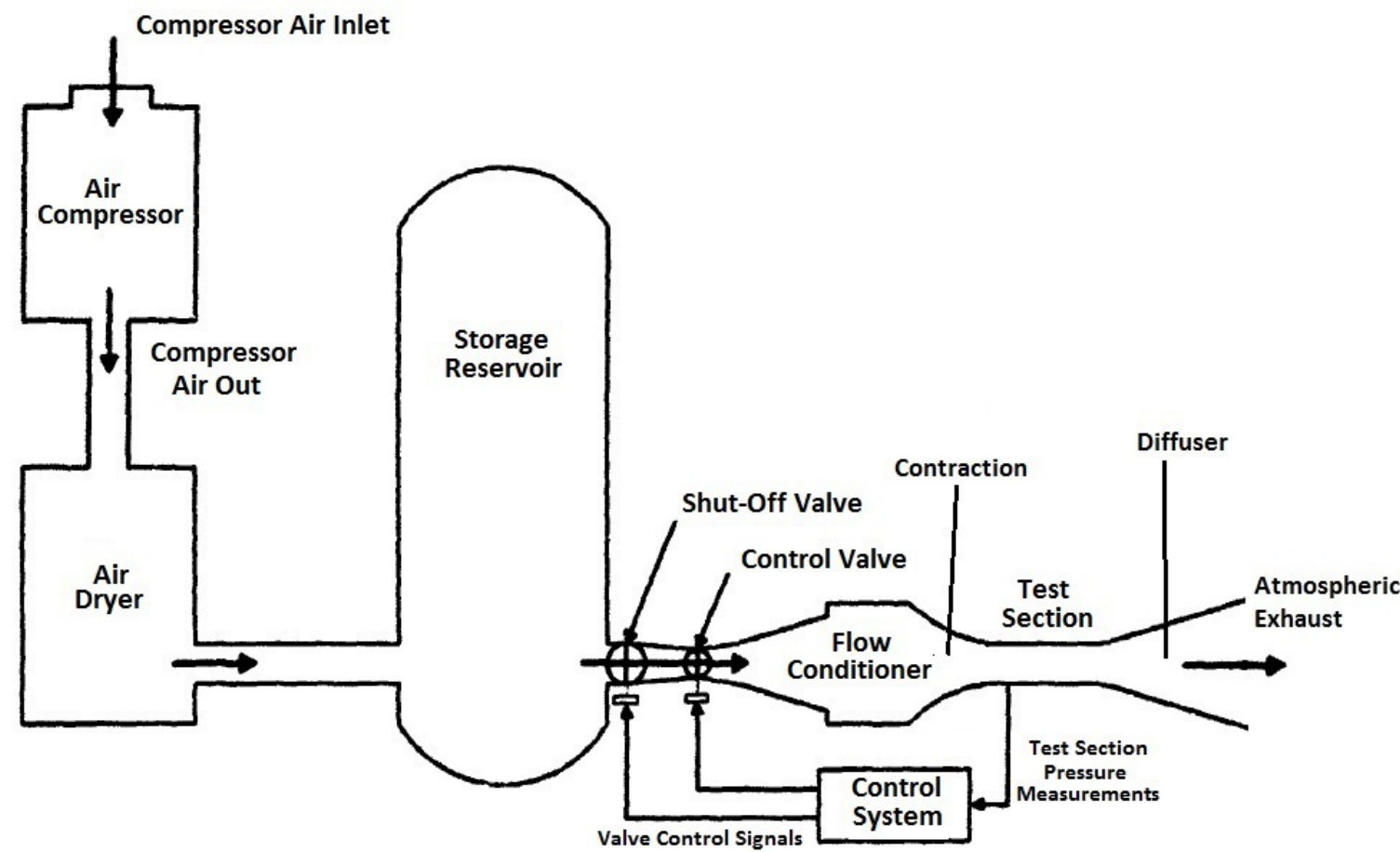

Figure 4.1: Schematic of a typical idealized subsonic blowdown wind tunnel (adapted from [47]).

longer open further to compensate for the loss in tank pressure, thus signaling the end of a controlled run. This phenomena is described using the compressible mass flow rate relationship displayed in Equation 4.1] [76]:

$$
\dot{m}=\frac{A P_{o} \sqrt{\gamma}}{\sqrt{T_{o} R}}\left[M\left(1+\frac{\gamma-1}{2} M^{2}\right)^{-\frac{\gamma+1}{2(\gamma-1)}}\right]
$$

where a constant governed mass flow rate, $\dot{m}$, and Mach number, $M$, requires that the duct area, $A$, be continuously enlarged to compensate for the decrease in total or stagnation pressure, $P_{o}$, for a given total temperature, $T_{o}$. The ideal gas specific heat ratio, $\gamma$, and specific gas constant, $R$, correspond to values of 1.4 and $287 \mathrm{JKg}^{-1} \mathrm{~K}^{-1}$ respectively for air.

Control of the valve is facilitated by a Proportional-Integral-Derivative (PID) feedback control system to keep the test section Mach number constant throughout a run. 
The control system utilizes the error between the desired and measured test section Mach number to provide a relative control response in the form of a supply voltage output to the valve positioner. The test section Mach number can be computed from absolute measurements of total and static pressure at the desired control point. The relationship between Mach number, $M$, total pressure, $P_{o}$, and static pressure, $P$, is given below in Equation 4.2 for compressible flow [76].

$$
\frac{P_{o}}{P}=\left(1+\frac{\gamma-1}{2} M^{2}\right)^{\frac{\gamma}{\gamma-1}}
$$

\subsubsection{Measurement System Requirements and Locations}

Control of the wind tunnel requires mandatory measurements of the absolute static and total pressure at the required control point just before the nozzle exit. The usual measurement technique of total and static pressure involves placing a pitot-static probe in the freestream, which would disturb the flow entering the test section if placed right at the nozzle orifice. An alternative method is to separate the measurements by placing a total pressure probe at some position upstream of the test section and to measure the static pressure using a tap drilled on the top surface of the nozzle section where the Mach number is to be controlled. Both measurements have to be corrected for the true pressure values at the control point. The difference between a total pressure measurement at the nozzle exit and some point upstream is equal to the pressure losses that occur across both points. The difference between the static pressure measurements will only be due to the error produced by the tap geometry if the tap is placed at the control point (usually a positive pressure error) [83].

In addition to the pressure measurements for control of the wind tunnel, measurements of the tank stagnation pressure and the total temperature of the flow are required. Monitoring the tank pressure is important to ensure that a run is conducted 
when the storage tanks are at full capacity and the compressor is in by-pass. A flow total temperature measurement is useful for calculating the mass flow rate in a given run. It is also crucial to examine the drop in flow temperature that occurs naturally due to the expansion of the air out of the storage tanks and to assess its impact on the test section Reynolds number (a common drawback of a blowdown wind tunnel configuration). If it is assumed that heat transfer effects with the flow and the surrounding duct walls is negligible (adiabatic wall assumption), the total temperature at the control point can be measured directly with a thermocouple placed within the flow at any upstream point with no correction factors needed (this assumption was verified by early studies conducted by Jeffries [47] for the original tunnel configuration). Figure 4.2 contains a schematic of the wind tunnel with the selected location of the measurement systems outlined with red arrows.

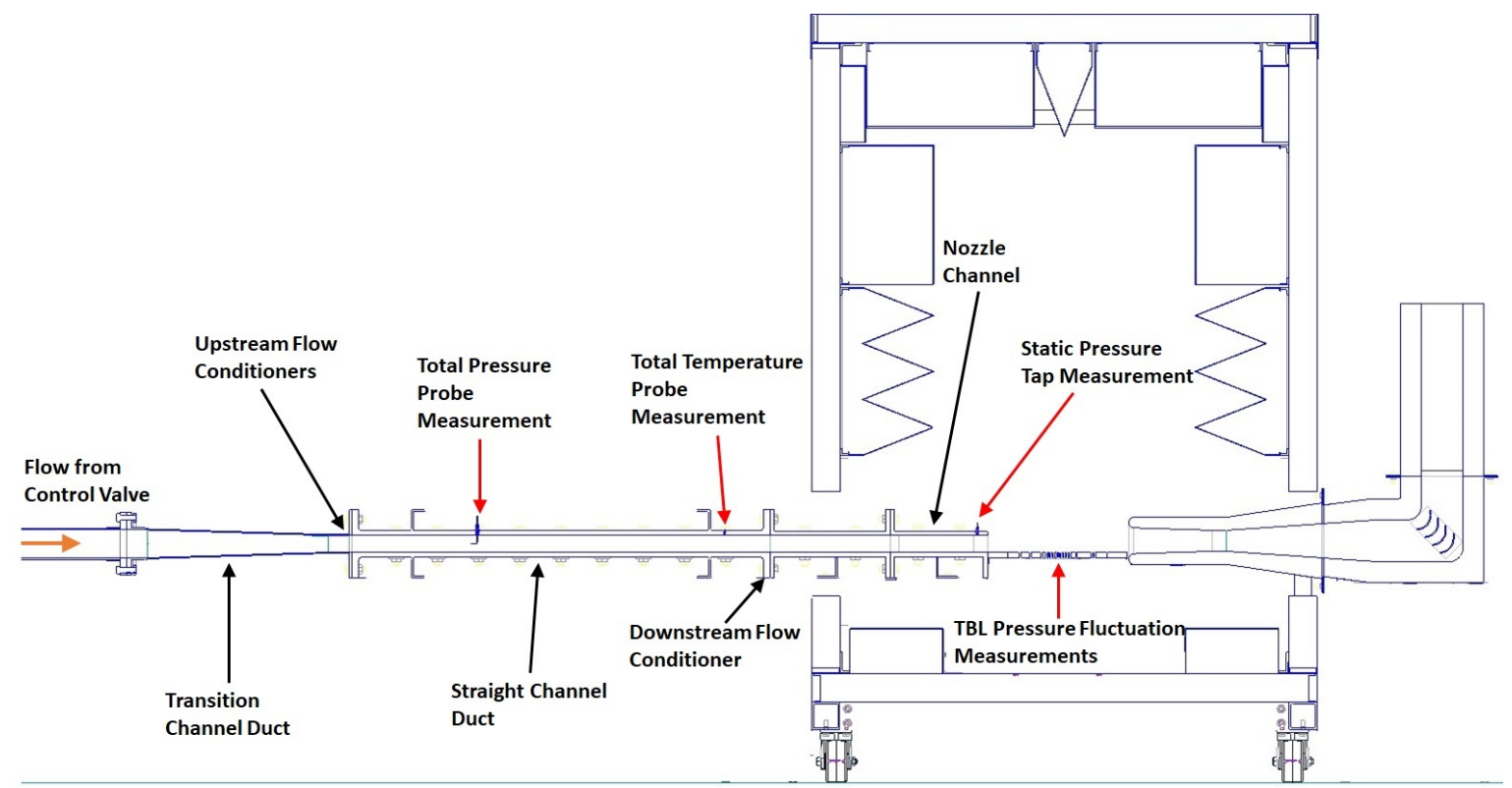

Figure 4.2: Schematic of wind tunnel measurement system locations.

The static pressure tap was drilled on the top surface of the nozzle channel just before the orifice where the Mach number control point is desired. The total pressure 
measurement is conducted using a fabricated pitot probe which is designed to feed

through the top of straight channel assembly and immersed within the centreline of the flow. Its placement, approximately $40.64 \mathrm{~cm}$ downstream of the upstream flow conditioners, ensures that the probe is not exposed to significant levels of inhomogeneous turbulence produced in close proximity to the screen based on isotropic grid turbulence decay theory [84]. A thermocouple used to measure the total temperature is placed just upstream of the second flow conditioning screen in a similar manner as the total pressure probe. All of the aforementioned measurement systems interface with a wind tunnel control and Data Acquisition (DAQ) system already established in the facility; with the exception of the pressure fluctuation sensors. The sections to follow discuss this control system, as well as all of the utilized instrumentation in more detail.

\subsection{Existing Facility Control System Hardware}

Figure 4.3 contains the complete system block diagram of the existing wind tunnel control system; with the last design iteration performed by Hall [50].

Implemented pressure transducers in the wind tunnel are wired into a National Instruments SCB-68 shielded I/O connector block. The connector block is connected to a 16 bit National Instruments PCI-6229A DAQ card mounted in a desktop computer within the control room running Windows XP Professional with 2 GB of ram and an Intel Pentium $43.0 \mathrm{GHz}$ processor. A PID control system algorithm, written in LabVIEW 8 software by Hall [50], is used to compute the required signal response to the control valve based on the input pressure voltage data and selected test section target Mach number. The voltage control signal, kept within the range of $0-10 \mathrm{~V}$, is fed to an Omega OM7-39-02-C voltage-to-current converter, which converts the 


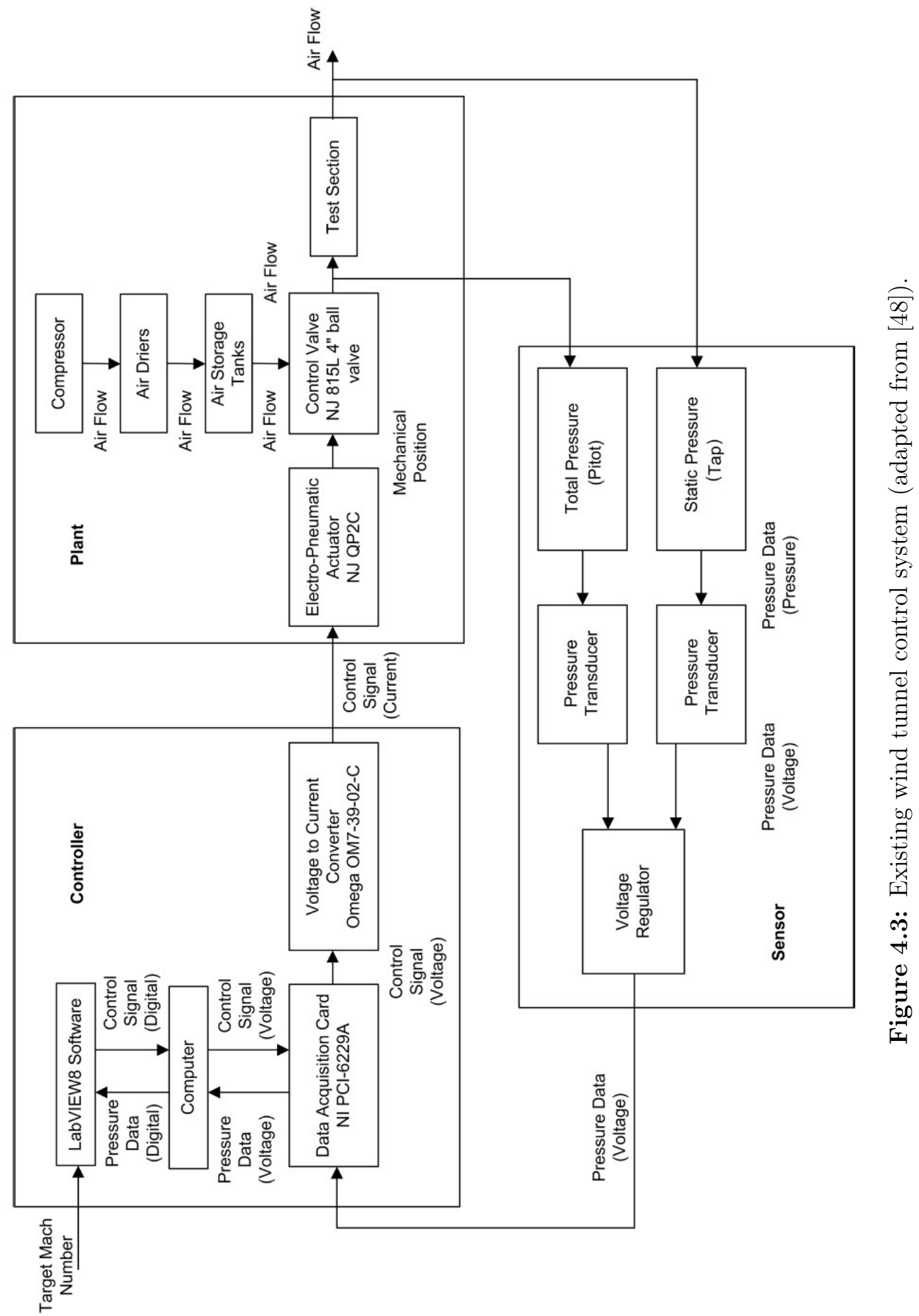


signal to a regulated scaled current output between 0-20 mA. The current output is sent to the valve positioner (discussed in Chapter 1.2.3) to govern the position of the valve to complete the feedback loop.

In addition to the main analog voltage DAQ card, the control room also contains a National Instruments 24 bit USB-9211A thermocouple DAQ unit. This unit is capable of 3 direct thermocouple inputs with compatibility with multiple thermocouple types. The system contains built-in cold-junction compensation and signal conditioning to obtain a direct calibrated temperature measurement. This system is connected to the control computer via USB and synchronized within the control system software.

An Omega PX613-150G5V 0-150 psig pressure transducer, fixed to one of the storage tanks via a NPT threaded adapter, monitors the tank pressure and displays the calibrated value on an Omega TX82B digital indicator inside the control room.

\subsection{Wind Tunnel Instrumentation}

Along with the control system hardware and sensors discussed in the previous section, the HSAWT was fitted with additional sensors and DAQ equipment to facilitate proper operation and data collection. This includes the use of a new pressure sensor array; since the previous set was removed and re-purposed for another project (except for one reference absolute pressure transducer). The new equipment also consists of a video camera for test section monitoring and the movable DAQ system for the pressure fluctuation sensors used previously in the Carleton MSAWT facility [17].

Figure 4.4 contains the full instrumentation system diagram for the HSAWT. The sub-sections to follow describe the additional instrumentation system equipment in more detail. 


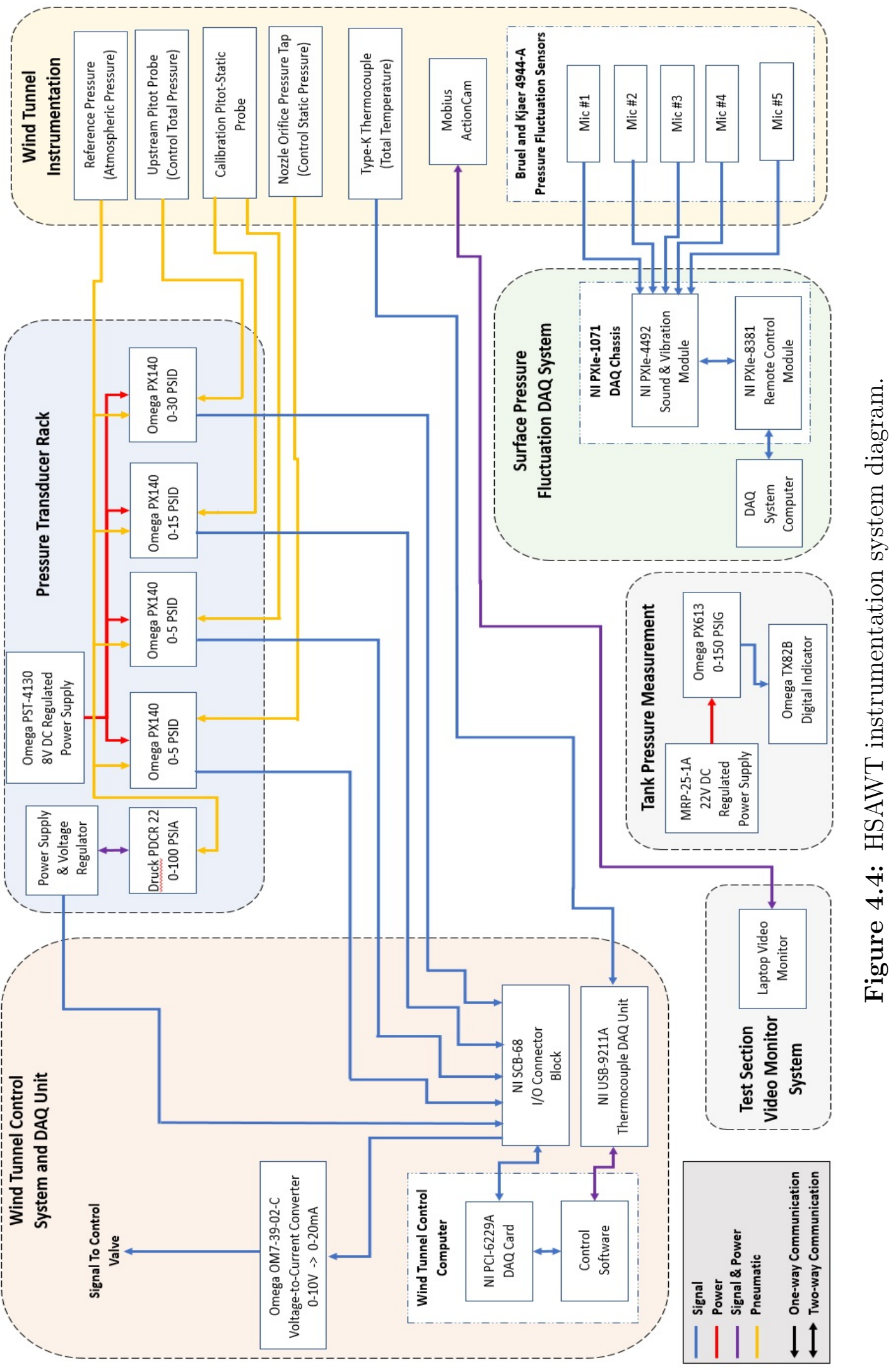




\subsubsection{Control Total Pressure Pitot Probe}

The pitot probe used to measure the total pressure upstream of the test section for control is shown in Figure 4.5 .

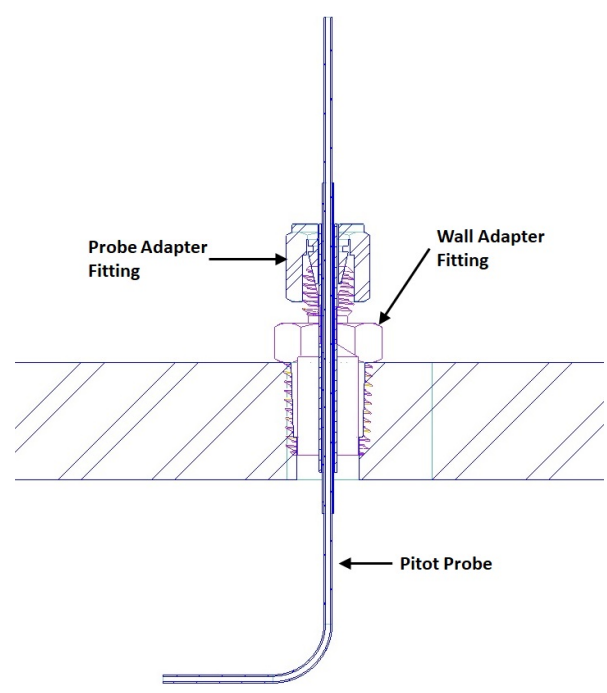

(a) Section schematic view

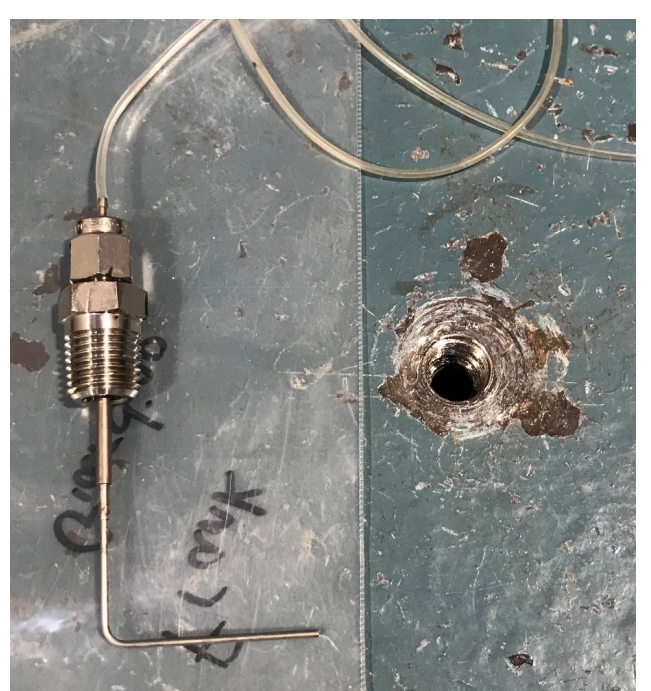

(b) Fabricated probe

Figure 4.5: Control total pressure pitot probe.

The probe was fabricated from 18 gauge $(1.27 \mathrm{~mm}$ O.D.) hypodermic stainless steel tubing with a $90^{\circ}$ bend. A second straight piece of tubing was epoxied over the portion of the main tubing which comes in contact with the adapter fitting to provide extra bending strength. The probe adapter fitting contains metallic ferrules that grip the probe. The probe adapter threads onto a second adapter which threads into the wall surface via a $1 / 4$ "-NPT connection. The probe tip distance from the main stem is $19.05 \mathrm{~mm}$. The probe centreline alignment error is within $\pm 2.5^{\circ}$; well below the suggested maximum allowable misalignment causing significant pressure measurement errors $\left( \pm 10^{\circ}\right.$ according to literature for this specific probe tip geometry [76, 83]). 


\subsubsection{Control Static Pressure Tap}

The static pressure tap used for control is displayed in Figure 4.6

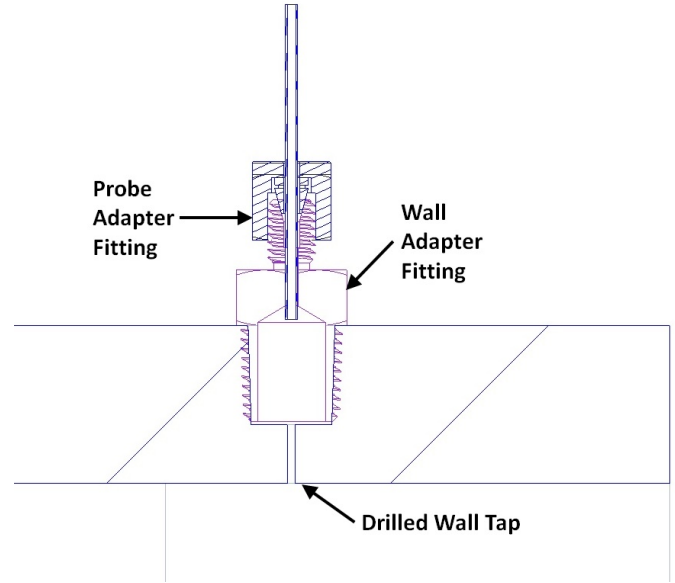

(a) Section schematic view

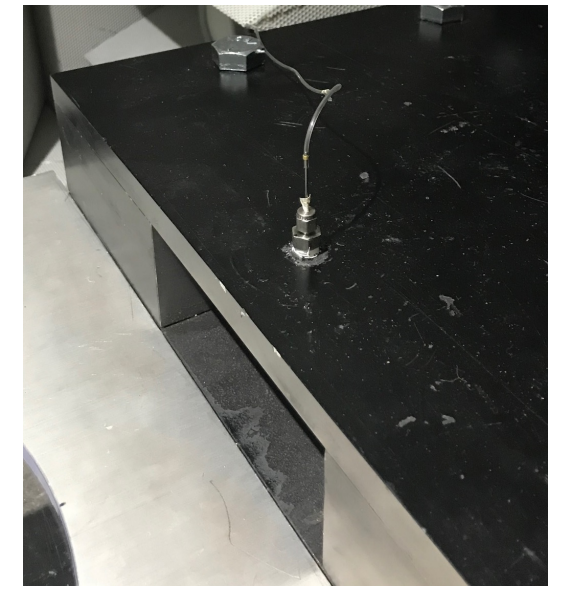

(b) Fabricated tap placement

Figure 4.6: Control static pressure tap.

The wall tap sits at a centreline distance of $3.81 \mathrm{~cm}$ from the nozzle orifice with a diameter of $0.75 \mathrm{~mm}$. The tap depth-to-diameter ratio is roughly 8 , which is slightly above the suggested value of 5-6 in literature to avoid slight pressure errors (positive in nature and roughly between 1-2\%) [83. A similar wall adapter fitting with small steel tubing is placed over the tap so that the pneumatic pressure line can be fed to the transducer.

\subsubsection{Calibration Pitot-Static Probe}

A United-Sensor Corp. pitot-static probe used to calibrate wind tunnel flow speeds for the MSAWT set-up [17] was selected for test section total and static pressure calibrations in comparison with measurements from the fabricated systems. 


\subsubsection{Reference Absolute Pressure Transducer}

A Druck PDCR 22 0-100 psia absolute pressure transducer is used to provide a reference pressure measurement for the differential pressure transducers in the HSAWT. This reference is nominally the atmospheric conditions in the facility just outside of the test chamber. The transducer is connected to a regulator unit which provides a regulated excitation and millivolt output to the control system DAQ. Jeffries [47] determined that the transducer posses a combined voltage repeatability and hysteresis of $\pm 0.25 \%$ of the reading. Calibration information is detailed in Chapter 5.1 .

\subsubsection{Differential Pressure Transducers}

Four Omega PX140 series differential pressure transducers are used to measure important pressures within the tunnel circuit (pressures for tunnel control and flow calibrations). The technical details of these transducers are provided in Table 4.1 .

Table 4.1: HSAWT differential pressure transducer data 85].

\begin{tabular}{cccc}
\hline Transducer & $\begin{array}{c}\text { Range } \\
\text { [psid] }\end{array}$ & Measurement & $\begin{array}{c}\text { Repeatability/Hysteresis } \\
\text { Error [\%FS Voltage Range] }\end{array}$ \\
\hline \hline PX142-005 & $0-5$ & Static Tap & $\pm 0.25 \%$ \\
PX142-005 & $0-5$ & Calibration Static & $\pm 0.25 \%$ \\
PX142-015 & $0-5$ & Calibration Total & $\pm 0.15 \%$ \\
PX142-005 & $0-5$ & Upstream Total & $\pm 0.15 \%$ \\
\hline
\end{tabular}

The transducers measure a differential pressure relative to the reference pressure transducer. The transducer output voltage spans linearly between 1 to $6 V D C$ for a regulated excitation of $8 V D C$. The supply voltage is provided by an Omega PST4130 regulated power supply. Calibration information for all of the transducers is provided in Chapter 5.1 . 


\subsubsection{Total Temperature Thermocouple}

The flow total temperature is monitored by a type-K thermocouple immersed within the flow using a similar adapter to the one used for the pitot probe. The thermocouple is connected to extension wire leads which connect directly to the thermocouple DAQ unit inside the control room. The DAQ unit and control system software is designed to output the temperature in calibrated units with cold-junction compensation.

\subsubsection{Surface Pressure Fluctuation Sensors}

The sensors used to measure the TBL surface pressure fluctuations consist of five high performance Bruel and Kjaer (B\&K) 4944-A, 1/4" pressure field microphones with built-in DeltaTron preamplifiers. The microphones are nominally fitted with grid caps over the sensor diaphragm for free-field acoustic measurements, however, they can be replaced with flat caps that contain a small $0.5 \mathrm{~mm}$ pinhole at the centre to facilitate flush mounted surface pressure fluctuation measurements with a dynamic frequency range of 16 to $70,000 \mathrm{~Hz}$. The absolute measurement uncertainty of each transducer is $\pm 1.035 \mathrm{~Pa}(0.3 d B$ rel. $1 \mathrm{~Pa})$. Each microphone was last calibrated by VanBlitterswyk [17] who confirmed the manufacturer provided sensitivities which are tabulated in Table 4.2 .

Table 4.2: Microphone sensor calibration data [17].

\begin{tabular}{crc}
\hline Microphone Number & Serial Number & Sensitivity $(\mathbf{m V} / \mathbf{P a})$ \\
\hline \hline 1 & 2931836 & 0.830 \\
2 & 2931839 & 0.819 \\
3 & 2931840 & 0.897 \\
4 & 2931837 & 0.851 \\
5 & 2931838 & 0.848 \\
\hline
\end{tabular}


Figure 4.7 contains a schematic of the microphone, including the method of flush mounting the sensor to the acrylic test panel using removable adhesive clay on the bottom surface of the panel. Figure 4.8 shows a photo of the test section panel depicting a common sensor mounting configuration within the panel. All unused mounting holes in the test panel are filled using blue silicone plugs.

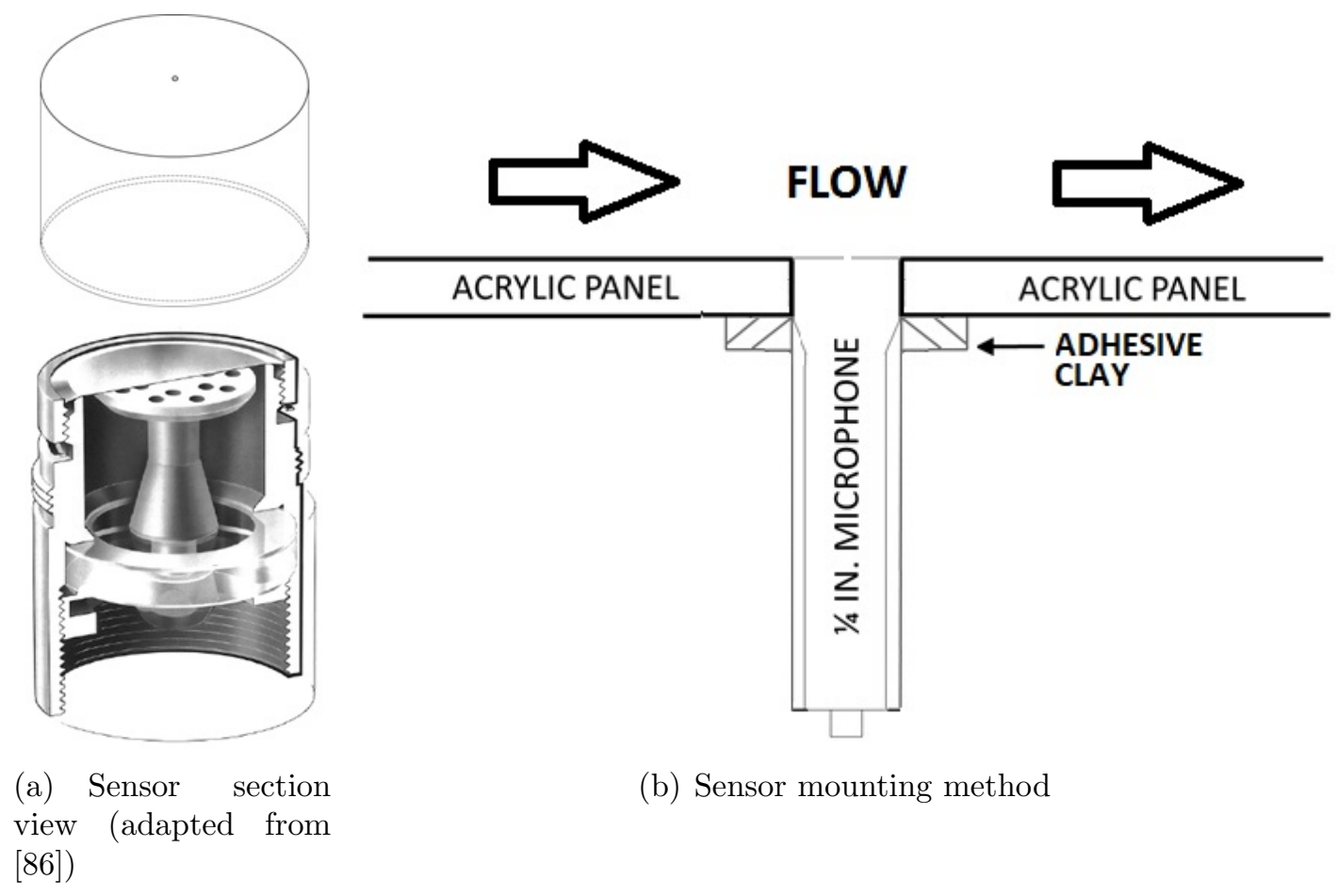

Figure 4.7: Fluctuating pressure sensor schematic.

The microphone data is acquired by a separate DAQ system which utilizes a 24 bit, 8 channel National Instruments PXIe-4492 Sound and Vibration module housed in and powered by a PXIe-1071 DAQ chassis. The module is specifically designed for acquiring sensors with a large dynamic range at high sampling rates. The chassis and module is also designed to supply an excitation current to the amplifiers within the microphones through AO 0587-D-100 shielded cables. The chassis and module interface with a dedicated DAQ computer running Windows 7 with 16 GB of ram and 


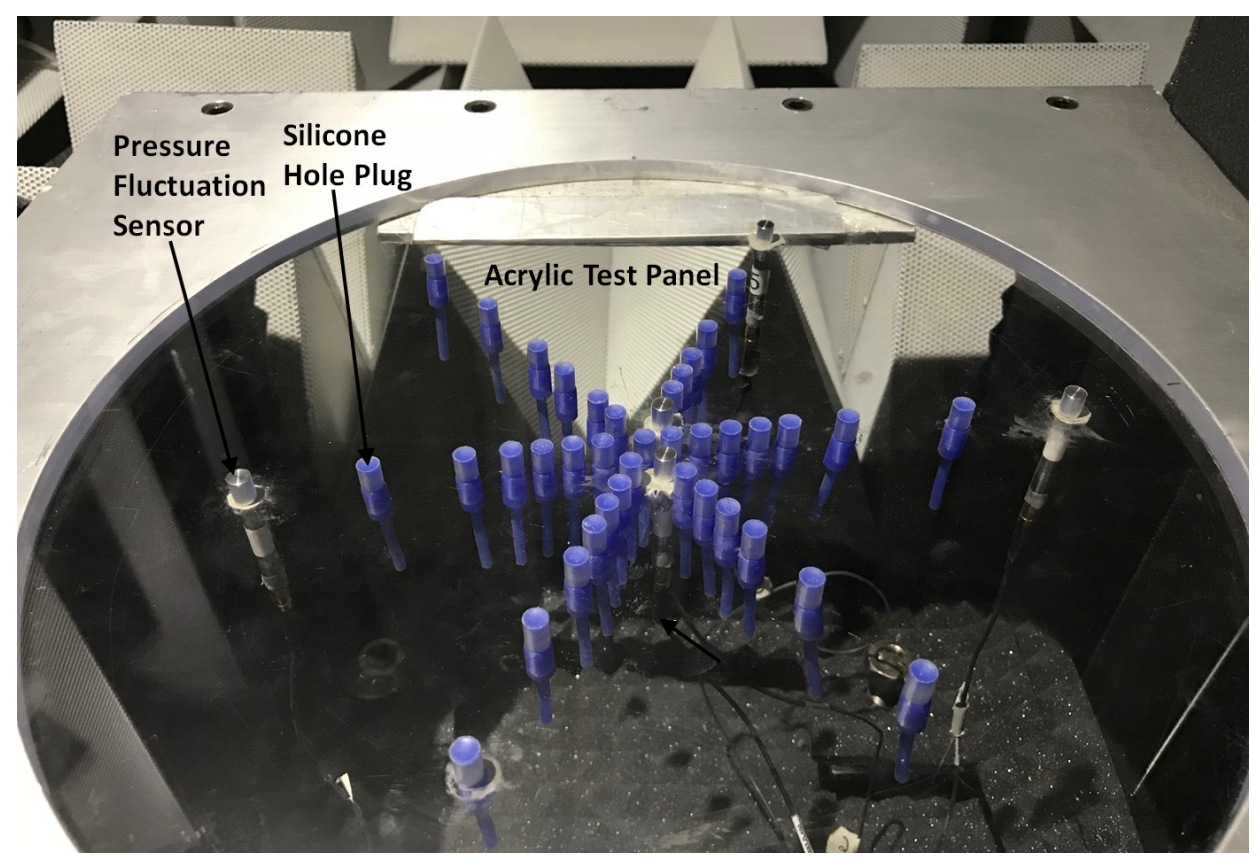

Figure 4.8: TBL test panel sensor flush mounting.

an Intel Core i7-4770 3.4 GHz processor using a PXIe-8381 Remote Control Module. The data is recorded using software written in LabVIEW 13. A discrete trigger event and other post-processing methods are used to synchronize the microphone data with the wind tunnel pressure data from the control system DAQ.

\subsubsection{Test Section Video Camera}

A Mobius ActionCam video camera is mounted in the test chamber to obtain a live video feed of the test section during use of the wind tunnel (mounted on one of the wall wedges and pointed at the panel as seen in Figure 3.20. The camera is capable of recording video in 1080p resolution and connects directly to any laptop computer via a USB adapter for live video display. 


\section{Chapter 5}

\section{Calibrations and Wind Tunnel Commissioning}

This chapter presents the calibrations and control system parameter tuning performed for the commissioning of the HSAWT. This includes details regarding sensor and test section flow speed calibrations; control system software revisions; and measured flow data results with uncertainty estimations.

\subsection{Sensor Calibrations}

All pressure transducers used in the wind tunnel were calibrated for direct output in engineering units (psi) by fitting a linear function to an averaged set of calibration data (3 sets max). Calibrations were performed using a Druck DPI 605 precision portable pressure calibrator. This calibrator is capable of generating positive and vacuum pressure inputs to a connected transducer with an accuracy of $0.025 \%$ of the reading. Differential pressure transducer calibrations were all conducted with the reference port open to atmosphere in the control room. The voltage response of the transducers was recorded using the control system DAQ software. The calibrations of each transducer are integrated into the program for the control system. 
Calibration of the reference absolute transducer followed a linear function of the measured voltage, $V$, in the form of:

$$
P=A_{c a l} V+B_{c a l}
$$

Figure 5.1 contains the plot of the calibration data which was performed for the general expected range of use for the transducer (around atmospheric conditions).

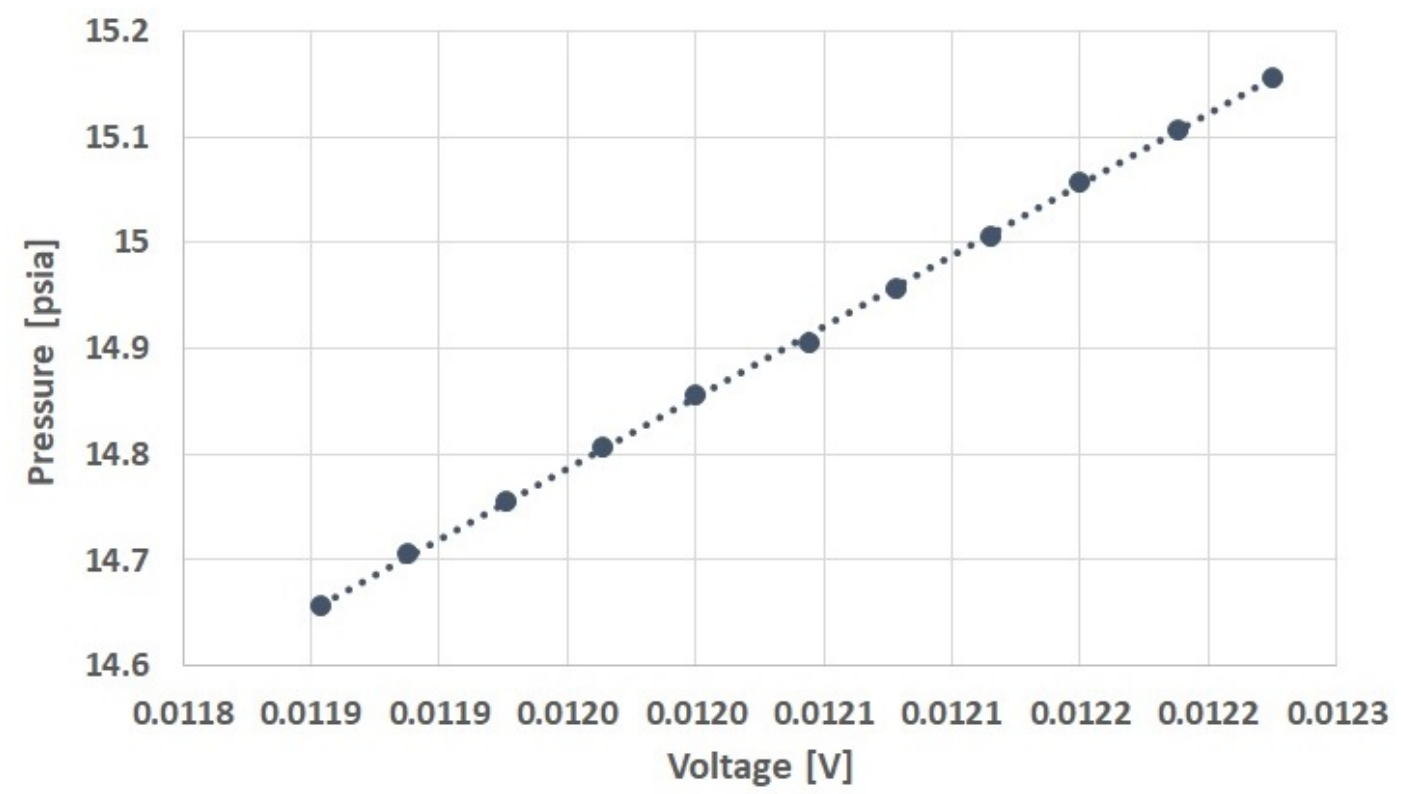

Figure 5.1: Reference pressure transducer calibration curve.

The differential transducers were calibrated over their expected pressure range of use as well. However, the calibration curves were performed based on the differential pressure output as a function of the measured differential voltage with respect to the zero voltage reading. The reason for this approach is that the zero reading tends to fluctuate about its nominal value on a day-to-day basis due to varying atmospheric conditions in the facility. The linear calibration fit thus becomes:

$$
P_{\text {diff }}=A_{\text {cal }}\left(V-B_{\text {offset }}\right)
$$


where the zero offset, $B_{\text {offset }}$, is determined and corrected for via daily measurements before the wind tunnel is run. Figure 5.2 contains the calibration data for the differential voltage measurements and their corresponding differential pressure outputs for all four transducers. The curves for the two 5 psid transducers essentially overlap each other. Table 5.1 summarizes the calibration coefficient data obtained from the regression analysis.

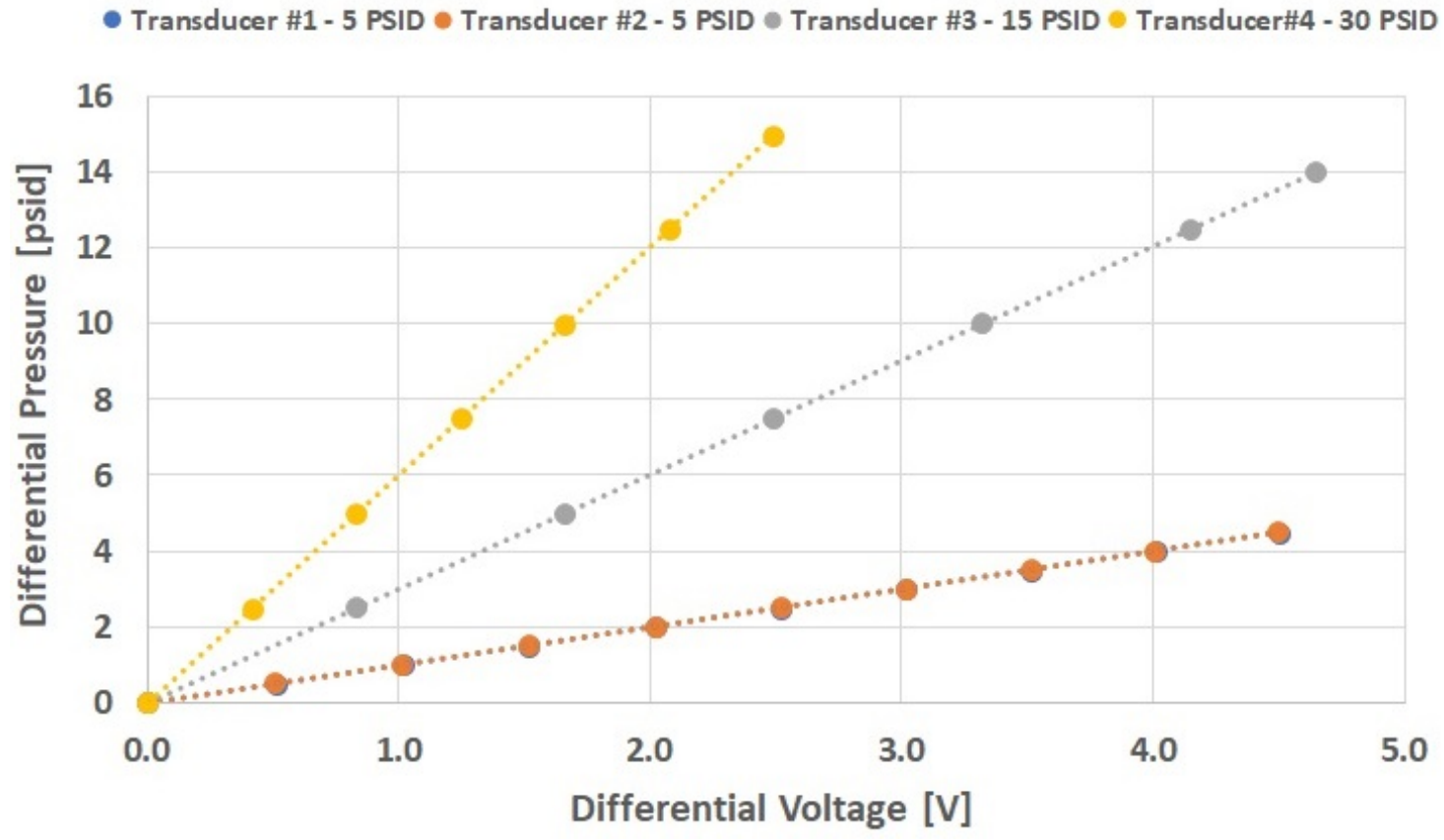

Figure 5.2: Differential pressure transducer calibration curves.

Table 5.1: Pressure transducer calibration data.

\begin{tabular}{cccc}
\hline Transducer & Serial Number & $A_{\text {cal }}$ & $B_{\text {cal }}\left(B_{\text {offset }}\right)$ \\
\hline \hline Ref. Absolute $(\# 0)$ & 76741 & 1338.83 & -1.21214 \\
Diff. 5 psid (\#1) & PX142-005 & 0.9958 & measured daily \\
Diff. 5 psid (\#2) & PX142-005 & 0.9961 & measured daily \\
Diff. 15 psid (\#3) & PX142-015 & 3.0126 & measured daily \\
Diff. 30 psid (\#4) & PX142-030 & 6.0284 & measured daily \\
\hline
\end{tabular}




\subsection{Control System Parameters}

The control system software developed by Hall [50], was modified for the current HSAWT instrumentation and set-up. The primary PID control algorithm however is still implemented in the software. The following sub-sections describe some of the important software parameters that were utilized during commissioning of the wind tunnel.

\subsubsection{Sampling Parameters}

Due to the high test section speeds involved, it is important to select adequate sampling parameters for the pressure transducer data to ensure that proper steady-state control conditions are obtained. The control system is designed to obtain a fixed set of pressure data points at a specified sampling rate, compute the average value of each data set, propagate that value in the Mach number calculation and control system algorithm to compute a valve control response. Jeffries [47] conducted an analysis for the previous tunnel configuration to determine the proper sampling parameters for control. He observed fluctuating pressures in the mean flow up to $1000 \mathrm{~Hz}$, which accounting for the Nyquist criterion, resulted in the selected sampling rate of 2000 $\mathrm{Hz}$ to resolve the complete content of the signals. He also determined that averaging samples over a period of $0.1 s$ was sufficient to obtain statistical convergence on the mean pressure of the measurement set. These parameters were therefore selected as the baseline sampling parameters for this study. Due to the use of different sensors and wind tunnel components however, these parameters may not be optimal and it is recommended that they be determined through a more extensive study for this specific set-up in the future (refer to Chapter 7). 


\subsubsection{Control System Gains}

The gains for the proportional, integral, and derivative portions of control system can be modified by the user depending on the type of desired Mach number control

response. In general, the proportional gain, $K_{p}$, is used to obtain a desired settling time in the controlled response. An increase in the gain will tend to decrease the settling time at the expense of the possible onset of oscillations. A value of 9 was selected for the HSAWT which is slightly higher than the values used by Hall [50] and Kibsey [48] $\left(K_{p} \sim 6-8\right)$ for the old tunnel configuration. This value provided a more reasonable settling time for the control response.

An increase in the integral gain, $K_{I}$, is used to help reduce steady-state error in the control response (found to be a Mach number undershoot in nature), at the expense of increased steady-state oscillations. Hall [50] recommended the selection of a $K_{I}$ value relatively close to the selected $K_{P}$. A $K_{I}$ value of 9 was selected which produced a reasonable response at most flow conditions.

Finally, an increase in the derivative gain, $K_{D}$, is used to help decrease steadystate oscillations, however doing so by increasing the settling time. A value of 2 for $K_{D}$ was used as recommended by Hall [50] and Kibsey [48].

It should be noted that the values of the control gains were selected and kept as the baseline parameters for commissioning. No extensive control gain parameter sensitivity analysis was conducted to obtain optimized gains in terms of the control response for various test section governed Mach numbers. It is recommended that this study be conducted in the future as it may lead to longer steady controlled run times (Chapter 7). 


\subsubsection{Control Valve Initial Threshold Voltage}

In practice, the control valve requires some initial voltage input to start to obtain flow through it. This is most likely due to some required angle that the ball valve must rotate to start to obtain flow across the body. During initial testing, the supply voltage output was monitored at the lowest test section governed speed $(\mathrm{M}=0.1)$, and the initial threshold voltage was determined to be roughly $1.25 \mathrm{~V}$. The control system was modified to send that value as the initial supply output to obtain a quicker control response during start-up.

\subsubsection{Control Mach Number Undershoot}

A characteristic of the wind tunnel, both observed for the HSAWT as well as the previous configuration by Hall [50], is a pronounced undershoot in the governed test section Mach number in comparison to the specified target. Table 5.2 contains a list of the controlled Mach number undershoot percentages with respect to the specified target found during preliminary testing. These values are based from averaged sets of conducted measurements.

Table 5.2: Control Mach number undershoot behavior.

\begin{tabular}{ccc}
\hline Control Mach Number & Undershoot [absolute] & Undershoot [\%] \\
\hline \hline 0.1 & 0 & 0 \\
0.2 & 0.003 & 1.5 \\
0.3 & 0.006 & 2 \\
0.4 & 0.010 & 2.5 \\
0.5 & 0.014 & 2.8 \\
0.6 & 0.019 & 3.17 \\
0.7 & 0.026 & 3.71 \\
0.8 & 0.035 & 4.375 \\
\hline
\end{tabular}


The observed undershoot becomes more pronounced as the test section Mach number is increased. Hall [50] provides a description of the phenomena and recommends the use of a direct correction factor to modify the set target Mach number to remove the behavior rather than attempt to reduce it using the integral control gain, $K_{I}$. This is because the required control gain to remove the undershoot results in a response with unstable oscillations [50].

\subsection{Flow Speed Calibrations}

As described is Chapter 4, reference pressure measurements used for control of the Mach number must be corrected for their true values at the point of control (nozzle exit). This is performed using the calibration pitot-static probe for control of the wind tunnel while monitoring the respective total and static pressure outputs of the upstream fabricated pitot probe and nozzle static tap. Calibration curves can then be constructed for future control of the wind tunnel using the reference pressure measurements. Calibration measurements were repeated several times on different days and atmospheric conditions to ensure the results were within an acceptable degree of repeatability $( \pm 0.5 \%)$.

Figure 5.3 contains calibration data for the total pressure measurements between the upstream reference pitot, $P_{o_{r e f}}$, and the calibration probe at the nozzle exit, $P_{o}$, for various test section Mach numbers. The absolute pressure difference between the two measurements is outlined in Figure 5.3(a). The data shows an expected increasing trend in the pressure loss between the two points as the Mach number is increased, due to the second flow conditioning screen and duct friction losses. It is important to note that some portion of the difference in pressure may likely be due to geometric differences between the fabricated probe and the calibration probe. The 
plot in Figure 5.3(b) displays the gauge pressure ratio, $P_{o_{r e f}} / P_{o}$, which represents the correction factor that is directly applied in the control system software to the reference probe measurements to obtain the proper pressure for control. A ratio based on the direct calibrated output of the respective differential transducers [psig] was selected instead of using a ratio based on the atmospheric corrected absolute measurements to avoid the propagation of error from the reference transducer output.

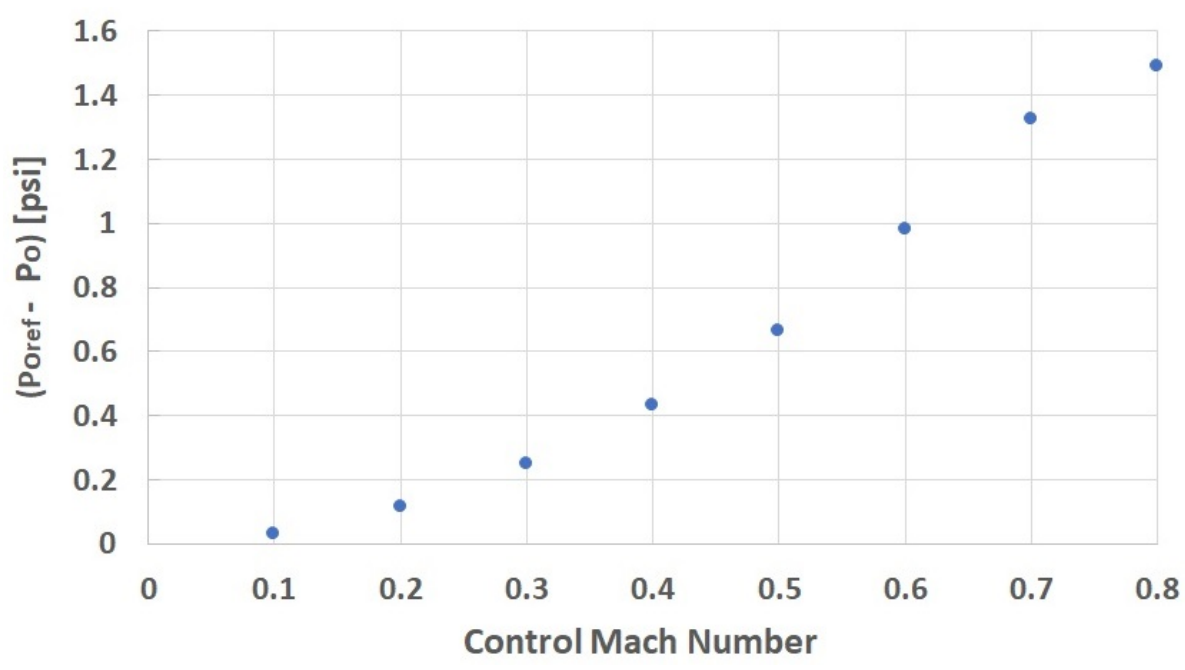

(a) Total pressure difference calibration

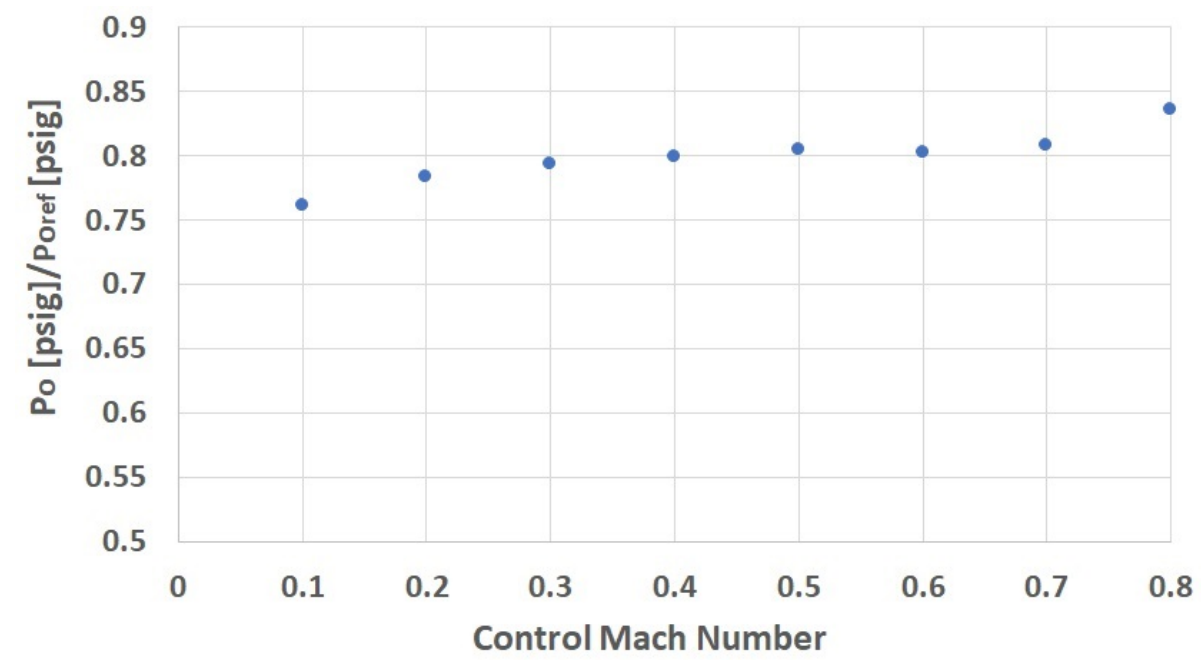

(b) Gauge pressure ratio calibration

Figure 5.3: Control total pressure calibration data. 
Since the differences between the static pressure measurements are small, the comparisons between the value at the tap, $P_{\text {ref }}$, and the calibration probe, $P$, were made based on the absolute error. This value is directly influenced by the tap geometry because they are measured at the same location. Figure 5.4 contains the measured static pressure error data, which displays the expected positive error trend seen in literature for the implemented tap geometry [83. The measurements at the tap are corrected directly in the control algorithm using these error values for the respective selected control Mach number.

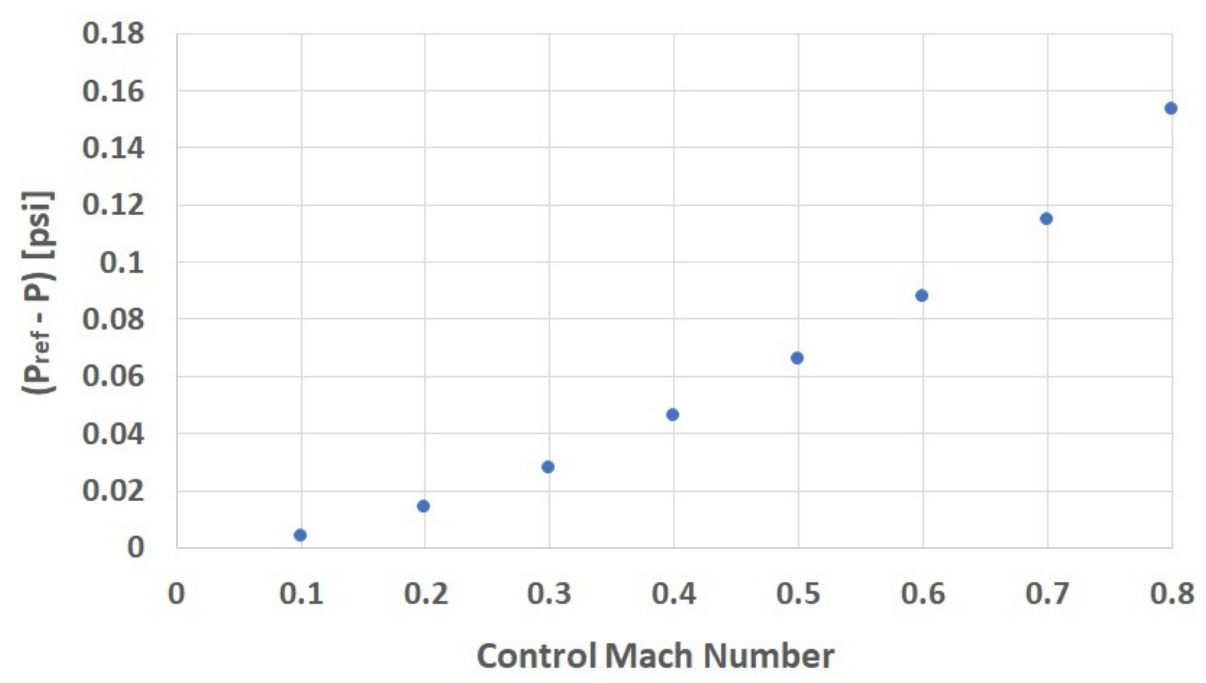

Figure 5.4: Control static pressure tap error correction.

\subsection{Flow Characterization Data}

This section presents some of the important flow data obtained during the primary phase of wind tunnel commissioning. This includes results of test section flow Mach number governed by the control system; as well as other averaged parameters for a typical run such as mass flow rate, run time, and total temperature drop. All data was collected over numerous runs to ensure consistent levels of repeatability. 


\subsubsection{Controlled Mach Number}

Figure 5.5 contains data of the Mach number computed from the control pressure measurements (Equation 4.2) during a typical 50 second run for the entire target flow speed range of the HSAWT. Multiple runs were conducted to ensure that the results contained in Figure 5.5 are repeatable within roughly $\pm 0.25 \%$ for the low speed conditions and $\pm 1 \%$ for the high speed conditions.

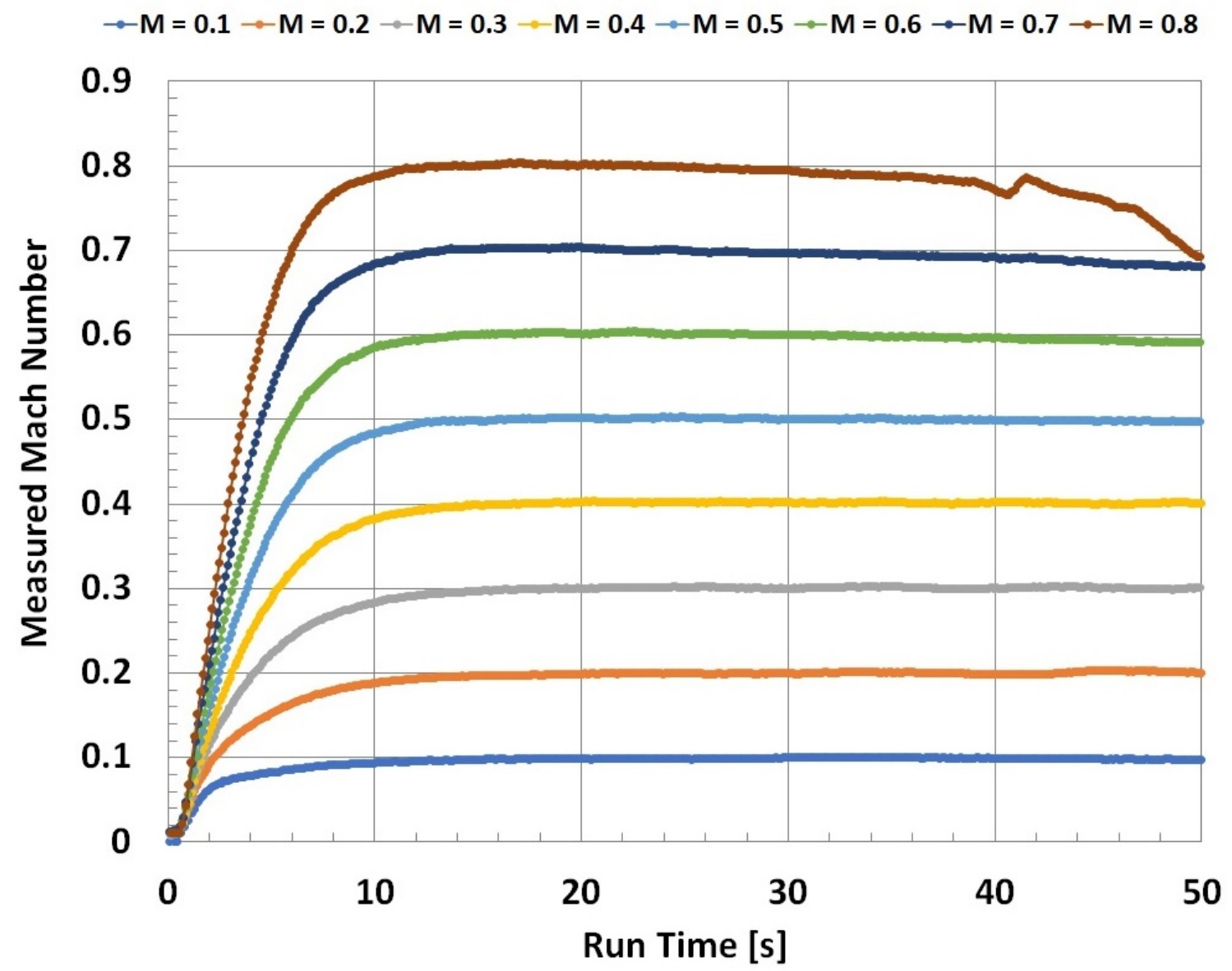

Figure 5.5: Comparison of measured test section Mach number to control system target for a variety of flow speeds.

Looking at the overall data for the implemented control system parameters, the target Mach number is achieved within a settling time of roughly 12 seconds in the 
high speed range and 18 seconds in the low speed range. Constant control of the Mach number for at least 30 seconds is also reasonably achieved for most of the test section range from Mach 0.1 to 0.6 . As the speed is increased to the maximum limit however, the control system response is less steady. This is evident in the response at the maximum control Mach number of 0.8 , where the control valve seems to have a difficult time in compensating for the drop in tank pressure at around 40 seconds.

To obtain a clearer sense of the control system response, Figure 5.6 contains magnified plots of Figure 5.5 for various control Mach number targets for 30 second run time intervals after the response has settled.

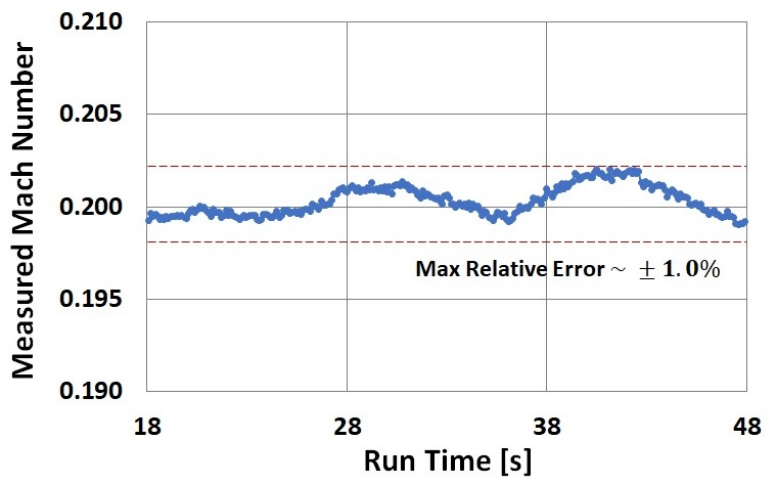

(a) Mach 0.2 target

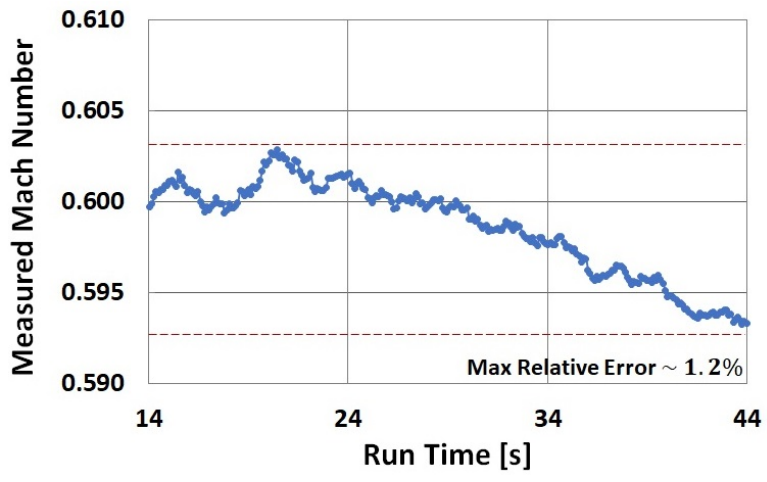

(c) Mach 0.6 target

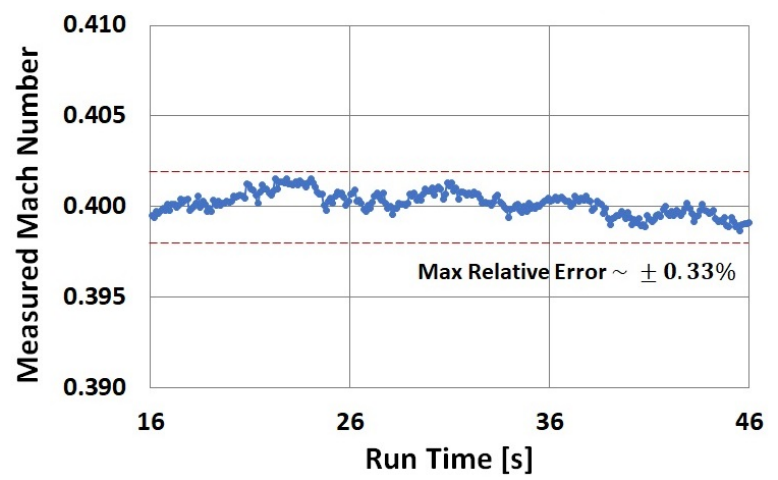

(b) Mach 0.4 target

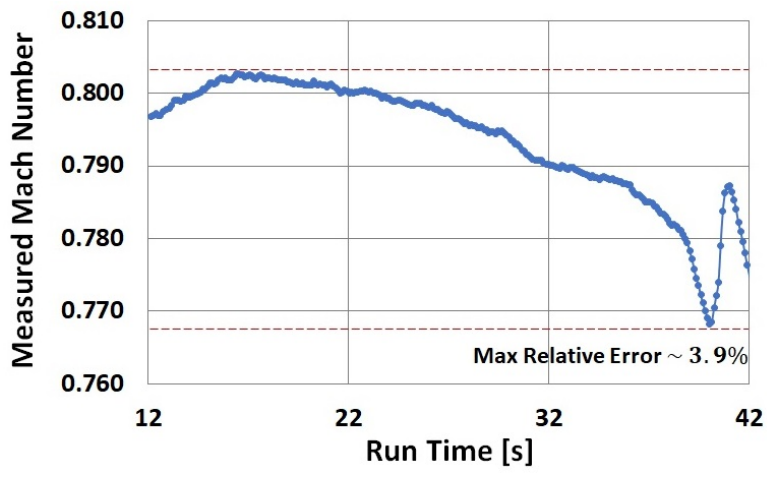

(d) Mach 0.8 target

Figure 5.6: Measured test section Mach number data - Magnified 30 second plots. 
The plots show slight oscillations for the low Mach number range (0.1-0.2); indicative that the selected control system gains and parameters may not be completely optimal for this range. In the mid flow speed range (0.3-0.5) the control system provides relatively good control of the Mach number with a relative error no greater than $\pm 0.4 \%$. For the flow speed range greater than 0.6 however, the control system seems to have difficultly in providing a steady response. The response is also not fluctuating about the target, which indicates that the issue may be more than a matter of selecting proper control system parameters.

Although the maximum relative error is fairly reasonable up to Mach 0.7, the controlled response is slightly unsteady. A possible explanation for this behavior is the mass flow rate demanded from the wind tunnel at the maximum Mach number range is approaching levels that are too high for the control valve to govern steady state conditions downstream in the test section. This implies that the maximum allowable rate at which the control valve can open is not fast enough to match the rate at which the required target valve opening position is increasing. Optimal selection of control system gains may be able to counteract this behavior by providing a fast enough response. The oscillatory 'jump' in the response for the Mach 0.8 data around the 40 second region implies that the valve has a large deadband for this flow condition where the Mach number falls relatively below range before the system tries to compensate. Regardless, introducing slight set-up modifications to lower the required mass flow rate may provide improved control at higher speeds. Chapter 7 discusses recommendations for possible improvements to the system to obtain better control at higher Mach numbers. 


\subsubsection{Mass Flow Rate and Run Time}

The average mass flow rate in a given run is computed using the control pressure data and measurements of the average total flow temperature using Equation 4.1 Since the required mass flow rate mainly dictates the available run time based on the control valve demand from the reservoir tanks, the maximum controlled run time was measured for each flow speed target to compare with the required mass flow rate. The maximum run time was measured at the point at which the difference in the target and calculated Mach number becomes larger than 2.5\%. The results of both are shown in Figure 5.7 .

The mass flow rate at the high test section velocity range is shown to become in excess of $3 \mathrm{~kg} / \mathrm{s}$. This value is quite high in comparison with the flow conditions experienced with the previous wind tunnel configuration [47, 48, 50]. The measured flow rates for the high flow speed range are also higher than the estimated predictions from the preliminary flow analysis conducted during the design stage of the tunnel (refer to Figure 2.9). The reason for the underpredicted levels of mass flow rate may be due to the exhaust system and diffuser components not providing the expected levels

of pressure recovery within the test section, as well as the possible underestimation of flow losses in components such as the flexible high pressure hose, where the onset of friction choking may be occurring at high flow speeds. The implications arise in the available run time, where for Mach 0.8 conditions, is slightly less than the prescribed 30 second target in Chapter 2 . 


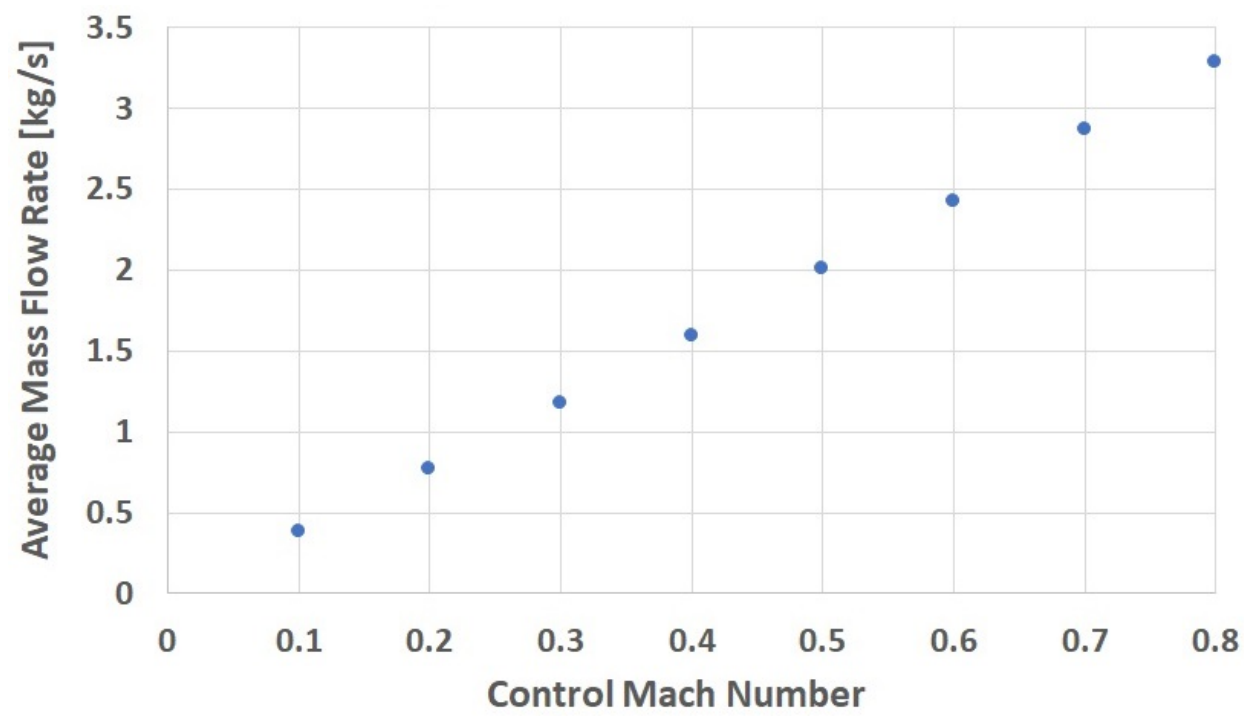

(a) Average test section mass flow rate

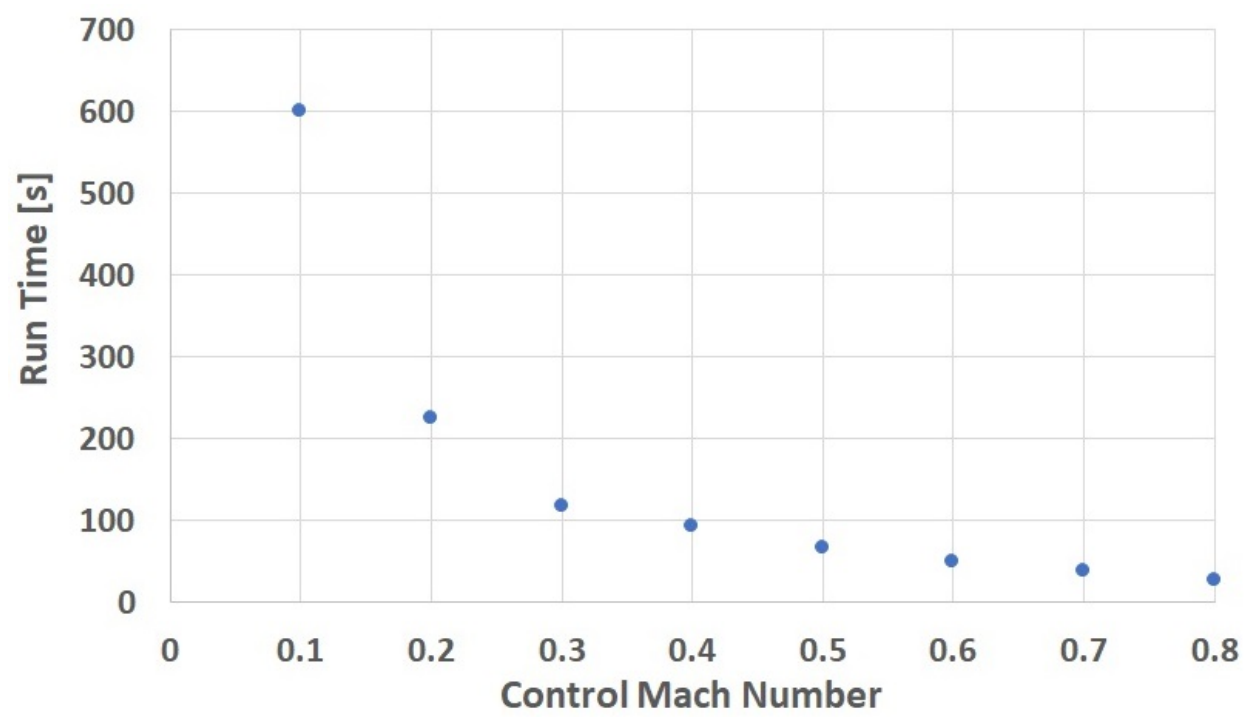

(b) Maximum controlled run time (within $2.5 \%$ Mach error)

Figure 5.7: Wind tunnel test section flow capacity parameters.

\subsubsection{Flow Temperature Drop}

The physical nature of a blowdown facility imposes a drawback of an unsteady decrease in flow temperature as the air is expelled out of the reservoir tanks. This imposes implications on the test section Reynolds number even though the Mach number remains relatively constant. Figure 5.8 contains data of the total drop in 
flow temperature for a typical 30 second run at a given test section speed in terms of the ratio of the final temperature, $T_{f}$, to initial temperature, $T_{i}$, in Kelvin. The data shows that the maximum temperature drop at the highest speeds is roughly $10 \%$.

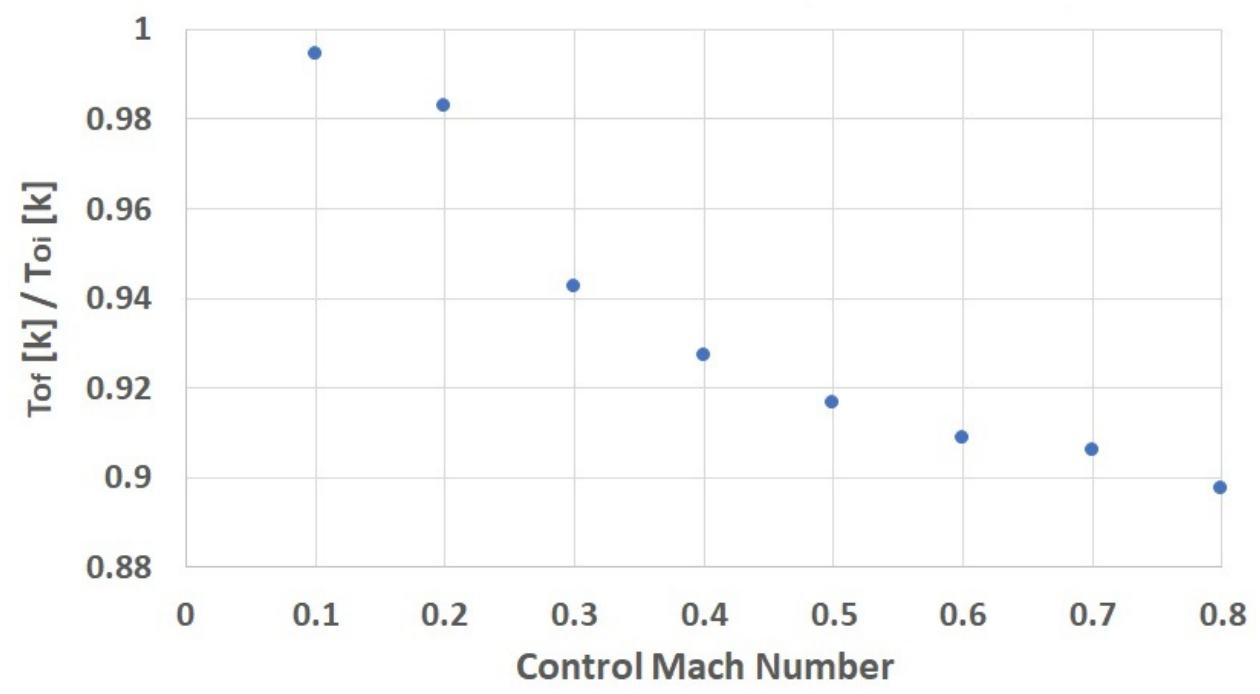

Figure 5.8: Test section total temperature drop (typical 30 second run).

To observe the effects on test section Reynolds number, a relationship for the ratio of the final to initial Reynolds number in a given run, $R e_{f} / R e_{i}$, can be derived in terms of the measured temperature drop ratio. Equation 5.3 displays the relationship between Reynolds number based on some length scale , $l$, and other flow parameters:

$$
R e=\frac{\rho u l}{\mu}=\frac{P(M \sqrt{\gamma R T}) l}{R T \mu(T)}
$$

where the density, $\rho$, and velocity, $u$, can be substituted for compressible flow and ideal gas law relationships.

The viscosity is also dependent on temperature through Sutherland's law [76]:

$$
\mu(T)=\frac{1.458\left(10^{-6}\right) T^{3 / 2}}{T+110.4}
$$


Since all other conditions are constant other than temperature, the ratio $R e_{f} / R e_{i}$ becomes as follows:

$$
\frac{R e_{f}}{R e_{i}}=\frac{T_{i}^{2}}{T_{f}^{2}} \frac{\left(T_{i}+110.4\right)}{\left(T_{f}+110.4\right)}
$$

Figure 5.9 contains the test section Reynolds number variation plot derived from the temperature measurements and Equation 5.5. The figure shows that the temperature drop tends to increase the test section Reynolds number up to a maximum of roughly $35 \%$ for the high speed range.

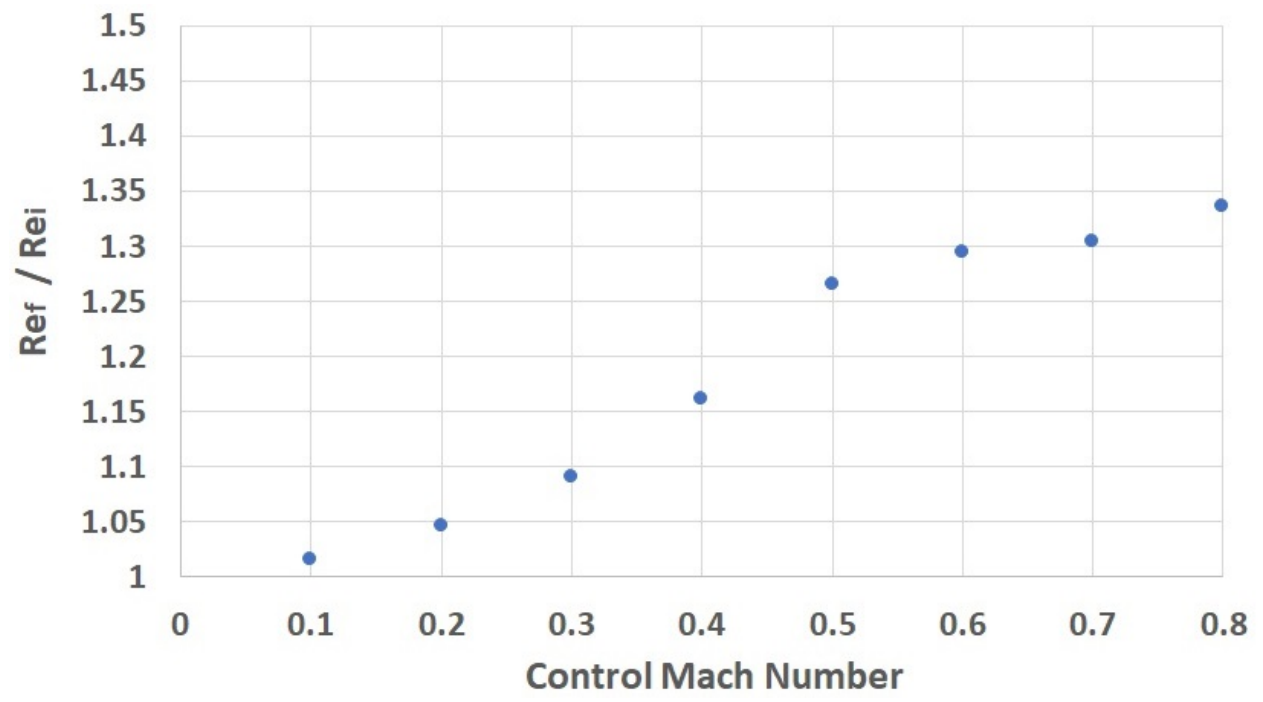

Figure 5.9: Test section Reynold's number variation due to temperature drop (typical 30 second run).

\subsection{Measurement Uncertainty}

It is important to not only examine the uncertainty in the governed control Mach number due to the control system but to also understand the uncertainty in its derivation from measurements of other flow parameters. The sensors used to compute the 
Mach number have inherent uncertainties as well, which propagate through to its computation.

A method outlined by Tavoularis [83] was used to compute the uncertainty in the pressure measurements and the resulting calculation of the Mach number. The method states that the uncertainty in any calculated result, $\delta F$, can be estimated from the uncertainties of the base parameters, $\delta \phi_{i}$, which are used to calculate the computed parameter, $F$, using the following relationship outlined in Equation 5.6.

$$
\delta F=\sqrt{\sum\left[\left(\frac{\partial F}{\partial \phi_{i}}\right)^{2} \delta \phi_{i}^{2}\right]}
$$

where, $\frac{\partial F}{\partial \phi_{i}}$, represents the sensitivities of the computed parameter with respect to each individual base parameter used in its computation.

\subsubsection{Reference Pressure Measurement Uncertainty}

The absolute atmospheric pressure, $P_{a t m}$, measured by the reference pressure transducer is computed in the control system software using the transducer voltage output and the calibration coefficients. This is displayed in Equation 5.7

$$
P_{a t m}=A_{c a l} V+B_{c a l}
$$

Using Equation 5.6, the uncertainty in the reference pressure measurement, $\delta P_{a t m}$, is as follows:

$$
\delta P_{a t m}=\sqrt{\left(A_{\text {cal }}\right)^{2}(\delta V)^{2}+V^{2}\left(\delta A_{\text {cal }}\right)^{2}+\left(\delta B_{c a l}\right)^{2}}
$$


The uncertainty in the voltage output from the transducer was previously discussed as being $\pm 0.25 \%$ of the reading [47. The uncertainty in the calibration coefficients are estimated based on twice the standard deviation in the linear calbration curve defined by the calibration coefficients relative to the actual calibration data points [50]. From these uncertainties, the relative error in the reference pressure measurement was found to be relatively constant for the expected pressure range of use; no greater than $\pm 0.273 \%$.

\subsubsection{Total Pressure Measurement Uncertainty}

The total pressure, $P_{o}$, measured by the differential transducer mentioned in Chapter 4 is computed using Equation 5.9 .

$$
P_{o}=A_{c a l} \Delta V P_{o_{c o r r}}+P_{a t m}
$$

where, $\Delta V$, the is the measured differential voltage relative to the zero offset voltage and $P_{o_{\text {corr }}}$ is the calibration factor applied to the upstream pitot measurement to obtain the true pressure measurement in the test section (Chapter 5.3.

A similar process can be applied to Equation 5.9 to obtain the uncertainty in the total pressure measurement used for the control system, $\delta P_{o}$. Figure 5.10 contains the results from this uncertainty analysis for the entire range of operational total pressures. Since the range of total pressures seen within the wind tunnel is wide, an equation must be fit to the data to estimate the uncertainty in the measurement at a specified operational pressure. This is displayed in Equation 5.10 .

$$
\delta P_{o}=4.2011 P_{o}^{-0.983}
$$




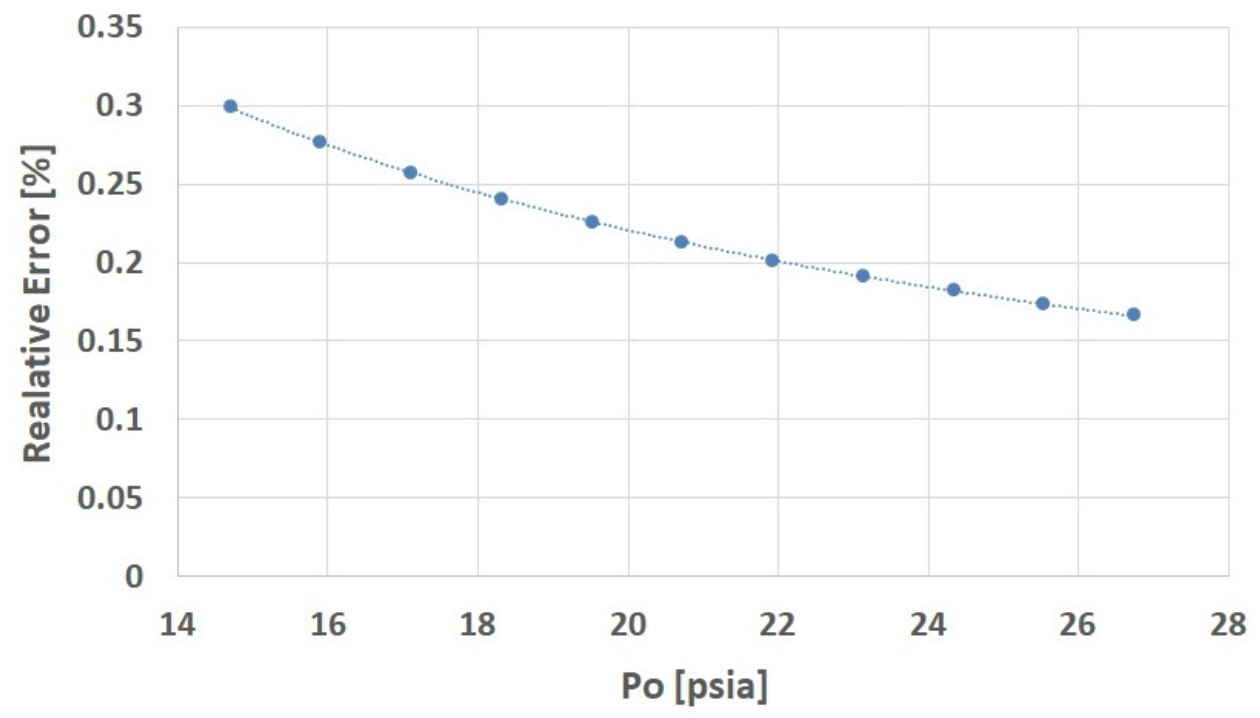

Figure 5.10: Control system total pressure measurement uncertainty.

It is important to note that the error in the voltage measurements for the differential transducers, summarized in Table 4.1, are percentages based on the full scale voltage range $(0-5 \mathrm{~V})$ and not of the reading. This explains the behavior of reduced uncertainty at larger pressure readings.

\subsubsection{Static Pressure Measurement Uncertainty}

The static pressure, $P$, measured by its differential transducer is computed using Equation 5.11 .

$$
P=A_{c a l} \Delta V-P_{e r r}+P_{a t m}
$$

where, $P_{\text {err }}$, is the positive static tap pressure error discussed in Chapter 5.3 . The computed uncertainty, $\delta P$, for its relatively small operational range was found to be relativity constant around $\pm 0.285 \%$. 


\subsubsection{Mach Number Uncertainty}

The Mach number is computed directly from the absolute static and total pressure measurements by rearranging Equation 4.2 to form:

$$
M=\sqrt{\left(\frac{2}{\gamma-1}\right)\left[\left(\frac{P_{o}}{P}\right)^{\frac{\gamma-1}{1}}-1\right]}
$$

The uncertainty, $\delta M$, again is calculated in a similar manner using the previously computed uncertainties of the total and static pressure measurements and the partial derivatives $\partial M / \partial P_{o}$ and $\partial M / \partial P$. Figure 5.11 shows the results for various test section Mach numbers.

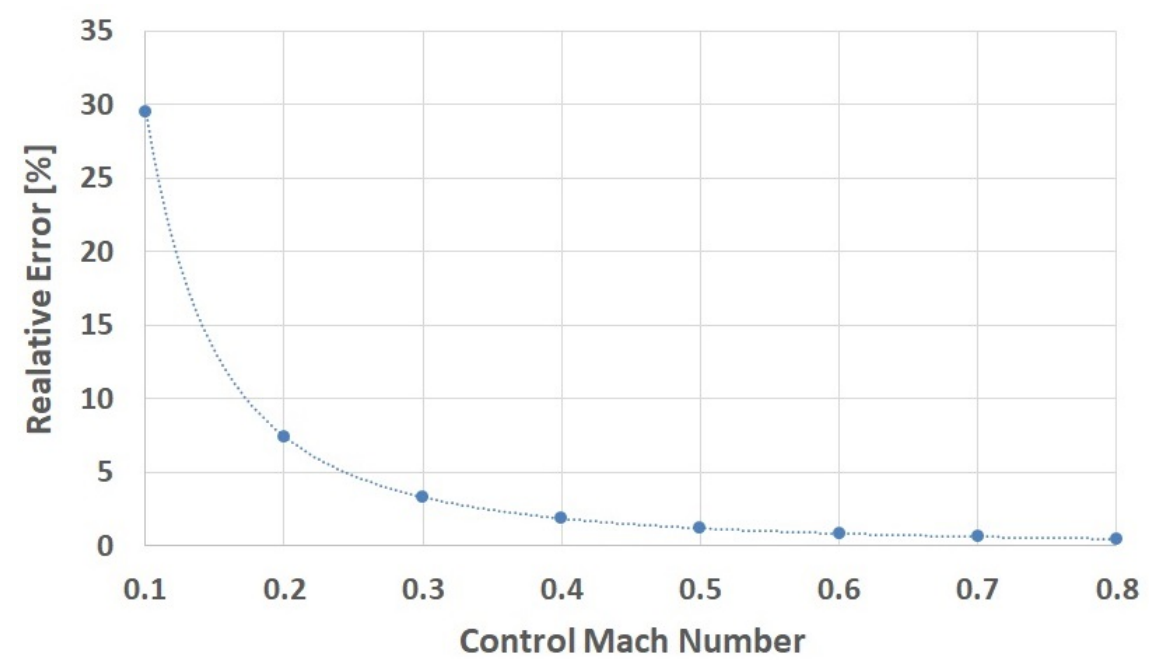

Figure 5.11: Computed control Mach number uncertainty.

The analysis shows that the propagation of error in the pressure measurements is significant at calculations of low Mach numbers due to the large sensitivities in Mach number to incremental changes in low pressure measurements. The relative error becomes more reasonable at Mach numbers greater than 0.3 , with the error becoming $\leq 1 \%$ at Mach numbers $\geq 0.5$. This imposes a restriction on analyzing flows in the low Mach number range, where improvements to the instrumentation, such as 
utilizing more accurate transducers, can alleviate some of the uncertainty at lower controlled Mach numbers. A curve was fit to the data for use as a reference which is displayed in Equation 5.13. Note that is equation is only valid for the observed Mach number range.

$$
\delta M=0.2954 M^{-2}
$$




\section{Chapter 6}

\section{Single-Point Surface Pressure Fluctuation Spectrum Measurements}

The main goal of this research, as discussed in Chapter 1, is to commission the HSAWT facility for TBL pressure fluctuation studies. This chapter discusses the results of the preliminary study conducted regarding measurements of the surface pressure fluctuation spectrum at a single point for comparison with developed prediction models in literature. First, the equations for the spectrum models are outlined, followed by the signal precessing methods used to compute the spectrum from raw sensor data. Results of conducted chamber background noise measurements are also provided to ensure a sufficient signal-to-background noise (SNR) ratio in the spectrum measurements. Finally, the results of the spectrum data are presented and discussed, along with recommendations for future studies.

\subsection{Spectrum Models in Literature}

The following sub-sections describe each model used in comparison with the spectral measurements of fluctuating wall pressure. 


\subsubsection{Robertson Model (1971) [87]}

Robertson derived an early semi-empirical model for predicting the pressure fluctu-

ation spectrum based on the former work of Lowson [88, which relates the PSD of pressure fluctuations to Mach number and other various TBL parameters. Robertson's model addresses some of the issues he found with regards to Lowson's model in terms of inconsistent spectral levels and behavior compared with collected supersonic measurements at NASA-Ames. Robertson proposed a new model shown in Equation 6.1, which corrects for underestimated low-frequency spectral levels and overestimated high-frequency attenuation exhibited by Lowson's model [87].

$$
\Phi_{p}(f)=\frac{(2 \pi)\left(\frac{0.006 q}{1+0.15 M^{2}}\right)^{2}}{\frac{0.5 U_{\infty}}{\delta^{*}}\left[1+\left(\frac{(2 \pi f) \delta^{*}}{0.5 U_{\infty}}\right)^{0.9}\right]^{2}}
$$

\subsubsection{Efimtsov Model (1984) [89]}

This semi-empirical model developed by Efimtsov is based on a number of flight tests and wind tunnel experiments covering a diverse range of Mach numbers and flow conditions [89]. Equation 6.2 presents this model, which displays a principle dependence on flow parameters such as friction Reynolds number, $R e_{\tau}$, and Strouhal number, $S t$, as major contributors to the behavior of the pressure fluctuation spectrum.

$$
\Phi_{p}(f)=\frac{(2 \pi) \alpha \beta u_{\tau}^{3} \rho^{2} \delta}{\left(1+8 \alpha^{3} S t^{2}\right)^{1 / 3}+\alpha \beta R e_{\tau}\left(\frac{S t}{R e_{\tau}}\right)^{10 / 3}}
$$

where:

$$
R e_{\tau o}=3000 ; R e_{\tau}=\frac{\delta u_{\tau}}{\nu} ; \alpha=0.01 ; \beta=\left[1+\left(\frac{R e_{\tau_{o}}}{R e_{\tau}}\right)^{3}\right]^{1 / 3} ; u_{\tau}=U_{\infty} \sqrt{\frac{C_{f}}{2}} ; S t=\frac{(2 \pi f) \delta}{u_{\tau}}
$$

Note that properties such as density and viscosity in Equation 6.2 are representative of the properties of the gas at near wall temperatures, thus Mach number 
effects must be taken into account when calculating these properties from free stream conditions.

\subsubsection{Laganelli Model (1993) [90]}

Laganelli developed a model for the spectrum accounting for viscous, compressibility, and wall heat transfer effects based on influences from the works of Lowson, Robertson, and Blake [27, 87, 88, The model was developed for from both subsonic and supersonic wind tunnel test data and is described in Equation 6.3a where the function $F_{c}$ shown in Equation $6.3 \mathrm{~b}$ is a transformation function from incompressible to compressible flow states.

$$
\begin{array}{r}
\Phi_{p}(f)=\frac{(2 \pi)\left(2.293 \times 10^{-5}\right) q^{2} \delta^{*} F_{c}^{-0.5733}}{U_{\infty}\left[1+F_{c}^{2.867}\left(\frac{\left(2 \pi f \delta^{*}\right)}{U_{\infty}}\right)^{2}\right]} \\
F_{c}=\frac{1}{2}+\frac{h_{w}}{h_{a w}}\left(\frac{1}{2}+r \frac{\gamma-1}{4} M^{2}\right)+0.22 r \frac{\gamma-1}{2} M^{2}
\end{array}
$$

where:

$$
r=0.896 ; \gamma=1.4 \text { for air } ; \frac{h_{w}}{h_{a w}}=1 \text { for adiabatic wall }
$$

\subsubsection{Goody Model (2004) [22]}

Goody constructed a model to address inconsistencies in a previous model developed by Chase and Howe [91, 92] with respect to numerous experimental measurements covering a vast range of Reynolds numbers based on momentum thickness, $R_{\theta}$. The model is directed at modifying Chase and Howe's efforts to correct for their underestimation of the spectrum at low frequencies; as well as to adjust their prediction of the high frequency response to better fit the experimental results. 


$$
\Phi_{p}(f)=\frac{(2 \pi) 3\left(\delta / U_{\infty}\right)^{3}\left((2 \pi f) \tau_{w}\right)^{2}}{\left[\left((2 \pi f) \delta / U_{\infty}\right)^{0.75}+0.5\right]^{3.7}+\left[\left(1.1 R_{T}^{-0.57}\right)\left((2 \pi f) \delta / U_{\infty}\right)\right]^{7}}
$$

where:

$$
R_{T}=\frac{u_{\tau}^{2} \delta}{U_{\infty} \nu}
$$

\subsubsection{Rackl and Weston Model (2005) [93]}

The later work of Rackl and Weston at NASA Langley saw modifications to Efimtsov's model in the form of correction factors to adjust the high-frequency roll-off response of the model and to account for the turbulent energy peak surrounding a Strouhal number of 0.6. These corrections were based on flight test data obtained from a Tu-144LL aircraft which was fitted with flush mounted sensors on various window blanks. Equations 6.5b and 6.5c display the correction factors determined by Rackl and Weston, which can be added to Efimtsov's Model to obtain the corrected spectrum in Equation 6.5a.

$$
\begin{gathered}
\Phi_{p}(f)[d B]=\Phi_{p_{(\text {efimtsov })}}(f)[d B]+\Phi_{A}(f)[d B]+\Phi_{B}(f)[d B] \\
\Phi_{A}[d B]=2.5 e^{-\left[\ln \left(\frac{2 \pi f \delta^{*}}{U_{\infty}}\right)-\ln (0.6)\right]^{2}} \\
\Phi_{B}[d B]=0.25\left[\tanh \left(\log _{10}\left(\frac{f}{1000}\right)\right)+1\right](M-1.65) \log _{10}(f)
\end{gathered}
$$

Note that the correction factors, $\Phi_{A}(f)$ and $\Phi_{B}(f)$, are used to modify Efimtsov's model once it is converted to the decibel scale relative to the reference pressure of $20 \mu P a$ using Equation 6.6.

$$
\Phi_{p}(f)[d B]=10 \log _{10}\left(\frac{\Phi_{p}(f)}{p_{\text {ref }}^{2}}\right) ; p_{\text {ref }}=20 \mu \mathrm{Pa}
$$




\subsubsection{Estimation of Boundary Layer and Flow Parameters}

All of the provided spectrum models require inputs of flow and boundary layer parameters for their computations. Ideally, all of these parameters must be measured explicitly in the physical flow being examined for proper comparisons. However, this preliminary study excluded the use of measurement devices, such as a hotwire probe, to completely characterize the boundary layer properties within the test section. Therefore, the relationships outlined in Equations 6.7 - 6.11, obtained from literature [76, 93], were used to estimate the necessary parameters for input to the models. These include Reynolds number based on boundary layer growth distance, $R e_{x}$; boundary layer thickness, $\delta$; boundary layer displacement thickness, $\delta^{*}$; skinfriction coefficient, $C_{f}$; and wall shear stress, $\tau_{w}$. An estimated boundary layer growth distance, $x$, of $2.88 m$ was utilized for the calculation of the Reynolds number and corresponds to the streamwise duct length from the beginning of the settling cham-

ber to the location of the sensors on the test panel. Table 6.1 also contains averaged values of the base flow properties, measured within the test section, which were used to compute some of the parameters in Equations 6.7-6.11 for all flow conditions.

$$
\begin{gathered}
R e=\frac{U_{\infty} x}{\nu}=\frac{\rho U_{\infty} x}{\mu} \\
\delta=0.37 x R e^{-0.2}\left(1+0.144 M^{2}\right)^{0.35} \\
\delta^{*}=\frac{\delta\left(1.3+0.43 M^{2}\right)}{10.4+0.5 M^{2}\left(1+2\left(10^{-8}\right) R e_{x}\right)^{1 / 3}} \\
C_{f}=0.37 x R e^{-0.2}\left(1+0.144 M^{2}\right)^{0.35}
\end{gathered}
$$




$$
\tau_{w}=q_{\infty} C_{f}=\frac{1}{2} \rho U_{\infty}^{2} C_{f}
$$

Table 6.1: Measured flow properties within the test section.

\begin{tabular}{ccc}
\hline Mach Number & Static Pressure, $P[k P a]$ & Total Temperature, $T_{o}[K]$ \\
\hline \hline 0.1 & 102.68 & 289.9 \\
0.2 & 102.67 & 288.6 \\
0.3 & 102.67 & 283.8 \\
0.4 & 102.67 & 282.4 \\
0.5 & 102.62 & 281.4 \\
0.6 & 102.48 & 279.4 \\
0.7 & 102.36 & 276.3 \\
0.8 & 102.22 & 274.8 \\
\hline
\end{tabular}

It is important to note that the static temperature within the flow is obtained from the measured total temperature using the isentropic relationship outlined in Equation 6.12 [76].

$$
T=T_{o}\left(1+\frac{\gamma-1}{2} M^{2}\right)^{-1}
$$

Also, the density and dynamic viscosity of the fluid are estimated using both the pressure and temperature measurements, along with the ideal gas relationships and Sutherland's law (Equation 5.4.

\subsection{Signal Processing Methods}

This section details the procedures used to compute the TBL surface pressure fluctuation spectrum from raw microphone data. 


\subsubsection{Sampling and Signal Conditioning}

The output of the microphone sensors were sampled by the DAQ system at a rate of $20 \mathrm{kHz}$ to resolve frequencies up to the Nyquist frequency of $10 \mathrm{kHz}$. This upper limit was selected based on the general representation of the spectrum in literature data, as well as the frequency up to which the spectrum results start to attenuate well below the average relative signal magnitude. A Low-Pass Filter (LPF) was implemented to attenuate frequencies above $10 \mathrm{kHz}$ in the signal. A High-Pass Filter (HPF) was also used to attenuate erroneous frequency content in the signal below $20 \mathrm{~Hz}$ (lower frequency limit of the microphone). Signals were acquired for 30 seconds to obtain statistical convergence on the variance of the fluctuating pressure signal.

\subsubsection{Power Spectral Density Estimation}

The PSD of the fluctuating pressure measurements can be estimated using a variety methods. A common method used is Welch's method of averaged periodograms [21]. This method consists of splitting up the time signal into, $L$, non-overlapping segments

of equal length, $M$, and averaging the computed periodgram of each segment. This estimator is asymptotically unbiased and a more consistent statistical estimator in terms of the variance of the PSD; as opposed to computing a single periodogram of the entire signal [21].

For a given segment, $l$, of the discrete time signal, $x[n]$, containing $M$ points, the periodogram is computed using Equation 6.13.

$$
\Psi_{l}[k]=\frac{1}{\hat{U} M}\left|\sum_{n=1}^{M}\left[x_{l}[n] \mathrm{w}[n] e^{-i 2 \pi(k-1) \frac{(n-1)}{M}}\right]\right|^{2} \quad ; \quad \text { for } \quad 1 \leq k \leq M
$$

The segment $x_{l}[n]$ is first multiplied by an appropriate window function $\mathrm{w}[n]$ of 
length $M$. A window function of $\mathrm{w}[n]=1$ represents a rectangular window, however other types of windows can be used to decrease the spectral leakage caused by the relatively high sidelobe heights of the window's spectral response at the expense of some resolution (wider main lobe width). A hamming window (Equation 6.14 was selected for this analysis which provides a good combination of both for spectrum estimations [21].

$$
\mathrm{w}[n]=0.54-0.46 \cos \left(2 \pi \frac{(n-1)}{(M-1)}\right) \quad ; \quad \text { for } \quad 1 \leq n \leq M
$$

Once the segment is properly windowed, the Discrete Fourier Transform (DFT) of the data is calculated using a Fast Fourier Transform (FFT) algorithm with length $M$. The magnitude squared of the DFT coefficients is then normalized by the length of the DFT, $M$, and the window energy factor, $\hat{U}$. The energy factor, shown in Equation 6.15, represents the energy beneath the window function spectrum and is required to properly normalize the PSD estimate:

$$
\hat{U}=\frac{1}{M} \sum_{n=1}^{M} \mathrm{w}[n]^{2}
$$

where a rectangular window would yield a value of unity.

Once the periodogram is calculated for all $L$ segments, computing the average yields the estimate of the PSD in terms of the DFT coefficients, $k$ :

$$
\widehat{\Phi_{p}}[k]=\frac{1}{L} \sum_{l=1}^{L} \Psi_{l}[k] \quad ; \quad \text { for } \quad 1 \leq l \leq L
$$

In order to obtain the single-sided PSD estimate in terms of cyclic frequency, $\widehat{\Phi_{p}}(f)$, the discrete $\mathrm{PSD}, \widehat{\Phi_{p}}[k]$, must be normalized by the frequency interval of interest; which is zero to the Nyquist frequency $\left[0 \leq f \leq\left(f_{s} / 2\right)\right]$ : 


$$
\widehat{\Phi_{p}}(f)=\widehat{\Phi_{p}}\left(\frac{(k-1) f_{s}}{M}\right)=\left(\frac{2}{f_{s}}\right) \widehat{\Phi_{p}}[k] \quad ; \quad \text { for } \quad 1 \leq k \leq \frac{M}{2}+1
$$

where the DFT coefficient indices are converted to units of frequency using $[f=$ $\left.(k-1) f_{s} / M\right]$ and the DFT coefficients are only used up to $[(M / 2)+1]$ for an even length $M$.

As a sanity check, integrating the area underneath the estimated spectrum, from $0 \leq f \leq\left(f_{s} / 2\right)$, should yield the variance of the input discrete time signal. This ensures that the spectrum is properly normalized so that the entire power of the signal is contained within the selected frequency interval.

\subsection{Chamber Background Noise Measurements}

Before any TBL surface pressure fluctuation measurements were conducted, the background noise levels within the test chamber were characterized. This was performed to ensure that the expected magnitudes of the measured pressure fluctuation spectra are sufficiently larger than the measured background noise levels (SNR $\geq 10 d B$ ); as per the listed criteria in Chapter 2.1. Free-field chamber noise measurements were conducted using a single microphone, fitted with the free-field pressure measurement grid cap. The sensor was placed perpendicular to the nozzle orifice at a horizontal distance of $1.2 \mathrm{~m}$. The signal is processed in a similar manner as for the surface pressure measurements. Once the spectrum is calculated, it can be integrated to determine the overall mean-square fluctuating pressure level. Representation of this value on the decibel scale $(d B$ rel. $20 \mu \mathrm{Pa})$ provides a direct estimation of the Overall Sound Pressure Level (OASPL) within the chamber due to background noise. This value can then be compared to the averaged expected total power of the TBL pressure 
fluctuation signal by averaging the values of the integrated spectra predicted by the literature models.

Figure 6.1 contains the plot showing this comparison for the entire test section velocity range. The plots indicate that chamber background noise levels are within acceptable limits for almost all test conditions. However, issues of possible noise contamination may be prevalent around Mach 0.1 due to the close levels of background noise in comparison with the expected TBL noise. For this reason, alternative signal processing methods were implemented for the TBL spectrum measurements to ensure that any noise contamination is minimized.

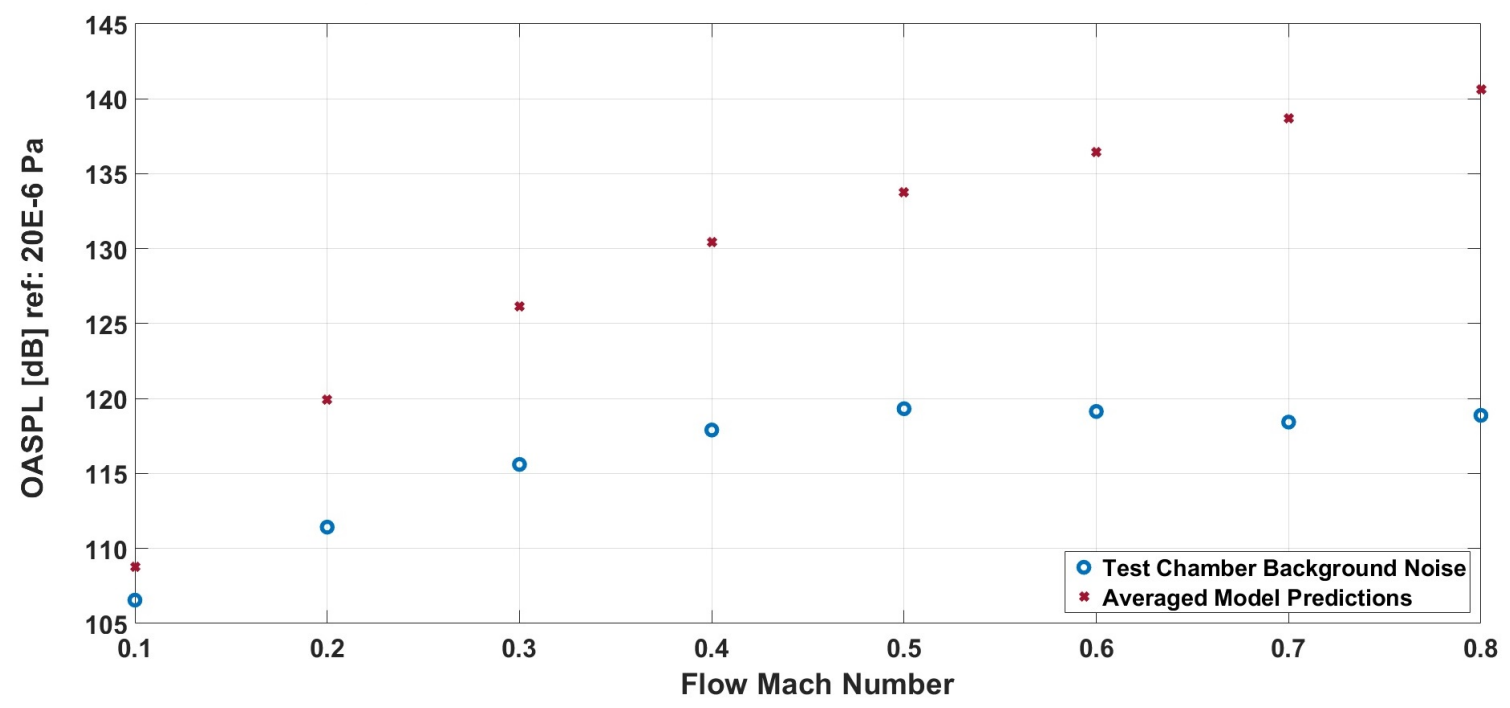

Figure 6.1: Comparison of overall chamber background noise levels with averaged model predictions of overall TBL signal power.

\subsection{Noise Cancellation Techniques}

Due to the potential for noise sources to contaminate the pressure fluctuation measurements (downstream propagation of control valve noise, air expansion out of tanks, panel vibrations, etc.) which are difficult to remove, a method for filtering out noise 
in the measurements outlined by Forest [33] was employed. This method is similar in nature to other spectral or temporal signal subtraction methods used to isolate the contributions due solely to the TBL. These techniques are commonly used in surface pressure fluctuation studies within wind tunnel environments in literature [9, 17, 94].

The technique exploits the fact that contributions from the turbulence and streamwise propagated noise to a wall pressure fluctuation signal are uncorrelated. This means that the single-point spectrum of the signal can be decomposed into its respective turbulent and acoustic noise components:

$$
\Phi_{p}=\Phi_{p_{t}}+\Phi_{p_{a}}
$$

Additionally, the calculated cross-spectral density between the main signal and one measured by a reference flush mounted sensor located at the same streamwise distance and spaced equidistantly from the tunnel centreline in the spanwise direction, $\Upsilon_{p r}$, can also be decomposed into its respective turbulent and acoustic noise components:

$$
\Upsilon_{p r}=\Upsilon_{p r_{t}}+\Upsilon_{p r_{a}}
$$

where the cross-spectrum is calculated in a similar manner as the single point spectrum (the periodogram becomes the normalized product of the DFT of the main signal and the complex conjugate of the DFT of the reference signal [21]).

If the additional reference sensor is placed at a sufficient spanwise distance away from the microphone of interest (at least one boundary layer thickness apart to ensure that the turbulent signals are not measuring contributions from the same coherent structures), the turbulent contribution component of the both signals becomes uncorrelated (assuming a statistically stationary and homogeneous TBL). Thus the turbulent component of the cross-spectrum between the two sensors is zero: 


$$
\Upsilon_{p r_{t}}=0
$$

Furthermore, both signals will measure the same acoustic noise component at the same streamwise position, which results in:

$$
\Phi_{p_{a}}=\Upsilon_{p r_{a}}
$$

Therefore, the contribution of the turbulent component in the main pressure fluctuation spectrum, $\Phi_{p_{t}}$, can be determined by subtracting the calculated crossspectrum between the two measured signals from the computed single-point spectrum measured by the main pressure fluctuation sensor at all frequencies of interest:

$$
\Phi_{p_{t}}=\Phi_{p}-\Upsilon_{p r}
$$

\subsection{Spectrum Results and Discussion}

Measurements of the single-point pressure fluctuation spectrum were conducted using two microphone sensors (\#4 \& \#5); one measuring the fluctuations at the point of interest and one for use as the reference signal for noise cancellation. The two microphones were flush mounted within the acrylic test panel which was rotated in a certain position so that the sensors were placed at the same streamwise distance from the nozzle orifice of $0.25 \mathrm{~m}$. The sensors have a spanwise separation distance of $2.54 \mathrm{~cm}$, which ensures they are separated larger than the expected boundary layer thickness. The data was segmented into 100 ensembles of equal length for estimation

of the power spectral density (refer to Chapter 6.2.2); based on convergence of the variance of the spectrum estimate. 
Figure 6.2 contains results of the measured spectrum for the entire range of governable test section Mach numbers within the HSAWT. The frequency axis is plotted on a logarithmic scale and the magnitude of the spectrum is represented on the decibel scale relative to $20 \mu \mathrm{Pa}$ (common representation method in literature).

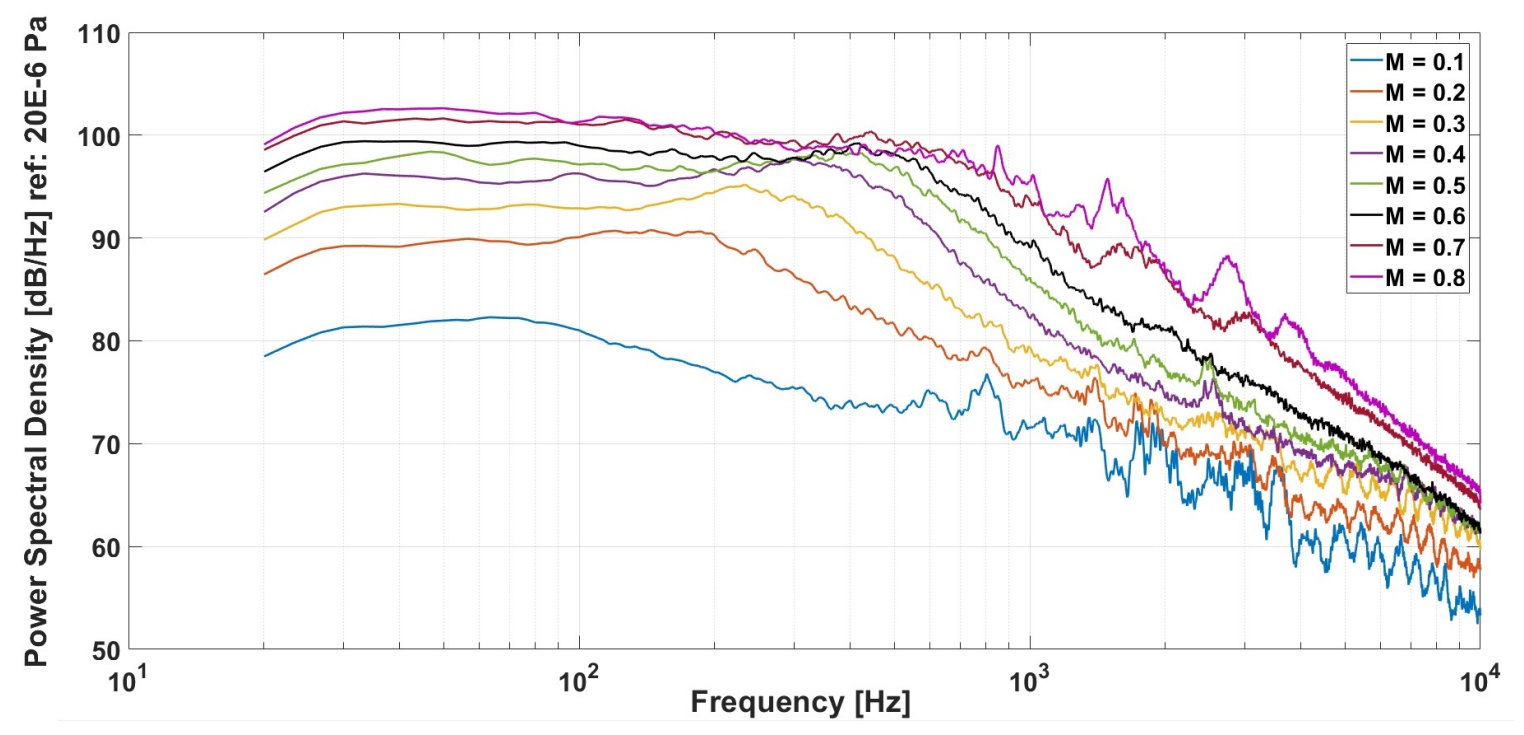

Figure 6.2: Comparison of measured single-point pressure fluctuation spectra for various test section Mach numbers.

The measured spectral behavior displays the expected trend of increasing power for higher flow speeds at all frequencies. The peaks of the spectra occur within the general area of 200-400 $\mathrm{Hz}$ and exhibit a main high frequency attenuation region of relatively constant slope. It is also interesting that the spectra between Mach 0.2 0.6 exhibit similar spectral peak and roll-off behavior as described by Rackl et al. [93] in their flight test measurements (slight 'hump' in spectral peak before attenuation). In general the spectra seem to display a lack of energy in the overlap/high frequency regions with too soon of a spectral peak roll-off as the speed is increased. The higher Mach number plots $(0.7-0.8)$ also seem to display a slight deficiency in energy around the peak region which may be indicative of the flow control system response. 
To obtain a better understanding of this behavior, the spectra in Figure 6.2 were normalized by various TBL properties to examine if the spectra collapse properly and display similar trends to the expected general behavior as discussed previously in Chapter 1.2.1 (refer to Figure 1.5). Figure 6.3 contains the normalized spectra with respect to the TBL parameters discussed in Chapter 1.2.1. The normalized spectra relative to the outer and mixed boundary layer variables are also compared with similar aggregate wind tunnel test data outlined by Miller [8] for speeds between Mach 0.1 and 0.5 .

Both the normalized spectra relative to outer and mixed TBL variables attempt to collapse the spectra in the low frequency to peak regions as expected, however the spectra do not completely collapse for all Mach numbers. The normalized spectra relative to the mixed TBL parameters push the spectral peak to the same nondimensional frequency value of roughly $2 \pi f \delta / u_{\tau}=50-60$. This is within the general expected value in literature according to Figure 1.5 and does roughly align with the normalized peak of the data obtained by Miller [8].

When looking at the normalized spectra relative to the inner TBL parameters, there is a lack of an expected collapse of the high frequency region, which illustrates the difficulty in charactering the expected high frequency response due to the sensitivity of this region to the development of the boundary layer and the sensors used to measure the phenomena (refer to Chapter 1.2.1).

There are various factors which could contribute to the behavior of the high frequency region of the results. One possibility is the use of empirical equations to estimate the inner TBL parameters (Chapter 6.1.6) is leading to a misrepresentation of the spectrum (ex: underestimated friction velocity), which can be solved through a more complete experimental investigation of the flow and boundary layer properties 


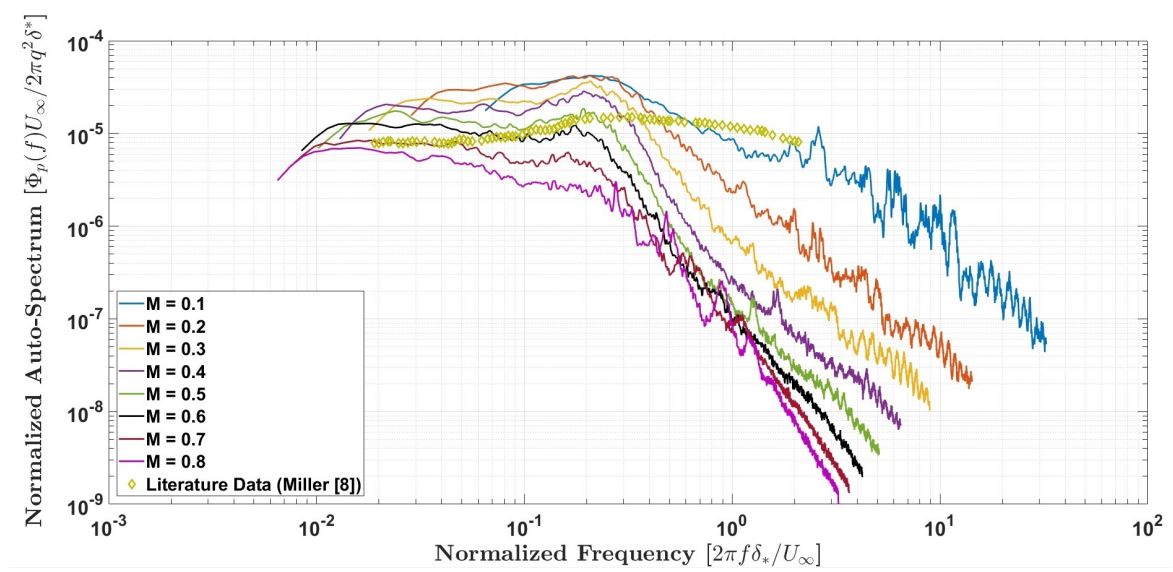

(a) Outer TBL variable normalization

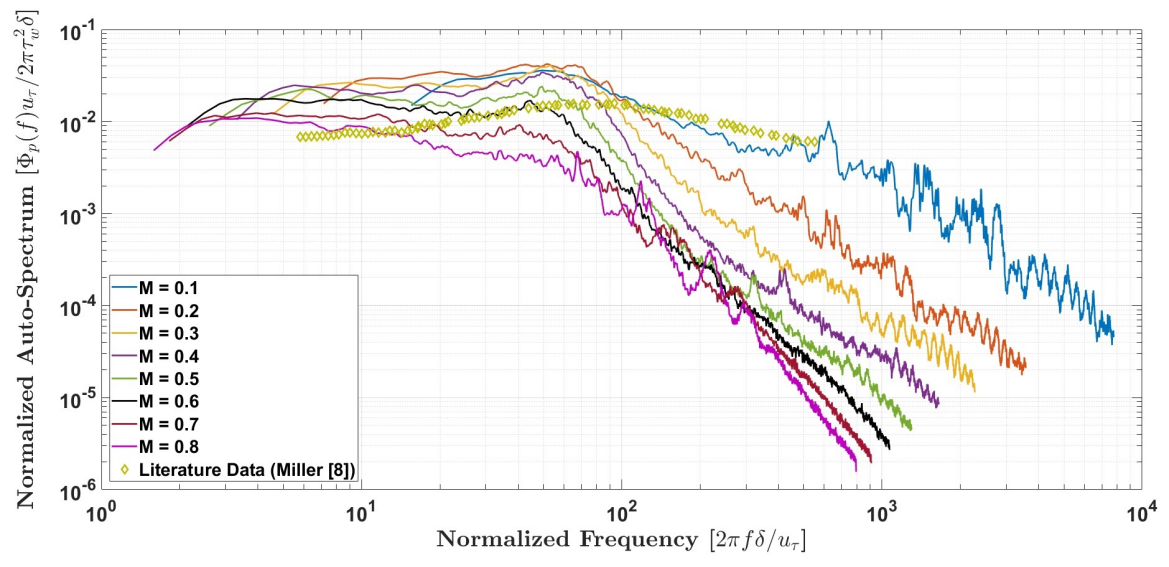

(b) Mixed TBL variable normalization

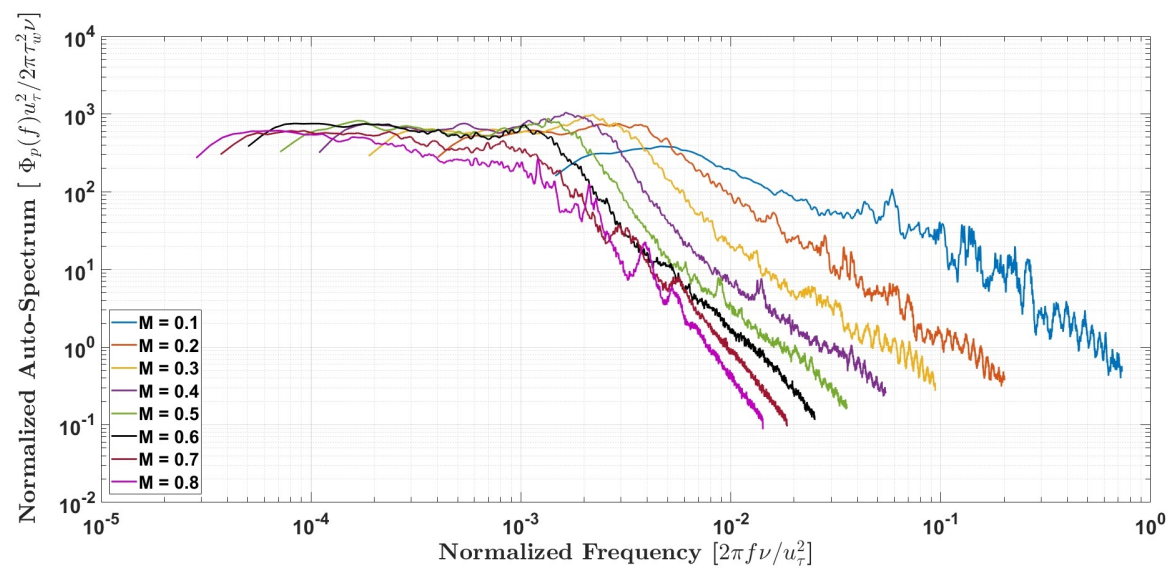

(c) Inner TBL variable normalization

Figure 6.3: Comparison of normalized pressure fluctuation spectra for various test section Mach numbers. 
which was not performed for this preliminary study. A second factor may be due to the sensor configuration, in which a finite sized sensor will at some point start to attenuate the signal at high frequencies [7, 31]. The ability of a transducer to accurately resolve the spectrum at high frequencies is dependent on the flow speed and the geometry of the sensor. This includes the size of the pin hole on the cap, the distance from the pinhole to the sensor diaphragm underneath, and the size of the diaphragm itself. Larger flow speeds and sensor geometric parameters will have a more difficult time in distinguishing the high frequency region of the spectrum [31, 95]. Schewe [31] proposed a non-dimensional sensor diameter, $d_{m i c}^{+}=d_{m i c} u_{\tau} / \nu$, which is used to quantify the ability of a sensor to obtain proper resolution of the high frequency spectrum. He argues that a sensor with a $d_{m i c}^{+}$value greater than 160 will introduce some error in the spectrum at high frequencies. Figure 6.4 contains calculated values of $d_{m i c}^{+}$at the various tunnel flow speeds. The plot shows that tunnel Mach numbers greater than 0.4 introduce sensor $d_{m i c}^{+}$values greater than 160 . This may explain the reason why the spectra for higher Mach numbers in Figure 6.2 seem to not vary at all within the high frequency region. Again, the calculation of this non-dimensional parameter requires accurate measurements of the friction velocity to ensure its validity. Moreover, an extensive study of the sensitivity of the high frequency spectrum to various sensor configurations may lead to a better understanding of the observed spectral behavior. This includes conducting measurements with various pinhole sizes and pinhole cap surface to sensor diaphragm distances. Another interesting approach proposed by Jaeger et. al [96] involves recessing the microphones with the exposed diaphragm directly underneath an acoustically transparent kevlar sheet, which may provide increased high frequency measurement sensitivity. 


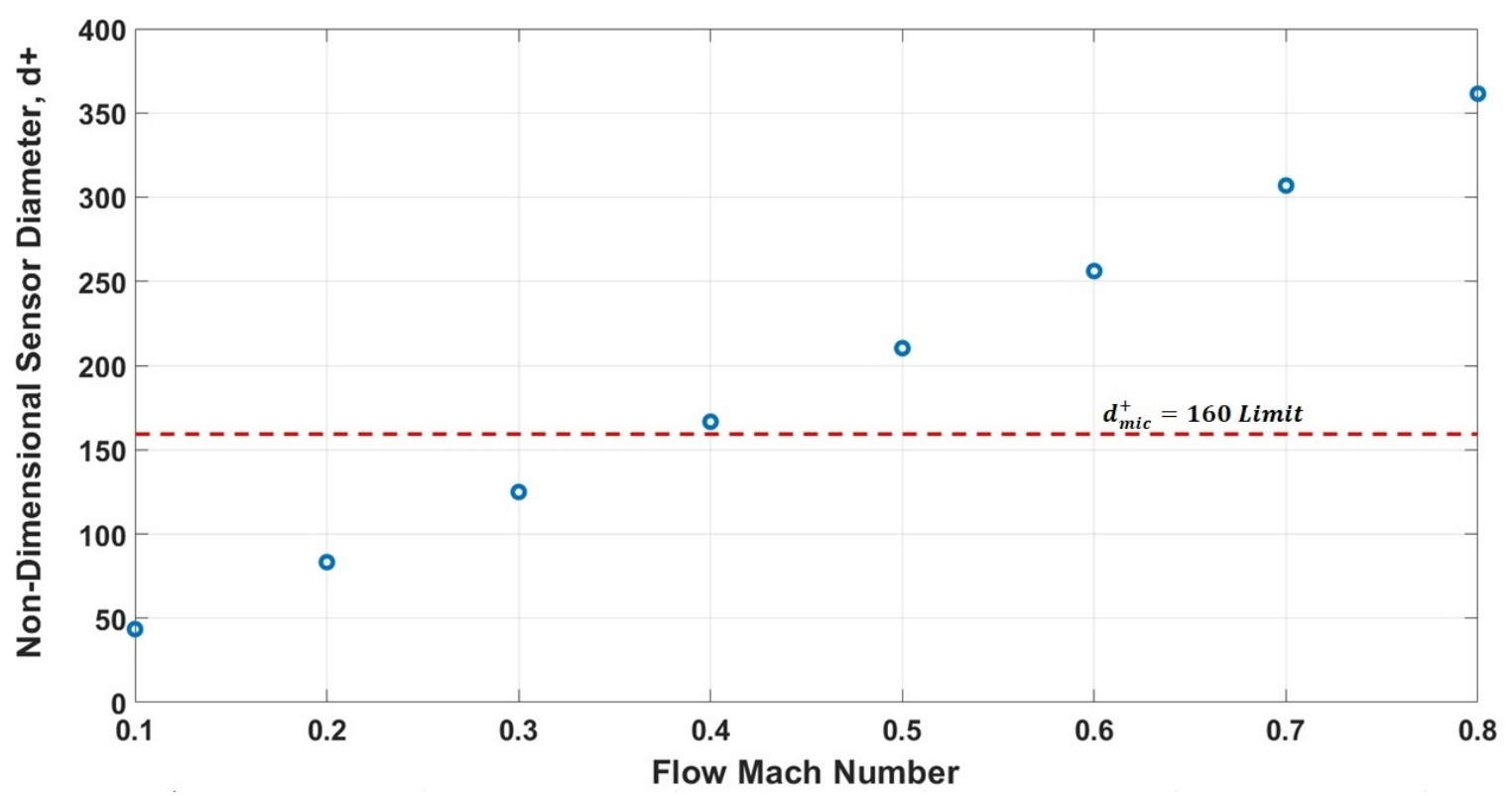

Figure 6.4: Estimated pressure fluctuation sensor non-dimensional diameter.

In addition to the stand-alone measurements presented, the individual spectra at each flow condition were also compared with the spectrum models presented in Chapter 6.1. As a verification for proper implementation, the models were plotted using flow and TBL parameters seen in typical flight test conditions for comparison with the implemented models through an analysis conducted by Rackel and Weston [93] for a flight speed of Mach 0.74. Figure 6.5(a) contains these plots using estimated TBL parameters for the specific flight conditions outlined by Rackel and Weston [93], while Figure 6.5(b) contains the same models for estimated TBL parameters which relate to the HSAWT set-up at the same Mach number. The major differences between the models is the shift in energy within the high frequency region, where the thinner boundary layer and higher fluid density conditions seen within the HSAWT test section contributes to a delayed attenuation roll-off and larger expected high frequency energy levels. 


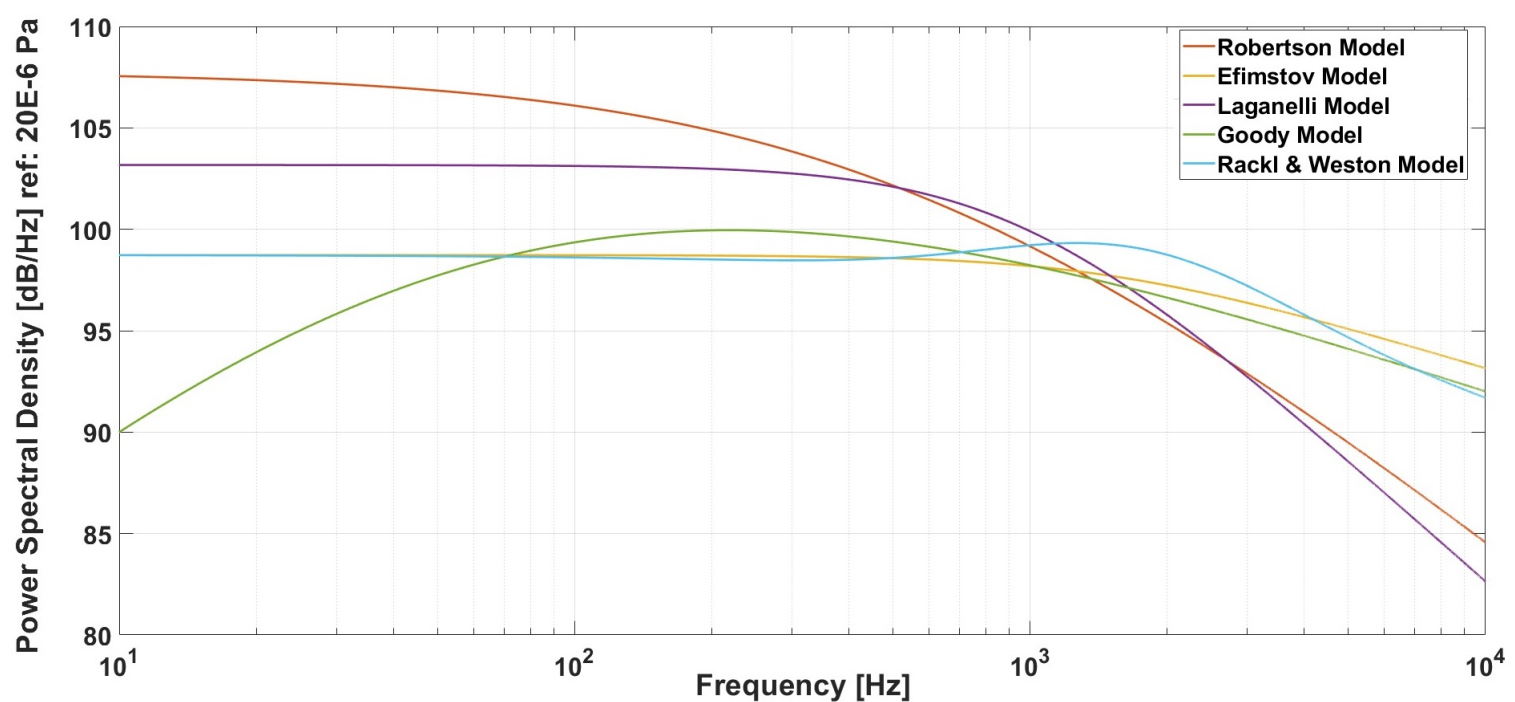

(a) Typical flight test TBL conditions

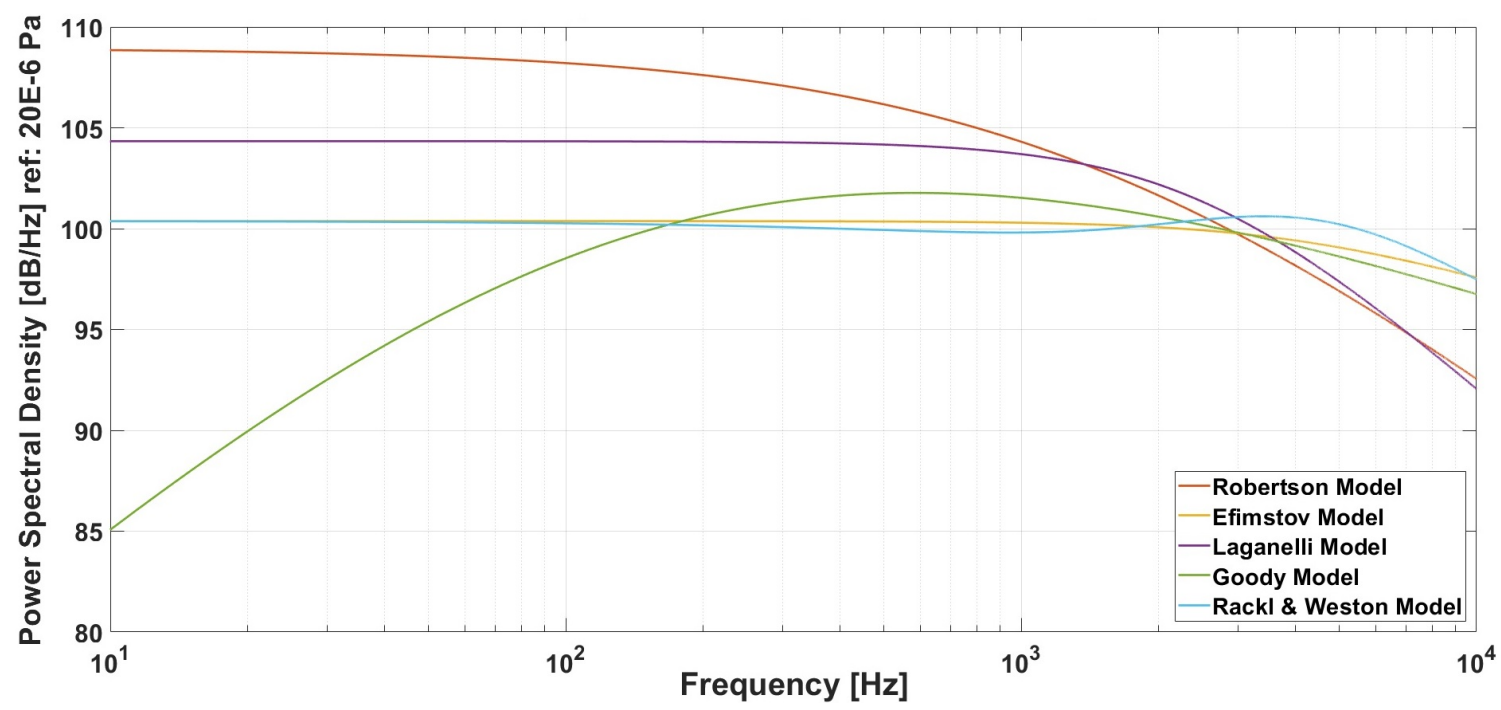

(b) HSAWT estimated TBL conditions

Figure 6.5: Comparison of pressure fluctuation spectrum literature model predictions - Mach 0.74 .

Figure 6.6 contains plots of the HSAWT low Mach number spectrum data comparisons with the model predictions outlined in Figure 6.5(b). In general, the plot for Mach 0.1 shows good agreement with Robertson's model within the low frequency range. The data also displays a similar high frequency attenuation slope as predicted by the models of Robertson and Laganelli. For Mach numbers greater than Mach 0.2, 


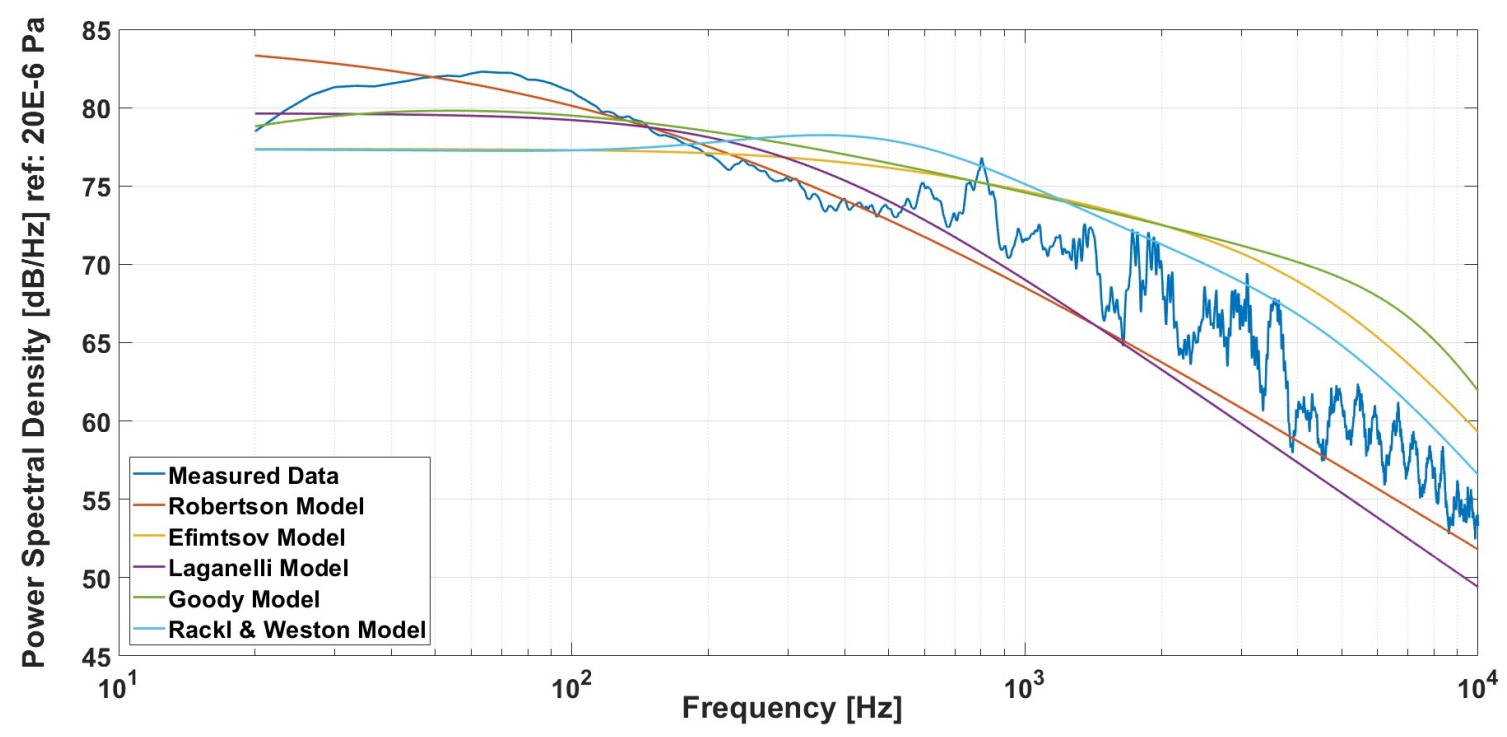

(a) Mach 0.1 data

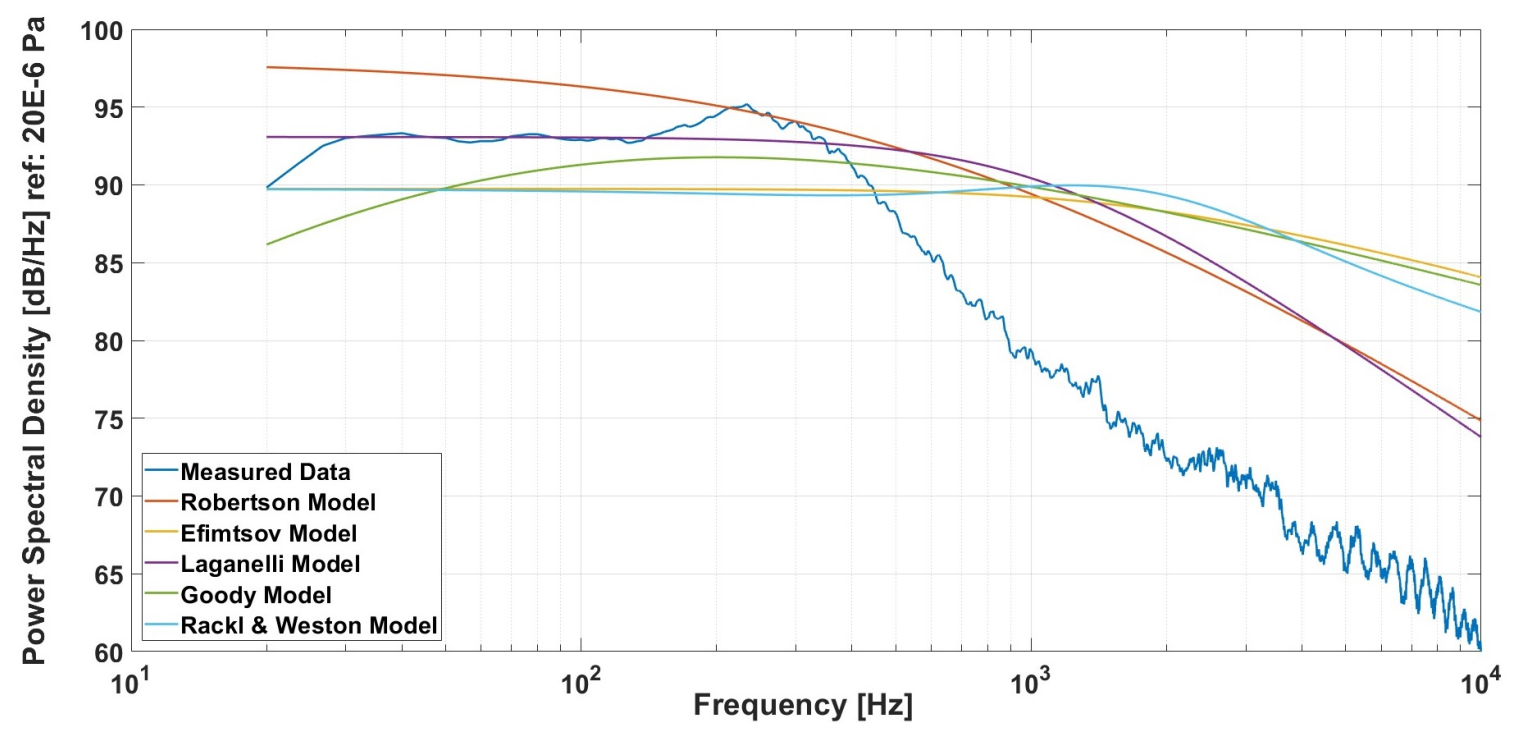

(b) Mach 0.3 data

Figure 6.6: Comparison of measured pressure fluctuation spectrum with literature models - Low Mach number range.

the models predict a large shift in the spectrum where it is not expected to start to roll-off until higher frequencies (consequence of wind tunnel testing conditions with thinner predicted boundary layers compared to aircraft with larger boundary layer growth distances). Although the spectrum coincides with Laganelli's model within 
the low frequency region at Mach 0.3, the measured spectrum exhibits a lack of energy past the spectral peak. The attenuation slope however seems to correspond with the two models.

For Mach numbers within the mid range of the HSAWT testing conditions (Figure 6.7), the spectrum starts to shift towards the lower levels predicted by Efimstov and Rackl \& Weston.

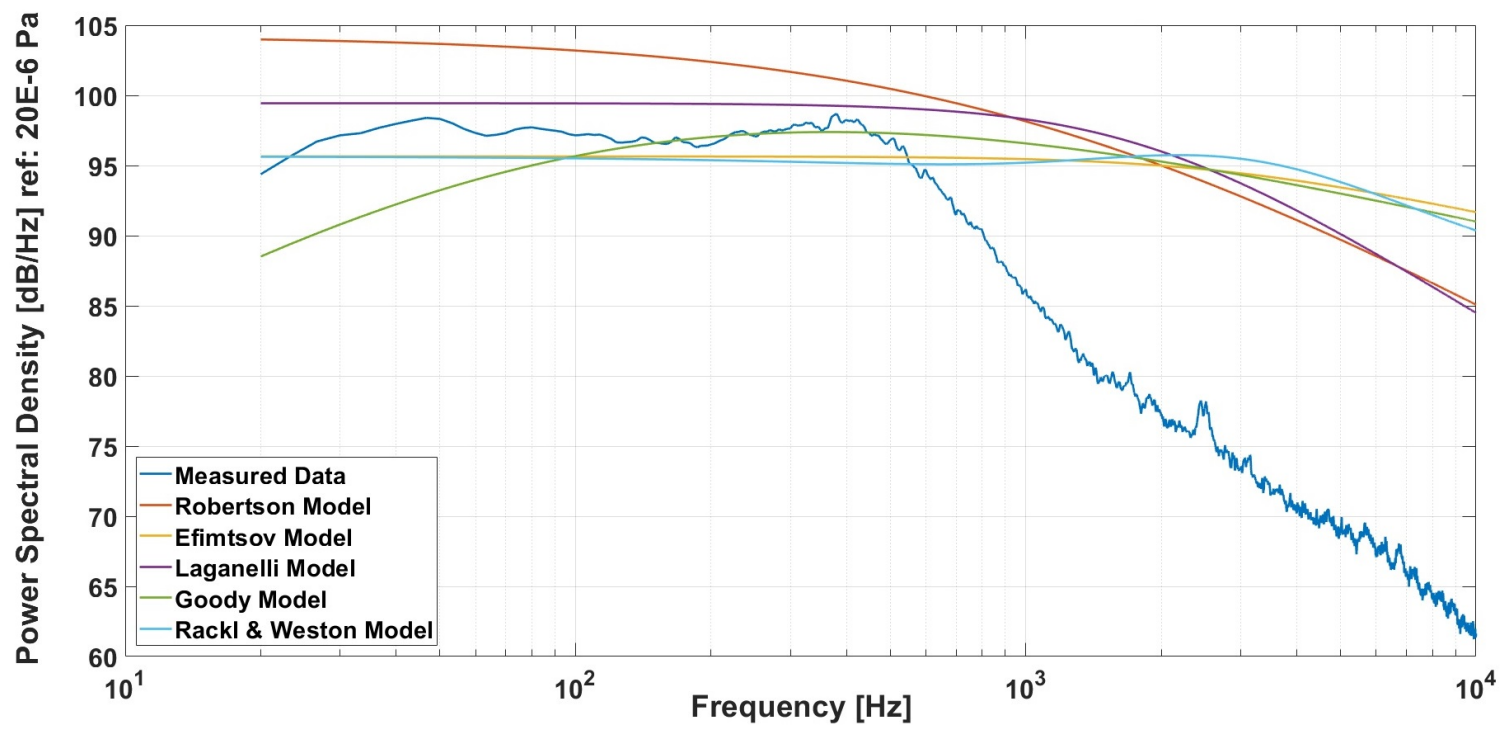

Figure 6.7: Comparison of measured pressure fluctuation spectrum with literature models - Mid Mach number range.

For higher speeds (Figure 6.8), these two models provide more accurate predictions of low frequency behavior compared to Robertson's model, however, the overlap and high frequency regions are still underrepresented in the measurements. It is important to note that the data at these high speeds are subject to larger influences from the flow control system as discussed in Chapter 5. The models themselves may also over estimate the high frequency spectrum for the current testing configuration since most of them are developed for spectral predictions under conditions with larger boundary layer thicknesses (more representative of flight conditions). 


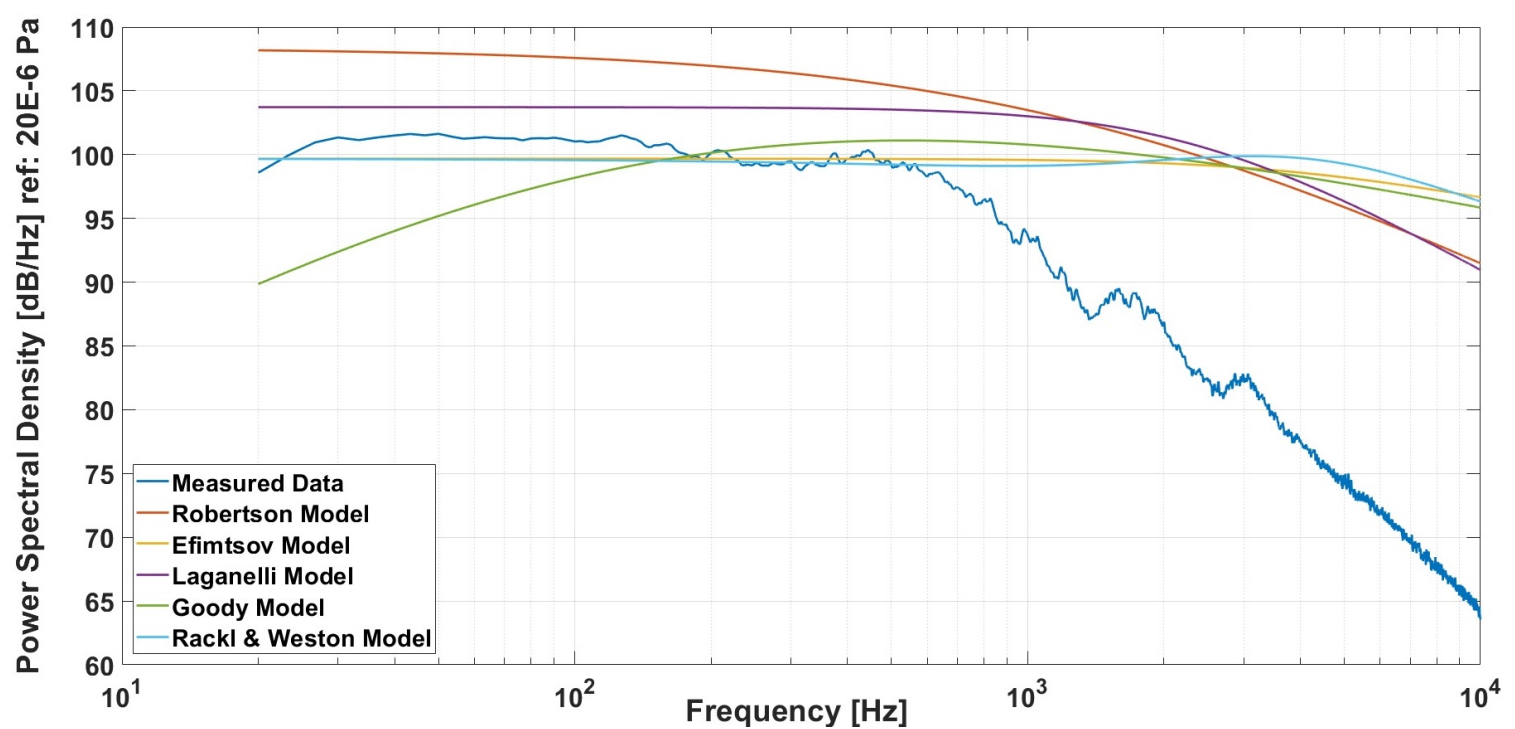

(a) Mach 0.7 data

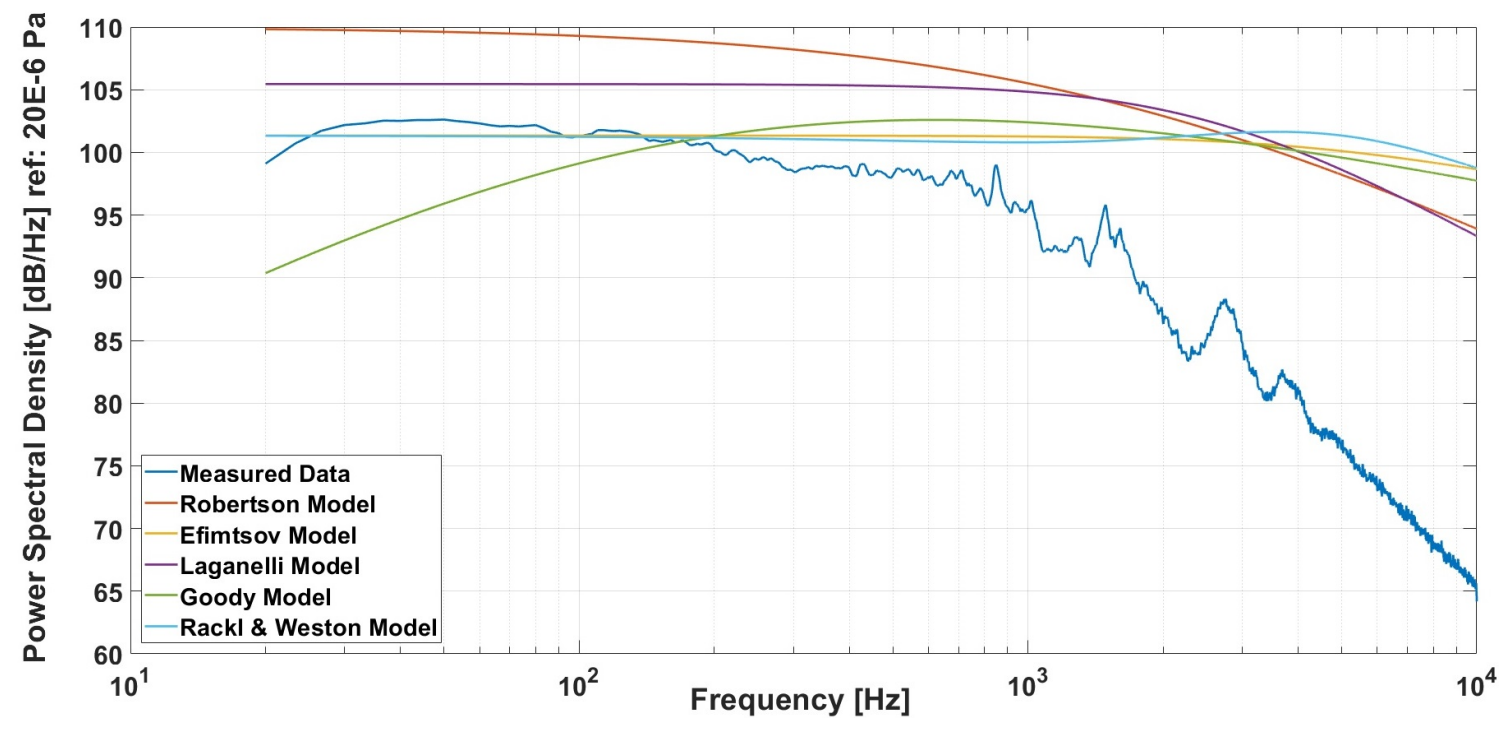

(b) Mach 0.8 data

Figure 6.8: Comparison of measured pressure fluctuation spectrum with literature models - High Mach number range.

For completion, the measured spectra were integrated across all frequencies of interest to obtain a single measure of the total TBL surface pressure fluctuation energy within the signal. These values were compared with the average of the integrated 
model spectrum predictions, as well as predictions from similar wind tunnel measurements in literature [7, 23], to compare relative differences in total spectral power. Figure 6.9 contains this data for all Mach numbers. Literature measurement data was only obtained for flow speeds at Mach 0.1 and 0.5 .

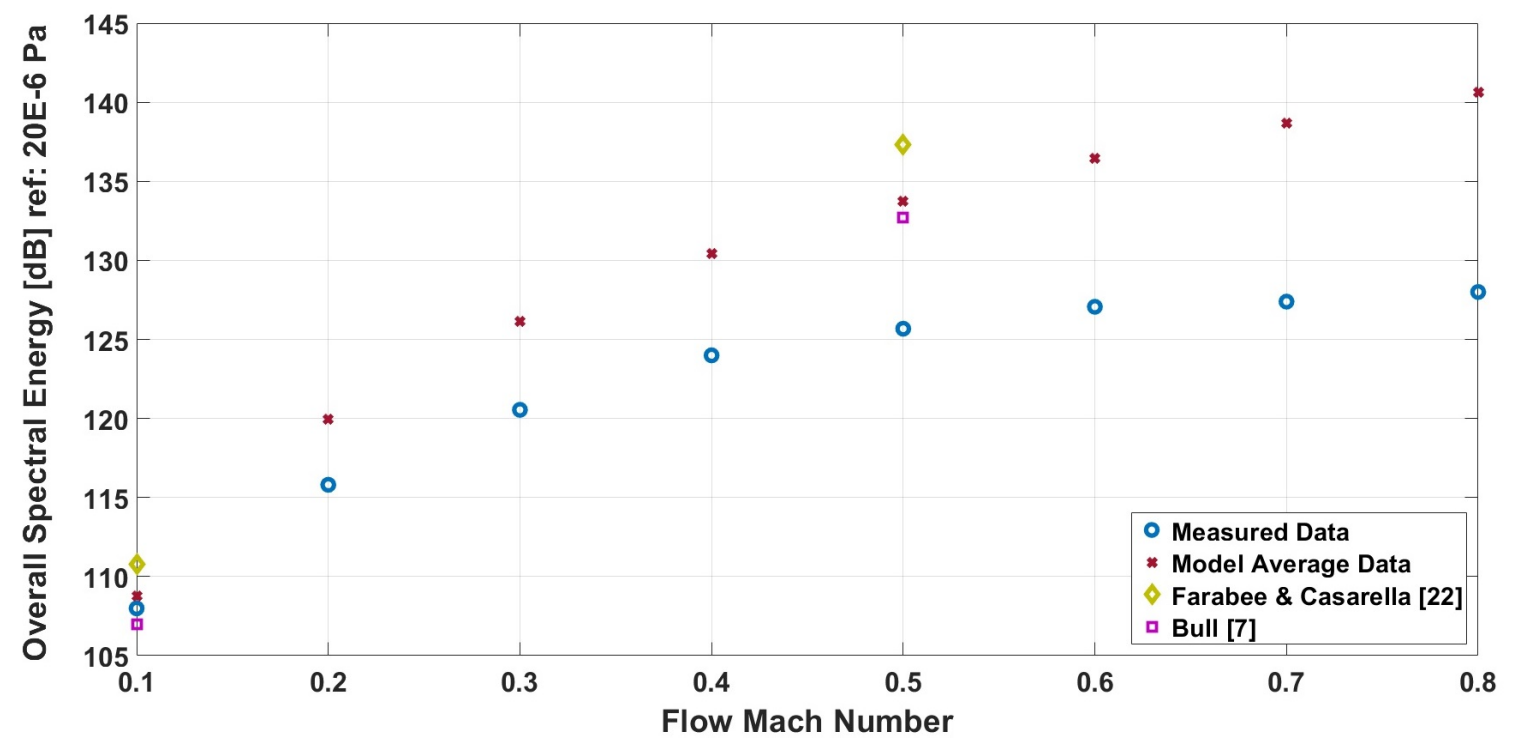

Figure 6.9: Overall TBL spectral energy level comparison.

The figure shows that the total power in the measured spectrum at Mach 0.1 is predicted by the models, and falls within reasonable comparative levels to measurements from Bull [7] and Farabee \& Casarella [23. The difference between the measurements and the model predictions however becomes increasingly larger as the speed is increased. This is also true for the spectral energy measured at Mach 0.5, which is lower than the measurements found in literature. This is indicative of the aforementioned conclusions in which the lack of energy in the high frequency region of the measured signal accounts for these differences in total energy. 


\section{Chapter 7}

\section{Conclusions and Future Recommendations}

\subsection{Conclusions}

This thesis provided a detailed overview of the design, fabrication, and testing of the new high-speed aeroacoustic blowdown wind tunnel facility at Carleton University. This wind tunnel is a unique facility in Canada, and one of the few aeroacoustic wind tunnels in the world capable of the wide range of subsonic test section velocities outlined. The purpose of the wind tunnel, among other potential aeroacoustic research directives, is to provide a test bed for turbulent boundary layer induced surface pressure fluctuation studies. This initiative was spawned from a need of increased capabilities in terms of expanding previously conducted research from low to higher speed environments.

The complete scope of work included the design of the anechoic chamber, the enclosed open-jet test section, the upstream wind tunnel components (including the nozzle and settling chamber), and the downstream test section exhaust components (including the test section jet collector, diffuser system, and required corner turning vane rack). The analytical design methods used for the major flow components were also validated with CFD analyses to ensure proper aerodynamic design. The 
components were fabricated and assembled within the facility and all necessary instrumentation and control system hardware was implemented to enable commissioning of the facility.

The facility commissioning and initial surface pressure fluctuation studies conducted provides the necessary ground work for future research and improvements. The wind tunnel was able to obtain governed test section flow speeds up to Mach 0.6 with relatively good control. Test sections speeds up to the maximum target of Mach 0.8 were found to have reduced controllability for larger run times. Measurements of the background noise levels within the anechoic chamber were determined to be sufficiently lower than the signal magnitude of the measured surface pressure fluctuation measurements at all test section speeds greater than Mach 0.2. Additional signal processing methods were used to help eliminate noise contamination in the measurements at the low speed range.

Preliminary measurements of the surface pressure fluctuation spectra indicated the general expected behavior of increased TBL pressure fluctuation energy for increasing test section flow speeds. The behavior of the spectra was generally in good agreement with the expected behavior and predicted spectra from the empirical models within the low frequency range. More notably, the spectra followed in agreement with the elevated predictions of Robertson's and Laganelli's models for lower relative test section speeds (Mach 0.1 to 0.4 ). Efimtsov's and Rackl/Weston's models were found to be more accurate in predicting the low frequency spectrum levels for higher Mach numbers (0.6 to 0.8). The spectra in general, however, displayed a lack of spectral energy within the mid-to-high frequency range for flow speeds greater than Mach 0.2 compared with expected behavior in literature. This discrepancy could be attributed to various factors such as the lack of Mach number control for the high 
speed range, attenuation of the spectrum due to the sensor configuration, underdeveloped TBL in the test section, and/or over estimated high frequency energy levels by the models for thinner TBL conditions. In order to completely isolate for the cause of this, a full study on the test section meanstream flow and TBL characteristics is suggested for future work, along with a full sensitivity study on the high frequency component of the spectrum to various flush mounted sensor configurations.

\subsection{Future Recommendations}

There are several recommendations for future work involving possible facility improvements and next steps in light of the conducted studies and experimental results. These fall into the main categories of control system improvements and detailed flow characterization studies.

\subsubsection{Control System and Instrumentation Improvements}

There are some possible methods of improving the control system response at high Mach numbers if optimization of the control and sampling parameters is not able to completely produce a steadier response. One method is to increase the reservoir tank storage pressure limits until the maximum possible is achieved without compromising any safety regulations. This would enable larger run times before the control valve opening position cannot keep up with the target opening rate. Another possibility is to reduce the required mass flow rate through the tunnel. This can be done by reducing system pressure losses with slight modifications to the tunnel components (ex. a second flow conditioning screen may not be needed if acceptable turbulence metrics are achieved in the test section). Another more robust method is to implement closed test section walls around the test panel using acoustically transparent material 
such as Kevlar (this type of set-up has been studied and implemented in various aeroacoustic facilities such as the one at Virginia Polytechnic Institute and State University [33, 46] which is shown in Figure 7.1). Implementing a closed wall test section would lower pressure losses in the test section and would also lower the test section static pressure significantly to be able to achieve the same governed flow speeds at a lower required test section total pressure and mass flow rate.

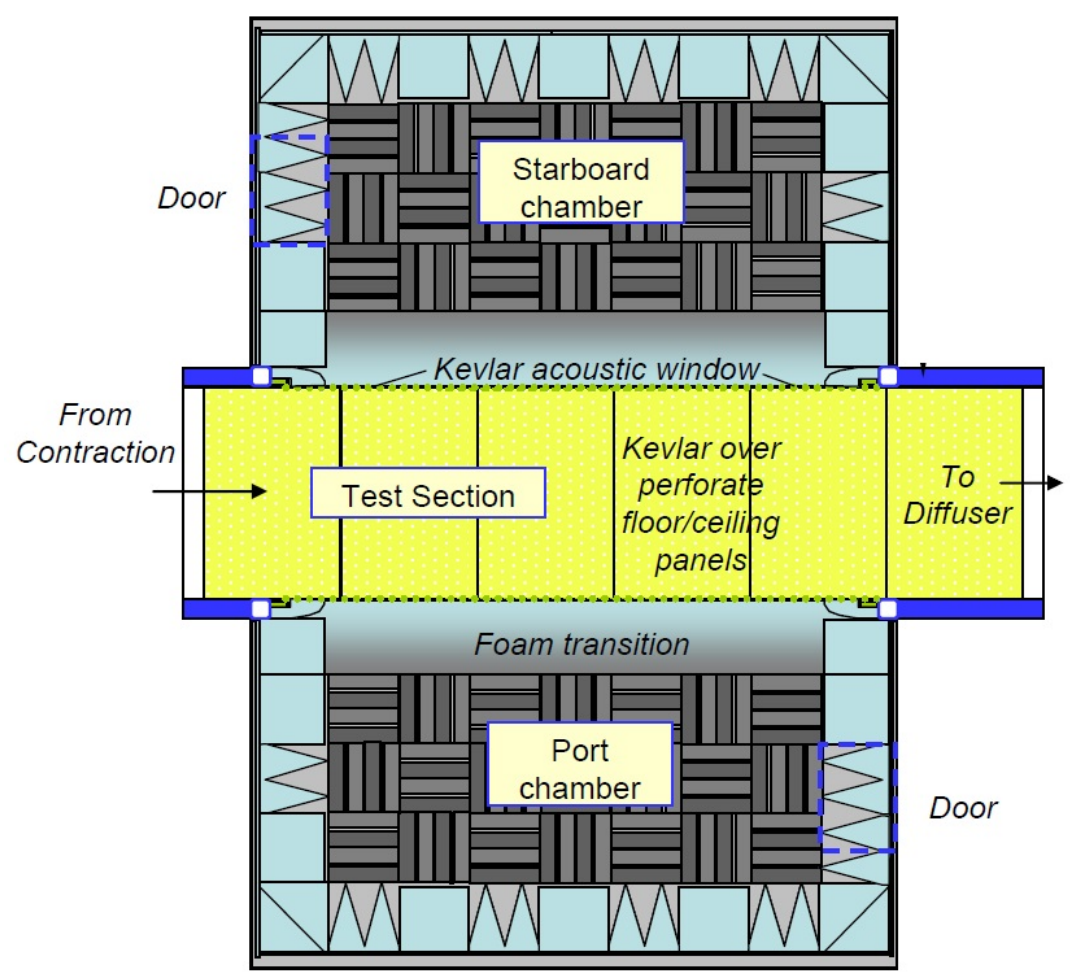

Figure 7.1: Virginia Polytechnic Institute and State University aeroacoustic wind tunnel with closed Kevlar test section walls (adapted from [46]).

In addition to these suggestions, the use of more accurate transducers would improve the uncertainty in the control system response for lower Mach numbers. 


\subsubsection{Recommended Flow Characterization Studies}

It is recommended that future work entail the complete characterization of the flow field within the test section to properly ensure the validity of the preliminary results of the measured surface pressure fluctuation spectra. This includes the following recommended studies:

1. A full examination of the test section jet profile to validate the CFD results and to ensure that the pressure fluctuation sensors are located within the potential core of the jet;

2. Measurements of the streamwise static pressure profile within the test section to validate zero mean streamwise pressure gradient conditions over the panel;

3. Full characterization of the boundary layer at the measurement location, including the mean velocity profile and integral boundary layer parameters (friction velocity, boundary layer thickness, and displacement thickness);

4. Measurements of test section flow non-uniformity and turbulence intensity;

5. Measurements of the pressure fluctuation spectra using alternate sensor configurations (ex. flush diaphragm recessed underneath kevlar cover [17]) and diaphragm cap geometries (ex. smaller pin-hole with less space between the cap and sensor diaphragm) to examine their sensitivity on the high frequency region of the spectrum;

6. Analysis of potential signal contamination and degraded high frequency resolution of the spectrum measurements due to vibrations within the test panel using a mounted accelerometer. 


\section{Bibliography}

[1] J. Wilby, "Aircraft interior noise," Journal of Sound and Vibration, vol. 190, pp. 545-564, 1996.

[2] E. Richards and D. Mead, Noise and Acoustic Fatigue in Aeronautics. New York: John Wiley and Sons, 1968.

[3] F. Fahy and J. Walker, Advanced Applications in Acoustics: Noise and Vibration. London: Spon Press, 2004.

[4] N. Hu, H. Buchholz, and M. Herr, "Contributions of different aeroacoustic sources to aircraft cabin noise," in 19th AIAA/CEAS Aeroacoustics Conference, Berlin, Germany, pp. 1-10, May 2013.

[5] D. Bishop, "Cruise flight noise levels in a turbojet transport airplane," Noise Control, vol. 12, pp. 18-25, 2008.

[6] R. Hayden, B. Murray, and M. Theobald, "A study of interior noise levels, noise sources and transmission paths in light aircraft," NASA Langley Research Center, vol. Contractor Report CR-172152, pp. 1-198, 1983.

[7] M. Bull, "Wall-pressure fluctuations beneath turbulent boundary layers: Some reflections on forty years of research," Journal of Sound and Vibration, vol. 190, pp. 299-315, 1996. 
[8] T. Miller, Turbulent Boundary Layer Models for Acoustic Analysis. PhD Dissertation: Wichita State University, 2002.

[9] T. Miller, J. Gallman, and M. Moeller, "Review of turbulent boundary-layer models for acoustic analysis," Journal of Aircraft, vol. 49, pp. 1739-1754, 2012.

[10] R. Kraichnan, "Pressure fluctuations in turbulent flow over a flat plate," Journal of the Acoustical Society of America, vol. 28, pp. 378-390, 1956.

[11] J. D'Angelo, Attenuation of Turbulent Boundary Layer Induced Interior Noise Using Smart Foam Elements. PhD Dissertation: Virginia Polytechnic Institute and State University, 2004.

[12] V. Mellert, I. Baumann, N. Freese, and R. Weber, "Impact of sound and vibration on health, travel comfort and performance of flight attendants and pilots," Aerospace Science and Technology, vol. 7, pp. 37-42, 1961.

[13] M. Alves-Pereira and C. Branco, "Vibroacoustic disease: Biological effects of infrasound and low-frequency noise explained by mechanotransduction cellular signalling," Progress in Biophysics and Molecular Biology, vol. 93, pp. 256-279, 2007.

[14] D. Bies, A Review of Flight and Wind Tunnel Measurements of Boundary Layer Pressure Fluctuations and Induced Structural Response. Technical Report CR626: NASA Langley Research Center, 1996.

[15] Y. Hwang, W. Bonness, and S. Hambric, "Comparison of semi-empirical models for turbulent boundary layer wall pressure spectra," Journal of Sound and Vibration, vol. 319, pp. 199-217, 2009. 
[16] J. V. Blitterswyk and J. Rocha, "An experimental study of the wall-pressure fluctuations beneath low reynolds number turbulent boundary layers," Journal of the Acoustical Society of America, vol. 141, pp. 1257-1268, 2017.

[17] J. V. Blitterswyk, Experimental Characterization of Turbulent Motions using Wall-Pressure Measurements in Low Reynolds Number Turbulent Boundary Layers. MASc Thesis: Carleton University, 2016.

[18] H. Schlichting, Boundary Layer Theory. New York: McGraw-Hill, 1979.

[19] M. Perlin, D. Dowling, and S. Ceccio, "Freeman scholar review: Passive and active skin-friction drag reduction in turbulent boundary layers," ASME Journal of Fluids Engineering, vol. 138, pp. 1-16, 2016.

[20] D. Juve, M. Berton, and E. Salze, "Spectral properties of wall-pressure fluctuations and their estimation from computational fluid dynamics," Flow Induced Noise and Vibration Issues and Aspects, vol. Flinovia, pp. 27-46, 2015.

[21] J. Karl, An Introduction to Digital Signal Processing. San Diego: Academic Press Inc., 1989.

[22] M. Goody, "Empirical spectral model of surface pressure fluctuations," American Institute of Aeronautics and Astronautics, vol. 42, pp. 1788-1794, 2004.

[23] T. Farabee and M. Casarella, "Spectral features of wall pressure fluctuations beneath turbulent boundary layers," Physics of Fluids, vol. 3, pp. 2410-2420, 1991.

[24] J. F. Williams, "Surface-pressure fluctuations induced by boundary-layer flow at finite mach number," Journal of Fluid Mechanics, vol. 22, pp. 507-519, 1965. 
[25] R. Panton and J. Linebarger, "Wall pressure spectra calculations for equilibrium boundary layers," Journal of Fluid Mechanics, vol. 65, pp. 261-287, 1974.

[26] D. Palumbo, "Determining correlation and coherence lengths in turbulent boundary layer flight data," Journal of Sound and Vibration, vol. 331, pp. 3721-3737, 2012.

[27] W. Blake, "Turbulent boundary-layer wall-pressure fluctuations on smooth and rough walls," Journal of Fluid Mechanics, vol. 44, pp. 637-660, 1970.

[28] E. Salze and et al., "An experimental characterisation of wall pressure wavevector-frequency spectra in the presence of pressure gradients," in 20th AIAA/CEAS Aeroacoustics Conference, Atlanta, GA, pp. 1-26, June 2014.

[29] P. Bradshaw, "'inactive' motion and pressure fluctuations in turbulent boundary layers," Journal of Fluid Mechanics, vol. 30, pp. 241-258, 1967.

[30] S. Gravante, "Characterization of the pressure fluctuations under a fully developed turbulent boundary layer," American Institute of Aeronautics and Astronautics, vol. 36, pp. 1808-1816, 1998.

[31] J. Murphy, D. Bies, W. Speaker, and P. Franken, "On the structure and resolution of wall-pressure fluctuations associated with turbulent boundary-layer flow," Journal of Fluid Mechanics, vol. 134, pp. 311-328, 1983.

[32] J. Hurault and et al., "Aeroacoustic wind tunnel experiment for serration design optimisation and its application to a wind turbine rotor," in 6th International Meeting on Wind Turbine Noise, Glasgow, Scotland, pp. 1-16, April 2015.

[33] J. Forest, The Wall Pressure Fluctuation Spectrum of High Reynolds Number 
Rough-Wall Turbulent Boundary Layers. MSc Thesis: Virginia Polytechnic Institute and State University, 2012.

[34] A. Eckel and I. Ver, "Design and construction considerations for automotive and automotive component acoustic test facilities," in 2002 International Congress and Exposition on Noise Control Engineering, Dearborn, MI, pp. 1-8, August 2002.

[35] T. Chong, P. Joseph, and P. Davies, "Design and performance of an open jet wind tunnel for aero-acoustic measurement," Journal of Applied Acoustics, vol. 70, pp. 605-614, 2009.

[36] T. Chong, P. Joseph, and P. Davies, Design and Characterisation of a Quiet, Low Turbulence Open Jet Blow Down Wind Tunnel in ISVR. University of Southampton: ISVR Technical Report No 322, 2008.

[37] K. Pascioni, R. Reger, A. Edstrand, and L. Cattafesta, "Characterization of an aeroacoustic wind tunnel facility," in Inter-Noise 2014, Melbourne, Australia, pp. 1-10, November 2014.

[38] D. Leclercq, C. Doolan, and J. Reichl, "Development and validation of a smallscale anechoic wind tunnel," in 14th International Congress on Sound and Vibration, Cairns, Australia, pp. 1-8, July 2007.

[39] V. Ciobaca, S. Melber-Wilkending, and M. Pott-Pollenske, "A cfd process chain for simulating open wind tunnel test sections," in KATnet II Conference on Key Aerodynamic Technologies, Germany, pp. 1-10, May 2009.

[40] R. Putzu, D. Greco, D. Craquelin, and F. Crisinel, "Design and construction of a silent wind tunnel for aeroacoustic research," in 4th CEAS Air and Space Conference, Linkoping, Sweden, pp. 1-7, September 2013. 
[41] D. Chow, Design and Characterization of the UTIAS Anechoic Wind Tunnel. MASc Thesis: University of Toronto, 2016.

[42] P. Soderman and L. Olson, Large-Scale Aeroacoustic Research Feasibility and Conceptual Design of Test-Section Inserts for the Ames 80-by 120-Foot Wind Tunel. NASA Ames Research Center: NASA Technical Paper 3020, 1990.

[43] T. Mueller and et al., "The design of a subsonic low-noise, low-turbulence wind tunnel for acoustic measurements," (92-3883), AIAA Technical Publication, 1992.

[44] J. Mathew, Design, Fabrication, and Characterization of An Anechoic Wind Tunnel Facility. PhD Dissertation: University of Florida, 2006.

[45] E. Sarradj, C. Fritzsche, T. Geyer, and J. Giesler, "Acoustic and aerodynamic design and characterization of a small-scale aeroacoustic wind tunnel," Journal of Applied Acoustics, vol. 70, pp. 1073-1080, 2009.

[46] M. Remillieux and et al., "Calibration and demonstration of the new virginia tech anechoic wind tunnel," in 14th AIAA/CEAS Aeroacoustics Conference, Vancouver, pp. 1-10, May 2008.

[47] M. Jeffries, The Commissioning and Enhancement of a Blow Down Wind Tunnel. MEng Thesis: Carleton University, 1994.

[48] M. Kibsey, Influence of Mach Number on Profile Loss of Axial-Flow Turbine Blades. MASc Thesis: Carleton University, 2015.

[49] I. Idelchik, Handbook of Hydraulic Resistance. New York: Begell House, 1996.

[50] S. Hall, Investigation of the Effects of Compressibility on Profile Losses in Axial Turbine Cascades. MASc Thesis: Carleton University, 2012. 
[51] Hosecraft USA, Chicago, IL, Flexible Hose Product Guide, 2017.

[52] R. Mehta and P. Bradshaw, "Design rules for small low speed wind tunnels," Royal Aeronautical Society, vol. 7, pp. 443-449, 1961.

[53] A. Pope and J. Harper, Low-Speed Wind Tunnel Testing. New York: John Wiley and Sons, Inc., 1966.

[54] J. Scheiman, Considerations for the Installation of Honeycomb and Screens to Reduce Wind-Tunnel Turbulence. NASA Langley Research Center: NASA Technical Memorandum 81868, 1981.

[55] I. Beckwith, Comments on Settling Chamber Design for Quiet, Blowdown Wind Tunnels. NASA Langley Research Center: NASA Technical Memorandum 81948, 1981.

[56] R. Loehrke and H. Nagib, "Control of free-stream turbulence by means of honeycombs: A balance between suppression and generation," Journal of Fluids Engineering, vol. 98, pp. 342-351, 1976.

[57] R. Pinker and M. Herbert, "Pressure loss associated with compressible flow through square-mesh wire gauzes," Journal of Mechanical Engineering Science, vol. 9, pp. 11-23, 1967.

[58] G. Schubauer, W. Spangenberg, and P. Klebanoff, Aerodynamic Characteristics of Damping Screens. National Advisory Committee for Aeronautics: NACA Technical Note 2001, 1958.

[59] K. Wieghardt, "On the resistance of screens," Aeronautical Quarterly, vol. 4, pp. 186-192, 1953. 
[60] C. Su and C. Huang, "Experimental studies of flow through single gauzes," International Journal of Heat and Fluid Flow, vol. 12, pp. 273-278, 1991.

[61] T. Morel, "Design of two-dimensional wind tunnel contractions," Journal of Fluids Engineering, vol. 76, pp. 371-377, 1977.

[62] J. Sargison, G. Walker, and R. Rossi, "Design and calibration of a wind tunnel with a two dimensional contraction," in 15th Australasian Fluid Mechanics Conference, Sydney, Australia, pp. 1-4, December 2004.

[63] S. Ramaseshan and M. Ramaswamy, "A rational method to choose optimum design for two-dimensional contractions," Journal of Fluids Engineering, vol. 124, pp. 544-546, 2002.

[64] B. Stratford, "The prediction of separation of the turbulent boundary layer," Journal of Fluid Mechanics, vol. 5, pp. 1-16, 1959.

[65] 2011 ASHRAE Handbook - HVAC Applications, ch. 48: Noise and Vibration Control. 2011.

[66] R. Wells, "Acoustical plenum chambers," Noise Control, Acoustical Society of America, vol. 4, pp. 217-223, 1958.

[67] A. Cummings, "The attenuation of lined plenum chambers in ducts: Theoretical models," Journal of Sound and Vibration, vol. 61, pp. 347-373, 1978.

[68] T. Schultz, Acoustical Uses for Perforated Metals: Principles and Applications. Boca Raton, FL: Industrial Perforators Association Inc., 1986.

[69] H. Hubbard and J. Manning, Aeroacoustic Research Facilities at NASA Langley Research Center - Description and Operational Characteristics. NASA Langley Research Center: NASA Technical Memorandum 84585, 1983. 
[70] G. Abramovich, The Theory of Turbulent Jets. Cambridge, Mass.: MIT Press, 1963.

[71] C. Kramer, H. Gerhardt, and B. Regenscheit, "Wind tunnels for industrial aerodynamics," Journal of Wind Engineering and Industrial Aerodynamics, vol. 16, pp. 225-264, 1984.

[72] M. Rennie, "Effect of jet length on pressure fluctuations in 3/4 open-jet wind tunnels," Research Gate Publication, vol. January, pp. 1-14, 2000.

[73] W. Quinn and J. Militzer, "Experimental and numerical study of a turbulent free square jet," Physics of Fluids, vol. 31, pp. 1017-1025, 1988.

[74] J. V. Ditshuizen and et al., "Acoustic capabilities of the german-dutch wind tunnel dnw," in AIAA 21st Aerospace Sciences Conference, pp. Ref Paper: 830146, January 1983.

[75] G. Manuel and J. Molloy, Effect of Collector Configuration on Test Section Turbulence Levels in an Open-Jet Wind Tunnel. NASA Langley Research Center: NASA Technical Memorandum 4333, 1992.

[76] F. White, Fluid Mechanics. New York: McGraw-Hill, 2011.

[77] R. Blevins, Applied Fluid Dynamics Handbook. New York: Van Nostrand Reinhold Co., 1984.

[78] R. Fox and S. Kline, "Flow regime data and design methods for curved subsonic diffusers," Journal of Basic Engineering, vol. 84, pp. 303-312, 1962.

[79] P. Runstadler and et al., Diffuser Data Book: Diffuser Data Interpretation. Creare Inc.: Technical Note TN-186, 1975. 
[80] G. Johl, M. Passmore, and P. Render, "Design and performance of thin, circular arc, wind-tunnel turning vanes," Royal Aeronautical Society, vol. 111, pp. 115118, 2007.

[81] T. Gelder, R. Moore, J. Sanz, and E. McFarland, Wind Tunnel Turning Vanes of Modern Design. NASA Lewis Research Center: NASA Technical Memorandum $87 / 46,1986$.

[82] J. Rozell, "Duct turning vanes in 90 degree elbows," ASHRAE Technical Document No.230\%, vol. 80, pp. 53-81, 1974.

[83] S. Tavoularis, Measurement in Fluid Mechanics. New York: Cambridge University Press, 2005.

[84] S. Pope, Turbulent Flows. New York: Cambridge University Press, 2000.

[85] Omega Engineering, Stamford, CT, PX140 Series Pressure Transducers Instruction Sheet, m0258/1105 ed., 2010.

[86] Bruel \& Kjaer, Naerum, Denmark, Technical Documentation - Microphone Handbook, vol. 1: theory ed., 1996.

[87] J. Robertson, "Prediction of in-flight fluctuating pressure environments including protuberance induced flow," Wyle Laboratories, vol. Technical Report WR 71-10, pp. 1-94, 1971.

[88] M. Lowson, "Prediction of boundary layer pressure fluctuations," Air Force Flight Dynamics Laboratory, Wright-Patterson Air Force Base, Ohio, vol. Technical Report AFFDL-TR-67-167, pp. 1-40, 1968.

[89] B. Efimtsov, "Similarity criteria for the spectra of wall pressure fluctuations in a turbulent boundary layer," Soviet Physics-Acoustics, vol. 30, pp. 33-35, 1984. 
[90] A. Laganelli and H. Wolfe, "Prediction of fluctuating pressure in attached and separated turbulent boundary-layer flow," Journal of Aircraft, vol. 30, pp. 962970, 1993.

[91] D. Chase, "Modeling the wavevector-frequency spectrum of turbulent boundary layer wall pressure," Journal of Sound and Vibration, vol. 70, pp. 29-67, 1980.

[92] M. Howe, "On the structure of the turbulent boundary-layer wall pressure spectrum in the vicinity of the acoustic wavenumber," Proceedings of the Royal Society of London, vol. A, 412, pp. 389-401, 1987.

[93] R. Rackl and A. Weston, "Modeling of turbulent boundary layer surface pressure fluctuation auto and cross spectra - verification and adjustments based on tu1441 data," NASA Langley Research Center, vol. Contractor Report CR-2005213938, pp. 1-88, 2005.

[94] R. Simpson, M. Ghodbane, and B. McGrath, "Surface pressure fluctuations in a separating turbulent boundary layer," Journal of Fluid Mechanics, vol. 177, pp. 167-186, 1987.

[95] G. Corcos, "Resolution of pressure in turbulence," Journal of the Acoustical Society of America, vol. 35, pp. 192-199, 1963.

[96] S. Jaeger, W. Horne, and C. Allen, "Effect of surface treatment on array microphone self-noise," American Institute of Aeronautics and Astronautics, vol. AIAA-2000-1937, pp. 1-10, 2000.

[97] ANSYS Inc., ANSYS CFX-Solver Modeling Guide, release 17.0 ed., 2017.

[98] ANSYS Inc., ANSYS CFX-Solver Theory Guide, release 17.0 ed., 2017. 


\section{Appendix A}

\section{Description of Computational \\ Environment Used for Component Design \\ Validation}

This appendix contains more detailed information regarding the CFD analyses used to produce the component design validation results discussed in Chapter 2. First, the solver is described along with the hardware and general parameters selected for use in the simulations. Next a discussion regarding the meshing strategies used is provided with summaries of the parameters used to assess mesh quality. Finally, the metrics used to determine solution and proper mesh size convergence are outlined.

\section{A.1 Computer Hardware and CFD Solver}

The numerical simulations were conducted using the ANSYS CFX 15.0 software package. This software uses a finite volume numerical method to solve the Reynolds Averaged Navier-Stokes (RANS) equations iteratively for a 3-D discretized domain at the centre nodes of each constructed control volume. The solution variables stored at

each node are then interpolated for at the faces of each mesh element surrounding the 
corresponding node. The computer hardware used contains an Intel Core i7-4700HQ processing unit with 32 GB of virtual memory (RAM). This hardware allowed the use of local parallel processing schemes to partition the problem for faster computations, as well as the ability to implement mesh sizes up to 10 million nodes.

In general, all simulations were conducted with similar base solver parameters and boundary conditions. This included the implementation of a steady-state analysis with the fluid domain modeled as a compressible, ideal gas. All solid walls surrounding the fluid domain were modeled using the no-slip condition with no heat transfer to and from the wall-to-fluid interface (adiabatic walls). Inlet and outlet boundary conditions were selected with a uniform inlet velocity and an area averaged static pressure condition respectively (the values of which were determined using the corresponding expected flow conditions at each respective point). Turbulence intensity levels at the inlet were specified as medium (5\%) for general components and low (1\%) in areas after flow conditioners are placed (nozzle and jet orifice boundaries). The total energy heat transfer model was chosen with the viscous work term enabled to properly model enthalpy transport within the fluid while including for kinetic energy effects (high speed compressible flows) [97, 98]. The Shear-Stress Transport (SST) turbulence model was employed as opposed to other models such as the k-epsilon $(k$ $\epsilon)$ or k-omega $(k-\omega)$. The SST model is recommended by CFX [97] for more accurate prediction of flow separation along walls since it applies a blend of using low Reynolds number formulations near the wall rather than a $(k-\epsilon)$ wall function formulation which is not valid for boundary layer refined meshes with a first cell $y^{+} \leq 11$. This model thus requires a refined mesh in the boundary layer with enough points to accurately model the viscous sublayer, therefore, the mesh must contain a first cell $y^{+} \leq 1-2$ [97, 98]. Other selected solver parameters include using the double precision solver environment, a high resolution advection scheme, and first order turbulence numerics. 


\section{A.2 Domain Mesh Strategy and Quality Metrics}

The domain meshes for all components with relatively simple geometries (nozzle, diffuser, etc.) were constructed in ANSYS ICEM CFD 15.0 due to the ability to have total manual control over the mesh blocking topology and boundary layer refinement regions. All ICEM meshes are originally constructed using structured hexahedral elements, which are then technically converted to an unstructured domain for input into the CFX solver (program requirement). Other components with more intricate domain geometries (transition channel, jet collector, etc.) were meshed using the ANSYS CFX-Mesh 15.0 automatic discretization software. This software produces a computer generated mesh of the domain based on the selection of overall mesh size and refinement parameters. This software is advantageous for use with complicated geometries that would require significant effort to produce in ICEM at the expense of less control over the mesh topology and quality. These meshes can also only be generated with tetrahedral elements when a wall inflation method is used to refine the boundary layer. All meshes were generated with a refined inflation region at the wall boundaries to accurately capture the boundary layer and possible regions of flow separation. The goal of the refinement was to achieve a first cell $y^{+} \leq 1-2$ and to obtain a boundary layer cell inflation ratio $\leq 1.1$.

Figure A.1 contains a general comparison of the component meshes generated in ICEM and CFX-Mesh programs. The difference in the element geometry can be seen, along with the broad strategy used to refine the mesh in the boundary layer regions. A body of influence method was also employed to refine the mesh in specific areas of required high solution accuracy (test section jet core and shear layer).

Aside from the aforementioned boundary layer mesh refinement criteria, both software packages can assess the overall quality of a given mesh using the following 


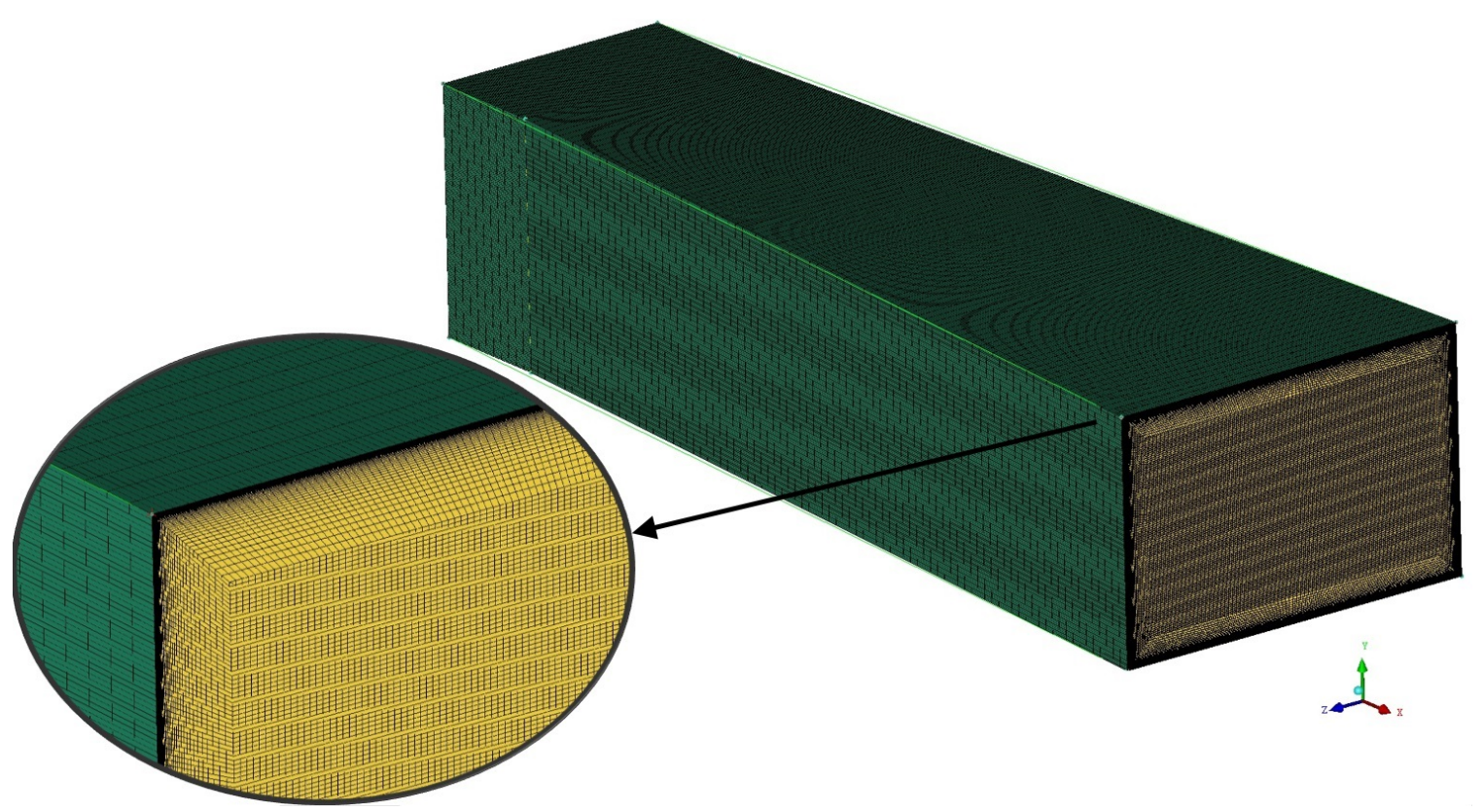

(a) ICEM CFD software generated mesh (vertical exhaust diffuser example)

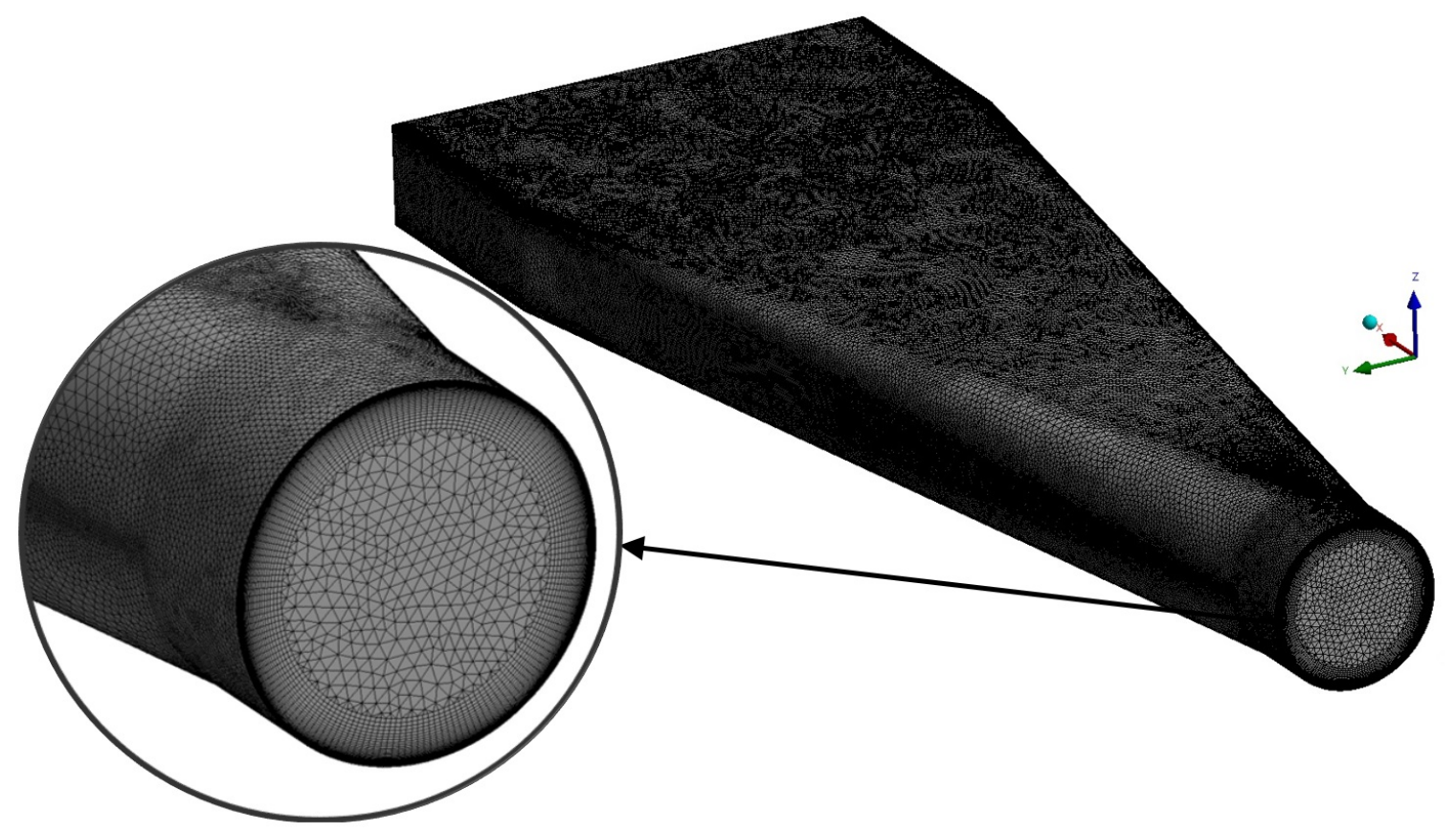

(b) CFX-Mesh software generated mesh (transition channel example)

Figure A.1: General comparison of component domain meshing strategies. 
calculated metrics: the determinant, minimum internal angle and volume expansion factor [97]. CFX recommends the normalized determinant of the Jacobian of any mesh element be greater than 0.3. The minimum acceptable internal face angle of any mesh element is $18^{\circ}$. Finally, the ratio of volumes between any successive mesh elements must be less than 20. It is important to note that the mesh element aspect ratio is not a particular issue when a double precision simulation environment is used [97]. General quality metrics achieved for the component meshes include: determinant around 0.9 (ICEM Meshes) and 0.7 (CFX Meshes); minimum angle greater than $25^{\circ}$; and an expansion ratio less than 3 .

\section{A.3 Solution Convergence and Sensitivity Studies}

CFD solutions are generally assessed for convergence by obtaining a certain lower threshold of the Root-Mean-Square (RMS) residuals or imbalances of the conservative control volume equations being solved through each solution iteration. CFX sets a default RMS residual target of $10^{-4}$ to achieve 'loose' solution convergence for the mass continuity, momentum, and energy equations [97]. Solutions with higher RMS residual values may only be sufficient enough for a qualitative assessment of the flow field. RMS values lower than $10^{-5}$ generally indicate a solution with proper convergence. More sensitive problems requiring high accuracy in calculated flow parameters will require tighter convergence.

Figure A.2 provides an example of the solution convergence behavior of the conducted simulations. The example provided corresponds to the nozzle channel simulations where excellent convergence was obtained. In general, all of the components with ICEM generated meshes and relatively simple geometries achieved RMS residuals below $10^{-5}$ with solution imbalances within $\pm 0.1 \%$. The maximum RMS residuals 
obtained was just under $10^{-4}$ with imbalance levels within $\pm 1 \%$. This corresponded to the test section and jet collector CFD simulations which contain more complicated geometric features and lower overall mesh quality.

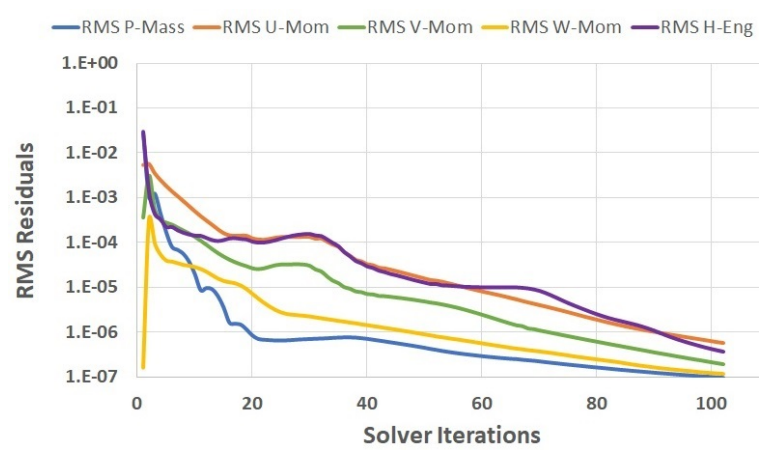

(a) Solution RMS residual convergence plots

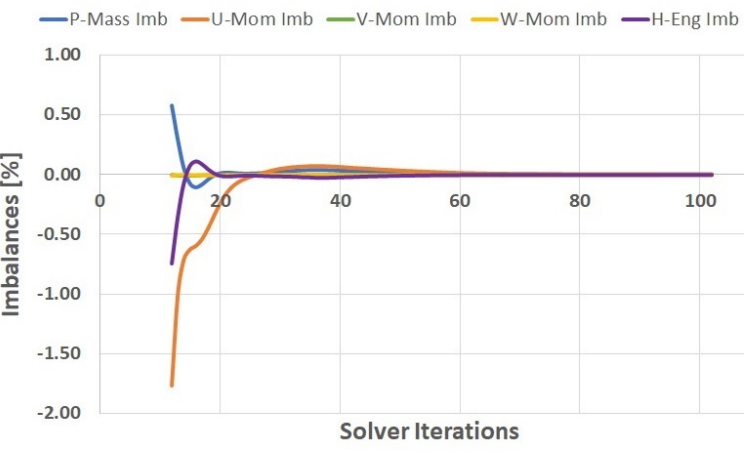

(b) Solution equation imbalances convergence plots

Figure A.2: General simulation solution convergence behavior (nozzle channel example).

Accurate solutions must also be assessed in terms of acquiring convergence in calculated flow parameters of interest. For this study, convergence on the mass flow averaged outlet Mach number, mass flow averaged total pressure difference, and area averaged $y^{+}$values along the walls was ensured for each simulation. Figure A.3 contains example convergence behavior for these flow parameters. Brief sensitivity studies were also conducted with respect to changes in mesh size (number of nodes) on the solution parameters. All of the conducted simulations used meshes with a high number of element nodes, therefore, these were repeated using meshes with a smaller amount of elements (roughly $1 / 3$ reduction) to verify that the solution flow parameters did not vary by more than $1 \%$. It was generally found that the meshes used were over-refined and produced insensitive solution outputs with respect to minor reductions in mesh refinement. 


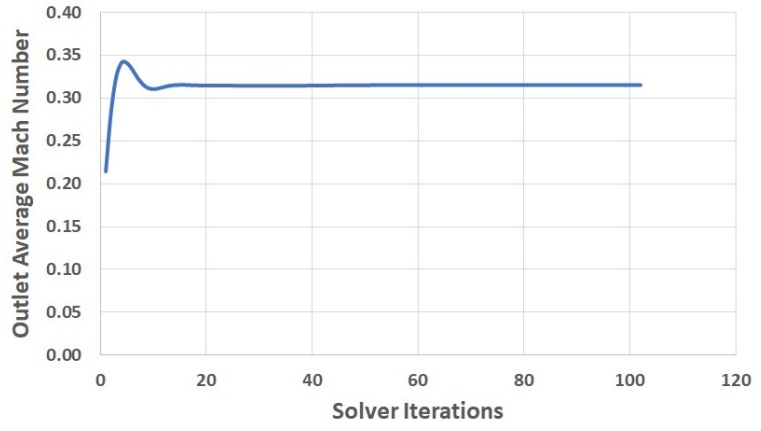

(a) Outlet Mach number convergence

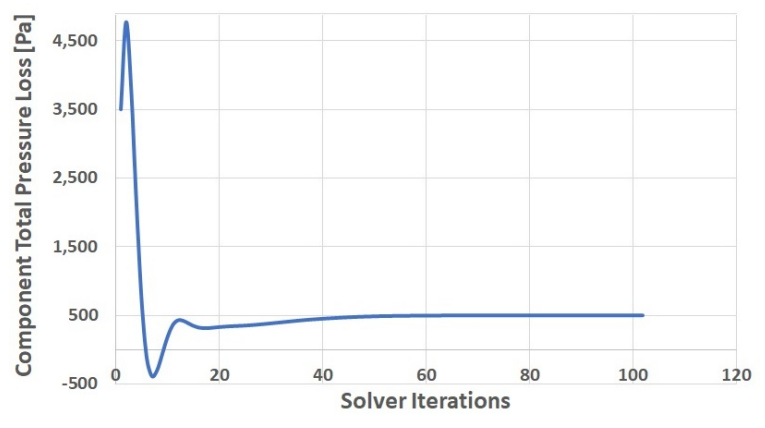

(b) Total component pressure loss convergence

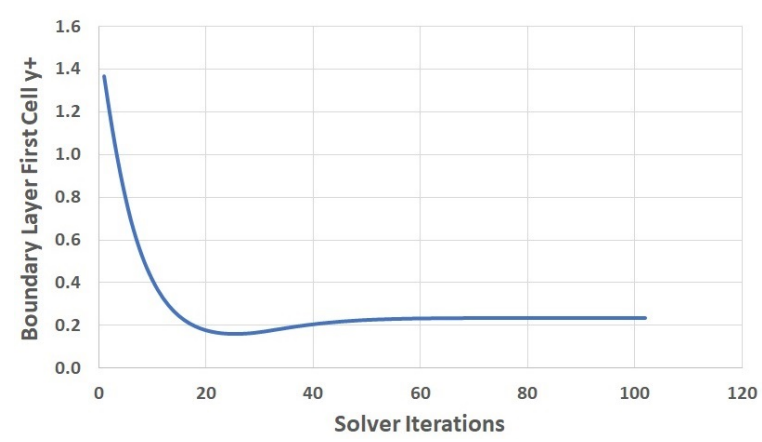

(c) Boundary layer first cell $y^{+}$convergence

Figure A.3: Typical flow parameter convergence behavior (nozzle channel example). 